C. 2

\title{
Plant Community Classification of the Pakowki Sandhills and Sand Plains
}

Prepared For:

Resource Data Branch

Alberta Sustainable Resource Development

Edmonton, Alberta

Prepared By:

Geowest Environmental Consultants Ltd.

January 2003

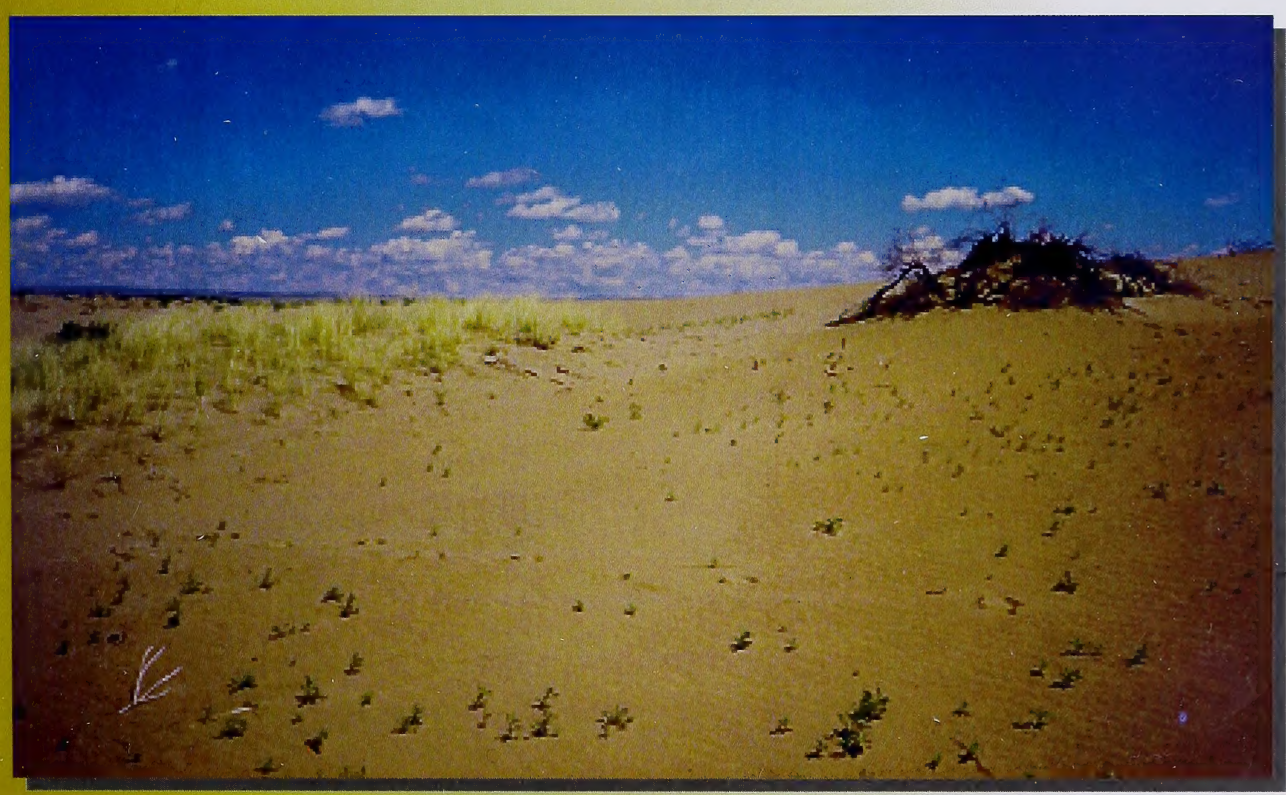


Digitized by the Internet Archive in 2015

https://archive.org/details/plantcommunitycl00coen 


\title{
Plant Community Classification of the Pakowki Sandhills and Sand Plains
}

\author{
Prepared for: \\ Resource Data Branch \\ Alberta Sustainable Resource Development \\ Edmonton, Alberta \\ Prepared by: \\ Valerie Coenen \\ Jerry Bentz
}

Geowest Environmental Consultants Ltd.

Suite 203, 4208 - 97 Street

Edmonton, Alberta

Canada

T6E 5Z9

January, 2003 



\section{Executive Summary}

The Resource Data Division of Alberta Sustainable Resource Development contracted Geowest Environmental Consultants Ltd. to produce a classification of sand dune and sand plain plant community types within the Grassland Natural Region of Alberta. This initiative is in support of the Alberta Natural Heritage Information Centre (ANHIC). ANHIC collects, evaluates and makes available data on elements of natural biodiversity in Alberta, including flora, fauna and native plant communities. ANHIC develops tracking lists of elements that are considered of high priority because they are considered rare or special in some way. ANHIC's long-term goal is to develop a list of plant community types that occur throughout the province and to attempt to identify community types that require conservation initiatives.

The primary objective of this project was to develop a plant community classification of sand dune and sand plain plant communities of the Grassland Natural Region based on field survey, correlations with other surveys and any available previously-collected data. Furthermore, each identified community type was evaluated and assigned a preliminary provincial rank, based on its rarity/endemism or threats to its condition. This was accomplished by developing a sampling protocol and subsequently collecting field data on plant communities of the sand dune and sand plain landscapes of the Pakowki Sandhills. Furthermore, a comparison of defined plant community types to similar types described in previously conducted field surveys in similar landscapes in Alberta and adjacent provinces and states was completed. A similarity rating based on a scale provided by Corns (1983) was also provided.

This classification will provide a better understanding of plant community biodiversity in Alberta and will contribute to the development of a Canadian National Vegetation Classification (CNVC), the Canadian component of the International Classification of Ecological Communities (ICEC). The ICEC system has been adopted by the United States and it is a national standard for vegetation classification known as the U.S. National Vegetation Classification (USNVC).

An exhaustive literature search was completed, to locate references relating to sand dune and sand plain plant communities in Alberta. Literature related to other jurisdictions was also obtained, primarily for Saskatchewan, Montana, Idaho, Wyoming, North and South Dakota and Nebraska.

Field sampling occurred between July 26th and 30th, 2002 following a review of the sampling strategy with Alberta Sustainable Resource Development staff. In total 40 sampling plots were established, distributed throughout a range of community types and topographic positions. Survey sites were selected based on an initial review of the survey area, using aerial photographs and vegetation trends observed in the field.

Cluster and ordination analyses were performed on the field data resulting in 17 community types based on the hierarchical guidelines documented in the International Classification of Ecological Communities: Terrestrial Vegetation of the United States: Volume 1 - The National Classification System: Development, Status and Application (Grossman et al. 1998). 
Each community type identified from the analysis of the plot data was compared against floristically similar community types described for Alberta and other jurisdictions. A summary of the findings are provided as correlation tables, which compare the Pakowki Sandhills community types against community types described in literature based on Alberta and other jurisdictions, respectively. A similarity rating between the community types, based on Corns (1983) and recently applied by Strong (2002), was also provided in the tables. A discussion of the community types and associated literature was also provided.

All proposed community types were assigned a preliminary provincial ranking. Knowledge gaps were identified and strategies to address these gaps were provided. The information in this report can be used to update the community-tracking list by including new community types. Finally, this report can also be used to decide which community types require further studies and to prioritize these studies. 


\section{Acknowledgements}

We wish to thank Lorna Allen and Ksenija Vujnovic (Alberta Natural Heritage Information Centre - Alberta Community Development) and Keith Ainsley (Resource Data Division - Alberta Sustainable Resource Development) for assistance throughout this project. The services of Kathy Tannas are also greatly appreciated for identification and verification of plant species. We are also grateful to Erin Anderson for assistance with fieldwork, as well as Dennis O'Leary and Terry Lang (Geowest Environmental Consultants Ltd.) who contributed to the successful completion of this project. 


\section{Table of Contents}

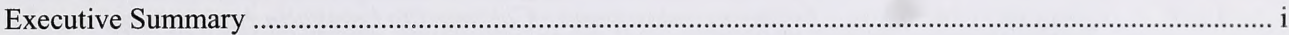

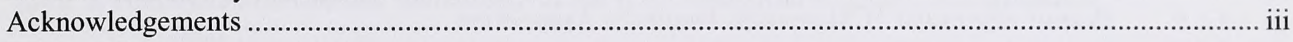

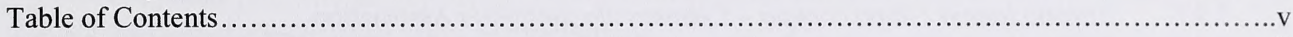

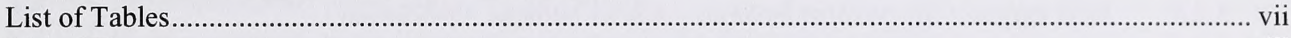

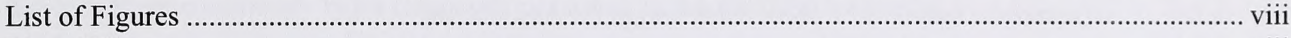

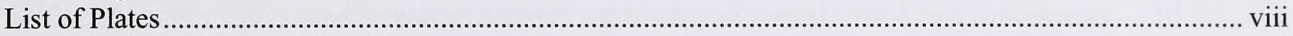

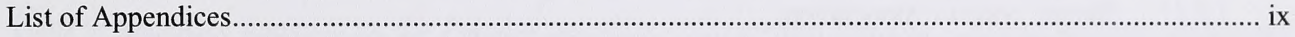

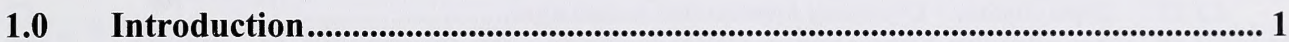

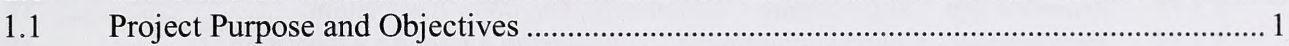

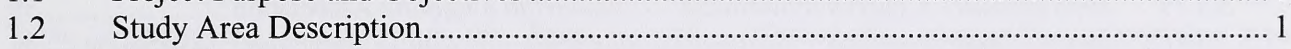

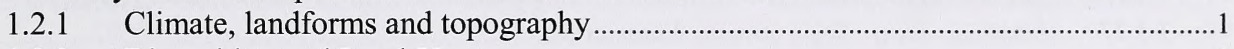

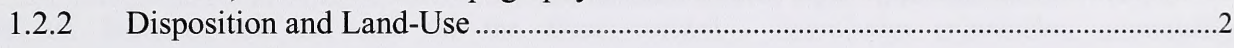

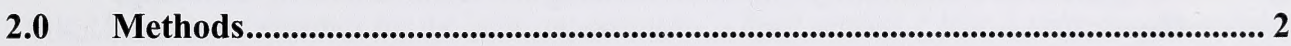

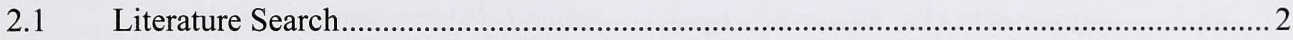

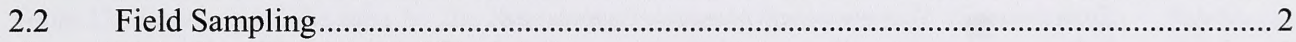

2.3 Plant Identification Verification ................................................................................. 3

$2.4 \quad$ Vegetation Data Analysis and Classification........................................................... 3

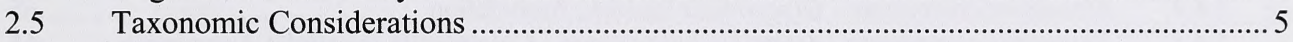

2.6 Cross-referencing of Proposed Community Types to Literature ..................................... 5

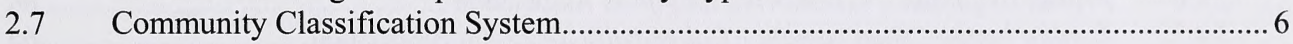

2.8 Evaluation and Assignment of Preliminary Provincial Ranking .................................. 6

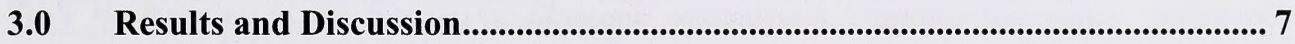

3.1 Vegetation Data Analysis and Classification............................................................. 7

3.2 Preliminary Classification of Community Types ....................................................... 11

3.2.1 Description of Community Types .................................................................................12

3.2.1.1 Populus deltoides / Glycyrrhiza lepidota - Juncus balticus Association ...........................13

3.2.1.2 Rosa woodsii / Sporobolus cryptandrus Association......................................................15

3.2.1.3 Salix amygdaloides - Rosa woodsii / Juncus balticus - Sporobolus cryptandrus

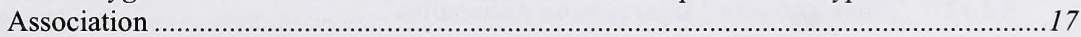

3.2.1.4 Elaeagnus commutata / Glycyrrhiza lepidota Association .........................................19

3.2.1.5 Elaeagnus commutata / Artemisia ludoviciana - Calamovilfa longifolia Association ......21

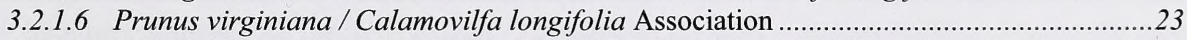

3.2.1.7 Eurotia lanata / Stipa comata - Calamovilfa longifolia Association.................................25

3.2.1.8 Salix exigua / Glycyrrhiza lepidota - Juncus balticus Association ..................................27

3.2.1.9 Glycyrrhiza lepidota - Calamovilfa longifolia Association .............................................29

3.2.1.10 Glycyrrhiza lepidota-Artemisia spp. - Stipa comata Association ................................. 31

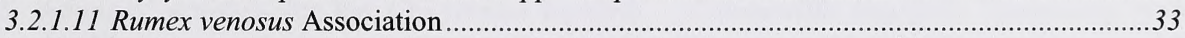

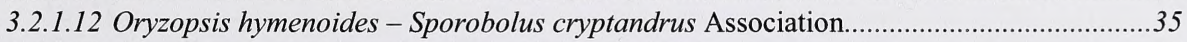

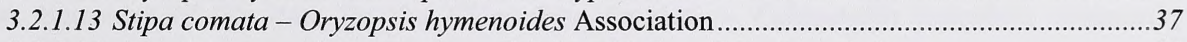

3.2.1.14 Artemisia spp. - Stipa comata - Calamovilfa longifolia Association ...................................

3.2.1.15 Stipa comata - Cyperus schweinitzii - Calamovilfa longifolia Association.......................41

3.2.1.16 Sporobolus cryptandrus - Calamovilfa longifolia-Oryzopsis hymenoides Association..43

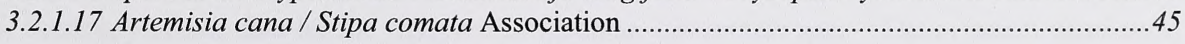

3.3 Cross-Referencing of Proposed Community Types with Literature.............................. 47

3.3.1 Populus deltoides / Glycyrrhiza lepidota - Juncus balticus Association .................................47 
3.3.2 Rosa woodsii / Sporobolus cryptandrus Association.

3.3.3 Salix amygdaloides - Rosa woodsii / Juncus balticus - Sporobolus cryptandrus Association.............................................................50

3.3.4 Elaeagnus commutata / Glycyrrhiza lepidota Association.................................................50

3.3.5 Elaeagnus commutata / Artemisia ludoviciana-Calamovilfa longifolia Association ..........51

3.3.6 Prunus virginiana / Calamovilfa longifolia Association ....................................................53

3.3.7 Eurotia lanata / Stipa comata - Calamovilfa longifolia Association ....................................54

3.3.8 Salix exigua / Glycyrrhiza lepidota - Juncus balticus Association......................................54

3.3.9 Glycyrrhiza lepidota - Calamovilfa longifolia Association................................................56

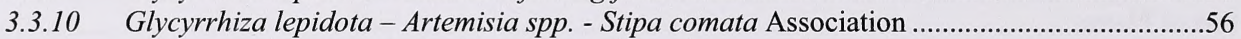

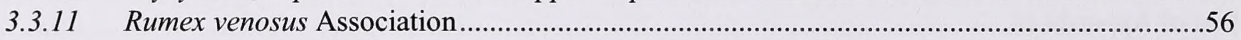

3.3.12 Oryzopsis hymenoides - Sporobolus cryptandrus Association ........................................57

3.3.13 Stipa comata-Oryzopsis hymenoides Association .........................................................57

3.3.14 Artemisia spp. - Stipa comata - Calamovilfa longifolia Association ....................................59

3.3.15 Stipa comata - Cyperus schweinitzii - Calamovilfa longifolia Association ........................59

3.3.16 Sporobolus cryptandrus - Calamovilfa longifolia-Oryzopsis hymenoides Association .....60

3.3.17 Artemisia cana / Stipa comata Association ...............................................................62

3.4 Assignment of a Preliminary Provincial Ranking and Identification of Knowledge

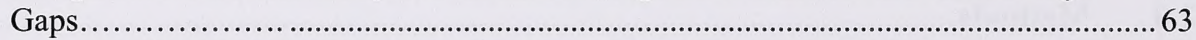

3.4.1 Populus deltoides / Glycyrrhiza lepidota - Juncus balticus Association ............................63

3.4.2 Rosa woodsii / Sporobolus cryptandrus Association....................................................64

3.4.3 Salix amygdaloides - Rosa woodsii /Juncus balticus - Sporobolus cryptandrus

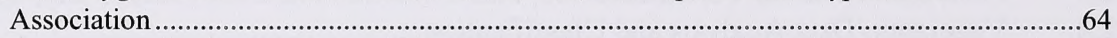

3.4.4 Elaeagnus commutata / Glycyrrhiza lepidota Association...............................................65

3.4.5 Elaeagnus commutata / Artemisia ludoviciana - Calamovilfa longifolia Association ...........65

3.4.6 Prunus virginiana / Calamovilfa longifolia Association ....................................................66

3.4.7 Eurotia lanata / Stipa comata - Calamovilfa longifolia Association...................................66

3.4.8 Salix exigua / Glycyrrhiza lepidota - Juncus balticus Association......................................66

3.4.9 Glycyrrhiza lepidota - Calamovilfa longifolia Association..............................................67

3.4.10 Glycyrrhiza lepidota-Artemisia spp. - Stipa comata Association ..................................67

3.4.11 Rumex venosus Association ...................................................................................67

3.4.12 Oryzopsis hymenoides - Sporobolus cryptandrus Association .........................................68

3.4.13 Stipa comata-Oryzopsis hymenoides Association........................................................68

3.4.14 Artemisia spp. - Stipa comata - Calamovilfa longifolia Association...................................69

3.4.15 Stipa comata - Cyperus schweinitzii-Calamovilfa longifolia Association ........................69

3.4.16 Sporobolus cryptandrus - Calamovilfa longifolia-Oryzopsis hymenoides Association .....70

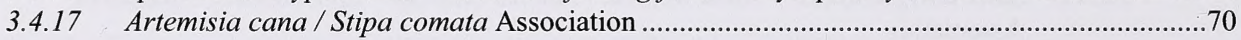

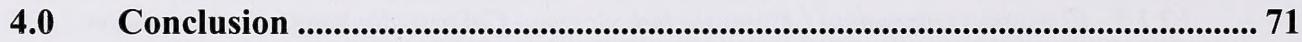

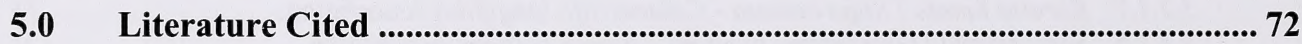




\section{List of Tables}

Table 1. Summary of climatic data for the Dry Mixedgrass Natural Subregion. . . . . . . . . . . . . . . . . . . . . . ...2

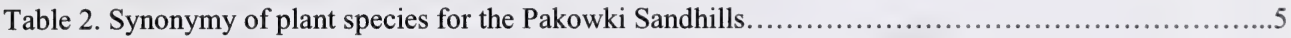

Table 3. Hierarchical levels and definitions for the ICEC terrestrial vegetation classification

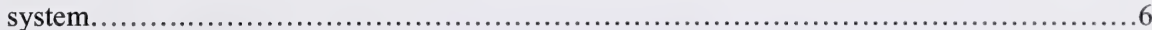

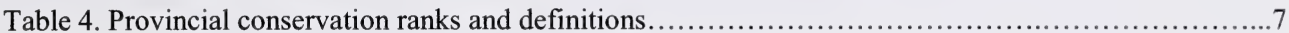

Table 5. Plant community types (associations) found in the Pakowki Sandhills........................11

Table 6. Numeric codes used to define different vegetation structural strata.........................12

Table 7. Summary statistics for the Populus deltoides / Glycyrrhiza lepidota - Juncus balticus community

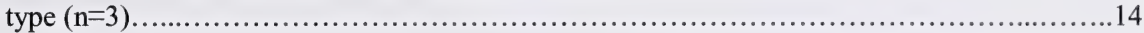

Table 8. Summary of site data for the Populus deltoides / Glycyrrhiza lepidota - Juncus balticus

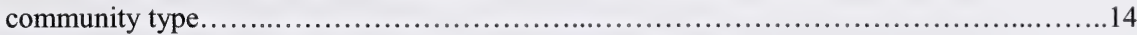

Table 9. Summary statistics for the Rosa woodsii / Sporobolus cryptandrus community type $(n=4)$

Table 10. Summary of site data for the Rosa woodsii / Sporobolus cryptandrus community type................................................................................. 16

Table 11. Summary statistics for the Salix amygdaloides - Rosa woodsii / Juncus balticus - Sporobolus

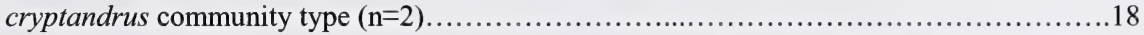

Table 12. Summary of site data for the Salix amygdaloides - Rosa woodsii / Juncus balticus - Sporobolus cryptandrus community type........................................................... 18

Table 13. Summary statistics for the Elaeagnus commutata / Glycyrrhiza lepidota community type $(n=2)$

Table 14. Summary of site data for the Elaeagnus commutata / Glycyrrhiza lepidota community

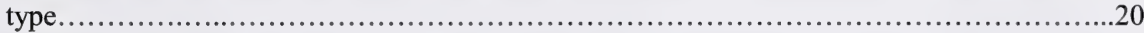

Table 15. Summary statistics for the Elaeagnus commutata / Artemisia ludoviciana - Calamovilfa

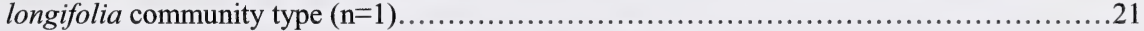

Table 16. Summary of site data for the Elaeagnus commutata / Artemisia ludoviciana - Calamovilfa longifolia community type.............................................................. 22

Table 17. Summary statistics for the Prunus virginiana / Calamovilfa longifolia community type

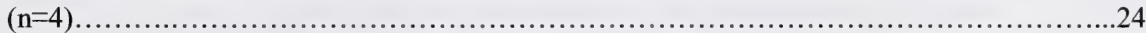

Table 18. Summary of site data for the Prunus virginiana / Calamovilfa longifolia community

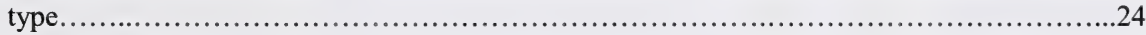

Table 19. Summary statistics for the Eurotia lanata / Stipa comata - Calamovilfa longifolia community

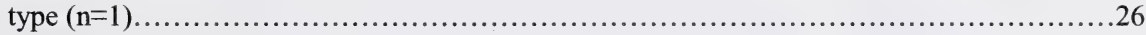

Table 20. Summary of site data for the Eurotia lanata / Stipa comata-Calamovilfa longifolia community

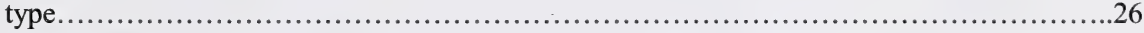

Table 21. Summary statistics for the Salix exigua / Glycyrrhiza lepidota - Juncus balticus community type

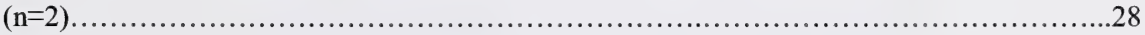

Table 22. Summary of site data for the Salix exigua / Glycyrrhiza lepidota - Juncus balticus community type.............................................................................28

Table 23. Summary statistics for the Glycyrrhiza lepidota - Calamovilfa longifolia community type $(\mathrm{n}=1)$.

Table 24. Summary of site data for the Glycyrrhiza lepidota - Calamovilfa longifolia community type.................................................................................

Table 25. Summary statistics for the Glycyrrhiza lepidota - Artemisia spp. - Stipa comata community type $(\mathrm{n}=2)$.......

Table 26. Summary of site data for the Glycyrrhiza lepidota - Artemisia spp. - Stipa comata community type...

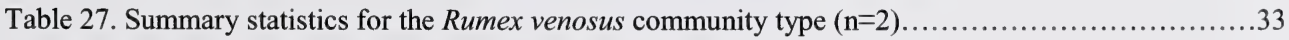

Table 28. Summary of site data for the Rumex venosus community type 
Table 29. Summary statistics for the Oryzopsis hymenoides - Sporobolus cryptandrus community type

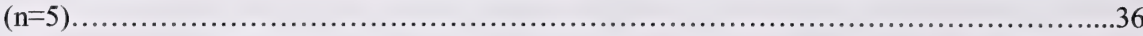

Table 30. Summary of site data for the Oryzopsis hymenoides - Sporobolus cryptandrus community

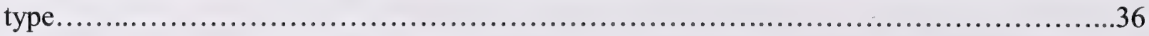

Table 31. Summary statistics for the Stipa comata - Oryzopsis hymenoides community type

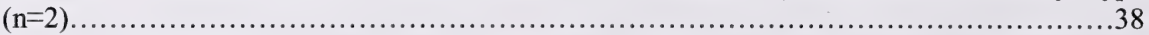

Table 32. Summary of site data for the Stipa comata - Oryzopsis hymenoides community type.

Table 33. Summary statistics for the Artemisia spp. - Stipa comata - Calamovilfa longifolia community type $(n=3)$.

Table 34. Summary of site data for the Artemisia spp. - Stipa comata - Calamovilfa longifolia community

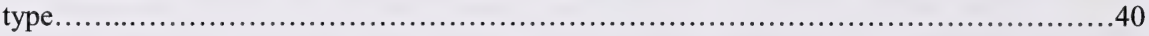

Table 35. Summary statistics for the Stipa comata - Cyperus schweinitzii - Calamovilfa longifolia

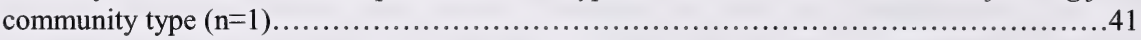

Table 36. Summary of site data for the Stipa comata - Cyperus schweinitzii - Calamovilfa longifolia community type.............................................................. 42

Table 37. Summary statistics for the Sporobolus cryptandrus - Calamovilfa longifolia - Oryzopsis

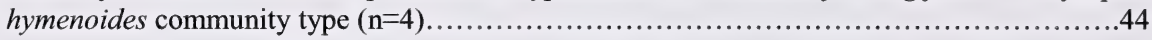

Table 38. Summary of site data for the Sporobolus cryptandrus - Calamovilfa longifolia - Oryzopsis hymenoides community type.....................................................44

Table 39. Summary statistics for the Artemisia cana / Stipa comata community type

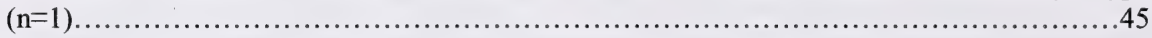

Table 40. Summary of site data for the Artemisia cana / Stipa comata community

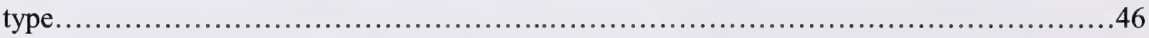

\section{List of Figures}

Figure 1. Cluster analysis of 40 plots collected July 2002.

Figure 2. Ordination diagram based on Detrended Correspondence Analysis (DCA) of 40 plots collected in July 2002 ......

Figure 3. Biplot based on Detrended Correspondence Analysis (DCA) of sample plots (40) and plant species (65) collected at the Pakowki Sandhills in July 2002.

\section{List of Plates}

Plate 1. Populus deltoides / Glycyrrhiza lepidota-Juncus balticus community type (Western cottonwood /

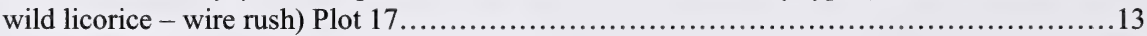

Plate 2. Rosa woodsii / Sporobolus cryptandrus community type (Common wild rose / sand dropseed) Plot

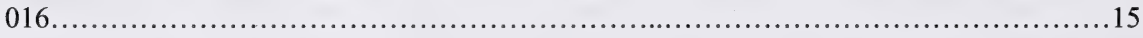

Plate 3. Salix amygdaloides - Rosa woodsii / Juncus balticus - Sporobolus cryptandrus (Peach-leaf willow

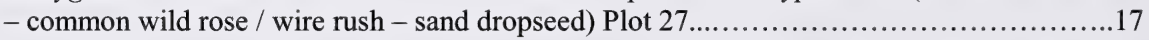

Plate 4. Elaeagnus commutata / Glycyrrhiza lepidota community type (Silverberry / wild licorice) Plot

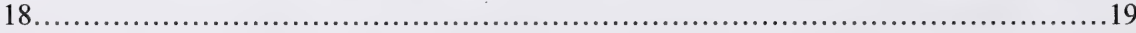

Plate 5. Elaeagnus commutata / Artemisia ludoviciana - Calamovilfa longifolia (Silverberry / prairie

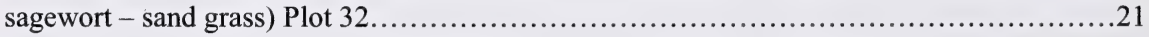

Plate 6. Prunus virginiana / Calamovilfa longifolia community type (Chokecherry / sand grass) Plot

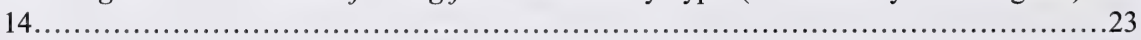

Plate 7. Eurotia lanata / Stipa comata - Calamovilfa longifolia community type (Winterfat / needle and

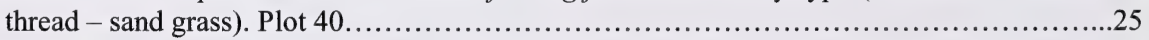

Plate 8. Salix exigua / Glycyrrhiza lepidota - Juncus balticus community type (Sandbar willow / wild

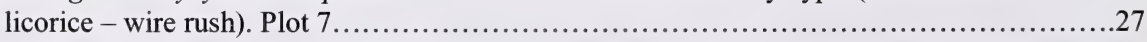

Plate 9. Glycyrrhiza lepidota - Calamovilfa longifolia community type (Wild licorice - sand grass) Plot

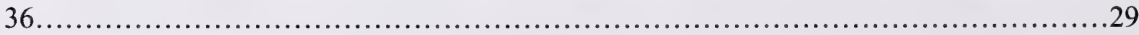


Plate 10. Glycyrrhiza lepidota - Artemisia spp. - Stipa comata community type (Wild licorice - sage -

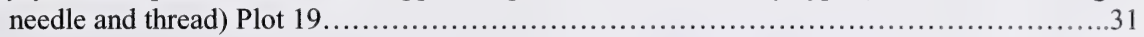

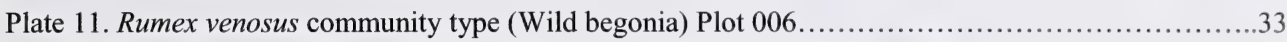

Plate 12. Oryzopsis hymenoides - Sporobolus cryptandrus community type (Indian rice grass - sand

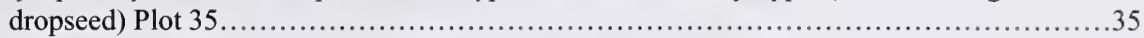

Plate 13. Stipa comata - Oryzopsis hymenoides community type (Needle and thread - Indian rice grass) Plot 8 ......

Plate 14. Artemisia spp. - Stipa comata - Calamovilfa longifolia community type (Sage - needle and

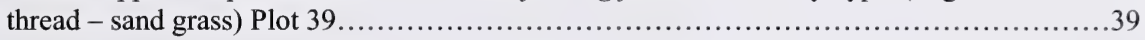

Plate 15. Stipa comata - Cyperus schweinitzii - Calamovilfa longifolia community type (Needle and thread

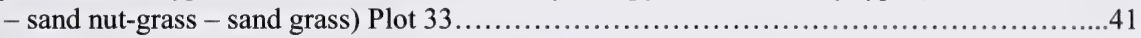

Plate 16. Sporobolus cryptandrus - Calamovilfa longifolia - Oryzopsis hymenoides (Sand dropseed - sand

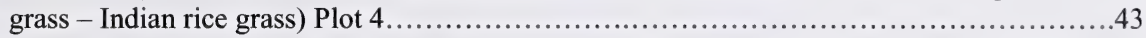

Plate 17. Artemisia cana / Stipa comata community type (Silver sagebrush / needle and thread). Plot 24

\section{List of Appendices}

Appendix 1. Glossary of Scientific and Common Plant Species Names

Appendix 2. PC-ORD output from Detrended Correspondence Analysis of Plot Data

Appendix 3. Species Code Descriptions

Appendix 4. Correlation table with literature from within Alberta

Appendix 5. Correlation table with literature from outside Alberta 


\subsection{Introduction}

\subsection{Project Purpose and Objectives}

The Resource Data Division of Alberta Sustainable Resource Development contracted Geowest Environmental Consultants Ltd. to produce a classification of sand dune and sand plain plant community types within the Grassland Natural Region of Alberta. This initiative is in support of the Alberta Natural Heritage Information Centre (ANHIC). ANHIC collects, evaluates and makes available data on elements of natural biodiversity in Alberta, including flora, fauna and native plant communities. ANHIC develops tracking lists of elements that are considered of high priority because they are rare or special in some way. ANHIC's long-term goal is to develop a list of plant community types that occur throughout the province and to attempt to identify community types that require conservation initiatives.

The primary objective of this project was to develop a plant community classification of sand dune and sand plain plant communities of the Grassland Natural Region based on field survey, correlations with other surveys and any available previously-collected data. Furthermore, each identified community type was evaluated and assigned a preliminary provincial rank, based on its rarity/endemism or threats to its condition. This was accomplished by developing a sampling protocol and subsequently collecting field data on plant communities of the sand dune and sand plain landscapes of the Pakowki Sandhills. Furthermore, a comparison of defined plant community types to similar types described in previously conducted field surveys in similar landscapes in Alberta and adjacent provinces and states was completed. A similarity rating based on a scale provided by Corns (1983) was also provided.

This classification will provide a better understanding of plant community biodiversity in Alberta and will contribute to the development of a Canadian National Vegetation Classification (CNVC), the Canadian component of the International Classification of Ecological Communities (ICEC). The ICEC system has been adopted by the United States and it is a national standard for vegetation classification known as the U.S. National Vegetation Classification (USNVC).

\subsection{Study Area Description}

\subsubsection{Climate, landforms and topography}

The Pakowki Sandhills study area (Pakowki Sandhills) is located in the Dry Mixedgrass Natural Subregion (Achuff 1994) and is representative of sand dune and sand plain ecosystems of this subregion. The climate of the Dry Mixedgrass Subregion is the warmest and driest in all of Alberta (Table 1). It has a characteristic continental climate with cold winters, warm summers and relatively low precipitation. Total annual precipitation is generally $260-280 \mathrm{~mm}$, the lowest summer precipitation values compared to every other subregion in the province (Alberta Environmental Protection 1997). Winds are often quite high and chinooks are regular events during the winter months. 
Table 1. Summary of climatic data for the Dry Mixedgrass Natural Subregion.

\begin{tabular}{|l|l|}
\hline Mean Annual Temperature & $4{ }^{\circ} \mathrm{C}$ \\
\hline Mean Summer Temperature & $16^{\circ} \mathrm{C}$ \\
\hline Mean Winter Temperature & $-7{ }^{\circ} \mathrm{C}$ \\
\hline Total Annual Precipitation & $260-280 \mathrm{~mm}$ \\
\hline
\end{tabular}

The topography of the Pakowki Sandhills is gently rolling with relatively shallow slopes. Dune fields are located in both the northern and southern blocks, comprising approximately $50 \%$ of the northern block and $30 \%$ of the southern block. Both parabolic and longitudinal dunes with blowouts are present in the area (Shetsen 1987). Dunes consist of fine to medium grained sand and silt and reach depths of up to 7 metres. Hummocky stagnation till and ridged end moraine are also prevalent in the study area, with localized fluvial deposits along Irrigation and Canal creeks.

Elevations range from approximately $860 \mathrm{~m}$ at the former Pakowki lakeshore in the southwestern portion of the northern block, to $900 \mathrm{~m}$ in the northeastern corner. Elevations in the southern block range from approximately $860 \mathrm{~m}$ in the east to $915 \mathrm{~m}$ in the southeast.

\subsubsection{Disposition and Land-Use}

The study area consists of a northern block adjacent to and east of the north end of Pakowki Lake and a southern block directly east of the south end of the lake. The Pakowki Sandhills are Crown land in right of the Province of Alberta, administered by Alberta Sustainable Resource Development. Current land uses consist primarily of cattle grazing on native and non-native pasture with some cultivation on adjacent parcels of privately owned land. There are approximately 10 grazing leaseholders within the Pakowki Sandhills.

\subsection{Methods}

\subsection{Literature Search}

An exhaustive literature search was completed to locate references relating to sand dune and sand plain plant communities in Alberta. Literature related to other jurisdictions was also obtained, primarily for Saskatchewan, Montana, Idaho, Wyoming, North and South Dakota and Nebraska. Lorna Allen, of Alberta Community Development, also provided many references.

\section{$2.2 \quad$ Field Sampling}

Field sampling occurred between July 26th and 30th, 2002 following a review of the sampling strategy with Alberta Sustainable Resource Development staff. In total 40 sampling plots were established, distributed throughout a range of community types and topographic positions. All survey sites were accessed on foot. Survey sites were selected based on an initial review of the survey area, using aerial photographs and vegetation trends observed in the field. In most instances, a minimum of three plots were established 
in each observed community type. This was not possible for certain community types, as their distribution was quite limited.

Vegetation and site description forms (RDB 2002-3 and RDB 2002-1 respectively) were completed at each site. The forms were completed using definitions and guidelines from Alberta Environmental Protection (1994). Percent cover of vascular plant species was visually estimated in each plot, using the relevé method. All dominant, codominant and diagnostic species were recorded. Each grass species encountered was collected for expert identification. Furthermore, unknown species were also collected for identification.

Plots were documented with a $35 \mathrm{~mm}$ photograph to characterize the structure and composition of the plant community. The location of each survey site was determined using a Trimble GeoExplorer III unit and the locations were recorded on the site description forms. Furthermore, the location of each plot was marked on the aerial photographs. Each plot was located with a pinprick and circled and annotated on the back of the aerial photograph.

\subsection{Plant Identification Verification}

Each grass species encountered was collected, as were any unknown species. Kathy Tannas, of Eastern Slopes Rangeland Seeds, verified these specimens. The plot forms and digital database were updated, to reflect Ms. Tannas' findings.

\subsection{Vegetation Data Analysis and Classification}

Vegetation data were entered into an Excel spreadsheet, comprising a matrix of field plot versus plant species abundance. The plant species were identified by stratum, as plant community structure was considered to be important in defining community types. The final database contained 40 plots and 65 species columns. The species columns may include duplicate species names, in cases where one species was found in more than one stratum (e.g. Elaeagnus commutata as both a tall and short shrub). However, the different strata were designated by a numeric value following the species code to allow for differentiation. The field data spreadsheet was then imported into PC ORD version 4.20, for classification analysis.

\section{Cluster Analysis}

The vegetation classification incorporated all stages of plant succession and was not restricted to potential or predicted climax associations, following Braun-Blanquet (1965). Cluster analysis was used to allow for a more objective classification of sand dune and sand plain communities based on species composition. An hierarchical, agglomerative clustering technique (Farthest Neighbour Analysis) was used to help identify plant community types. This clustering method progressively combines plots/samples from an individual based on their similarity until all samples are in one group (similarity analysis).

Several clustering options available in PC ORD v.4.20 were explored and the group linkage method with the lowest percent chaining (maximum information) was selected for analysis (Farthest Neighbor). The Bray-Curtis (Sorensen) and Relative Sorensen 
distance measures were investigated and in combination with the Farthest Neighbor group linkage method, provided the lowest percent chaining values. Other distance measures were explored (Euclidean, Relative Euclidean), however, these measures had higher percent chaining values and seemed to introduce confusion into the clustering results.

\section{Detrended Correspondence Analysis}

Detrended correspondence analysis was also investigated to help identify plant community types. Detrended correspondence analysis (DCA) is an indirect gradient analysis/ordination technique that ordinates both species and samples (plots) concurrently (Hill and Gauch 1980). Indirect gradient analysis/ordination obtains axes characterizing major trends of environmental and community variation from calculations based on the sample data (Whittaker 1978). Conversely, direct gradient analysis (DGA) relates species directly to measured environmental factors. DCA therefore does not include analysis of environmental factors (indirect analysis), however, this data is used to help interpret and explain the results.

DCA is based on reciprocal averaging (RA) or correspondence analysis (CA). Its main advantage is that through the detrending process, an 'arch' effect is eliminated that commonly distorts the results of RA and CA. PC-ORD offers several options prior to running the ordination:

* Down-weighting rare species and

* Rescaling of axes.

By down-weighting rare species in DCA, the abundances of species rarer than

\section{Fmax $/ 5$}

(where Fmax is the frequency of the most common species)

are down weighted in a relative amount to their frequency. Species that are more common than Fmax/5 are not down weighted (McCune and Mefford 1999). This option was selected for the analysis to capture the influence of less prevalent species in the sandhills plant community composition without overly distorting the results.

Another option presented in DCA is the rescaling of axes. Another drawback to CA (aside from the arch effect) is that the axis extremes can be condensed. In particular the distances between samples along an axis may not reflect the actual variation in species composition. This compression of the ends of the gradients is corrected in DCA by nonlinear rescaling. The non-linear rescaling is based on the average standard deviation of species turnover and follows the original version of DECORANA in multiplying the standard deviations by 100 and shifting the scales such that all scores are positive (McCune and Mefford 1999). This option was selected, using the default values in PCORD v.4.20 to eliminate the compression of species/samples at the extremes of axes. 


\subsection{Taxonomic Considerations}

Plant scientific names used in this report correspond to Moss (1983) and Alberta Environmental Protection (1993). However, there is a discrepancy between these references and those used for the classification of ecological communities used by Natureserve, which follows $A$ synonymized checklist and atlas with biological attributes for the vascular flora of the United States, Canada and Greenland (Kartesz 1999). A summary of the discrepancies is provided in Table 2.

Table 2. Synonymy of plant species for the Pakowki Sandhills.

\begin{tabular}{|l|l|l|}
\hline \multicolumn{1}{|c|}{$\begin{array}{c}\text { Alberta Environmental } \\
\text { Protection (1993) }\end{array}$} & \multicolumn{1}{|c|}{ Kartesz (1999) / Natureserve (2002) } & \multicolumn{1}{c|}{ Common Name } \\
\hline Agropyron dasystachyum & Elymus lanceolatus spp. lanceolatus & Northern wheatgrass \\
\hline Agropyron sibiricum & Agropyron fragile & Sibirian wheatgrass \\
\hline Agropyron smithii & Pascopyrum smithii & western wheatgrass \\
\hline Carex lanuginosa & Carex pellita & Wooly sedge \\
\hline Coryphantha vivipara & Escobaria vivipara & Cushion cactus \\
\hline Eurotia lanata & Krascheninnikovia lanata & Winter fat \\
\hline Franseria acanthicarpa & Ambrosia acanthicarpa & Bur ragweed \\
\hline Helianthus subrhomboideus & Helianthus pauciflorus ssp. subrhomboideus & Rhombic leaved sunflower \\
\hline Lactuca pulchella & Lactuca tatarica var. pulchella & Common blue lettuce \\
\hline Lygodesmia rostrata & Shinnersoseris rostrata & Annual skeletonweed \\
\hline Oryzopsis hymenoides & Achnatherum hymenoides & Indian rice grass \\
\hline Smilacina stellata & Maianthemum stellata & Star-flowered Solomon's seal \\
\hline Stipa comata & Hesperostipa comata & Needle and thread \\
\hline
\end{tabular}

The primary concern regarding the use of synonymous species names, is when searching Natureserve for ecological communities, using scientific plant names other than those of Kartesz (1999) will yield false results. For example, searching for Oryzopsis hymenoides within ecological communities will return no similar plant community types. However, searching for Achnatherum hymenoides will return numerous related alliances and associations.

Furthermore, no attempt to standardize species and community names was made when referencing other literature. Many recent reports from the United States describe community types using Kartesz (1999) as the taxonomic reference. As such, community names described in this report may not be completely synonymous with those stated in the literature, although they are referring to the same species. A glossary relating scientific and common plant species names is provided in Appendix 1.

\subsection{Cross-referencing of Proposed Community Types to Literature}

Based on the review of existing literature and the development of a preliminary classification of sandhill community types, two cross-reference tables were developed. The first table cross-referenced proposed community types with similar community types previously described for Alberta. The second table cross-referenced proposed community types with similar community types identified in other jurisdictions, including Saskatchewan, Montana, Idaho, Wyoming, North and South Dakota and Nebraska. In both tables, the proposed community types are also given a similarity rating to the previously identified community types, based on a scale provided by Corns (1983) and also recently used by Strong (2002). The tables facilitated the identification of similar types as well as the identification of information gaps. 


\subsection{Community Classification System}

Community classification for the Pakowki Sandhills followed the hierarchical guidelines documented in the International Classification of Ecological Communities: Terrestrial Vegetation of the United States: Volume 1 - The National Classification System: Development, Status and Application (Grossman et al. 1998). The classification system outlined in this publication organizes terrestrial vegetation into five physiognomic and two floristic levels, as shown in Table 3.

Table 3. Hierarchical levels and definitions for the ICEC terrestrial vegetation classification system, adapted from (Grossman et al. 1998).

\begin{tabular}{|c|c|c|c|}
\hline & Hierarchical Level & Definition & Levels or Examples \\
\hline \multirow{5}{*}{ 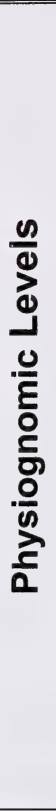 } & Formation Class & $\begin{array}{l}\text { Formation class is defined } \\
\text { based on the vegetation } \\
\text { structure of the dominant, } \\
\text { uppermost life form }\end{array}$ & $\begin{array}{l}\text { 1. Forest/Woodland: Trees with crowns overlapping }(25-99 \% \\
\text { cover) } \\
\text { 2. Shrubland: Shrubs generally }>0.5 \mathrm{~m} \text { height forming }>25 \% \\
\text { cover. } \\
\text { 3. Dwarf-Shrubland: Shrubs }<0.5 \mathrm{~m} \text { height forming }>25 \% \text { cover. } \\
\text { 4. Herbaceous: Graminoids, ferns and forbs dominant. } \\
\text { 5. Non-vascular: Bryophytes, lichens and algae dominant. } \\
\text { 6. Sparse: Abiotic substrate dominant }\end{array}$ \\
\hline & Formation Subclass & $\begin{array}{l}\text { Subclass is based on the } \\
\text { growth-form } \\
\text { characteristics of the } \\
\text { dominant life form, } \\
\text { predominantly leaf } \\
\text { phenology }\end{array}$ & $\begin{array}{l}\text { Evergreen, deciduous, mixed-deciduous for Forest/Woodland, } \\
\text { Shrubland and Dwarf-Shrubland Classes. Perennial and Annual } \\
\text { for the Herbaceous class. Substrate characteristics (e.g. rock, } \\
\text { sand, cobbles, etc.) are used for the Sparse vegetation class. }\end{array}$ \\
\hline & Formation Group & $\begin{array}{l}\text { Group is defined based on } \\
\text { leaf characters, the } \\
\text { presence of a woody } \\
\text { stratum or topographic } \\
\text { position. }\end{array}$ & $\begin{array}{l}\text { Broad leaf or needle leaf used for Forest/Woodland, Shrubland } \\
\text { and Dwarf-Shrubland classes. Presence of a woody stratum } \\
\text { separates groups in Herbaceous and Non-vascular classes. Sparse } \\
\text { vegetation communities are separated based on topographic } \\
\text { position (e.g. shore, cliffs, dunes, etc). }\end{array}$ \\
\hline & $\begin{array}{l}\text { Formation } \\
\text { Subgroup }\end{array}$ & $\begin{array}{l}\text { Subgroup is defined based } \\
\text { on the level of } \\
\text { anthropogenic disturbance. }\end{array}$ & $\begin{array}{l}\text { All groups divide each community type into a Natural/Near } \\
\text { Natural, Semi-Natural or Planted/Cultivated subgroup. }\end{array}$ \\
\hline & Formation & $\begin{array}{l}\text { Formations represent } \\
\text { vegetation types that share } \\
\text { a definite physiognomy or } \\
\text { structure within broadly } \\
\text { defined environmental } \\
\text { factors, landscape positions } \\
\text { or hydrological regimes. }\end{array}$ & e.g. Temperate or sub-polar deciduous shrubland \\
\hline$\underset{0}{0}$ & Alliance & $\begin{array}{l}\text { Alliance is a } \\
\text { physiognomically uniform } \\
\text { groups of plant } \\
\text { associations sharing one or } \\
\text { more diagnostic species, } \\
\text { which as a rule are found } \\
\text { in the uppermost stratum } \\
\text { of the vegetation }\end{array}$ & $\begin{array}{l}\text { e.g. Populus tremuloides - Picea glauca / Linnea borealis Forest } \\
\text { Alliance } \\
\text { e.g. Carex utriculata Herbaceous Alliance }\end{array}$ \\
\hline$\frac{0}{\frac{0}{2}}$ & Association & $\begin{array}{l}\text { Association is the lowest } \\
\text { level of the hierarchy and } \\
\text { is defined as a plant } \\
\text { community type of definite } \\
\text { floristic composition, } \\
\text { uniform habitat conditions } \\
\text { and uniform physiognomy. }\end{array}$ & $\begin{array}{l}\text { Nomenclature is based on the diagnostic species. Species } \\
\text { occurring in the uppermost stratum are listed first (separated by a } \\
\text { hyphen if in the same stratum or a slash if in a different strata) } \\
\text { followed successively by those occurring in lower levels. Within } \\
\text { the same stratum, the order of species names generally reflects } \\
\text { decreasing levels of dominance or constancy. }\end{array}$ \\
\hline
\end{tabular}

\subsection{Evaluation and Assignment of Preliminary Provincial Ranking}

Each community type was evaluated and assigned a preliminary provincial ranking. The ranking system used is based on The Nature Conservancy's species ranking system (Grossman et al. 1994), as used by ANHIC (Allen 2002). The two primary criteria for 
developing community ranks are the total number of occurrences and the total area of each community, range-wide. Measures of geographic range, trends in status and immediate threats to the community's persistence are also considered in ranking. Preliminary ranks range from S1 (rare) to S5 (wide-spread) and are defined in Table 4.

Table 4. Provincial conservation ranks and definitions (adapted from Allen 2002).

\begin{tabular}{|c|l|}
\hline $\begin{array}{c}\text { Preliminary } \\
\text { Rank* }\end{array}$ & Criteria \\
\hline G1(S1) & Five or fewer occurrences or very few remaining hectares \\
\hline G2(S2) & Six to 20 occurrences or few remaining hectares \\
\hline G3(S3) & $\begin{array}{l}\text { 21 to } 100 \text { occurrences. May be rare and local throughout its range or found locally, } \\
\text { even abundantly, in a restricted range (e.g. a single western province or physiographic } \\
\text { region in the East) }\end{array}$ \\
\hline G4(S4) & $\begin{array}{l}\text { Apparently secure globally (State / Province wide), though it may be quite rare in parts } \\
\text { of its range, especially at the periphery. }\end{array}$ \\
\hline G5(S5) & $\begin{array}{l}\text { Demonstrably secure globally (State / Province wide) thought it might be quite rare in } \\
\text { parts of its range, especially at the periphery. }\end{array}$ \\
\hline GU(SU) & Status is uncertain \\
\hline GH(SH) & $\begin{array}{l}\text { Historic. Presumed eliminated in the province with little or no likelihood that it will be } \\
\text { rediscovered. There may be the potential for restoration. }\end{array}$ \\
\hline GX(SX) & $\begin{array}{l}\text { Believed to be eliminated throughout its range, with virtually no likelihood that it will } \\
\text { be rediscovered (e.g. American Chestnut Forest) }\end{array}$ \\
\hline GP(SP) & Potentially exists. Further documentation needed. \\
\hline G?(S?) & Element is not yet ranked. \\
\hline MODIFIERS & $\begin{array}{l}\text { Can be added to any global rank to denote questionable taxonomy (e.g. G2Q= 6 to 20 } \\
\text { known occurrences but questions exist concerning the classification of this type). }\end{array}$ \\
\hline Q & $\begin{array}{l}\text { Can be added to any rank to denote an inexact numeric rank (e.g. G1? = Believed to be } \\
5 \text { or less occurrences but some doubt still exists concerning status). }\end{array}$ \\
\hline ? & $\begin{array}{l}\text { *Ranks can be combined to indicate a range (e.g. G2G3 = May be between 6 to 100 occurrences } \\
\text { throughout range but the exact status is uncertain). Combined ranks indicate a larger margin of error } \\
\text { than ranks assigned a "?" qualifier. }\end{array}$ \\
\hline
\end{tabular}

Where information was available, plant communities from the Pakowki Sandhills were compared with any similar communities found within the subregion, in other regions of the province, or from other provinces or states.

\subsection{Results and Discussion}

\subsection{Vegetation Data Analysis and Classification}

Results from the agglomerative clustering methods were examined to determine the ecological meaning of the clusters and interpret the community types (Figure 1). Examination of the resulting dendrogram revealed numerous small groups. Several community types were consistently clustered together, particularly: 
Plots with high Prunus virginiana cover (plots 10, 13, 14 and 34)

- Plots with high Rosa woodsii cover (and little grass cover) (plots 5, 16 and 26)

* Plots with high Salix amygdaloides - Rosa woodsii cover (plots 27 and 28)

Plots with high Salix exigua / Glycyrrhiza lepidota cover (plots 2 and 7)

- Plots with high Elaeagnus commutata cover (plots 11, 18 and 32)

* Plots with high Rumex venosus cover (plots 6 and 30)

SAND DUNE COMMUNITIES
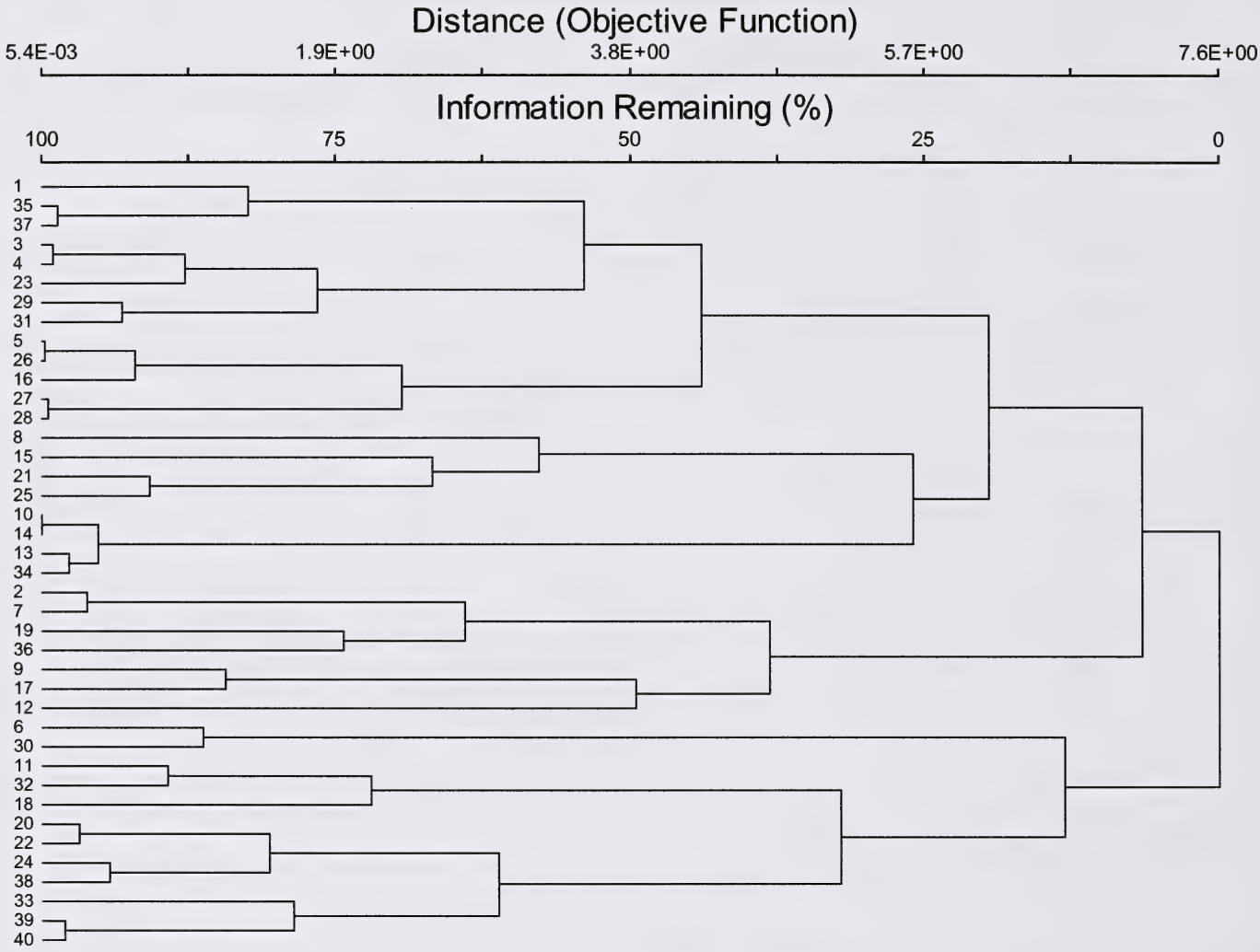

Figure 1. Cluster analysis of 40 plots collected July 2002. Linkage method: Farthest Neighbour. Distance Measure: Bray-Curtis (Sorensen). Percent Chaining=2.82\%. 
However, other community types, particularly grass and forb-dominated communities could not be easily discerned based on the cluster analysis alone.

Detrended correspondence analysis (DCA) helped to group plots with similar species compositions and also confirmed some of the community types observed from the grouping of plots in the cluster analysis. Approximately 17 groups were recognized based on the ordination of plots and species, with several 'groups' comprising only one sample plot as shown in Figure 2 and Figure 3. Output from the PC-ORD DCA is provided in Appendix 2.

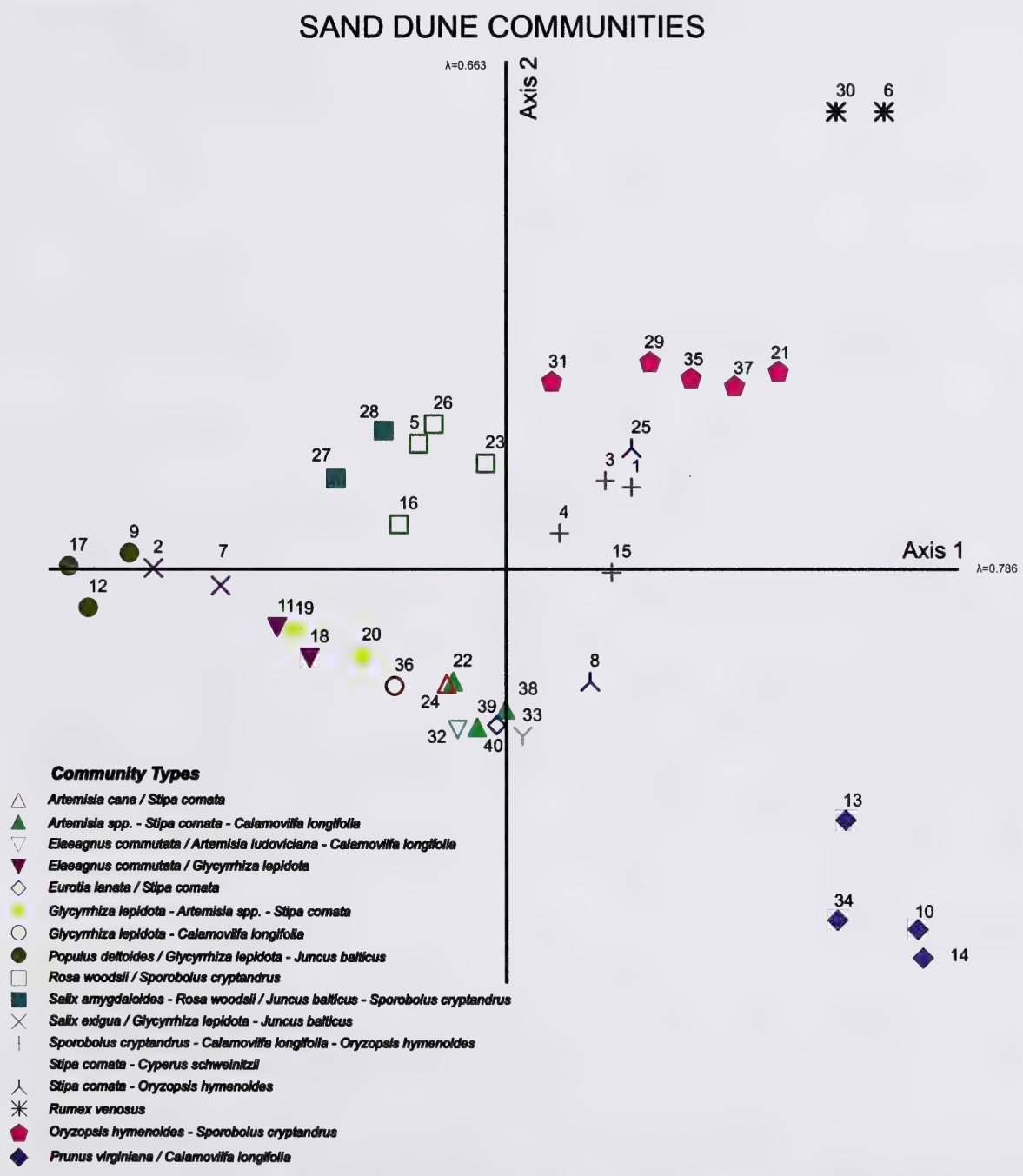

Figure 2. Ordination diagram based on Detrended Correspondence Analysis (DCA) of 40 plots collected in July 2002. Total variance in the species data (inertia) $=6.2854$. Eigenvector $1=0.786$ or $12.5 \%$ of the total variance in the species data. Eigenvector $2=$ 0.663 or $10.54 \%$ of the total variance in the species data. 


\section{SAND DUNE COMMUNITIES}

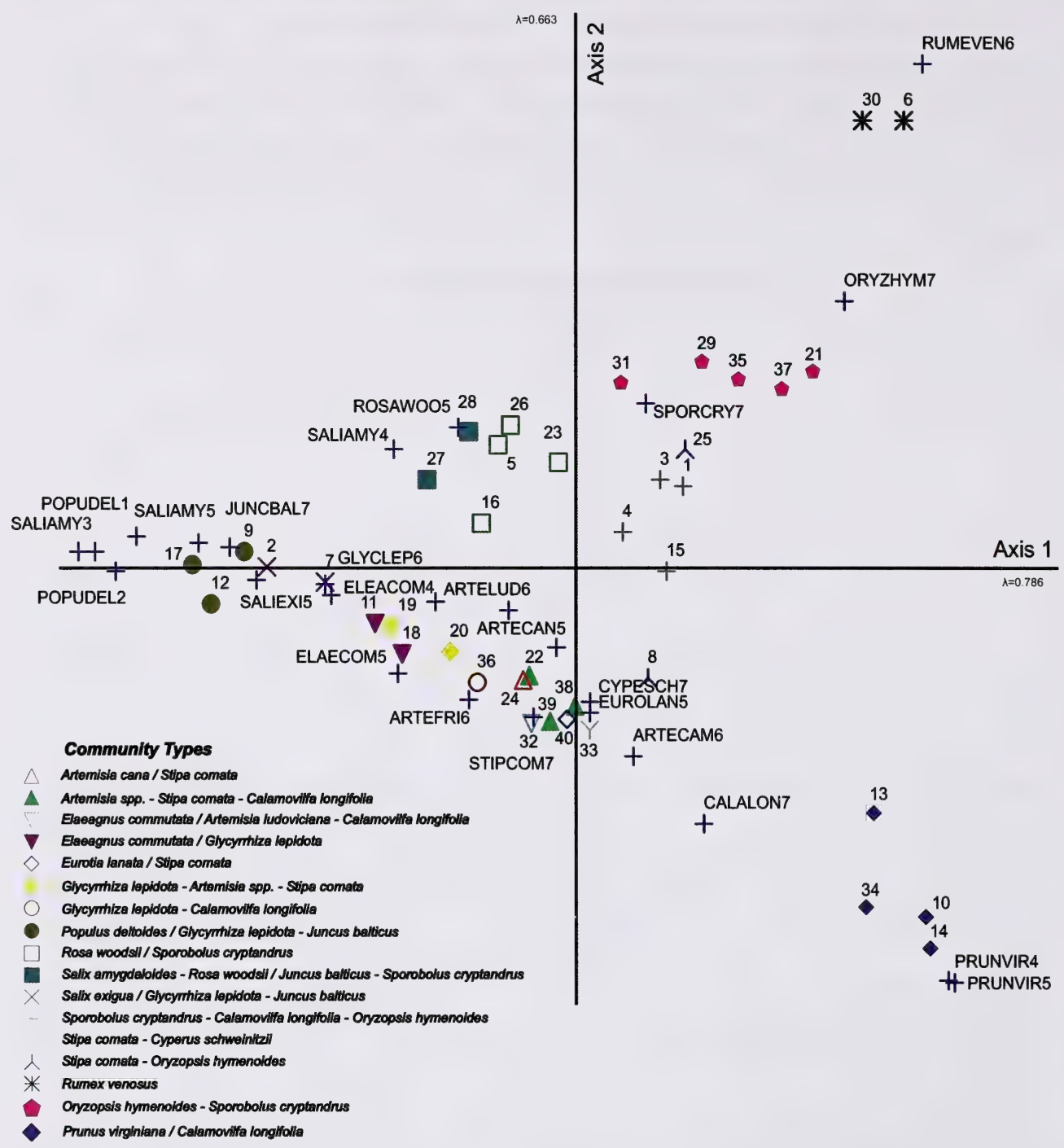

Figure 3. Biplot based on Detrended Correspondence Analysis (DCA) of sample plots (40) and plant species (65) collected at the Pakowki Sandhills in July 2002.

Data were summarized for the resulting community types, including mean percent cover of plant species and surface substrates, standard deviation and standard error and species prominence. This information is presented in section 3.2.1. 


\subsection{Preliminary Classification of Community Types}

A total of 17 plant communities (associations) were found to occur in the Pakowki Sandhills. The majority of communities were ranked SU or S2S3. Community types included all classes, except Non-Vascular and are presented in Table 5 by major physiognomic level.

Table 5. Plant community types (associations) found in the Pakowki Sandhills.

\begin{tabular}{|c|c|}
\hline Terrestrial & Associations \\
\hline \multicolumn{2}{|l|}{ Forest/Woodland } \\
\hline \multicolumn{2}{|l|}{ Deciduous Forest/Woodland } \\
\hline \multicolumn{2}{|l|}{ Cold Deciduous Forest/Woodland } \\
\hline \multicolumn{2}{|l|}{ Natural / Near Natural } \\
\hline \multicolumn{2}{|l|}{$\begin{array}{l}\text { Temperate or subpolar cold deciduous } \\
\text { forest/woodland }\end{array}$} \\
\hline Populus deltoides Forest/Woodland Alliance & Populus deltoides / Glycyrrhiza lepidota - Juncus balticus \\
\hline \multicolumn{2}{|l|}{ Shrubland } \\
\hline \multicolumn{2}{|l|}{ Deciduous Shrubland } \\
\hline \multicolumn{2}{|l|}{ Cold Deciduous Shrubland } \\
\hline \multicolumn{2}{|l|}{ Natural / Near Natural } \\
\hline \multicolumn{2}{|l|}{ Temperate cold deciduous shrubland } \\
\hline Rosa woodsii Shrubland Alliance & Rosa woodsii / Sporobolus cryptandrus \\
\hline Salix amygdaloides Shrubland Alliance & $\begin{array}{l}\text { Salix amygdaloides - Rosa woodsii / Juncus balticus - } \\
\text { Sporobolus cryptandrus }\end{array}$ \\
\hline Elaeagnus commutata Shrubland Alliance & Elaeagnus commutata / Glycyrrhiza lepidota \\
\hline Elaeagnus commutata Shrubland Alliance & $\begin{array}{l}\text { Elaeagnus commutata / Artemisia ludoviciana - Calamovilfa } \\
\text { longifolia }\end{array}$ \\
\hline Prunus virginiana Shrubland Alliance & Prunus virginiana / Calamovilfa longifolia \\
\hline \multicolumn{2}{|l|}{ Dwarf shrubland } \\
\hline \multicolumn{2}{|l|}{ Evergreen dwarf shrubland } \\
\hline \multicolumn{2}{|l|}{ Extremely xeromorphic evergreen dwarf shrubland } \\
\hline \multicolumn{2}{|l|}{ Natural / Near Natural } \\
\hline \multicolumn{2}{|l|}{$\begin{array}{l}\text { Extremely xeromorphic evergreen subdesert } \\
\text { dwarf-shrubland }\end{array}$} \\
\hline Eurotia lanata Dwarf-Shrubland Alliance & Eurotia lanata / Stipa comata - Calamovilfa longifolia \\
\hline \multicolumn{2}{|l|}{ Herbaceous Vegetation } \\
\hline \multicolumn{2}{|l|}{ Perennial forb vegetation } \\
\hline \multicolumn{2}{|l|}{ Temperate or subpolar perennial forb } \\
\hline \multicolumn{2}{|l|}{ Natural / Near Natural } \\
\hline \multicolumn{2}{|l|}{ Low temperate or subpolar perennial forb } \\
\hline Glycyrrhiza lepidota Herbaceous Alliance & Salix exigua / Glycyrrhiza lepidota - Juncus balticus \\
\hline Glycyrrhiza lepidota Herbaceous Alliance & Glycyrrhiza lepidota - Calamovilfa longifolia \\
\hline $\begin{array}{l}\text { Glycyrrhiza lepidota }- \text { Artemisia spp. } \\
\text { Herbaceous Alliance }\end{array}$ & Glycyrrhiza lepidota - Artemisia spp. - Stipa comata \\
\hline Rumex venosus Herbaceous Alliance & Rumex venosus \\
\hline \multicolumn{2}{|l|}{ Herbaceous Vegetation } \\
\hline \multicolumn{2}{|l|}{ Perennial Graminoid Vegetation } \\
\hline \multicolumn{2}{|l|}{ Temperate or sub-polar grasslands } \\
\hline \multicolumn{2}{|l|}{ Natural / Near Natural } \\
\hline \multicolumn{2}{|l|}{$\begin{array}{l}\text { Medium-tall bunch temperate or sub-polar } \\
\text { grasslands }\end{array}$} \\
\hline Oryzopsis hymenoides Herbaceous Alliance & Oryzopsis hymenoides - Sporobolus cryptandrus \\
\hline Stipa comata Bunch Herbaceous Alliance & Stipa comata - Oryzopsis hymenoides \\
\hline
\end{tabular}


Table 5 (cont.). Plant community types (associations) found in the Pakowki Sandhills.

\begin{tabular}{|c|c|}
\hline Terrestrial & Associations \\
\hline \multicolumn{2}{|l|}{ Herbaceous Vegetation } \\
\hline \multicolumn{2}{|l|}{ Perennial Graminoid Vegetation } \\
\hline \multicolumn{2}{|l|}{ Temperate or sub-polar grassiands } \\
\hline \multicolumn{2}{|l|}{ Natural / Near Natural } \\
\hline \multicolumn{2}{|l|}{$\begin{array}{l}\text { Medium-tall sod temperate or sub-polar } \\
\text { grassiands }\end{array}$} \\
\hline Stipa comata Bunch Herbaceous Alliance & Artemisia spp. - Stipa comata - Calamovilfa longifolia \\
\hline \multicolumn{2}{|l|}{ Herbaceous Vegetation } \\
\hline \multicolumn{2}{|l|}{ Perennial Graminoid Vegetation } \\
\hline \multicolumn{2}{|l|}{ Temperate or sub-polar grasslands } \\
\hline \multicolumn{2}{|l|}{ Natural / Near Natural } \\
\hline \multicolumn{2}{|l|}{ Tall sod temperate or sub-polar grasslands } \\
\hline Calamovilfa longifolia Herbaceous Alliance & Stipa comata - Calamovilfa longifolia - Cyperus schweinitzii \\
\hline \multicolumn{2}{|l|}{ Herbaceous Vegetation } \\
\hline \multicolumn{2}{|l|}{ Perennial graminoid vegetation } \\
\hline \multicolumn{2}{|l|}{$\begin{array}{l}\text { Temperate or subpolar grassland with a sparse shrub } \\
\text { layer }\end{array}$} \\
\hline \multicolumn{2}{|l|}{ Natural / Near Natural } \\
\hline \multicolumn{2}{|l|}{ Medium-tall temperate or subpolar grassland } \\
\hline Sporobolus cryptandrus Herbaceous Alliance & $\begin{array}{l}\text { Sporobolus cryptandrus - Calamovilfa longifolia - Oryzopsis } \\
\text { hymenoides }\end{array}$ \\
\hline \multicolumn{2}{|l|}{ Herbaceous Vegetation } \\
\hline \multicolumn{2}{|l|}{ Perennial graminoid vegetation } \\
\hline \multicolumn{2}{|l|}{$\begin{array}{l}\text { Temperate or subpolar grassland with a sparse shrub } \\
\text { layer }\end{array}$} \\
\hline \multicolumn{2}{|l|}{ Natural / Near Natural } \\
\hline \multicolumn{2}{|l|}{$\begin{array}{l}\text { Medium-tall temperate or subpolar grassland with } \\
\text { a sparse needle-leaved or microphyllous evergreen } \\
\text { shrub layer }\end{array}$} \\
\hline Artemisia cana Shrub Herbaceous Alliance & Artemisia cana / Stipa comata \\
\hline
\end{tabular}

\subsubsection{Description of Community Types}

A summary of the 17 community types (associations) found in the Pakowki Sandhills is described below. Representative photos for each community type are provided as Plates $1-17$. A full listing of species codes is provided in Appendix 3. Table 6 provides an explanation of strata codes, which follow the seven-letter species code.

Table 6. Numeric codes used to define different vegetation structural strata.

\begin{tabular}{c|l}
\hline Code & Stratum \\
\hline 1 & Main Canopy Trees \\
2 & Understorey Trees \\
3 & Tall Shrubs \\
4 & Medium Shrubs \\
5 & Low Shrubs \\
6 & Forbs / Herbs \\
7 & Grasses \\
\hline
\end{tabular}




\subsubsection{Populus deltoides / Glycyrrhiza lepidota - Juncus balticus Association}

Western cottonwood / wild licorice - wire rush

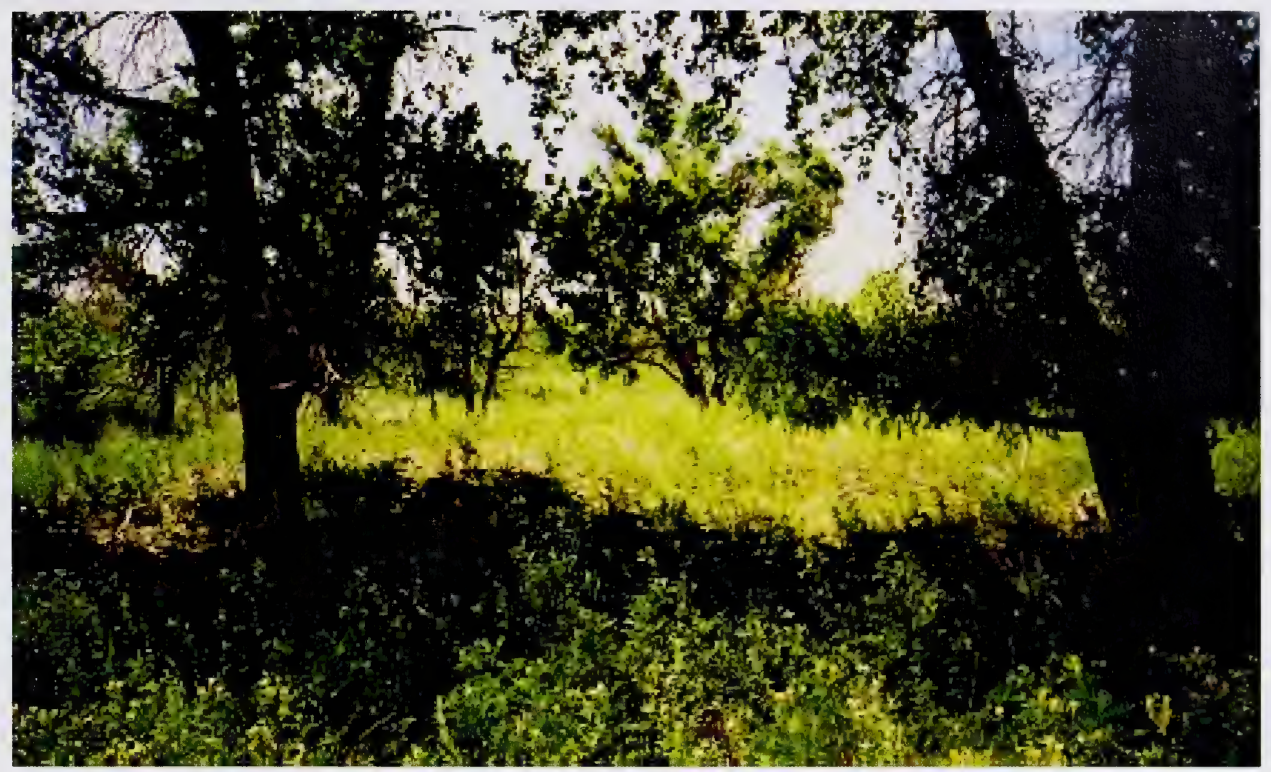

Plate 1. Populus deltoides / Glycyrrhiza lepidota - Juncus balticus community type (Western cottonwood / wild licorice - wire rush) Plot 17.

This community was dominated by Populus deltoides, but occurred in association with Populus tremuloides or Salix amygdaloides, in tree form. Understorey shrubs such as Rosa woodsii, Salix exigua and Elaeagnus commutata were occasionally present, though they typically did not exceed more than $5 \%$ cover. Glycyrrhiza lepidota was present at all sites and ranged in cover between $2 \%$ and $20 \%$ cover. Thermopsis rhombifolia, Chenopodium fremontii, C. pratericola, Artemisia ludoviciana and Solidago missouriensis were also typical forb species. Juncus balticus was the dominant graminoid species, although Poa pratensis and Bouteloua gracilis were also commonly present. Table 7 summarizes the species composition, mean percent cover, standard error and species prominence for this community type. Species prominence was calculated using the following formula:

$$
\text { mean } \% \text { cover } x \sqrt{\% \text { presence }}
$$


Table 7. Summary statistics for the Populus deltoides / Glycyrrhiza lepidota-Juncus balticus community type $(\mathrm{n}=3)$.

\begin{tabular}{lcccc}
\hline \hline Species & $\begin{array}{c}\text { Mean } \% \\
\text { Cover }\end{array}$ & $\begin{array}{c}\text { Standard } \\
\text { Error }\end{array}$ & Presence & Prominence \\
\hline \hline POPUDEL2 & 23.33 & 23.33 & 1 & 40.4 \\
\hline POPUDEL1 & 36.67 & 17.64 & 3 & 36.7 \\
\hline POPUTRE2 & 13.33 & 13.33 & 1 & 23.1 \\
\hline SALIAMY3 & 8.33 & 8.33 & 1 & 14.4 \\
\hline GLYCLEP6 & 12.33 & 5.36 & 3 & 12.3 \\
\hline JUNCBAL7 & 11.67 & 4.41 & 3 & 11.7 \\
\hline CHENPRA6 & 2.67 & 1.20 & 3 & 2.7 \\
\hline THERRHO6 & 2.00 & 1.53 & 2 & 2.4 \\
\hline POAPRA7 & 2.00 & 1.53 & 2 & 2.4 \\
\hline CHENFRE6 & 1.67 & 0.33 & 3 & 1.7 \\
\hline DESCSOP6 & 1.00 & 0.50 & 3 & 1.0 \\
\hline ROSAWOO5 & 0.67 & 0.33 & 2 & 0.8 \\
\hline SOLIMIS6 & 0.67 & 0.17 & 3 & 0.7 \\
\hline ARTELUD6 & 0.50 & 0.29 & 2 & 0.6 \\
\hline ELAECOM5 & 0.33 & 0.33 & 1 & 0.6 \\
\hline BOUTGRA7 & 0.33 & 0.33 & 1 & 0.6 \\
\hline \hline
\end{tabular}

\begin{tabular}{lcccc}
\hline \hline Species & $\begin{array}{c}\text { Mean \% } \\
\text { Cover }\end{array}$ & $\begin{array}{c}\text { Standard } \\
\text { Error }\end{array}$ & Presence & Prominence \\
\hline \hline TARAOFF6 & 0.33 & 0.17 & 2 & 0.4 \\
\hline CRYPFEN6 & 0.33 & 0.17 & 2 & 0.4 \\
\hline SALIEXI5 & 0.17 & 0.17 & 1 & 0.3 \\
\hline CLEOSER6 & 0.17 & 0.17 & 1 & 0.3 \\
\hline HELISUB6 & 0.17 & 0.17 & 1 & 0.3 \\
\hline EQUIHYE6 & 0.17 & 0.17 & 1 & 0.3 \\
\hline TRAGDUB6 & 0.17 & 0.17 & 1 & 0.3 \\
\hline CHENSUB6 & 0.17 & 0.17 & 1 & 0.3 \\
\hline SMILSTE6 & 0.17 & 0.17 & 1 & 0.3 \\
\hline SOLICAN6 & 0.17 & 0.17 & 1 & 0.3 \\
\hline HETEVIL6 & 0.17 & 0.17 & 1 & 0.3 \\
\hline ELYMCAN7 & 0.17 & 0.17 & 1 & 0.3 \\
\hline \hline DECAY9 & 0.50 & 0.00 & & \\
\hline MINERAL9 & 11.67 & 4.41 & & \\
\hline ORG9 & 50.00 & 5.77 & & \\
\hline \hline
\end{tabular}

This community was found in localized depressions between dunes and in more open sand plain areas. Soils were sandy, but with some organic matter buildup on the soil surface. A summary of site data is provided in Table 8 .

Table 8. Summary of site data for the Populus deltoides / Glycyrrhiza lepidota - Juncus balticus community type.

\begin{tabular}{ccccccccc}
\hline \hline Plot & Elevation & Aspect & Slope & Drainage & $\begin{array}{c}\text { Site } \\
\text { Position }\end{array}$ & Surface Shape & $\begin{array}{c}\text { Moisture } \\
\text { Regime }\end{array}$ & Nutrient Regime \\
\hline \hline 9 & 870 & $\mathrm{n} / \mathrm{a}$ & 0 & Rapid & Level & Straight & Submesic & Mesotrophic \\
\hline 12 & 863 & $\mathrm{n} / \mathrm{a}$ & 0 & Rapid & Level & Straight & Subxeric & Submesotrophic \\
\hline 17 & 864 & $\mathrm{n} / \mathrm{a}$ & 0 & Rapid & Level & Straight & Subxeric & Submesotrophic \\
\hline \hline
\end{tabular}

This community was typically well used by both livestock and wild ungulates, with many pellet groups observed. 


\subsubsection{Rosa woodsii / Sporobolus cryptandrus Association}

Common wild rose / sand dropseed

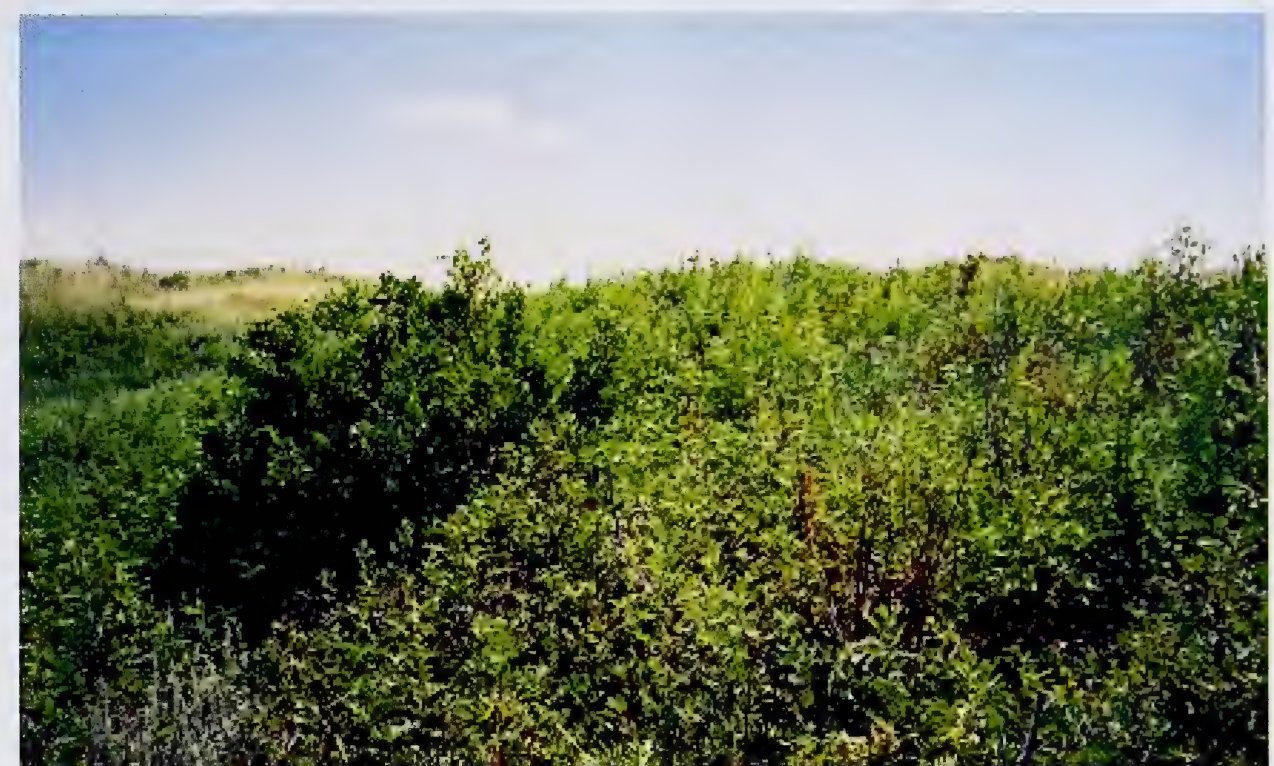

Plate 2. Rosa woodsii / Sporobolus cryptandrus community type (Common wild rose / sand dropseed) Plot 16.

This community was dominated by Rosa woodsii in the shrub layer, although other species such as Ribes oxyacanthoides and Salix exigua were also present. Glycyrrhiza lepidota was the dominant forb species with a cover ranging up to $30 \%$. Other typical forb species included Chenopodium pratericola, C. fremontii and Heterotheca villosa. Sporobolus cryptandrus was the dominant graminoid species, although Calamovilfa longifolia was quite common, while Oryzopsis hymenoides was occasionally present. Table 9 summarizes the species composition, mean percent cover, standard error and species prominence for this community type. 
Table 9. Summary statistics for the Rosa woodsii / Sporobolus cryptandrus community type $(n=4)$.

\begin{tabular}{lcccc}
\hline \hline Species & $\begin{array}{c}\text { Mean \% } \\
\text { Cover }\end{array}$ & $\begin{array}{c}\text { Standard } \\
\text { Error }\end{array}$ & Presence & Prominence \\
\hline \hline ROSAWOO5 & 42.50 & 12.08 & 4 & 42.5 \\
\hline SPORCRY7 & 12.50 & 4.08 & 4 & 12.5 \\
\hline GLYCLEP6 & 7.75 & 7.01 & 3 & 8.9 \\
\hline RIBEOXY5 & 2.50 & 2.50 & 1 & 5.0 \\
\hline ARTELUD6 & 2.50 & 2.50 & 1 & 5.0 \\
\hline CALALON7 & 3.00 & 2.38 & 2 & 4.2 \\
\hline THERRHO6 & 0.50 & 0.50 & 1 & 1.0 \\
\hline CHENPRA6 & 0.75 & 0.35 & 4 & 0.8 \\
\hline SOLIMIS6 & 0.50 & 0.29 & 2 & 0.7 \\
\hline HELIANN6 & 0.38 & 0.24 & 2 & 0.5 \\
\hline ORYZHYM7 & 0.38 & 0.24 & 2 & 0.5 \\
\hline PRUNVIR5 & 0.25 & 0.25 & 1 & 0.5 \\
\hline AGRODAS7 & 0.25 & 0.25 & 1 & 0.5 \\
\hline \hline
\end{tabular}

\begin{tabular}{lcccc}
\hline \hline \multicolumn{1}{c}{ Species } & $\begin{array}{c}\text { Mean } \% \\
\text { Cover }\end{array}$ & $\begin{array}{c}\text { Standard } \\
\text { Error }\end{array}$ & Presence & Prominence \\
\hline \hline AGROSIB7 & 0.25 & 0.25 & 1 & 0.5 \\
\hline CARELAN7 & 0.25 & 0.25 & 1 & 0.5 \\
\hline HETEVIL6 & 0.38 & 0.00 & 4 & 0.4 \\
\hline SALIEXI5 & 0.25 & 0.48 & 2 & 0.4 \\
\hline LYGOROS6 & 0.25 & 0.14 & 2 & 0.4 \\
\hline CHENFRE6 & 0.25 & 0.13 & 3 & 0.3 \\
\hline CORYVIV6 & 0.13 & 0.13 & 1 & 0.3 \\
\hline LATUPUL6 & 0.13 & 0.13 & 1 & 0.3 \\
\hline ELYMCAN7 & 0.13 & 0.13 & 1 & 0.3 \\
\hline CHENSUB6 & 0.13 & 0.14 & 2 & 0.2 \\
\hline RUMEVEN6 & 0.13 & 0.14 & 2 & 0.2 \\
\hline MINERAL9 & 17.50 & 5.95 & & \\
\hline ORG9 & 17.63 & 8.45 & & \\
\hline \hline
\end{tabular}

This community was typically found on the north to southeast, leeward slopes of partially stabilized dunes. Soils were sandy, with some organic matter buildup on the soil surface and some exposed sand. A summary of site data is provided in Table 10.

Table 10. Summary of site data for the Rosa woodsii / Sporobolus cryptandrus community type $(n=4)$.

\begin{tabular}{ccccccccc}
\hline \hline Plot & Elevation & Aspect & Slope & Drainage & Site Position & $\begin{array}{c}\text { Surface } \\
\text { Shape }\end{array}$ & $\begin{array}{c}\text { Moisture } \\
\text { Regime }\end{array}$ & Nutrient Regime \\
\hline \hline 5 & 898 & 104 & 45 & Rapid & Upper Slope & Straight & Xeric & Submesotrophic \\
\hline 16 & 866 & 5 & 18 & Rapid & Middle Slope & Straight & Subxeric & Submesotrophic \\
\hline 23 & 886 & 158 & 10 & Rapid & Middle Slope & Straight & Xeric & Submesotrophic \\
\hline 26 & 883 & 35 & 15 & Rapid & Upper Slope & Straight & Subxeric & Submesotrophic \\
\hline \hline
\end{tabular}




\subsubsection{Salix amygdaloides - Rosa woodsii / Juncus balticus - Sporobolus cryptandrus Association}

Peach-leaf willow - common wild rose / wire rush - sand dropseed

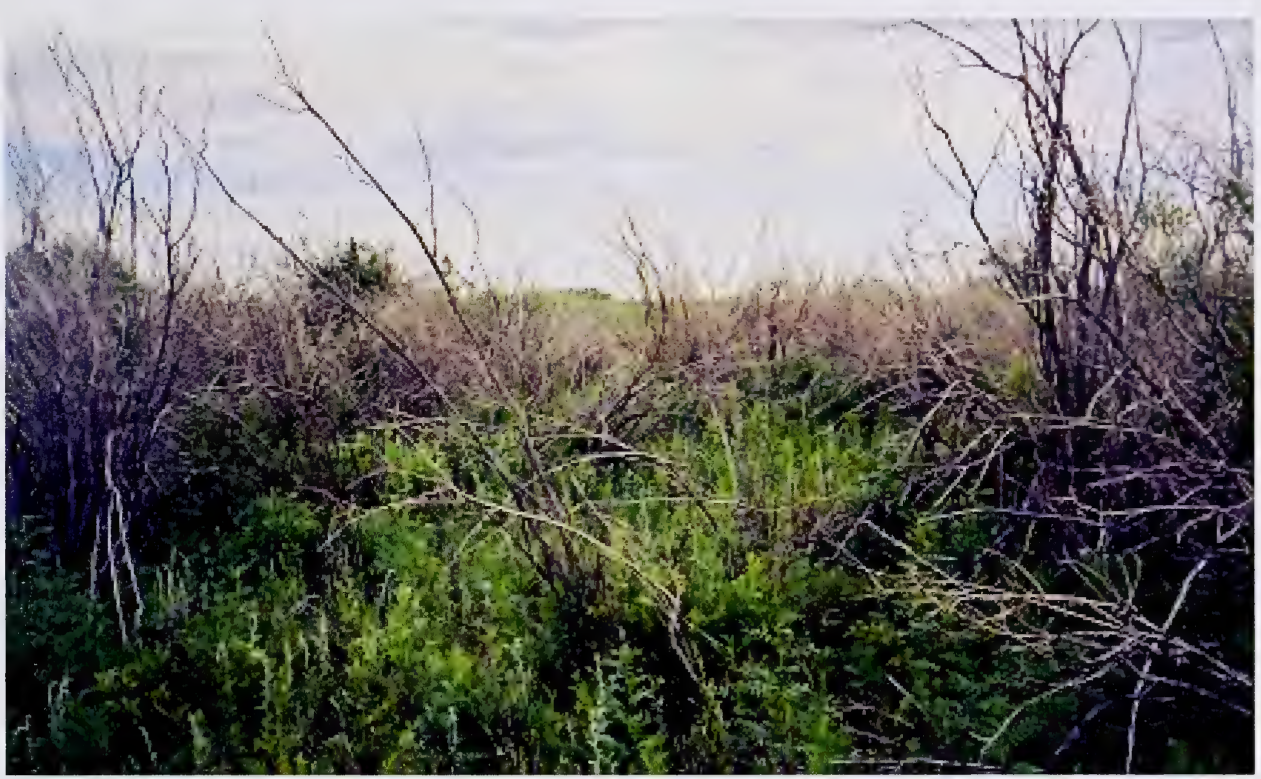

Plate 3. Salix amygdaloides - Rosa woodsii / Juncus balticus - Sporobolus cryptandrus (Peach-leaf willow - common wild rose / wire rush - sand dropseed) Plot 27.

This community was co-dominated by Salix amygdaloides and Rosa woodsii in the shrub layer. Juncus balticus and Sporobolus cryptandrus were the dominant graminoids, although Glycyrrhiza lepidota and Thermopsis rhombifolia constituted a substantial forb cover at certain sites. Other typical species, though with low percent cover, included Prunus virginiana, Chenopodium pratericola and C. fremontii. Table 11 summarizes the species composition, mean percent cover, standard error and species prominence for this community type. 
Table 11. Summary statistics for the Salix amygdaloides - Rosa woodsii / Juncus balticus -Sporobolus cryptandrus community type $(\mathrm{n}=2)$.

\begin{tabular}{lcccc}
\hline \hline Species & $\begin{array}{c}\text { Mean \% } \\
\text { Cover }\end{array}$ & $\begin{array}{c}\text { Standard } \\
\text { Error }\end{array}$ & Presence & Prominence \\
\hline \hline SALIAMY4 & 40.00 & 10.00 & 2 & 40.0 \\
\hline ROSAWOO5 & 40.00 & 0.00 & 2 & 40.0 \\
\hline GLYCLEP6 & 22.50 & 22.50 & 1 & 31.8 \\
\hline SPORCRY7 & 25.00 & 0.00 & 2 & 25.0 \\
\hline THERRHO6 & 22.50 & 2.50 & 2 & 22.5 \\
\hline JUNCBAL7 & 15.00 & 0.00 & 2 & 15.0 \\
\hline PRUNVIR5 & 1.00 & 0.00 & 2 & 1.0 \\
\hline CHENPRA6 & 1.00 & 0.00 & 2 & 1.0 \\
\hline CHENFRE6 & 1.00 & 0.00 & 2 & 1.0 \\
\hline \hline
\end{tabular}

\begin{tabular}{lcccc}
\hline \multicolumn{1}{c}{ Species } & $\begin{array}{c}\text { Mean \% } \\
\text { Cover }\end{array}$ & $\begin{array}{c}\text { Standard } \\
\text { Error }\end{array}$ & Presence & Prominence \\
\hline \hline SOLIMIS6 & 0.50 & 0.50 & 1 & 0.7 \\
\hline AGROSIB7 & 0.50 & 0.50 & 1 & 0.7 \\
\hline POAPRA7 & 0.50 & 0.50 & 1 & 0.7 \\
\hline ELYMCAN7 & 0.50 & 0.50 & 1 & 0.7 \\
\hline DESCSOP6 & 0.50 & 0.00 & 2 & 0.5 \\
\hline TRAGDUB6 & 0.25 & 0.25 & 1 & 0.4 \\
\hline AGTEVIL6 & 0.25 & 0.25 & 1 & 0.4 \\
\hline \hline DECAY9 & 0.25 & 0.25 & 1 & 0.4 \\
\hline MINERAL9 & 12.50 & 2.50 & & \\
\hline \hline
\end{tabular}

This community was found in relatively level sand plain sites, with rapidly to well drained sandy soils. Organic matter buildup on the soil surface was commonly greater than $30 \%$ cover, though exposed sand was still at the soil surface. A summary of site data is provided in Table 12 .

Table 12. Summary of site data for the Salix amygdaloides - Rosa woodsii / Juncus balticus - Sporobolus cryptandrus community type $(\mathrm{n}=2)$.

\begin{tabular}{lcccccccc}
\hline \hline Plot & Elevation & Aspect & Slope & Drainage & Site Position & $\begin{array}{c}\text { Surface } \\
\text { Shape }\end{array}$ & $\begin{array}{c}\text { Moisture } \\
\text { Regime }\end{array}$ & Nutrient Regime \\
\hline \hline 27 & 875 & $\mathrm{n} / \mathrm{a}$ & 0 & Well & Level & Straight & Subxeric & Submesotrophic \\
\hline 28 & 874 & $\mathrm{n} / \mathrm{a}$ & 0 & Rapid & Level & Straight & Xeric & Submesotrophic \\
\hline \hline
\end{tabular}




\subsubsection{Elaeagnus commutata / Glycyrrhiza lepidota Association \\ Silverberry / wild licorice}

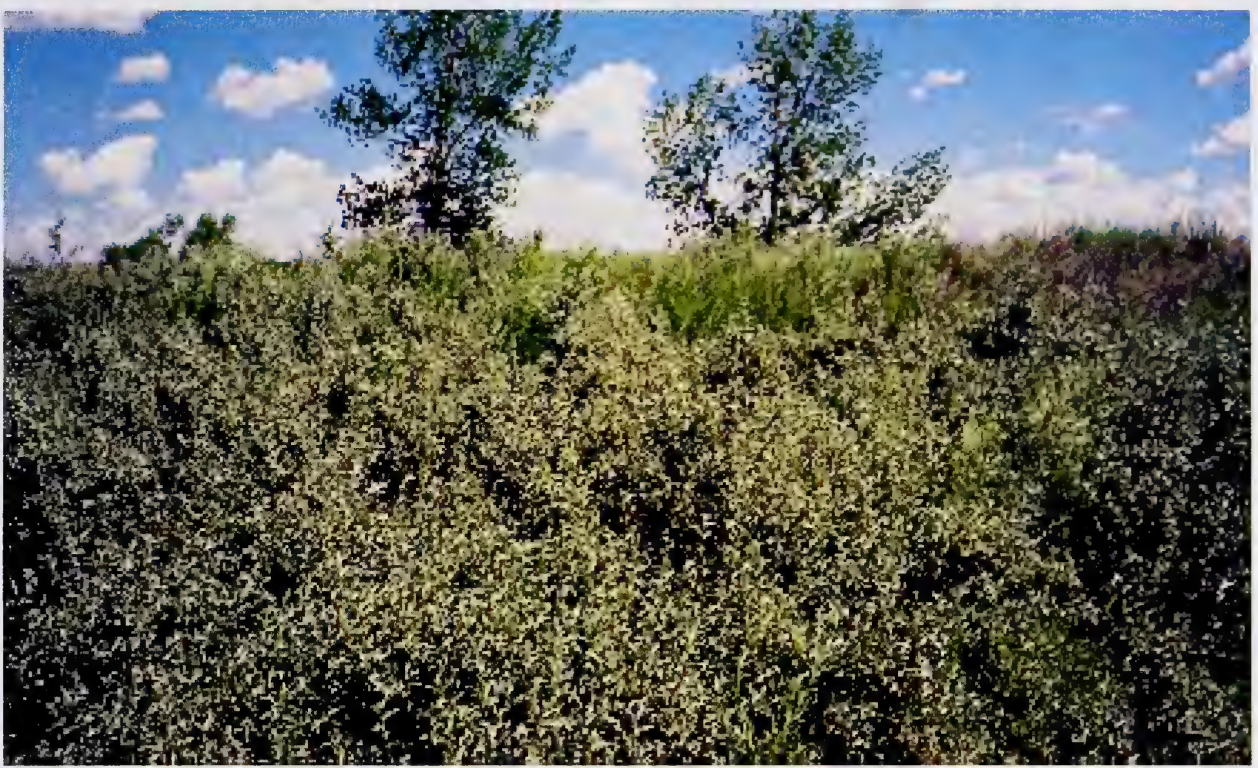

Plate 4. Elaeagnus commutata / Glycyrrhiza lepidota community type (Silverberry / wild licorice) Plot 18.

This community was dominated by Elaeagnus commutata in the mid-shrub-layer. Glycyrrhiza lepidota was the dominant forb, averaging about $15 \%$ cover. Other typical forbs that may have a low percent cover included: Artemisia ludoviciana, Thermopsis rhombifolia, Cryptantha fendleri and Chenopodium pratericola. Juncus balticus was the dominant graminoid species, although other species such as Stipa comata, Koeleria macratha and Agropyron dasystachyum also occurred. Table 13 summarizes the species composition, mean percent cover, standard error and species prominence for this community type. 
Table 13. Summary statistics for the Elaeagnus commutata / Glycyrrhiza lepidota community type $(\mathrm{n}=2)$.

\begin{tabular}{lcccc}
\hline \hline Species & $\begin{array}{c}\text { Mean \% } \\
\text { Cover }\end{array}$ & $\begin{array}{c}\text { Standard } \\
\text { Error }\end{array}$ & Presence & Prominence \\
\hline \hline ELAECOM5 & 50.00 & 15.00 & 2 & 50.0 \\
\hline GLYCLEP6 & 15.00 & 10.00 & 2 & 15.0 \\
\hline SALIEXI5 & 10.00 & 10.00 & 1 & 14.1 \\
\hline JUNCBAL7 & 10.00 & 10.00 & 1 & 14.1 \\
\hline ELEACOM4 & 2.50 & 2.50 & 1 & 3.5 \\
\hline ARTELUD6 & 2.50 & 2.50 & 1 & 3.5 \\
\hline STIPCOM7 & 2.75 & 2.25 & 2 & 2.8 \\
\hline THERRHO6 & 1.50 & 1.50 & 1 & 2.1 \\
\hline POAPRA7 & 1.50 & 1.50 & 1 & 2.1 \\
\hline PRUNVIR5 & 1.00 & 1.00 & 1 & 1.4 \\
\hline SOLIMIS6 & 1.00 & 1.00 & 1 & 1.4 \\
\hline LYGOROS6 & 1.00 & 1.00 & 1 & 1.4 \\
\hline CYPESCH7 & 1.00 & 1.00 & 1 & 1.4 \\
\hline \hline
\end{tabular}

\begin{tabular}{lcccc}
\hline \hline Species & $\begin{array}{c}\text { Mean \% } \\
\text { Cover }\end{array}$ & $\begin{array}{c}\text { Standard } \\
\text { Error }\end{array}$ & Presence & Prominence \\
\hline \hline SPORCRY7 & 1.00 & 1.00 & 1 & 1.4 \\
\hline CALALON7 & 1.00 & 1.00 & 1 & 1.4 \\
\hline KOELMAC7 & 1.25 & 0.75 & 2 & 1.3 \\
\hline AGRODAS7 & 1.25 & 0.75 & 2 & 1.3 \\
\hline CRYPFEN6 & 0.75 & 0.25 & 2 & 0.8 \\
\hline HETEVIL6 & 0.75 & 0.25 & 2 & 0.8 \\
\hline ROSAWOO5 & 0.50 & 0.50 & 1 & 0.7 \\
\hline CHENPRA6 & 0.50 & 0.00 & 2 & 0.5 \\
\hline CHENFRE6 & 0.25 & 0.25 & 1 & 0.4 \\
\hline HELIANN6 & 0.25 & 0.25 & 1 & 0.4 \\
\hline \hline DECAY9 & 0.25 & 0.25 & & \\
\hline MINERAL9 & 15.00 & 5.00 & & \\
\hline ORG9 & 20.00 & 10.00 & & \\
\hline \hline
\end{tabular}

This community types was found in depressional locations on the windward side of dunes, with well-drained soils. Surface organic matter ranged from $10 \%$ to $30 \%$, with only $10 \%$ to $20 \%$ exposed sand at the soil surface. A summary of site data is provided in Table 14.

Table 14. Summary of site data for the Elaeagnus commutata / Glycyrrhiza lepidota community type $(\mathrm{n}=2)$.

\begin{tabular}{lcccccccc}
\hline \hline Plot Elevation & Aspect & Slope & Drainage & Site Position & $\begin{array}{c}\text { Surface } \\
\text { Shape }\end{array}$ & $\begin{array}{c}\text { Moisture } \\
\text { Regime }\end{array}$ & Nutrient Regime \\
\hline \hline 11 & 864 & $\mathrm{n} / \mathrm{a}$ & 0 & Well & Depression & Straight & Subxeric & Mesotrophic \\
\hline 18 & 863 & $\mathrm{n} / \mathrm{a}$ & 0 & Well & Depression & Concave & Subxeric & Submesotrophic \\
\hline \hline
\end{tabular}




\subsubsection{Elaeagnus commutata / Artemisia ludoviciana - Calamovilfa longifolia Association}

Silverberry / prairie sagewort - sand grass

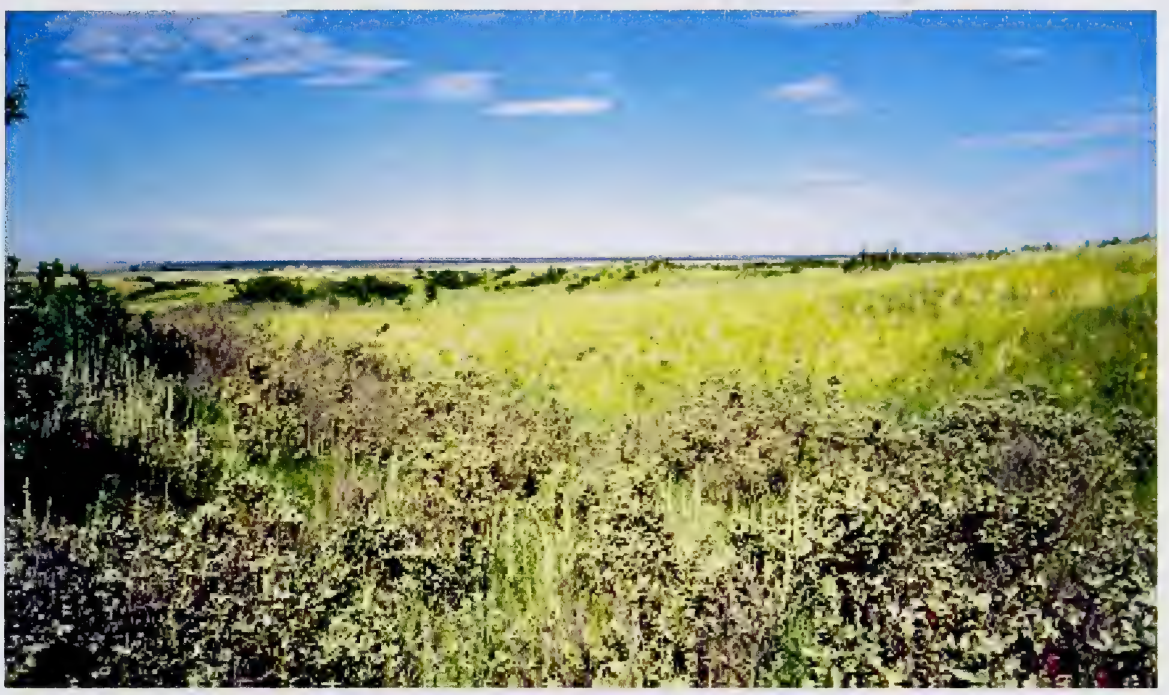

Plate 5. Elaeagnus commutata / Artemisia ludoviciana - Calamovilfa longifolia (Silverberry / prairie sagewort - sand grass) Plot 32.

This community type was dominated by Elaeagnus commutata in the mid-shrub layer. Calamovilfa longifolia was the dominant graminoid, with a cover in this plot of $25 \%$. Other graminoid species included Stipa comata, Cyperus schweinitzii, Sporobolus cryptandrus, Koeleria macrantha and Agropyron dasystachyum generally with covers of less than 1\%. Artemisia ludoviciana was the dominant forb with about $5 \%$ cover, although other species also occur including Solidago missouriensis and Heterotheca villosa. Table 15 summarizes the species composition and percent cover for this community type

Table 15. Summary statistics for the Elaeagnus commutata / Artemisia ludoviciana / Calamovilfa longifolia community type $(\mathrm{n}=1)$.

\begin{tabular}{lc}
\hline \multicolumn{1}{c}{ Species } & Percent Cover \\
\hline \hline ELAECOM5 & 40 \\
\hline CALALON7 & 25 \\
\hline ARTELUD6 & 5 \\
\hline STIPCOM7 & 5 \\
\hline PRUNVIR5 & 1 \\
\hline SOLIMIS6 & 1 \\
\hline HETEVIL6 & 1 \\
\hline CYPESCH7 & 1 \\
\hline SPORCRY7 & 1 \\
\hline CRYPFEN6 & 0.5 \\
\hline \hline
\end{tabular}

\begin{tabular}{lc}
\hline \hline \multicolumn{1}{c}{ Species } & Percent Cover \\
\hline \hline CHENPRA6 & 0.5 \\
\hline CHENFRE6 & 0.5 \\
\hline LYGOROS6 & 0.5 \\
\hline OPUNPOL6 & 0.5 \\
\hline HELIANN6 & 0.5 \\
\hline KOELMAC7 & 0.5 \\
\hline AGRODAS7 & 0.5 \\
\hline \hline DECAY9 & 0.5 \\
\hline MINERAL9 & 10 \\
\hline ORG9 & 5 \\
\hline \hline
\end{tabular}


This community type was found in a small downslope-trending depression or gully on the windward side of a dune. The dune appeared to be quite stabilized with a high vegetative cover. The soil was rapidly drained and the site had a concave surface shape. A summary of site data is provided in Table 16.

Table 16. Summary of site data for the Elaeagnus commutata / Artemisia ludoviciana Calamovilfa longifolia community type $(\mathrm{n}=1)$.

\begin{tabular}{lcccccccc}
\hline \hline Plot Elevation & Aspect & Slope & Drainage & Site Position & $\begin{array}{c}\text { Surface } \\
\text { Shape }\end{array}$ & $\begin{array}{c}\text { Moisture } \\
\text { Regime }\end{array}$ & Nutrient Regime \\
\hline \hline 32 & 879 & 281 & 10 & Rapid & Lower Slope & Concave & Subxeric & Submesotrophic \\
\hline \hline
\end{tabular}




\subsubsection{Prunus virginiana / Calamovilfa longifolia Association} Choke cherry / sand grass

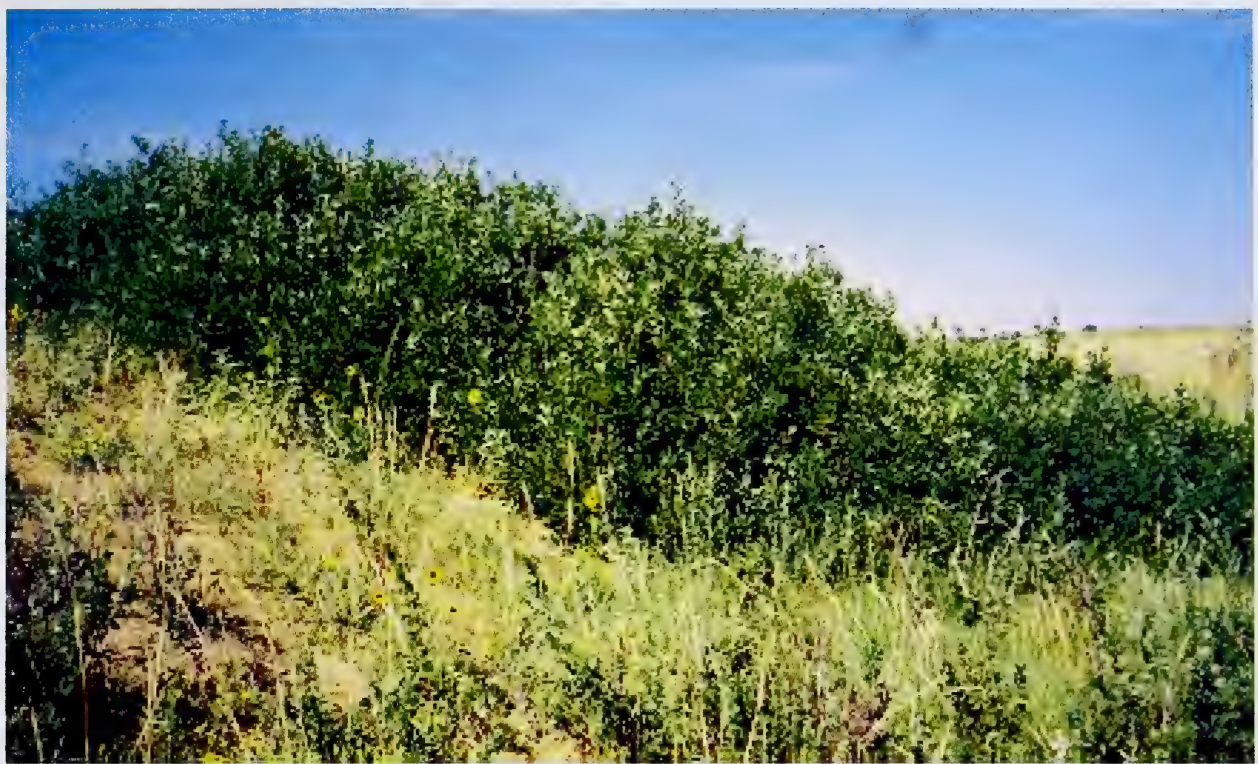

Plate 6. Prunus virginiana / Calamovilfa longifolia community type (Chokecherry / sand grass) Plot 14.

This community type was dominated by Prunus virginiana in the mid- and low-shrub strata. Mean percent cover for this species was greater than $60 \%$ and it formed dense thickets that were impossible to walk through. Calamovilfa longifolia was the dominant graminoid species. Oryzopsis hymenoides was also present at every sample location although with a low percent cover. Sporobolus cryptandrus was also commonly found, although generally with a mean percent cover of less than $3 \%$. Total graminoid cover was typically between 5-10\%. Common forbs included Helianthus annuus, Lygodesmia rostrata, Heterotheca villosa and Cryptantha fendleri. Although these species were present at most sample plot locations, their total percent cover was relatively low. Table 17 summarizes the species composition, mean percent cover, standard error and species prominence for this community type. 
Table 17. Summary statistics for the Prunus virginiana / Calamovilfa longifolia community type $(n=4)$.

\begin{tabular}{lcccc}
\hline \hline Species & $\begin{array}{c}\text { Mean } \% \\
\text { Cover }\end{array}$ & $\begin{array}{c}\text { Standard } \\
\text { Error }\end{array}$ & Presence & Prominence \\
\hline \hline PRUNVIR5 & 63.75 & 8.26 & 4 & 63.8 \\
\hline CALALON7 & 4.25 & 2.14 & 4 & 4.3 \\
\hline PRUNVIR4 & 2.00 & 1.22 & 2 & 2.8 \\
\hline SPORCRY7 & 2.25 & 1.60 & 3 & 2.6 \\
\hline HELIANN6 & 1.75 & 1.09 & 4 & 1.8 \\
\hline ORYZHYM7 & 1.75 & 0.48 & 4 & 1.8 \\
\hline RUMEVEN6 & 1.00 & 0.58 & 2 & 1.4 \\
\hline STIPCOM7 & 1.00 & 0.71 & 2 & 1.4 \\
\hline ARTELUD6 & 0.50 & 0.50 & 1 & 1.0 \\
\hline LYGOROS6 & 0.63 & 0.24 & 3 & 0.7 \\
\hline CHENPRA6 & 0.50 & 0.29 & 2 & 0.7 \\
\hline HETEVIL6 & 0.63 & 0.13 & 4 & 0.6 \\
\hline AGRODAS7 & 0.38 & 0.24 & 2 & 0.5 \\
\hline CHENFRE6 & 0.25 & 0.25 & 1 & 0.5 \\
\hline \hline
\end{tabular}

\begin{tabular}{lcccc}
\hline \hline \multicolumn{1}{c}{ Species } & $\begin{array}{c}\text { Mean \% } \\
\text { Cover }\end{array}$ & $\begin{array}{c}\text { Standard } \\
\text { Error }\end{array}$ & Presence & Prominence \\
\hline \hline CRYPFEN6 & 0.38 & 0.13 & 3 & 0.4 \\
\hline ROSAWOO5 & 0.13 & 0.13 & 1 & 0.3 \\
\hline SOLIMIS6 & 0.13 & 0.13 & 1 & 0.3 \\
\hline ARTECAM & 0.13 & 0.13 & 1 & 0.3 \\
\hline SALSKAL6 & 0.13 & 0.13 & 1 & 0.3 \\
\hline EQUIHYE6 & 0.13 & 0.13 & 1 & 0.3 \\
\hline CHENSUB6 & 0.13 & 0.13 & 1 & 0.3 \\
\hline DESCSOP6 & 0.13 & 0.13 & 1 & 0.3 \\
\hline ARTEFRI6 & 0.13 & 0.13 & 1 & 0.3 \\
\hline OPUNPOL6 & 0.13 & 0.13 & 1 & 0.3 \\
\hline FRANACA6 & 0.13 & 0.13 & 1 & 0.3 \\
\hline ELYMCAN7 & 0.13 & 0.13 & 1 & 0.3 \\
\hline \hline DECAY9 & 3.88 & 3.71 & & \\
\hline MINERAL9 & 46.25 & 5.54 & & \\
\hline ORG9 & 6.00 & 2.31 & & \\
\hline \hline
\end{tabular}

This community was consistently found on upper slope or crest topographic positions on dunes, with very rapid to rapid drainage. Slopes were strong, ranging from a minimum of $25 \%$ to $75 \%$ and generally had a south to southwesterly aspect. Little organic matter was present on the soil surface, typically with up to $40 \%$ exposed sand.

A summary of site data is provided in Table 18.

Table 18. Summary of site data for the Prunus virginiana / Calamovilfa longifolia community type $(n=4)$.

\begin{tabular}{ccccccccc}
\hline \hline Plot Elevation & Aspect & Slope & Drainage & Site Position & Surface Shape & $\begin{array}{c}\text { Moisture } \\
\text { Regime }\end{array}$ & Nutrient Regime \\
\hline \hline 10 & 874 & 228 & 55 & Very Rapid & Crest & Straight & Xeric & Submesotrophic \\
\hline 13 & 876 & 239 & 75 & Very Rapid & Upper Slope & Straight & Xeric & Submesotrophic \\
\hline 14 & 876 & 6 & 25 & Rapid & Upper Slope & Straight & Xeric & Submesotrophic \\
\hline 34 & 891 & 199 & 25 & Rapid & Crest & Convex & Xeric & Submesotrophic \\
\hline \hline
\end{tabular}




\subsubsection{Eurotia lanata / Stipa comata - Calamovilfa longifolia Association}

Winter fat / needle and thread - sand grass

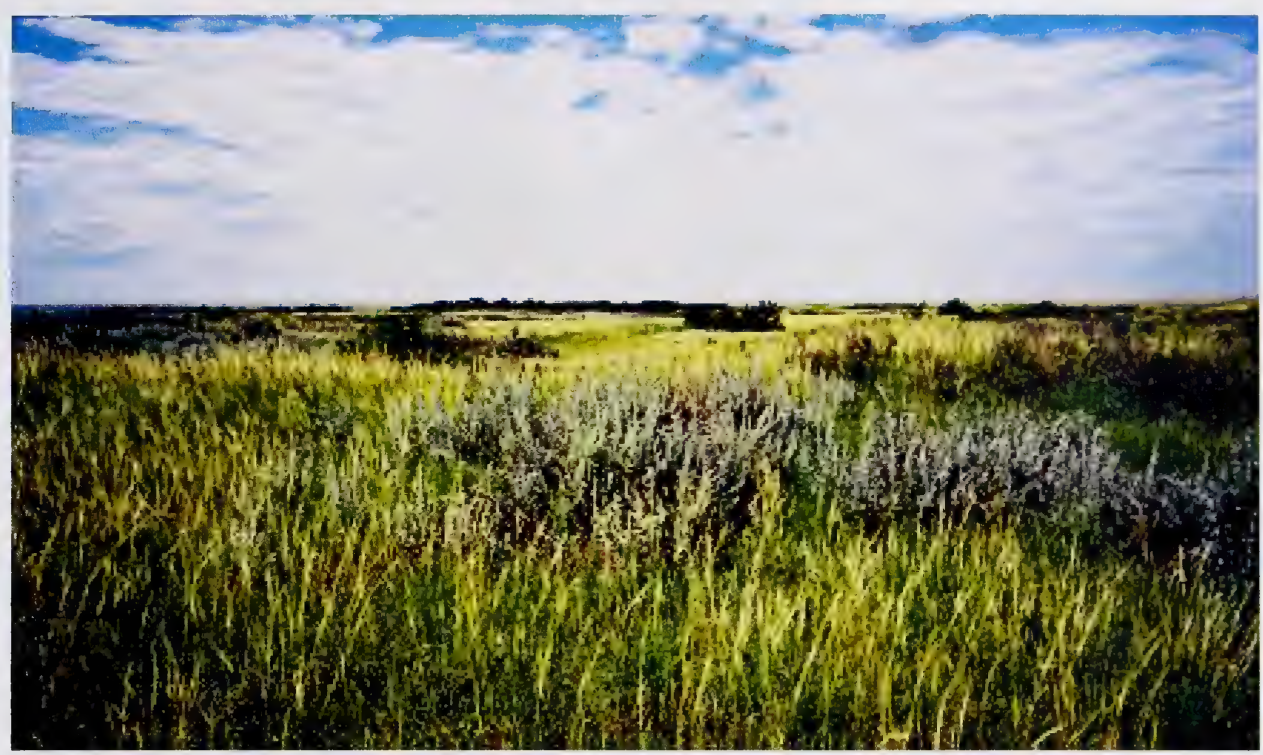

Plate 7. Eurotia lanata / Stipa comata - Calamovilfa longifolia community type (Winterfat / needle and thread - sand grass) Plot 40.

This community type was dominated by Stipa comata with a low shrub layer composed of Eurotia lanata and Rosa woodsii. Stipa comata constituted 60 \% cover, with Eurotia lanata providing $20 \%$ cover. Calamovilfa longifolia contributed approximately $15 \%$ cover. Forbs provided less than 10\% cover and typical forbs included Opuntia polycantha, Chenopodium pratericola, Artemisia frigida and Heterotheca villosa. The community was extensive with localized dense clumps of Eurotia lanata scattered throughout the Stipa comata, which formed pure localized grasslands. Table 19 summarizes the species composition and mean percent cover for this community type. 
Table 19. Summary statistics for the Eurotia lanata / Stipa comata community type $(n=1)$.

\begin{tabular}{lc}
\hline \hline \multicolumn{1}{c}{ Species } & $\begin{array}{c}\text { Percent } \\
\text { Cover }\end{array}$ \\
\hline \hline STIPCOM7 & 60 \\
\hline EUROLAN5 & 20 \\
\hline CALALON7 & 15 \\
\hline ROSAWOO5 & 5 \\
\hline OPUNPOL6 & 2 \\
\hline CHENPRA6 & 1 \\
\hline ARTEFRI6 & 1 \\
\hline HETEVIL6 & 1 \\
\hline EQUIHYE6 & 0.5 \\
\hline ARTELUD6 & 0.5 \\
\hline CRYPFEN6 & 0.5 \\
\hline CHENSUB6 & 0.5 \\
\hline \hline MINERAL9 & 10 \\
\hline ORG9 & 40 \\
\hline \hline
\end{tabular}

This community was found mid-slope on an elevated dune flank, which graded into a level plain to the east. The soils were rapidly drained with a straight surface shape. A summary of site data is provided in Table 20.

Table 20. Summary of site data for the Eurotia lanata / Stipa comata community type $(n=1)$.

\begin{tabular}{ccccccccc}
\hline \hline Plot & Elevation & Aspect & Slope & Drainage & Site Position & $\begin{array}{c}\text { Surface } \\
\text { Shape }\end{array}$ & $\begin{array}{c}\text { Moisture } \\
\text { Regime }\end{array}$ & Nutrient Regime \\
\hline \hline 40 & 866 & $\mathrm{n} / \mathrm{a}$ & 0 & Rapid & Level & Straight & Xeric & Submesotrophic \\
\hline \hline
\end{tabular}




\subsubsection{Salix exigua / Glycyrrhiza lepidota - Juncus balticus Association}

Sandbar willow / wild licorice - wire rush

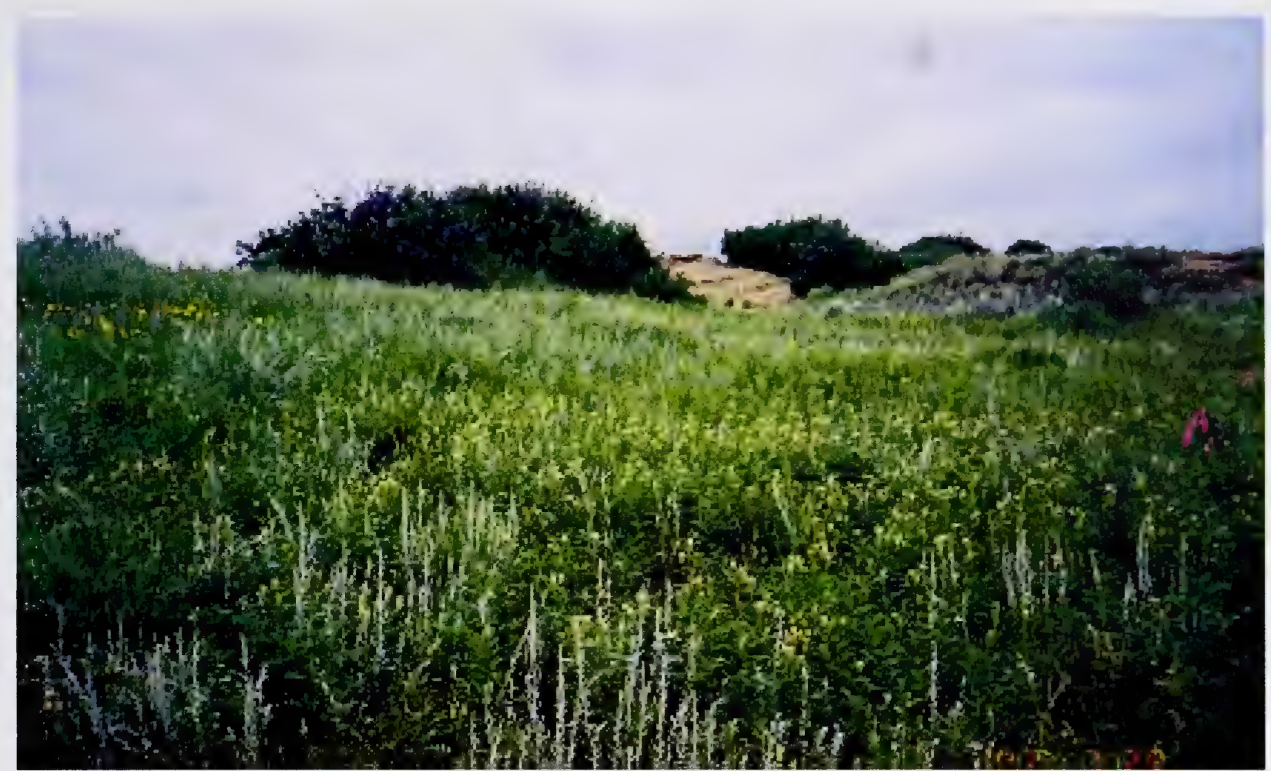

Plate 8. Salix exigua / Glycyrrhiza lepidota - Juncus balticus community type (Sandbar willow / wild licorice - wire rush) Plot 7.

This community type was co-dominated by Glycyrrhiza lepidota in the forb layer and Juncus balticus in the graminoid layer. Salix exigua was the dominant shrub, with a cover ranging from 7 to $20 \%$. Other prevalent species included Carex lanuginosa and Poa pratensis in the graminoid layer and Solidago missouriensis in the forb layer. However, these species each typically had a cover of less than $3 \%$. Table 21 summarizes the species composition, mean percent cover and standard error and species prominence for this community type. 
Table 21. Summary statistics for the Salix exigua / Glycyrrhiza lepidota - Juncus balticus community type $(\mathrm{n}=2)$.

\begin{tabular}{lcccc}
\hline Species & $\begin{array}{c}\text { Mean \% } \\
\text { Cover }\end{array}$ & $\begin{array}{c}\text { Standard } \\
\text { Error }\end{array}$ & Presence & Prominence \\
\hline \hline GLYCLEP6 & 45.00 & 5.00 & 2 & 45.0 \\
\hline JUNCBAL7 & 42.50 & 17.50 & 2 & 42.5 \\
\hline SALIEXI5 & 13.50 & 6.50 & 2 & 13.5 \\
\hline ARTEFRI6 & 2.50 & 2.50 & 1 & 3.5 \\
\hline SOLIMIS6 & 1.50 & 0.50 & 2 & 1.5 \\
\hline SALIAMY5 & 1.00 & 1.00 & 1 & 1.4 \\
\hline CHENSUB6 & 1.00 & 1.00 & 1 & 1.4 \\
\hline HELIANN6 & 1.00 & 1.00 & 1 & 1.4 \\
\hline CARELAN7 & 0.75 & 0.25 & 2 & 0.8 \\
\hline POAPRA7 & 0.75 & 0.25 & 2 & 0.8 \\
\hline SOLICAN6 & 0.50 & 0.50 & 1 & 0.7 \\
\hline DESCSOP6 & 0.25 & 0.25 & 1 & 0.4 \\
\hline \hline
\end{tabular}

\begin{tabular}{lcccc}
\hline \hline \multicolumn{1}{c}{ Species } & $\begin{array}{c}\text { Mean \% } \\
\text { Cover }\end{array}$ & $\begin{array}{c}\text { Standard } \\
\text { Error }\end{array}$ & Presence & Prominence \\
\hline \hline SMILSTE6 & 0.25 & 0.25 & 1 & 0.4 \\
\hline CHENFRE6 & 0.25 & 0.25 & 1 & 0.4 \\
\hline OENONUT6 & 0.25 & 0.25 & 1 & 0.4 \\
\hline HETEVIL6 & 0.25 & 0.25 & 1 & 0.4 \\
\hline ANTIMIC6 & 0.25 & 0.25 & 1 & 0.4 \\
\hline LATUPUL6 & 0.25 & 0.25 & 1 & 0.4 \\
\hline AGRODAS7 & 0.25 & 0.25 & 1 & 0.4 \\
\hline ELYMCAN7 & 0.25 & 0.25 & 1 & 0.4 \\
\hline SPORCRY7 & 0.25 & 0.25 & 1 & 0.4 \\
\hline CALALON7 & 0.25 & 0.25 & 1 & 0.4 \\
\hline \hline MINERAL9 & 2.00 & 0.00 & & \\
\hline \hline
\end{tabular}

This community type was most commonly found in concave, well-drained depressions between sand dunes. The soil moisture regime ranged from subxeric to submesic. A summary of site data is provided in Table 22 .

Table 22. Summary of site data for the Salix exigua / Glycyrrhiza lepidota - Juncus balticus community type $(\mathrm{n}=2)$.

\begin{tabular}{ccccccccc}
\hline \hline Plot & Elevation & Aspect & Slope & Drainage & Site Position & $\begin{array}{c}\text { Surface } \\
\text { Shape }\end{array}$ & $\begin{array}{c}\text { Moisture } \\
\text { Regime }\end{array}$ & Nutrient Regime \\
\hline \hline 2 & 879 & $\mathrm{n} / \mathrm{a}$ & 0 & Well & Depression & Concave & Submesic & Submesotrophic \\
\hline 7 & 862 & $\mathrm{n} / \mathrm{a}$ & 0 & Well & Depression & Concave & Subxeric & Mesotrophic \\
\hline \hline
\end{tabular}




\subsubsection{Glycyrrhiza lepidota - Calamovilfa longifolia Association}

Wild licorice - sand grass

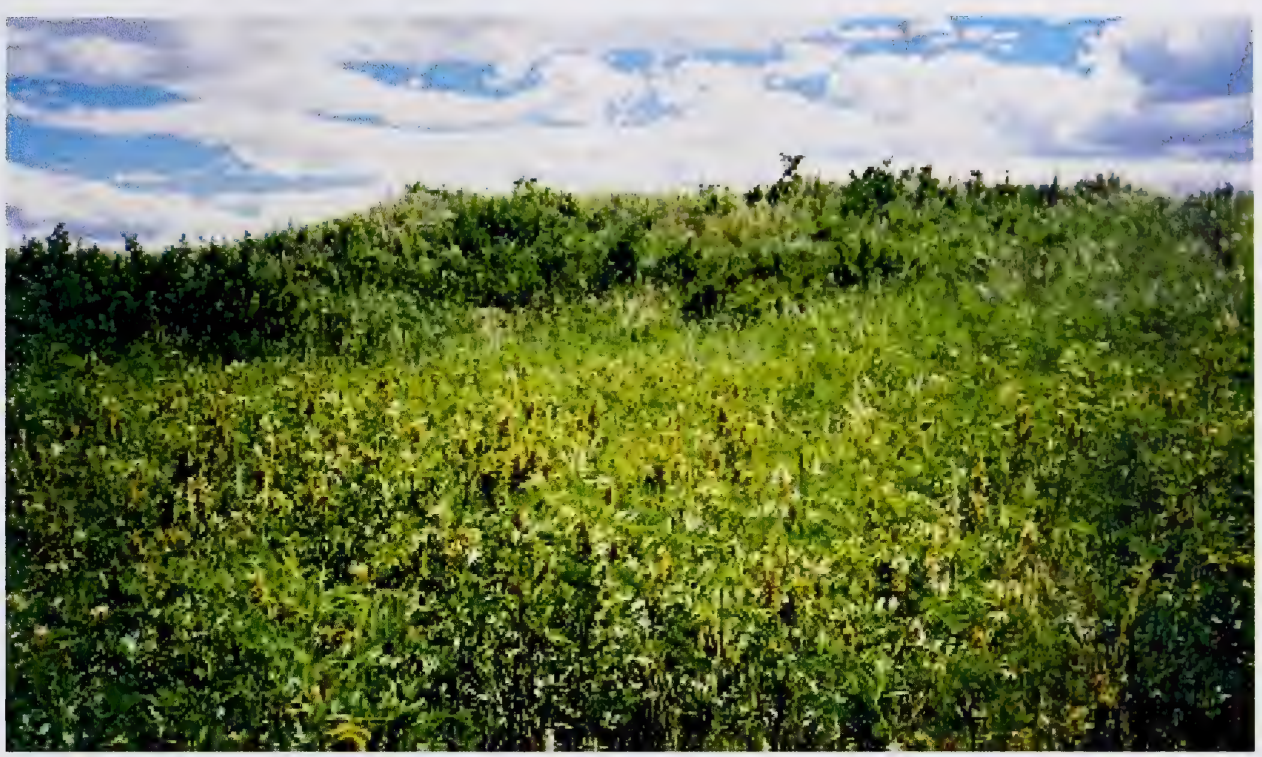

Plate 9. Glycyrrhiza lepidota - Calamovilfa longifolia community type (Wild licorice sand grass) Plot 36.

This community type was dominated by Glycyrrhiza lepidota in the forb stratum and Calamovilfa longifolia in the grass stratum. Where this community was surveyed, Glycyrrhiza lepidota had a cover $60 \%$. A sparse shrub layer was present, which included Prunus virginiana and Rosa woodsii with a cover of less than $10 \%$. Several forbs were present including Artemisia ludoviciana, A. frigida and Chenopodium pratericola, among others. Stipa comata, Juncus balticus and Agropyron dasystachyum were also present, but with a total cover of less than $10 \%$. Table 23 summarizes the species composition and percent cover for this community type. 
Table 23. Summary statistics for the Glycyrrhiza lepidota-Calamovilfa longifolia community type $(\mathrm{n}=1)$.

\begin{tabular}{lc}
\hline Species & $\begin{array}{c}\text { Percent } \\
\text { Cover }\end{array}$ \\
\hline \hline GLYCLEP6 & 60 \\
\hline CALALON7 & 25 \\
\hline STIPCOM7 & 7 \\
\hline PRUNVIR5 & 5 \\
\hline ARTELUD6 & 2 \\
\hline ROSAWOO5 & 1 \\
\hline CHENPRA6 & 1 \\
\hline ARTEFRI6 & 1 \\
\hline HETEVIL6 & 1 \\
\hline JUNCBAL7 & 1 \\
\hline \hline
\end{tabular}

\begin{tabular}{lc}
\hline Species & $\begin{array}{c}\text { Percent } \\
\text { Cover }\end{array}$ \\
\hline \hline CORYVIV6 & 0.5 \\
\hline EQUIHYE6 & 0.5 \\
\hline CRYPFEN6 & 0.5 \\
\hline CHENSUB6 & 0.5 \\
\hline DESCSOP6 & 0.5 \\
\hline CHENFRE6 & 0.5 \\
\hline OPUNPOL6 & 0.5 \\
\hline CERAARV6 & 0.5 \\
\hline AGRODAS7 & 0.5 \\
\hline \hline MINERAL9 & 5 \\
\hline ORG9 & 10 \\
\hline \hline
\end{tabular}

This community type was found in a small inter-dune depression with well-drained soils. A summary of site data is provided in Table 24.

Table 24. Summary of site data for the Glycyrrhiza lepidota - Calamovilfa longifolia community type $(\mathrm{n}=1)$.

\begin{tabular}{lcccccccc}
\hline \hline Plot & Elevation & Aspect & Slope & Drainage & Site Position & $\begin{array}{c}\text { Surface } \\
\text { Shape }\end{array}$ & $\begin{array}{c}\text { Moisture } \\
\text { Regime }\end{array}$ & Nutrient Regime \\
\hline \hline 36 & 893 & $\mathrm{n} / \mathrm{a}$ & 0 & Well & Depression & Concave & Subxeric & Submesotrophic \\
\hline \hline
\end{tabular}




\subsubsection{Glycyrrhiza lepidota - Artemisia spp. - Stipa comata Association} Wild licorice - sage - needle and thread

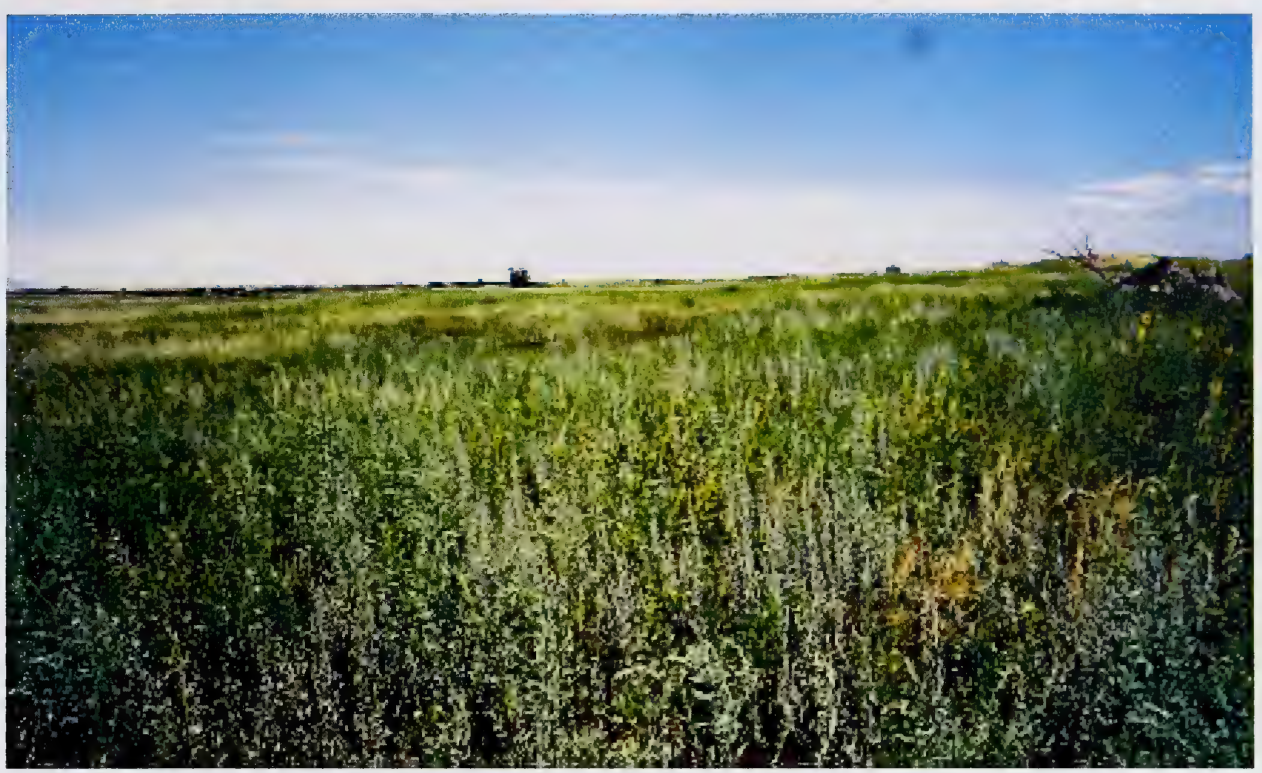

Plate 10. Glycyrrhiza lepidota - Artemisia spp. - Stipa comata community type (Wild licorice - sage - needle and thread) Plot 19.

This community type was co-dominated by Glycyrrhiza lepidota in the forb stratum and Stipa comata in the graminoid stratum. Several species of Artemisia were also common, including Artemisia ludoviciana and A. frigida. Other common species with lower percent covers included Heterotheca villosa, Chenopodium pratericola and Tragopogon dubius in the forb layer and Juncus balticus in the graminoid layer. Table 25 summarizes the species composition, mean percent cover, standard error and species prominence for this community type. 
Table 25. Summary statistics for the Glycyrrhiza lepidota - Artemisia spp. - Stipa comata community type $(\mathrm{n}=2)$.

\begin{tabular}{lcccc}
\hline \hline Species & $\begin{array}{c}\text { Mean \% } \\
\text { Cover }\end{array}$ & $\begin{array}{c}\text { Standard } \\
\text { Error }\end{array}$ & Presence & Prominence \\
\hline \hline GLYCLEP6 & 32.50 & 7.50 & 2 & 32.5 \\
\hline STIPCOM7 & 25.00 & 15.00 & 2 & 25.0 \\
\hline ARTELUD6 & 13.00 & 12.00 & 2 & 13.0 \\
\hline JUNCBAL7 & 5.00 & 5.00 & 1 & 7.1 \\
\hline HETEVIL6 & 2.50 & 2.50 & 1 & 3.5 \\
\hline ARTEFRI6 & 3.00 & 2.00 & 2 & 3.0 \\
\hline CHENPRA6 & 1.50 & 0.50 & 2 & 1.5 \\
\hline ROSAWOO5 & 1.00 & 1.00 & 1 & 1.4 \\
\hline TRAGDUB6 & 0.75 & 0.25 & 2 & 0.8 \\
\hline KOELMAC7 & 0.50 & 0.50 & 1 & 0.7 \\
\hline \hline
\end{tabular}

\begin{tabular}{lcccc}
\hline \hline Species & $\begin{array}{c}\text { Mean \% } \\
\text { Cover }\end{array}$ & $\begin{array}{c}\text { Standard } \\
\text { Error }\end{array}$ & Presence & Prominence \\
\hline \hline AGROSIB7 & 0.50 & 0.50 & 1 & 0.7 \\
\hline POAPRA7 & 0.50 & 0.50 & 1 & 0.7 \\
\hline ACHIMIL6 & 0.25 & 0.25 & 1 & 0.4 \\
\hline CRYPFEN6 & 0.25 & 0.25 & 1 & 0.4 \\
\hline CHENSUB6 & 0.25 & 0.25 & 1 & 0.4 \\
\hline DESCSOP6 & 0.25 & 0.25 & 1 & 0.4 \\
\hline HELIANN6 & 0.25 & 0.25 & 1 & 0.4 \\
\hline AGRODAS7 & 0.25 & 0.25 & 1 & 0.4 \\
\hline \hline MINERAL9 & 12.50 & 2.50 & & \\
\hline ORG9 & 30.00 & 0.00 & & \\
\hline \hline
\end{tabular}

This plant community was typically found in level, very rapidly drained sand plain habitats. Organic matter accumulation at the soil surface averaged $30 \%$ cover. A summary of site data is provided in Table 26.

Table 26. Summary of site data for the Glycyrrhiza lepidota - Artemisia spp. - Stipa comata community type $(n=2)$.

\begin{tabular}{lcccccccl}
\hline Plot Elevation & Aspect & Slope & Drainage & Site Position & $\begin{array}{c}\text { Surface } \\
\text { Shape }\end{array}$ & $\begin{array}{c}\text { Moisture } \\
\text { Regime }\end{array}$ & Nutrient Regime \\
\hline \hline 19 & 866 & n/a & 0 & Very Rapid & Level & Straight & Xeric & Submesotrophic \\
\hline 20 & 865 & n/a & 0 & Very Rapid & Level & Straight & Xeric & Submesotrophic \\
\hline \hline
\end{tabular}




\subsubsection{Rumex venosus Association}

Wild begonia

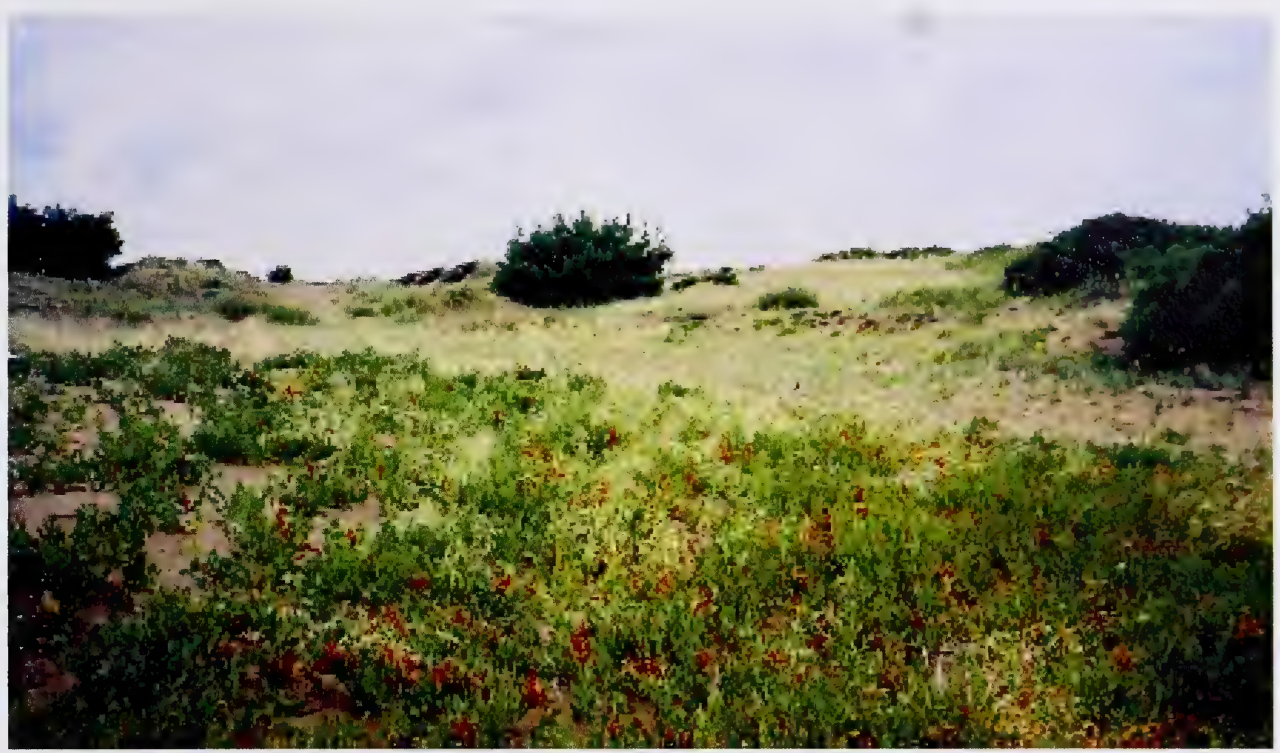

Plate 11. Rumex venosus community type (Wild begonia) Plot 6.

This community type was dominated by Rumex venosus in the forb stratum. It was typically found on the leeward side of active sand dunes, where sand accumulation was occurring. There was little grass cover, although Sporobolus cryptandrus was found in both plots (under $2 \%$ cover) and at one site, Oryzopsis hymenoides provided $5 \%$ cover. Table 27 summarizes the species composition, mean percent cover, standard error and species prominence for this community type.

Table 27. Summary statistics for the Rumex venosus community type $(\mathrm{n}=2)$.

\begin{tabular}{lcccc}
\hline \hline Species & $\begin{array}{c}\text { Mean \% } \\
\text { Cover }\end{array}$ & $\begin{array}{c}\text { Standard } \\
\text { Error }\end{array}$ & Presence & Prominence \\
\hline \hline RUMEVEN6 & 42.50 & 17.50 & 2 & 42.5 \\
\hline ORYZHYM7 & 2.50 & 2.50 & 1 & 3.5 \\
\hline FRANACA6 & 1.00 & 1.00 & 1 & 1.4 \\
\hline HELIANN6 & 1.00 & 1.00 & 1 & 1.4 \\
\hline SPORCRY7 & 1.25 & 0.75 & 2 & 1.3 \\
\hline THERRHO6 & 0.50 & 0.50 & 1 & 0.7 \\
\hline ROSAWOO5 & 0.25 & 0.25 & 1 & 0.4 \\
\hline HETEVIL6 & 0.25 & 0.25 & 1 & 0.4 \\
\hline ELYMCAN7 & 0.25 & 0.25 & 1 & 0.4 \\
\hline CALALON7 & 0.25 & 0.25 & 1 & 0.4 \\
\hline \hline MINERAL9 & 55.00 & 15.00 & & \\
\hline ORG9 & 0.50 & 0.00 & & \\
\hline \hline
\end{tabular}


This community type was typically found on the leeward side of dunes with slopes ranging from $18-30 \%$ and a north to northeast aspect. Sites were located on the mid to upper slopes of dunes and were very rapidly to rapidly drained. Exposed mineral soil comprised 40 to $70 \%$ cover, with virtually no organic matter accumulation at the soil surface. A summary of site data is provided in Table 28.

Table 28. Summary of site data for the Rumex venosus community type $(\mathrm{n}=2)$.

\begin{tabular}{ccccccccc}
\hline Plot & Elevation & Aspect & Slope & Drainage & Site Position & $\begin{array}{c}\text { Surface } \\
\text { Shape }\end{array}$ & $\begin{array}{c}\text { Moisture } \\
\text { Regime }\end{array}$ & Nutrient Regime \\
\hline \hline 6 & 863 & 37 & 18 & Rapid & Middle Slope & Straight & Xeric & Submesotrophic \\
\hline 30 & 887 & 48 & 30 & Very Rapid & Upper Slope & Straight & Xeric & Submesotrophic \\
\hline \hline
\end{tabular}




\subsubsection{Oryzopsis hymenoides - Sporobolus cryptandrus Association} Indian rice grass - sand dropseed

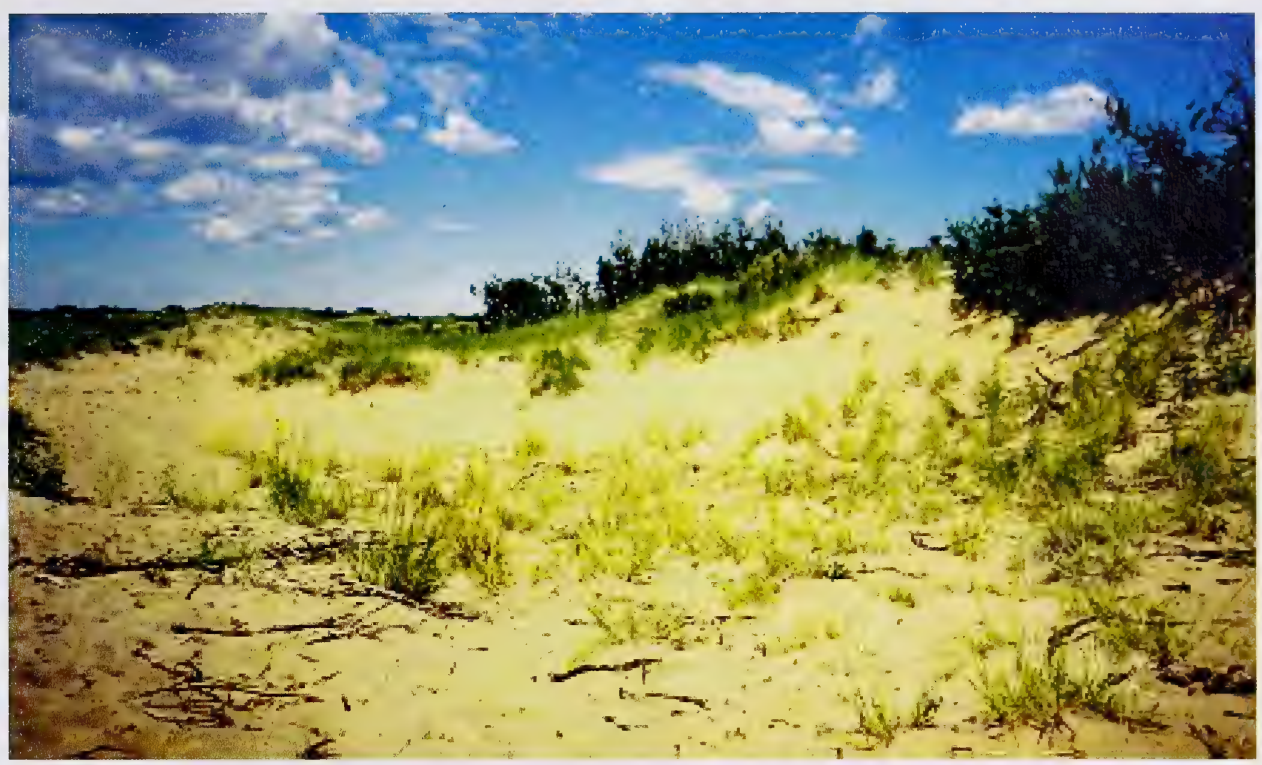

Plate 12. Oryzopsis hymenoides - Sporobolus cryptandrus community type (Indian rice grass - sand dropseed) Plot 35 .

This community was co-dominated by Oryzopsis hymenoides and Sporobolus cryptandrus in the graminoid stratum, however, total percent cover was generally less than $40 \%$. This community was typically found on dune sites, where sand movement was active. As such, only one shrub species (Elaeagnus commutata) was encountered with less than $1 \%$ cover. Two forb species were common to all or most survey sites, including Helianthus annuus and Heterotheca villosa. Other forb and graminoid species were found at different survey sites, but few were common to all or most plots. Table 29 summarizes the species composition, mean percent cover and standard error and species prominence for this community type. 
Table 29. Summary statistics for the Oryzopsis hymenoides - Sporobolus cryptandrus community type $(\mathrm{n}=5)$.

\begin{tabular}{lcccc}
\hline \hline Species & $\begin{array}{c}\text { Mean \% } \\
\text { Cover }\end{array}$ & $\begin{array}{c}\text { Standard } \\
\text { Error }\end{array}$ & Presence & Prominence \\
\hline \hline SPORCRY7 & 17.20 & 5.69 & 5 & 17.2 \\
\hline ORYZHYM7 & 10.00 & 2.24 & 5 & 10.0 \\
\hline THERRHO6 & 4.40 & 3.92 & 2 & 7.0 \\
\hline HELIANN6 & 2.80 & 0.92 & 5 & 2.8 \\
\hline RUMEVEN6 & 1.30 & 0.94 & 3 & 1.7 \\
\hline HETEVIL6 & 0.70 & 0.20 & 4 & 0.8 \\
\hline CALALON7 & 0.30 & 0.20 & 2 & 0.5 \\
\hline SOLIMIS6 & 0.20 & 0.20 & 1 & 0.4 \\
\hline LYGOROS6 & 0.20 & 0.20 & 1 & 0.4 \\
\hline FRANACA6 & 0.20 & 0.20 & 1 & 0.4 \\
\hline \hline
\end{tabular}

\begin{tabular}{lcccc}
\hline \multicolumn{1}{c}{ Species } & $\begin{array}{c}\text { Mean \% } \\
\text { Cover }\end{array}$ & $\begin{array}{c}\text { Standard } \\
\text { Error }\end{array}$ & Presence & Prominence \\
\hline \hline GLYCLEP6 & 0.20 & 0.20 & 1 & 0.4 \\
\hline CYPESCH7 & 0.20 & 0.20 & 1 & 0.4 \\
\hline KOELMAC7 & 0.20 & 0.20 & 1 & 0.4 \\
\hline ELYMCAN7 & 0.20 & 0.20 & 1 & 0.4 \\
\hline CHENPRA6 & 0.20 & 0.12 & 2 & 0.3 \\
\hline ELAECOM5 & 0.10 & 0.10 & 1 & 0.2 \\
\hline AGRODAS7 & 0.10 & 0.10 & 1 & 0.2 \\
\hline \hline DECAY9 & 2.00 & 2.00 & & \\
\hline MINERAL9 & 62.00 & 12.71 & & \\
\hline ORG9 & 3.80 & 2.81 & & \\
\hline \hline
\end{tabular}

This community type was commonly found on south to southwest facing slopes of active dunes. Sand movement was evident and in most sites the percentage of exposed sand at the soil surface exceeded $60 \%$ (reaching up to $90 \%$ ). There was little organic matter accumulation at the soil surface and some decaying wood (shrub branches) was found at one survey site. A summary of site data is provided in Table 30.

Table 30. Summary of site data for the Oryzopsis hymenoides - Sporobolus cryptandrus community type $(n=5)$.

\begin{tabular}{ccccccccc}
\hline \hline Plot & Elevation & Aspect & Slope & Drainage & Site Position & $\begin{array}{c}\text { Surface } \\
\text { Shape }\end{array}$ & $\begin{array}{c}\text { Moisture } \\
\text { Regime }\end{array}$ & Nutrient Regime \\
\hline \hline 21 & 862 & 168 & 15 & Very Rapid & Upper Slope & Straight & Very Xeric & Oligotrophic \\
\hline 29 & 888 & 11 & 25 & Very Rapid & Middle Slope & Straight & Xeric & Oligotrophic \\
\hline 31 & 894 & 284 & 25 & Rapid & Upper Slope & Straight & Xeric & Submesotrophic \\
\hline 35 & 891 & n/a & 0 & Rapid & Upper Slope & Concave & Xeric & Submesotrophic \\
\hline 37 & 886 & 207 & 10 & Very Rapid & Lower Slope & Concave & Very Xeric & Oligotrophic \\
\hline \hline
\end{tabular}




\subsubsection{Stipa comata - Oryzopsis hymenoides Association}

Needle and thread - Indian rice grass

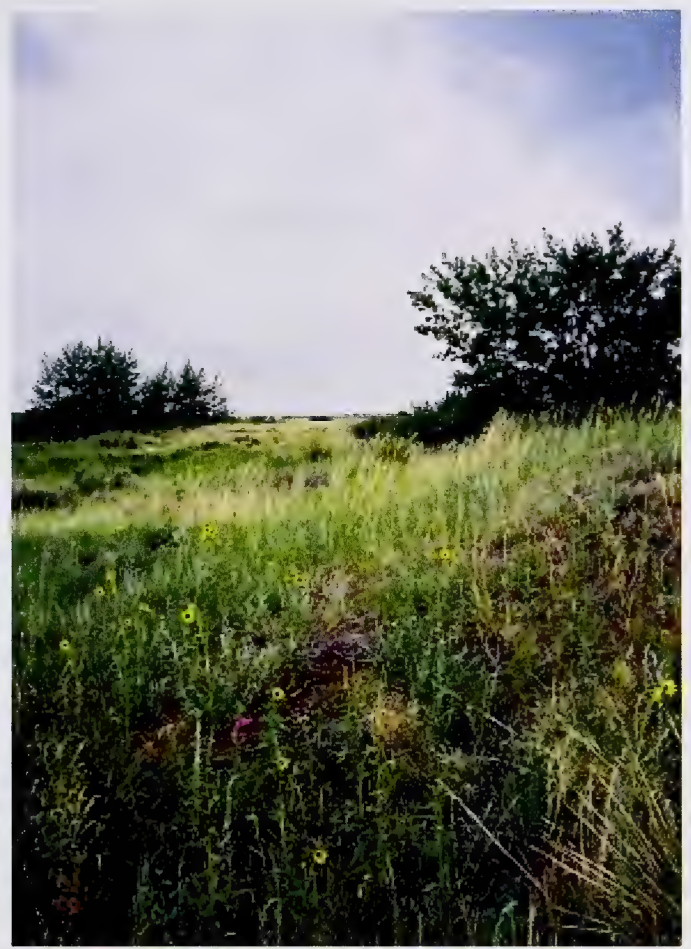

Plate 13. Stipa comata - Oryzopsis hymenoides community type (Needle and thread Indian rice grass) Plot 8.

This community was co-dominated by Stipa comata and Oryzopsis hymenoides, although their combined cover was typically less than $20 \%$. This community was found on partially stabilized dunes. Shrub species, including Elaeagnus commutata, Rosa woodsii and Salix exigua were encountered though with very low percent covers (generally less than $2 \%$ ). Forb species that were relatively common (at low percent cover) included Helianthus annuus, Chenopodium pratericola, C. subglabrum and Lygodesmia rostrata. Table 31 summarizes the species composition, mean percent cover, standard error and species prominence for this community type. 
Table 31. Summary statistics for the Stipa comata-Oryzopsis hymenoides community type $(n=2)$.

\begin{tabular}{lcccc}
\hline \hline Species & $\begin{array}{c}\text { Mean \% } \\
\text { Cover }\end{array}$ & $\begin{array}{c}\text { Standard } \\
\text { Error }\end{array}$ & Presence & Prominence \\
\hline \hline ORYZHYM7 & 6.50 & 3.50 & 2 & 6.5 \\
\hline STIPCOM7 & 5.00 & 0.00 & 2 & 5.0 \\
\hline CRYPFEN6 & 2.50 & 2.50 & 1 & 3.5 \\
\hline SPORCRY7 & 2.50 & 2.50 & 1 & 3.5 \\
\hline HELIANN6 & 2.50 & 0.50 & 2 & 2.5 \\
\hline THERRHO6 & 1.50 & 1.50 & 1 & 2.1 \\
\hline AGROSMI7 & 1.50 & 1.50 & 1 & 2.1 \\
\hline CHENSUB6 & 0.75 & 0.25 & 2 & 0.8 \\
\hline CHENPRA6 & 1.50 & 0.50 & 2 & 1.5 \\
\hline LYGOROS6 & 0.75 & 0.25 & 2 & 0.8 \\
\hline \hline
\end{tabular}

\begin{tabular}{lcccc}
\hline \hline \multicolumn{1}{c}{ Species } & $\begin{array}{c}\text { Mean \% } \\
\text { Cover }\end{array}$ & $\begin{array}{c}\text { Standard } \\
\text { Error }\end{array}$ & Presence & Prominence \\
\hline \hline ROSAWOO5 & 0.50 & 0.50 & 1 & 0.7 \\
\hline HETEVIL6 & 0.50 & 0.50 & 1 & 0.7 \\
\hline GLYCLEP6 & 0.50 & 0.50 & 1 & 0.7 \\
\hline ELAECOM5 & 0.25 & 0.25 & 1 & 0.4 \\
\hline SALIEXI5 & 0.25 & 0.25 & 1 & 0.4 \\
\hline RUMEVEN6 & 0.25 & 0.25 & 1 & 0.4 \\
\hline KOELMAC7 & 0.25 & 0.25 & 1 & 0.4 \\
\hline \hline MINERAL9 & 22.50 & 12.50 & & \\
\hline ORG9 & 7.50 & 2.50 & & \\
\hline \hline
\end{tabular}

This community was typically found on mid to upper slope positions of moderately inclined south to southeast facing slopes. Exposed sand at the soil surface ranged from 10 to $35 \%$ and organic matter accumulation was typically less than $10 \%$ cover. A summary of site data is provided in Table 32.

Table 32. Summary of site data for the Stipa comata-Oryzopsis hymenoides community type $(n=2)$.

\begin{tabular}{ccccccccc}
\hline \hline Plot & Elevation & Aspect & Slope & Drainage & Site Position & $\begin{array}{c}\text { Surface } \\
\text { Shape }\end{array}$ & $\begin{array}{c}\text { Moisture } \\
\text { Regime }\end{array}$ & Nutrient Regime \\
\hline \hline 8 & 868 & 162 & 15 & Rapid & Upper Slope & Straight & Xeric & Submesotrophic \\
\hline 25 & 885 & 149 & 20 & Rapid & Middle Slope & Concave & Xeric & Submesotrophic \\
\hline \hline
\end{tabular}




\subsubsection{Artemisia spp. - Stipa comata - Calamovilfa longifolia Association} Sage - needle and thread - sand grass

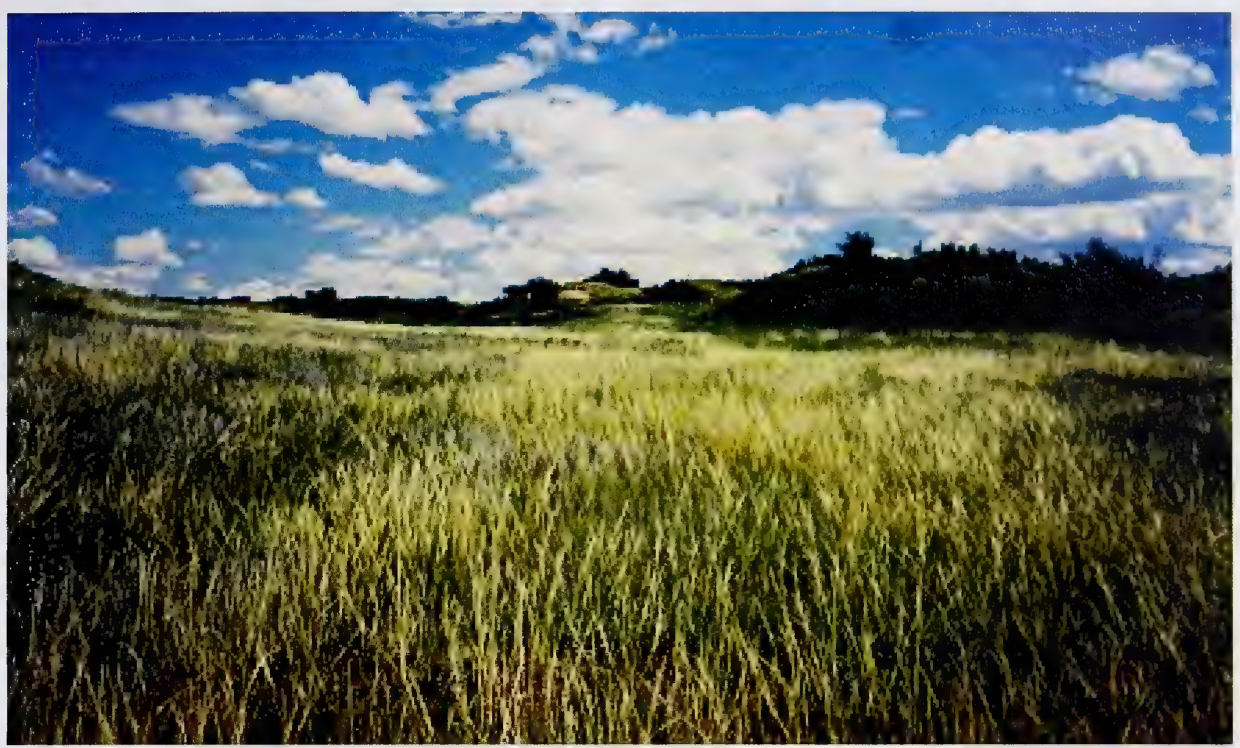

Plate 14. Artemisia spp. - Stipa comata - Calamovilfa longifolia (Sage - needle and thread - sand grass) Plot 39.

This community was dominated by Stipa comata in the graminoid stratum and a variety of Artemisia species in the forb layer. Artemisia frigida was found at all sites, while $A$. ludoviciana was found at two sites and $A$. campestris was found at one site. Several other forbs were common in this community including Heterotheca villosa, Chenopodium pratericola and C. subglabrum, all with relatively low percent covers. Rosa woodsii was common to all survey sites, with a mean cover of less than $2 \%$. Several other grasses were common, including Calamovilfa longifolia, Koeleria macrantha and Sporobolus cryptandrus but with low percent covers. Table 33 summarizes the species composition, mean percent cover and standard error and species prominence for this community type. 
Table 33. Summary statistics for the Artemisia spp. - Stipa comata-Calamovilfa longifolia community type $(\mathrm{n}=3)$.

\begin{tabular}{lcccc}
\hline Species & $\begin{array}{c}\text { Mean \% } \\
\text { Cover }\end{array}$ & $\begin{array}{c}\text { Standard } \\
\text { Error }\end{array}$ & Presence & Prominence \\
\hline STIPCOM7 & 40.00 & 5.77 & 3 & 40.0 \\
\hline ARTECAM & 3.33 & 3.33 & 1 & 5.8 \\
\hline CALALON7 & 5.33 & 2.60 & 3 & 5.3 \\
\hline ARTEFRI6 & 2.67 & 1.20 & 3 & 2.7 \\
\hline ARTELUD6 & 2.00 & 1.53 & 2 & 2.4 \\
\hline HETEVIL6 & 2.00 & 0.58 & 3 & 2.0 \\
\hline OPUNPOL6 & 1.33 & 0.88 & 2 & 1.6 \\
\hline ROSAWOO5 & 1.50 & 0.76 & 3 & 1.5 \\
\hline SOLIMIS6 & 1.00 & 0.58 & 2 & 1.2 \\
\hline THERRHO6 & 0.83 & 0.60 & 2 & 1.0 \\
\hline KOELMAC7 & 1.00 & 0.00 & 3 & 1.0 \\
\hline CHENPRA6 & 0.83 & 0.17 & 3 & 0.8 \\
\hline SPORCRY7 & 1.00 & 0.58 & 3 & 1.0 \\
\hline \hline
\end{tabular}

\begin{tabular}{lcccc}
\hline \hline Species & $\begin{array}{c}\text { Mean \% } \\
\text { Cover }\end{array}$ & $\begin{array}{c}\text { Standard } \\
\text { Error }\end{array}$ & Presence & Prominence \\
\hline \hline LYGOROS6 & 0.33 & 0.33 & 1 & 0.6 \\
\hline AGRODAS7 & 0.33 & 0.33 & 1 & 0.6 \\
\hline CHENSUB6 & 0.50 & 0.00 & 3 & 0.5 \\
\hline LEPIDEN6 & 0.33 & 0.17 & 2 & 0.4 \\
\hline ACHIMIL6 & 0.33 & 0.17 & 2 & 0.4 \\
\hline PRUNVIR5 & 0.17 & 0.17 & 1 & 0.3 \\
\hline PLANPAT6 & 0.17 & 0.17 & 1 & 0.3 \\
\hline CRYPFEN6 & 0.17 & 0.17 & 1 & 0.3 \\
\hline DESCSOP6 & 0.17 & 0.17 & 1 & 0.3 \\
\hline GLYCLEP6 & 0.17 & 0.17 & 1 & 0.3 \\
\hline ORYZHYM7 & 0.17 & 0.17 & 1 & 0.3 \\
\hline \hline MINERAL9 & 18.33 & 1.67 & & \\
\hline ORG9 & 16.83 & 8.66 & & \\
\hline \hline
\end{tabular}

This community type was found on level, sand plain habitats that were very rapidly to well drained. A summary of site data is provided in Table 34.

Table 34. Summary of site data for the Artemisia spp. - Stipa comata - Calamovilfa longifolia community type $(\mathrm{n}=3)$.

\begin{tabular}{ccccccccc}
\hline \hline Plot Elevation & Aspect & Slope & Drainage & Site Position & $\begin{array}{c}\text { Surface } \\
\text { Shape }\end{array}$ & $\begin{array}{c}\text { Moisture } \\
\text { Regime }\end{array}$ & Nutrient Regime \\
\hline \hline 22 & 866 & $\mathrm{n} / \mathrm{a}$ & 0 & Very Rapid & Level & Straight & Xeric & Submesotrophic \\
\hline 38 & 883 & $\mathrm{n} / \mathrm{a}$ & 0 & Well & Level & Straight & Xeric & Submesotrophic \\
\hline 39 & 878 & $\mathrm{n} / \mathrm{a}$ & 0 & Rapid & Level & Straight & Xeric & Submesotrophic \\
\hline \hline
\end{tabular}




\subsubsection{Stipa comata - Cyperus schweinitzii - Calamovilfa longifolia Association}

Needle and thread - sand nut-grass - sand grass

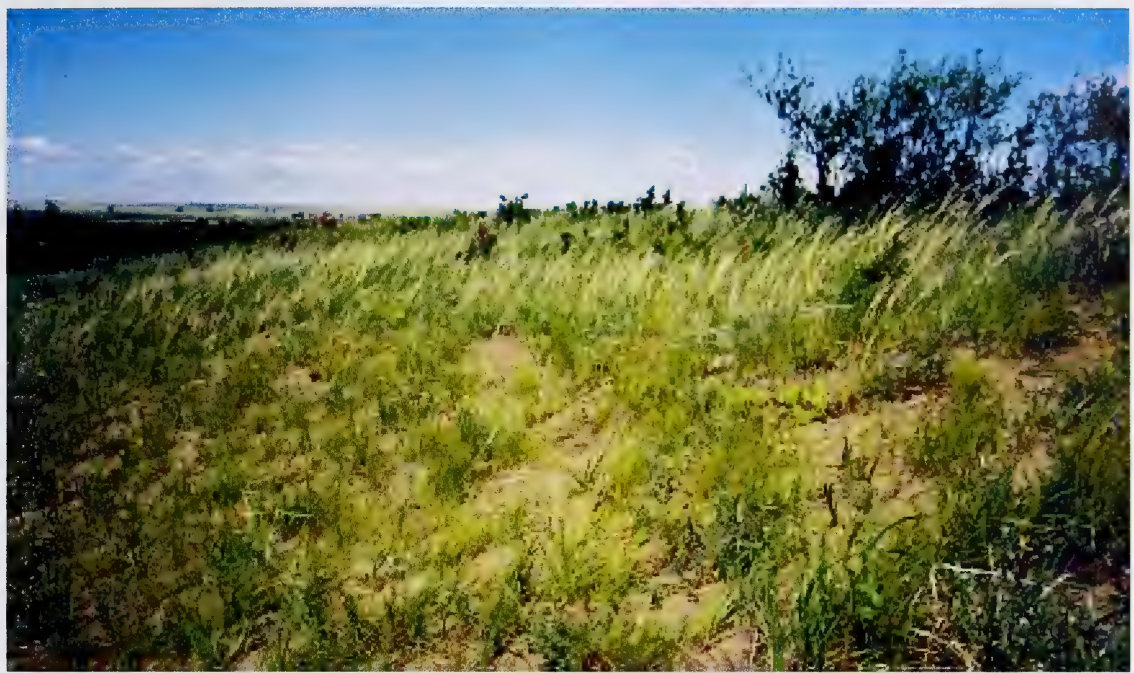

Plate 15. Stipa comata - Cyperus schweinitzii - Calamovilfa longifolia community type (Needle and thread - sand nut-grass - sand grass) Plot 33.

This community type was dominated by graminoids, with Stipa comata, Cyperus schweinitzii and Calamovilfa longifolia having the highest percent covers. Several forbs were typical, including Artemisia campestris, Lygodesmia rostrata, Opuntia polycantha and Heterotheca villosa. Rosa woodsii was also present, but with only a $1 \%$ cover. Table 35 summarizes the species composition and mean percent cover for this community type.

Table 35. Summary statistics for the Stipa comata-Cyperus schweinitzii-Calamovilfa longifolia community type $(\mathrm{n}=1)$.

\begin{tabular}{lc}
\hline Species & $\begin{array}{c}\text { Percent } \\
\text { Cover }\end{array}$ \\
\hline \hline STIPCOM7 & 25 \\
\hline CYPESCH7 & 20 \\
\hline CALALON7 & 10 \\
\hline ROSAWOO5 & 1 \\
\hline ARTECAM & 1 \\
\hline LYGOROS6 & 1 \\
\hline OPUNPOL6 & 1 \\
\hline HETEVIL6 & 1 \\
\hline CRYPFEN6 & 0.5 \\
\hline HELIANN6 & 0.5 \\
\hline SPORCRY7 & 0.5 \\
\hline \hline MINERAL9 & 70 \\
\hline ORG9 & 2 \\
\hline \hline
\end{tabular}


This site was located on a shallow, windward, south-facing dune slope. The site was very exposed to the wind and likely had active sand movement. The community had approximately $70 \%$ exposed sand at the soil surface and was very rapidly drained. A summary of site data is provided in Table 36 .

Table 36. Summary of site data for the Stipa comata - Cyperus schweinitzii Calamovilfa longifolia community type $(\mathrm{n}=1)$.

\begin{tabular}{lcccccccc}
\hline Plot Elevation & Aspect & Slope & Drainage & Site Position & $\begin{array}{c}\text { Surface } \\
\text { Shape }\end{array}$ & $\begin{array}{c}\text { Moisture } \\
\text { Regime }\end{array}$ & Nutrient Regime \\
\hline \hline 33 & 877 & 195 & 15 & Very Rapid & Upper Slope & Straight & Xeric & Submesotrophic \\
\hline \hline
\end{tabular}




\subsubsection{Sporobolus cryptandrus - Calamovilfa longifolia -Oryzopsis hymenoides Association}

Sand dropseed - sand grass - Indian rice grass

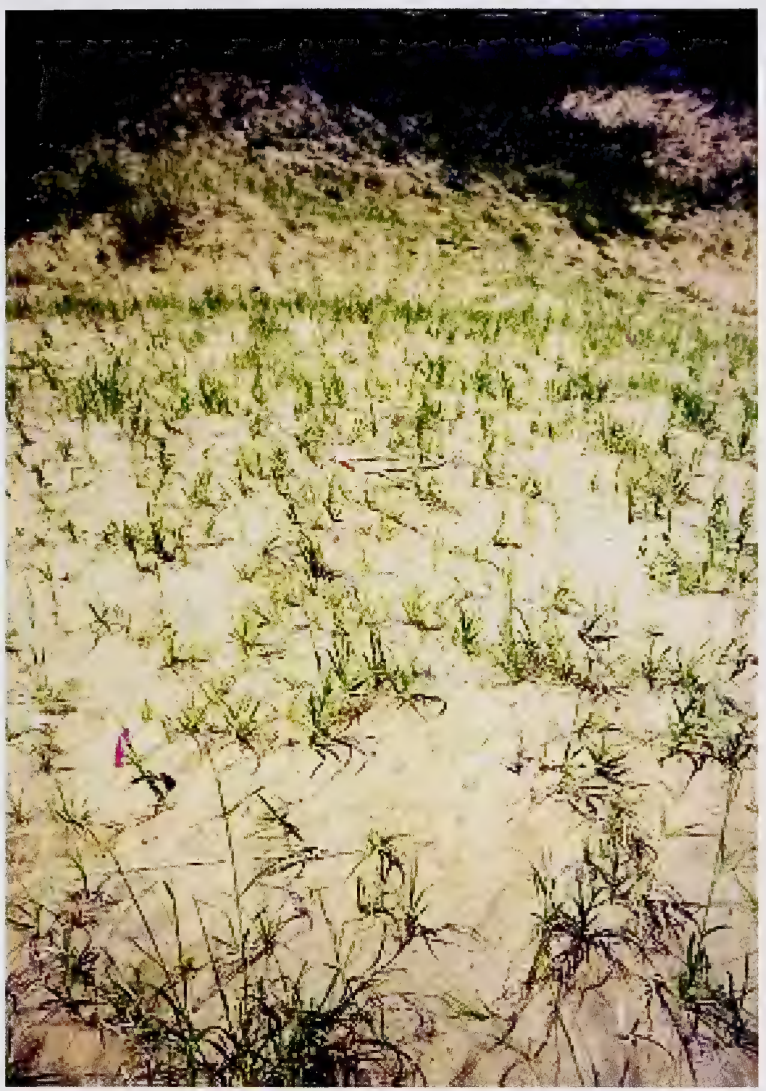

Plate 16. Sporobolus cryptandrus - Calamovilfa longifolia - Oryzopsis hymenoides community type (Sand dropseed - sand grass - Indian rice grass) Plot 4.

This community type occurred on dunes in the very early stages of stabilization, but that still have active sand movement. It was co-dominated by Sporobolus cryptandrus and Calamovilfa longifolia, with lesser amounts of Oryzopsis hymenoides. Low shrubs were present at some survey sites, however, the percent cover was quite low and included shrubs such as Eurotia lanata, Rosa woodsii and Elaeagnus commutata. A forb layer was typically present and included species such as Helianthus annuus, Lygodesmia rostrata and Heterotheca villosa. Table 37 summarizes the species composition, mean percent cover and standard error and species prominence for this community type. 
Table 37. Summary statistics for the Sporobolus cryptandrus - Calamovilfa longifoliaOryzopsis hymenoides community type $(\mathrm{n}=4)$.

\begin{tabular}{lcccc}
\hline Species & $\begin{array}{c}\text { Mean \% } \\
\text { Cover }\end{array}$ & $\begin{array}{c}\text { Standard } \\
\text { Error }\end{array}$ & Presence & Prominence \\
\hline \hline SPORCRY7 & 13.00 & 4.36 & 4 & 13.0 \\
\hline STIPCOM7 & 2.50 & 2.50 & 1 & 5.0 \\
\hline CALALON7 & 4.25 & 0.75 & 4 & 4.3 \\
\hline HELIANN6 & 3.38 & 2.30 & 3 & 3.9 \\
\hline ORYZHYM7 & 3.25 & 1.03 & 4 & 3.3 \\
\hline EUROLAN5 & 2.00 & 1.68 & 2 & 2.8 \\
\hline ROSAWOO5 & 2.00 & 1.68 & 2 & 2.8 \\
\hline ELAECOM5 & 1.25 & 1.25 & 1 & 2.5 \\
\hline GLYCLEP6 & 0.88 & 0.72 & 2 & 1.2 \\
\hline LYGOROS6 & 0.75 & 0.43 & 3 & 0.9 \\
\hline CHENSUB6 & 0.38 & 0.24 & 2 & 0.5 \\
\hline CHENPRA6 & 0.38 & 0.24 & 2 & 0.5 \\
\hline \hline
\end{tabular}

\begin{tabular}{lcccc}
\hline \multicolumn{1}{c}{ Species } & $\begin{array}{c}\text { Mean \% } \\
\text { Cover }\end{array}$ & $\begin{array}{c}\text { Standard } \\
\text { Error }\end{array}$ & Presence & Prominence \\
\hline \hline LATUPUL6 & 0.25 & 0.25 & 1 & 0.5 \\
\hline HETEVIL6 & 0.38 & 0.13 & 3 & 0.4 \\
\hline CRYPFEN6 & 0.25 & 0.14 & 2 & 0.4 \\
\hline ELYMCAN7 & 0.25 & 0.14 & 2 & 0.4 \\
\hline CORYVIV6 & 0.13 & 0.13 & 1 & 0.3 \\
\hline SALSKAL6 & 0.13 & 0.13 & 1 & 0.3 \\
\hline ARTELUD6 & 0.13 & 0.13 & 1 & 0.3 \\
\hline ARTEFRI6 & 0.13 & 0.13 & 1 & 0.3 \\
\hline OPUNPOL6 & 0.13 & 0.13 & 1 & 0.3 \\
\hline CERAARV6 & 0.13 & 0.13 & 1 & 0.3 \\
\hline KOELMAC7 & 0.13 & 0.13 & 1 & 0.3 \\
\hline \hline MINERAL9 & 55.00 & 2.89 & & \\
\hline ORG9 & 1.75 & 0.25 & & \\
\hline \hline
\end{tabular}

This community was typically found on gentle to moderate southeast to southwest facing dune slopes. The dunes were partially active, however vegetation growth was beginning to stabilize the dune. Typically, there was greater than $50 \%$ exposed sand at the soil surface, with very little organic matter accumulation. A summary of site data is provided in Table 38.

Table 38. Summary of site data for the Sporobolus cryptandrus - Calamovilfa longifoliaOryzopsis hymenoides community type $(n=4)$.

\begin{tabular}{ccccccccc}
\hline \hline Plot & Elevation & Aspect & Slope & Drainage & Site Position & $\begin{array}{c}\text { Surface } \\
\text { Shape }\end{array}$ & $\begin{array}{c}\text { Moisture } \\
\text { Regime }\end{array}$ & Nutrient Regime \\
\hline \hline 1 & 883 & 221 & 16 & Very Rapid & Crest & Straight & Very Xeric & Oligotrophic \\
\hline 3 & 885 & 300 & 27 & Very Rapid & Upper Slope & Straight & Very Xeric & Oligotrophic \\
\hline 4 & 878 & 120 & 9 & Very Rapid & Upper Slope & Straight & Very Xeric & Oligotrophic \\
\hline 15 & 872 & 152 & 5 & Very Rapid & Middle Slope & Straight & Very Xeric & Submesotrophic \\
\hline \hline
\end{tabular}




\subsubsection{Artemisia cana / Stipa comata Association}

Silver sagebrush / needle and thread

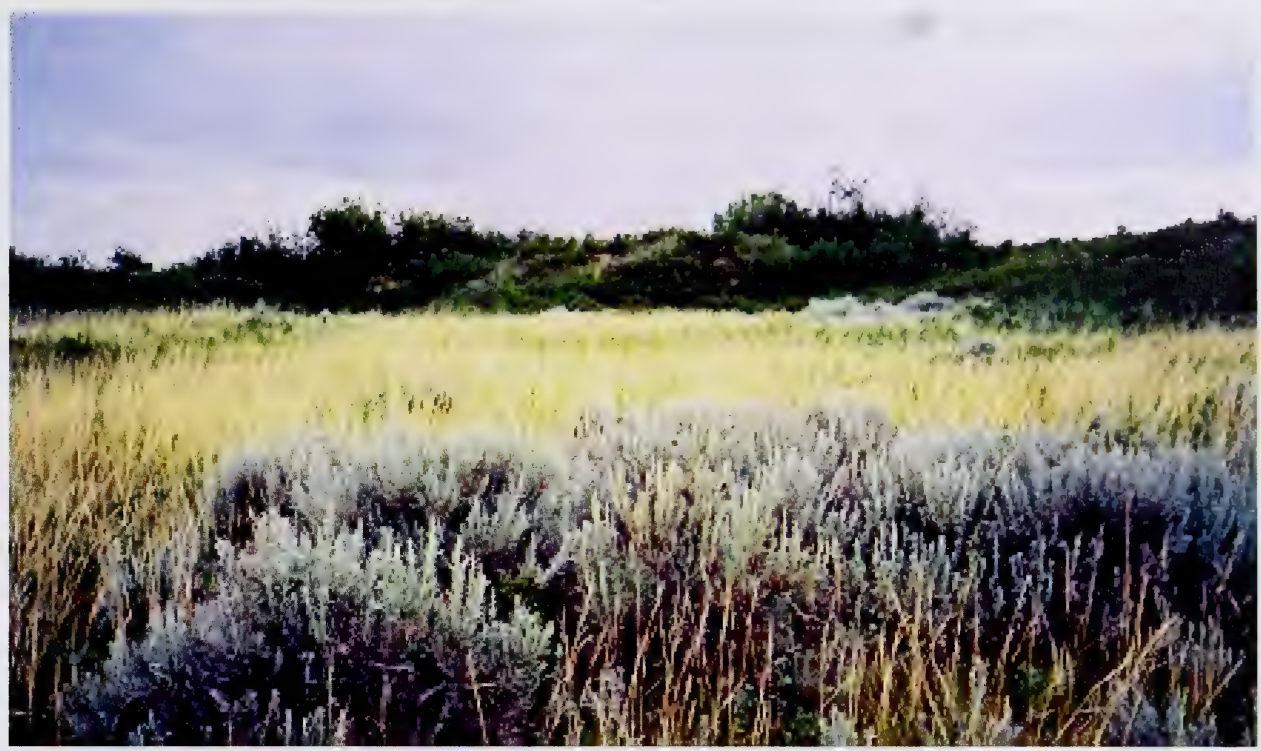

Plate 17. Artemisia cana / Stipa comata community type (Silver sagebrush / needle and thread) Plot 024.

This sand plain community type was dominated by Stipa comata in the grass layer with a sparse low shrub layer, dominated by Artemisia cana, although Koeleria macrantha and Agropyron dasystachyum were also present but with low covers. Other low shrub species were also present at low levels of abundance including Eurotia lanata and Rosa woodsii. Typical forbs included Erigeron caespitosus, Thermopsis rhombifolia, Artemisia frigida and Polycantha vivipara. Table 39 summarizes the species composition and mean percent cover for this community type.

Table 39. Summary statistics for the Artemisia cana / Stipa comata community type (n = 1).

\begin{tabular}{lc}
\hline \hline \multicolumn{1}{c}{ Species } & $\begin{array}{c}\text { Percent } \\
\text { Cover }\end{array}$ \\
\hline \hline STIPCOM7 & 30 \\
\hline ARTECAN5 & 10 \\
\hline ERIGCAE6 & 2 \\
\hline THERRHO6 & 2 \\
\hline ARTEFRI6 & 2 \\
\hline KOELMAC7 & 2 \\
\hline EUROLAN5 & 1 \\
\hline ROSAWOO5 & 1 \\
\hline CORYVIV6 & 1 \\
\hline ANTEPAR6 & 1 \\
\hline \hline
\end{tabular}

\begin{tabular}{lc}
\hline \hline \multicolumn{1}{c}{ Species } & $\begin{array}{c}\text { Percent } \\
\text { Cover }\end{array}$ \\
\hline \hline HETEVIL6 & 1 \\
\hline POTEPEN6 & 0.5 \\
\hline LEPIDEN6 & 0.5 \\
\hline CLEOSER6 & 0.5 \\
\hline ARTELUD6 & 0.5 \\
\hline CHENSUB6 & 0.5 \\
\hline AGRODAS7 & 0.5 \\
\hline \hline MINERAL9 & 10 \\
\hline ORG9 & 0.5 \\
\hline \hline
\end{tabular}


This community was found on a level sand plain and its distribution was quite limited. Soils generally had a higher silt content than any of the other soils encountered in the Pakowki Sandhills study area. There was approximately $10 \%$ exposed mineral soil at the soil surface, with little organic matter accumulation. A summary of site data is provided in Table 40.

Table 40. Summary of site data for the Artemisia cana / Stipa comata community type (n $=1)$.

\begin{tabular}{lcccccccc}
\hline \hline Plot & Elevation & Aspect & Slope & Drainage & Site Position & $\begin{array}{c}\text { Surface } \\
\text { Shape }\end{array}$ & $\begin{array}{c}\text { Moisture } \\
\text { Regime }\end{array}$ & Nutrient Regime \\
\hline \hline 24 & 883 & $\mathrm{n} / \mathrm{a}$ & 0 & Rapid & Level & Straight & Very xeric & Submesotrophic \\
\hline \hline
\end{tabular}




\subsection{Cross-Referencing of Proposed Community Types with Literature}

Each community type identified from the analysis of the plot data was compared against floristically similar community types described for Alberta and other jurisdictions. A summary of the findings are provided in Appendices 4 and 5, which compare the Pakowki Sandhills community types against community types described in literature based in Alberta and other jurisdictions, respectively. A similarity rating between the community types, based on Corns (1983) and recently applied by Strong (2002), is also provided in the tables. A discussion of the community types and associated literature is provided below.

\subsubsection{Populus deltoides / Glycyrrhiza lepidota - Juncus balticus Association}

Western cottonwood / wild licorice - wire rush

Community types, dominated by Populus deltoides have been described for Alberta, though with some variation, particularly in the associated habitat. A floristically similar community type was found occasionally in the Manyberries region, though it was located in moist coulees (Smoliak 1985). A P. deltoides association was also described for CFBSuffield, where it was found on floodplains and alluvial terraces of the South Saskatchewan River (Adams et al. 1997). In a 1993 survey of the Pakowki Sandhills $P$. deltoides was found on sand dunes and plains although a community type was not described in detail. Isolated Populus deltoides were reported to occur in low areas, although aspen clones were reported to be more common (Komex 1993).

Outside Alberta, a floristically similar community type has been described occurring throughout the Great Plains in North America, including Saskatchewan, Montana, Idaho, Wyoming, South and North Dakota and Nebraska. Epp and Townley-Smith (1983) noted Populus deltoides and $P$. tremuloides community types in low areas in sand flats of the Great Sand Hills in Saskatchewan. The community type was reported in locations where soil salinity was low and they also noted that Glycyrrhiza lepidota was an understorey forb species in those locations.

A Populus deltoides - (Salix amygdaloides) / Salix exigua Woodland has been described for numerous locations within the Great Plains region of the United States. At Scotts Bluff National Monument (Nebraska), this community was found on level to gently sloping locations, at the base of low, north-facing slopes. The soils were described as silty rather than sandy and contained more eastern species such as Fraxinus pennsylvanica and Acer negundo (USGS 2002a). At Devil's Tower National Monument (Wyoming) this community was found on river floodplains with sandy soils (USGS $2002 \mathrm{~b}$ ). At this location, the community was very small and had a high cover of invasive, exotic species. Faber-Langendoen (2001) also noted this association in the mid-western United States where it was typically found along the banks of streams and rivers and developed on newly deposited alluvium. This report noted that Glycyrrhiza lepidota was a common forb where site disturbance was low. At Agate Fossil Beds NM (Nebraska) this community was found on the floodplain of the Niobara River. The community type was typically located on level or sloping ground, on the banks or in old channels of the primary floodplain. Soils were described as fine sands and sandy loams that are 
somewhat poorly drained (USGS 2002c). At Badlands National Park, in South Dakota this community was found along river and creek floodplains, pond and reservoir margins, seeps and springs in mesic draws and seeps and springs that occur along the edge of sandhill complexes (USGS 2002d).

According to Natureserve (2002) " This cottonwood - willow woodland is found widely in the central Great Plains of the United States. Stands occur on recently deposited alluvial material along rivers and streams. The soils are derived from alluvial sand, silt and clay and are poorly developed. The water table fluctuates with the level of the adjacent river or stream. Populus deltoides is the dominant species in this community, although Salix exigua and/or Salix interior is generally more dominant in the initial stage following a major flood event. Salix amygdaloides is rare to codominant. The shrub/sapling layer is conspicuous, especially near the stream bank and consists mainly of Salix exigua, Populus deltoides and Salix amygdaloides, or occasionally Salix lutea. In the more easterly parts of the range, Salix interior may replace Salix exigua. On the older margins of this community Fraxinus pennsylvanica is often found as a sapling or small canopy tree. The herbaceous stratum is variable. Graminoids typical of undisturbed sites include Carex emoryi, Carex pellita (= Carex lanuginosa), Pascopyrum smithii and Spartina pectinata. Equisetum arvense and Glycyrrhiza lepidota are common forbs in these sites. Widely distributed species that are adapted to these sites include Ambrosia psilostachya, Artemisia campestris ssp. caudata, Artemisia ludoviciana, Calamovilfa longifolia, Cenchrus longispinus, Chamaesyce serpyllifolia (= Euphorbia serpyllifolia), Euphorbia esula, Grindelia squarrosa, Helianthus petiolaris, Heterotheca villosa, Phyla lanceolata (= Lippia lanceolata), Opuntia macrorhiza, Poa pratensis and Sporobolus cryptandrus. These sites are prone to invasion by exotic grasses and forbs, the most widely established being Agrostis stolonifera, Bromus tectorum, Cirsium arvense, Bassia scoparia (= Kochia scoparia), Melilotus spp., Taraxacum officinale and Tragopogon dubius." It was also noted that many sites were overgrazed and invaded by exotic woody and herbaceous species.

A Populus deltoides / Pascopyrum smithii woodland was found in Thunder Basin National Grassland (Wyoming) along the Cheyenne River (Jones 1998a). In this location, the community type forms linear groves of trees that run parallel to the stream course on alluvial materials. A Populus deltoides / Calamovilfa longifolia woodland was also described in Thunder Basin National Grassland, along Antelope Creek (Jones 1998b).

A Populus deltoides / Symphoricarpos occidentalis Woodland was found in the Bitter Creek Badlands region of Montana, where it was found along rivers on unstabilized floodplains where it colonized alluvial deposits on meanders of streams and rivers (Cooper et al. 2001). In this location, the authors noted that a well-developed shrub layer was typically present. A Populus tremuloides / Symphoricarpos alba forest has been described from the Medicine Lake Sandhills in Montana (Heidel et al. 2000). There was no $P$. deltoides observed at this site and the community type did not have a native understorey. 


\subsubsection{Rosa woodsii / Sporobolus cryptandrus Association}

Common wild rose / sand dropseed

Rosa woodsii was also found on dunes in the 1993 during a survey of the Pakowki Sandhills (Komex 1993). The species was reported to occur in low areas between dunes, often in association with wild licorice, although a full community description was not provided.

Outside Alberta, a shrubland, dominated by Elaeagnus commutata, Rosa woodsii, Symphoricarpos occidentalis or Prunus virginiana was noted for the Great Sandhills in Saskatchewan (Epp and Townley-Smith 1980). They noted that these species formed closed shrublands on stabilized slip faces that were typically north facing. The shrub composition varied from location to location and each shrub species could be dominant at specific sites, where at others the species were co-dominant. Furthermore, Epp and Townley-Smith (1980) noted a Rosa woodsii community that was dominant in areas "where sand has been whipped off dune by strong winds, creating areas of slow sand accumulation." Coupland (1950) noted that a Rosa woodsii (Artemisia cana / Elaeagnus commutata) community type was found in undulating to gently rolling areas between stabilized dunes in the prairie provinces. He noted that the water table was typically within eight to twelve feet of the soil surface where this community was found. Thorpe and Godwin (1993) noted a grass and shrub / sand type in the Manito Sand Hills of Western Saskatchewan. While this community type was dominated by Symphoricarpos occidentalis, Rosa spp. and Prunus virginiana were also present in 50\% of plots in this type. The community type was typically found on stabilized dunes, with rapidly drained, sandy soils. However, Sporobolus cryptandrus was not listed as a prevalent species.

Natureserve (2002) notes a Rosa woodsii Temporarily Flooded Shrubland Alliance. These shrublands were typically found in the foothills and plains of Montana and Idaho. Elevations for the community type ranged from $650-1700 \mathrm{~m}$ and typically occurred on floodplains and alluvial terraces along rivers and streams. This alliance was also located on hillsides below springs and in ravines and swales where overland flow from snowmelt and summer precipitation provided additional moisture. Sites were generally flat to moderately steep and had soils that ranged from sandy loams to silt loams. Although these sites are considered temporarily flooded, they were well drained and were not found to have a shallow water table.

Cooper et al. (1999) noted a Rosa woodsii Shrubland community type in Beaverhead Mountain region of Montana. Although it was listed as a community type for this area, it was not described in detail in the associated report. The Montana and Idaho natural heritage programs (MNHP 2002; Rust 1997) listed a Rosa woodsii Shrubland as a natural plant community for their states. However, detailed descriptions of the community and associated habitats were not provided. 


\subsubsection{Salix amygdaloides - Rosa woodsii / Juncus balticus - Sporobolus cryptandrus Association}

Peach-leaf willow - common wild rose / wire rush - sand dropseed

Salix amygdaloides was reported on dunes during a 1993 survey of the Pakowki Sandhills (Komex 1993). It was typically found in low areas, in both a tree and shrub form. Unfortunately, a community description was not provided. A general Salix spp. / Stipa comata association was reported for CFB-Suffield, where it was found on floodplains and alluvial terraces of the South Saskatchewan River (Jaques 1977). No reports of a similar community could be found for other Canadian provinces.

A Salix amygdaloides Woodland type was reported by Natureserve (2002) and was found in the Northern Rocky Mountains and potentially into parts of the western Great Plains. Stands occurred in riparian areas and the vegetation was dominated by Salix amygdaloides. Specifically, the Salix amygdaloides Woodland was documented in the Black Hills of South Dakota at the confluence of two creeks where it formed a tall-shrub stratum with Salix bebbiana and Cornus sericea and was more a shrubland than a woodland (Faber-Langendoen 2001; Marriott and Faber-Langendoen 2000).

A Salix amygdaloides Woodland was listed as a natural plant community for Montana where it was ranked G3/S3 by the Montana Natural Heritage Program (2002).

A Salix amygdaloides / Salix exigua Woodland was also reported by Natureserve (2002) where the association occurred in riparian habitats on the Columbian Plateau in the interior Northwest and in northeastern Utah. The association was found at elevations ranging from 100-1600 $\mathrm{m}$ and was located in overflow channels of large rivers and on narrow floodplains of small creeks. Soil textures were reported to be quite variable, but did not include clay. This community has been reported to have a moderately open canopy dominated by the small tree Salix amygdaloides. Salix exigua dominated the tallshrub layer. Other tree species reported for this community type included Populus fremontii, Acer negundo, Populus angustifolia, Populus deltoides and the introduced Elaeagnus angustifolia.

\subsubsection{Elaeagnus commutata / Glycyrrhiza lepidota Association \\ Silverberry / wild licorice}

An Elaeagnus commutata Shrubland was identified during a 1993 survey of the Pakowki Sandhills (Komex 1993). This community was reported to occur as thickets on some north-facing slopes and at the base of some dunes. However, a detailed description of the community type was not provided. An Elaeagnus commutata community has been described for Alberta based on three reports in Central Parkland, at Dillberry Lake Provincial Park, near Rumsey and in Dry Island Buffalo Jump (Wheatley and Bentz 2002). This community occurred as low shrublands and shrubby meadows along the perimeter of saline lakes, adjacent to marshes and graminoid meadows. At Dillberry Lake Provincial Park, Elaeagnus commutata occurred as a shrubland primarily with Symphoricarpos occidentalis, Rosa acicularis, Calamovilfa longifolia, Agropyron trachycaulum and Carex spp.. As a meadow community, E. commutata was more common with Rosa woodsii, Glycyrrhiza lepidota, Juncus balticus and Calamovilfa 
longifolia. An Elaeagnus commutata / Symphoricarpos occidentalis - Rosa woodsii / Poa palustris community was also described from Central Parkland occurring near Wainwright on subxeric to submesic dune sites with good internal soil drainage. Currently, ANHIC lists an Elaeagnus commutata / Pascopyrum smithii Shrubland community for the Grassland Natural Region and it is ranked S3 (Allen 2002).

Epp and Townley-Smith (1980) reported an Elaeagnus commutata - Rosa woodsii Symphoricarpos occidentalis - Prunus virginiana closed shrubland in the Great Sand Hills of Saskatchewan. The community was typically found on stabilized slip faces that were north facing. They noted that the shrub composition varied from location to location and that each shrub species could be dominant at specific sites, where at others they may be co-dominant. The authors also noted an Elaeagnus commutata shrubland that was often found inhabiting blowouts away from bare sand (Epp and Townley-Smith 1980). Coupland (1950) described a Rosa woodsii (Artemesia cana / Elaeagnus commutata) community type for the prairie provinces that was found in undulating to gently rolling areas between stabilized dunes. At these locations, the water table was typically within 8 to 12 feet of the soil surface.

Heidel et al. (2000) reported an Elaeagnus commutata Shrubland community for northern Montana, located east of the Continental divide. The community type was generally classified as temporarily flooded. At the Medicine Lake sandhills, sites typically had a shrub cover of $10 \%$ and grass cover of $70 \%$ and sites were not flooded but the water table was within the rooting zone.

An Elaeagnus commutata Shrubland Alliance was reported by Natureserve (2002) for the northern Great Plains in a mixedgrass prairie matrix. The alliance was dominated by mid to tall shrubs, particularly Elaeagnus commutata. Pascopyrum smithii was reported as the dominant species in the herbaceous layer and was typically accompanied by Koeleria macrantha, Schizachyrium scoparium and Hesperostipa comata. They also note that Elaeagnus commutata was most abundant on flat sandy sites in southern Saskatchewan.

Natureserve (2002) also reported an Elaeagnus commutata / Pascopyrum smithii Shrubland Association, within the shrubland alliance that typically occurred on a variety of glacial landforms including kames, eskers and areas of till and outwash. The association was most common on north-facing slopes and sites where moisture is more abundant, including river valley slopes.

An Elaeagnus commutata Shrubland was listed as a natural plant community for Montana and was ranked G2Q/S2? by the Montana Natural Heritage Program (2002). Unfortunately no description of the community type is given.

\subsubsection{Elaeagnus commutata / Artemisia ludoviciana - Calamovilfa longifolia Association}

Silverberry / prairie sagewort - sand grass

An Elaeagnus commutata Shrubland was identified during a 1993 survey of the Pakowki Sandhills (Komex 1993). This community was reported to occur as thickets on some north-facing slopes and at the base of some dunes. However, a detailed description of the community type was not provided. An Elaeagnus commutata community has been 
described for Alberta based on three reports in Central Parkland, at Dillberry Lake Provincial Park, near Rumsey and in Dry Island Buffalo Jump (Wheatley and Bentz 2002). This community occurred as low shrublands and shrubby meadows along the perimeter of saline lakes, adjacent to marshes and graminoid meadows. At Dillberry Lake Provincial Park, Elaeagnus commutata occurred as a shrubland primarily with Symphoricarpos occidentalis, Rosa acicularis, Calamovilfa longifolia, Agropyron trachycaulum and Carex spp.. As a meadow community, E. commutata was more common with Rosa woodsii, Glycyrrhiza lepidota, Juncus balticus and Calamovilfa longifolia. An Elaeagnus commutata / Symphoricarpos occidentalis - Rosa woodsii / Poa palustris community was also described from Central Parkland occurring near Wainwright on subxeric to submesic dune sites with good internal soil drainage. Currently, ANHIC lists an Elaeagnus commutata / Pascopyrum smithii Shrubland community for the Grassland Natural Region and it is ranked S3 (Allen 2002).

Epp and Townley-Smith (1980) reported an Elaeagnus commutata - Rosa woodsii Symphoricarpos occidentalis - Prunus virginiana closed shrubland in the Great Sand Hills of Saskatchewan. The community was typically found on stabilized slip faces that were north facing. They noted that the shrub composition varied from location to location and that each shrub species could be dominant at specific sites, where at others they may be co-dominant. The authors also noted an Elaeagnus commutata shrubland that was often found inhabiting blowouts away from bare sand (Epp and Townley-Smith 1980). Coupland (1950) described a Rosa woodsii (Artemesia cana / Elaeagnus commutata) community type for the prairie provinces that was found in undulating to gently rolling areas between stabilized dunes. At these locations, the water table was typically within 8 to 12 feet of the soil surface.

Heidel et al. (2000) reported an Elaeagnus commutata Shrubland community for northern Montana, located east of the Continental divide. The community type was generally classified as temporarily flooded. At the Medicine Lake sandhills, sites typically had a shrub cover of $10 \%$ and grass cover of $70 \%$ and sites were not flooded but the water table was within the rooting zone.

An Elaeagnus commutata Shrubland Alliance was reported by Natureserve (2002) for the northern Great Plains in a mixedgrass prairie matrix. The alliance was dominated by mid to tall shrubs, particularly Elaeagnus commutata. Pascopyrum smithii was reported as the dominant species in the herbaceous layer and was typically accompanied by Koeleria macrantha, Schizachyrium scoparium and Hesperostipa comata. They also note that Elaeagnus commutata was most abundant on flat sandy sites in southern Saskatchewan.

Natureserve (2002) also reports an Elaeagnus commutata / Pascopyrum smithii Shrubland Association, within the shrubland alliance that typically occurred on a variety of glacial landforms including kames, eskers and areas of till and outwash. The association was most common on north-facing slopes and sites where moisture is more abundant, including river valley slopes.

An Elaeagnus commutata Shrubland was listed as a natural plant community for Montana and was ranked G2Q/S2? by the Montana Natural Heritage Program (2002). Unfortunately no description of the community type is given. 


\subsubsection{Prunus virginiana / Calamovilfa longifolia Association}

Choke cherry / sand grass

A 1993 survey of the Pakowki Sandhills reported a Prunus virginiana shrubland (Komex 1993). The authors of this survey noted that this community typically occurred along low dune ridges and slopes. A Prunus virginiana - Amelanchier alnifolia / Agropyron trachycaulum - Poa pratensis community was described for the Central Parkland (Wheatley and Bentz 2002). This community was found on a steep, south-facing slope in the Blackfoot Provincial Recreation Area.

Epp and Townley-Smith (1980) reported an Elaeagnus commutata - Rosa woodsii Symphoricarpos occidentalis - Prunus virginiana closed shrubland in the Great Sand Hills of Saskatchewan. The community was typically found on stabilized slip faces that were north facing. They noted that the shrub composition varied from location to location and that each shrub species could be dominant at specific sites, where at others they may be co-dominant. Thorpe and Godwin (1993) reported a grass and shrub / sand type that was dominated by Symphoricarpos occidentalis, however, Rosa spp. and Prunus virginiana were present in $50 \%$ of plots. This community type was typically found on stabilized dunes, with rapidly drained, sandy soils and Calamovilfa longifolia was listed as a common graminoid species.

Heidel et al. (2000) and the Montana Natural Heritage Program (2002) both report a Prunus virginiana Shrubland in Montana. The Natural Heritage Program does not provide a description of the community but gives it a rank of $\mathrm{G} 4 \mathrm{Q} / \mathrm{S} 4$. Heidel et al. (2000) noted that the shrubland was small in area when it occurred in the Medicine Lake Sandhills. They noted that the community type typically had a high shrub cover and low understorey cover due to the density of shrubs, although Hesperostipa comata was a common understorey graminoid species. Prunus virginiana has a deep root system, allowing the shrubs to reach the water table in the Medicine Lake Sandhills. Elsewhere in Montana the community type was considered to be a riparian community (Heidel et al. 2000).

Cooper et al (1999) reported a Prunus virginiana - (Prunus americana) Shrubland community in Montana. It was found in the Bitter Creek Badlands, located at heads of coulees feeding into badlands. The community occurred as very small patches, often linear in shape and very dense. Very few other species were present because of the density of the Prunus.

Natureserve (2002) reported a Prunus virginiana Shrubland Alliance. The community was typically found along streams, rivers, lakes and ponds and on terraces, or in canyons or steep gullies where elevations range from $716 \mathrm{~m}$ to about $1600 \mathrm{~m}$ in Montana, Wyoming and Colorado and up to $2440 \mathrm{~m}$ in Nevada. In certain locations, the alliance occurred on side slopes, immediately below a seep or spring. Some examples of this alliance have been classified as having an intermittently or temporarily flooded hydrologic regime. Soils were typically well developed and well drained and were typically composed of shallow to deep alluvial deposits.

A Prunus virginiana - (Prunus americana) Shrubland was reported occurring in Badlands National Park, South Dakota (USGS 2002d). This community, however, was dominated by Prunus americana, with some $P$. virginiana. It was typically found in 
sloping to nearly level mesic draws and nearly level oxbows, although a few stands were also found at seep zones on the edge of sandhills (USGS 2002d).

\subsubsection{Eurotia lanata / Stipa comata - Calamovilfa longifolia Association}

Winter fat / needle and thread - sand grass

Within Alberta, Smoliak (1985) reported a Stipa - Bouteloua - Agropyron type near Manyberries, in southern Alberta. The author noted that this type was typically found on upland prairie, with Eurotia lanata as a common shrub.

Looman (1980) recorded a Stipa comata Association for the prairie provinces in Canada. The author noted that this community was found to be common (with Bouteloua gracilis) on dry prairie. A variation of the community was also noted and included Calamovilfa longifolia, occurring on sandy loam or loamy sand soils. Eurotia lanata was not mentioned as occurring.

Natureserve (2002) reported a Krascheninnikovia lanata / Hesperostipa comata DwarfShrubland Alliance. It was considered to be a minor alliance and included dwarfshrublands scattered across the interior western U.S. Stands occur on plateaus, plains, mesas, hillslopes, alkaline flats around playas and along drainages. Some habitats were intermittently flooded wetlands. It was typically found on flat to gently sloping sites occurring on any aspect, but stands have also been reported from moderately steep slopes. Soils were commonly calcareous to moderately alkaline and are typically stony, sandy loam. The ground cover was mostly bare soil. The cover of Krascheninnikovia lanata and Hesperostipa comata varied from 5 to $60 \%$. The vegetation was reported to be sparse in many of these stands and might be better classified in a sparsely vegetated alliance.

The Montana Natural Heritage Program (2002) reported a Krascheninnikovia lanata / Stipa comata Dwarf-Shrubland community for Montana and ranked it as G3/S3. A Eurotia lanata / Poa secunda extremely xeromorphic dwarf-shrubland was listed as a natural plant community type for Idaho (Rust 1997) 3. Unfortunately no descriptions were given for either of these communities.

Faber-Langendoen (2001) reported a Krascheninnikovia lanata / Bouteloua gracilis Dwarf Shrub Herbaceous community type for the mid-western United States. The vegetation contained open shrub and graminoid layers, where the short herbaceous layer was dominated by Bouteloua gracilis, Echinacea angustifolia and Liatris punctata. This community type was found in the southwestern Great Plains and semi-desert mountains, from Colorado south to New Mexico and Arizona and was also reported in Kansas.

\subsubsection{Salix exigua / Glycyrrhiza lepidota - Juncus balticus Association}

Sandbar willow / wild licorice - wire rush

No community types with a similar plant species composition could be found for Alberta. However, many have been described for the Great Plains region in the United States.

Faber-Langendoen (2001) described a Salix exigua / Mesic Graminoids Shrubland for the Midwestern United States. For this community type, the vegetation was dominated by 
shrubs with a fairly dense (at least $30 \%$ ) ground cover of mesic graminoids and forbs. Juncus spp. was noted as a common herbaceous species. The community was most commonly found on sandbars, islands and shorelines of stream channels and braided rivers and soils are typically poorly developed. This community was predominantly found in the Great Plains but also in parts of Rocky Mountains and intermountain semidesert regions, from Wyoming west to possibly Idaho, south to Utah and east to Oklahoma. The author also reported a Salix exigua Temporarily Flooded Shrubland community that was dominated by $2-4 \mathrm{~m}$ tall Salix exigua with a moderate to high stem density. This community was dominantly found on recently deposited or disturbed alluvial materials, composed primarily of sands. It was found at lower elevations throughout the northwestern US and Great Plains and into Manitoba.

Cooper et al. (1999) noted a Salix exigua Temporarily Flooded Shrubland community in the Beaverhead Mountains of Montana. At this location, the community was typically found on gravelly alluvial materials on floodplains and terraces in river bottoms. Understorey species were minimal due to the high disturbance rate, but the most common were reported to be Cirsium arvense, Mentha arvensis and Phalaris arundinacaea.

Several natural plant community types, dominated by Salix exigua, were reported by the Montana Natural Heritage Program (2002). They included a Salix exigua / Barren Shrubland, a Salix exigua / Mesic Graminoid Shrubland and a Salix exigua Temporarily Flooded Shrubland. Unfortunately no descriptions of the community types were provided but all were ranked as G5/S5.

Several natural plant community types dominated by Salix exigua were reported for the State of Idaho (Rust 1997) and are listed below:

> Salix exigua / Barren seasonally flooded cold-deciduous shrubland,

> Salix exigua / Equisetum arvense seasonally flooded cold-deciduous shrubland,

$>$ Salix exigua / Mesic Forb seasonally flooded cold-deciduous shrubland,

> Salix exigua / Mesic Graminoid seasonally flooded cold-deciduous shrubland and

> Salix exigua / Rosa woodsii seasonally flooded cold-deciduous shrubland.

Unfortunately, no detailed descriptions were provided for these community types.

A Salix exigua Shrubland community, has been described from the margins of the North Platte River at Scotts Bluff National Monument in Nebraska (USGS 2002a) This community type was reported to occur on recently deposited alluvial sands where there was little soil development. The understorey species composition was quite different from that found at the Pakowki Sandhills and the occasional Populus deltoides was also noted to occur. A similar Salix exigua Shrubland [Provisional] community was found along the Niobara River, at the Agate Fossil Beds NM in Nebraska (USGS 2002c). At this location, the community was found along lower floodplain terraces, with sandy loam soils that were poorly to somewhat poorly drained. Juncus balticus was listed as an abundant species and the authors noted that species diversity was quite high.

A Salix exigua Temporarily Flooded Shrubland community has been described from Badlands National Park, in South Dakota (USGS 2002d) At this location, the community was found along banks of several creeks within the park. The authors noted that the sites for this plant community were nearly all level and that ground water was present. 


\subsubsection{Glycyrrhiza lepidota - Calamovilfa longifolia Association}

Wild licorice - sand grass

A 1993 survey of the Pakowki Sandhills reported the occurrence of Glycyrrhiza lepidota with Calamovilfa longifolia and Artemesia ludoviciana (Komex 1993). Although a detailed description of the community type was not provided, the authors noted that wire rush (Juncus balticus) was also commonly found in Glycyrrhiza lepidota sites.

The only reported Glycyrrhiza lepidota community type from outside Alberta was found in Montana. The Montana Natural Heritage Program (2002) recorded a Glycyrrhiza lepidota Herbaceous Vegetation community for the state and it is currently ranked as $\mathrm{S}$ ? Unfortunately, no detailed description of the plant community was provided.

\subsubsection{Glycyrrhiza lepidota - Artemisia spp. - Stipa comata Association}

Wild licorice - sage - needle and thread

A 1993 survey of the Pakowki Sandhills reported the occurrence of Glycyrrhiza lepidota with Calamovilfa longifolia and Artemesia ludoviciana (Komex 1993).

Looman (1980) recorded a Stipa comata Association for the prairie provinces in Canada. The author noted that this community was found to be common (with Bouteloua gracilis) on dry prairie. A variation of the community was also noted and includes Calamovilfa longifolia, occurring on sandy loam or loamy sand soils, although Glycyrrhiza lepidota was not noted to occur.

Hulett et al. (1966) recorded a Stipa comata - Artemesia frigida plant community that was dominant on stabilized dunes in Great Sand Hills of Saskatchewan Glycyrrhiza lepidota was not reported to be present in the community.

The only reported Glycyrrhiza lepidota community type from outside Alberta was found in Montana. The Montana Natural Heritage Program (2002) recorded a Glycyrrhiza lepidota Herbaceous Vegetation community for the state and it is currently ranked as S? . Unfortunately, no detailed description of the plant community was provided.

\subsubsection{Rumex venosus Association}

Wild begonia

No community types with a similar plant species composition could be found for Alberta. However, several have been described for areas outside Alberta, but not in the United States.

Looman (1980) described a Rumex venosus Alliance that was dominant on highly mobile dunes, during early stages of development. Epp and Townley-Smith (1980) also noted that Rumex venosus occurred on active sand dune complexes, typically towards the edge of the deflation zone and on sides of dunes in their Active Sand Dune Complex. However, no Rumex venosus community type was described. 


\subsubsection{Oryzopsis hymenoides - Sporobolus cryptandrus Association}

Indian rice grass - sand dropseed

Only one community type with a somewhat similar plant species composition could be found for Alberta. Fehr (1984) reported a Carex foenea - Calamovilfa longifolia Elymus canadensis - Oryzopsis hymenoides community in the Wainwright sand dune area, on active blowouts. ANHIC reported an Oryzopsis hymenoides / Leymus canadensis sparsely vegetated plant community that they have ranked as S2 for the province (Allen 2002), but no detailed description was provided.

Outside Alberta, there are numerous reports of related community types. Looman (1980) reported an Oryzopsis hymenoides Order within the Calamovilfetea class for the Canadian prairie provinces, though no details were provided on its species composition or the associated habitat. Hulett et al. (1966) reported a Psoralea lanceolata-Oryzopsis hymenoides community that was a dominant association in the Great Sand Hills (Saskatchewan) on active sand dune complexes. Epp and Townley-Smith (1980) also reported a Psoralea lanceolata - Oryzopsis hymenoides community type from the Great Sand Hills where it formed a sparse cover located on the edge of deflation zones away from the active portions of the dune. The authors noted that the vegetative cover tended to increase as the distance from deflation zone increased.

In Montana, Heidel et al. (2000) reported an Oryzopsis hymenoides - Psoralidium lanceolatum community in the Medicine Lake Sandhills in Montana, that was restricted to slopes and crests of sand dunes recently disturbed by soil erosion. Sporobolus cryptandrus was also present and often formed up to $10 \%$ cover. The community type was most commonly found in blowouts and was thought to be the driest and earliest stage of succession.

An Oryzopsis hymenoides - Psoralidium lanceolatum Herbaceous vegetation association was reported to be a natural plant community for the state of Montana, where it was ranked G3Q/S? (MNHP 2002).

An Achnatherum hymenoides Herbaceous Alliance was reported by Natureserve (2002) where it occurred in two distinctively different habitats: sandy areas and shale barrens, in different geographic areas. Sandy areas included 'blowouts' in the Great Plains and in arid and semi-arid dune systems in the Chihuahua Desert, San Luis Valley, Colorado Plateau and Great Basin. Substrates were dominantly sand or shale. This alliance was characterized by a sparse to moderately dense herbaceous layer that was dominated by Achnatherum hymenoides (= Oryzopsis hymenoides). An Achnatherum hymenoides Psoralidium lanceolatum Herbaceous Vegetation association and a Calamovilfa longifolia - Achnatherum hymenoides Herbaceous Vegetation association was also listed by Natureserve (2002) but no descriptions were given.

\subsubsection{Stipa comata - Oryzopsis hymenoides Association}

Needle and thread - Indian rice grass

Only one community type with a somewhat similar plant species composition could be found for Alberta. Fehr (1984) reported a Carex foenea - Calamovilfa longifolia - 
Elymus canadensis - Oryzopsis hymenoides community in the Wainwright sand dune area, on active blowouts. However, Stipa comata was not listed as a component of this community.

Looman (1980) recorded a Stipa comata Association for the prairie provinces in Canada. The author noted that this community was found to be common (with Bouteloua gracilis) on dry prairie. A variation of the community was also noted and includes Calamovilfa longifolia, occurring on sandy loam or loamy sand soils although Achnatherum hymenoides was not noted to occur. Looman (1980) also reported an Oryzopsis hymenoides Order within the Calamovilfetea class for the Canadian prairie provinces, though no details are provided on its species composition or the associated habitat. Hulett et al.(1966) reported a Psoralea lanceolata - Stipa comata community that was a dominant association in Dundurn Sand Hills (Saskatchewan) on stabilized dune complexes. Hulett et al.(1966) reported a Psoralea lanceolata - Oryzopsis hymenoides community that was a dominant association in the Great Sand Hills (Saskatchewan) on active sand dune complexes. This same report also recorded a Stipa comata - Artemesia frigida plant community that was dominant on stabilized dunes in the Great Sand Hills of Saskatchewan.

Epp and Townley-Smith (1980) also reported a Psoralea lanceolata - Oryzopsis hymenoides community type from the Great Sand Hills where if formed a sparse cover located on the edges of deflation zones away from the active portions of the dune. The authors noted that the vegetative cover tended to increase as the distance from the deflation zone increased.

Heidel et al. (2000) reported a Stipa comata / Psoralidium lanceolatum Herbaceous Vegetation association from the Medicine Lake sand hills in Montana. This community was restricted to wind-blown sand deposits with undeveloped soils and was found on choppy dunes to rolling plains. It was thought to be a seral stage between the Oryzopsis hymenoides / Psoralidium lanceolatum and the Pascopyrum smithii - Stipa comata association. Grass cover was typically $20-40 \%$ and most was composed of Stipa comata. This community was most common on stabilized to partially stabilized sand dunes. An Oryzopsis hymenoides - Psoralidium lanceolatum Herbaceous vegetation association was reported to be a natural plant community for the state of Montana, where it is ranked G3Q/S? (MNHP 2002).

Natureserve (2002) reported an Hesperostipa comata - Achnatherum hymenoides Herbaceous Vegetation association. This grass type has been reported from the Great Divide Basin in south-central Wyoming. Hesperostipa comata and Achnatherum hymenoides co-dominated the graminoid stratum, while Pascopyrum smithii was a secondary species. Scattered shrubs were present, primarily Artemisia tridentata ssp. wyomingensis. This type apparently has not been described outside the Great Divide Basin of south-central Wyoming. Other basins in south-central and southwestern Wyoming and the northwestern quarter of Colorado are similar in climate and geology and this association may well extend over a wide area of the two states.

An Hesperostipa comata Bunch Herbaceous Alliance was also reported by Natureserve (2002). This grassland alliance was found on sandy soils in the Intermountain Steppe, Wyoming Basin, Colorado Plateau, Great Basin and Columbia Plateau. The community 
typically occurred on upland sites with coarse-textured soils such as sandstone outcrop ridges in the plains, dry-sandy sites in the Columbia Basin and on dissected alluvial fans below sandstone plateaus, but not on dunes.

\subsubsection{Artemisia spp. - Stipa comata - Calamovilfa longifolia Association}

Sage - needle and thread - sand grass

Smoliak (1985) reported a Calamovilfa longifolia - Artemisia community on upland prairie with sandy soils in the Manyberries region of southern Alberta. However, no other description of the community type was provided.

Looman (1980) recorded a Stipa comata Association for the prairie provinces in Canada. The author noted that this community was found to be common (with Bouteloua gracilis) on dry prairie. A variation of the community was also noted and included Calamovilfa longifolia, occurring on sandy loam or loamy sand soils. However, no mention of Artemisia spp. being a component of this community was noted.

Hulett et al. (1966) recorded a Stipa comata - Artemesia frigida plant community that was dominant on stabilized dunes in the Great Sand Hills of Saskatchewan, but did not elaborate on its composition or distribution.

\subsubsection{Stipa comata - Cyperus schweinitzii - Calamovilfa longifolia Association}

Needle and thread - sand nut-grass - sand grass

Adams et al. (1997) described a similar Calamovilfa longifolia - Hesperostipa comata community from CFB Suffield, in southeastern Alberta. Site conditions differed in that this community was described as occurring on fluvial slump features along the South Saskatchewan River, where a sand veneer was present. Soils were typically loamy sand and slopes ranged from 5 to $15 \%$, although some $60 \%$ slopes were reported. This community type was also found on glaciofluvial outwash plains, with minimal eolian action. Soil textures were loamy sand and slopes ranged from 0 to 5\%. Smoliak (1985) reported two similar community types in the Manyberries area of southern Alberta. The author described a Calamovilfa longifolia - Artemesia plant community that was found on upland prairie with sandy soils and a Calamovilfa longifolia - Stipa comata community found on sandy soils, in coulee bottoms.

Looman (1980) recorded a Stipa comata Association for the prairie provinces in Canada. The author noted that this community was found to be common (with Bouteloua gracilis) on dry prairie. A variation of the community was also noted and includes Calamovilfa longifolia, occurring on sandy loam or loamy sand soils.

Hulett et al. (1966) recorded a Stipa comata - Artemesia frigida plant community that was dominant on stabilized dunes in the Great Sand Hills of Saskatchewan and noted that Calamovilfa longifolia was present. Hulett et al. (1966) also described a Psoralea lanceolata - Stipa comata plant community that was dominant at the Dundurn Sand Hills (Saskatchewan) on stabilized dunes. Calamovilfa longifolia was reported to be present in this community. Furthermore, the authors described a Stipa comata - Calamovilfa longifolia - Agropyron spp. community that was found in the Dundurn Sand Hills. The 
authors noted that this community was most common in dune depressions and appeared to be intermediate between stabilized blowouts and stabilized dunes.

Cooper et al. (2001) reported a Calamovilfa longifolia - Hesperostipa comata Herbaceous Vegetation association from the Rock Creek Canyon and a few sandy outcrops in the Bitter Creek Badlands area of Montana. This community was found to occur most abundantly where sandy substrates were dominant, on stabilized dunes, interdunal swales and colluvial sands. The authors reported that the community was found to be highly restricted in area and occurred mainly on colluvial sands.

Heidel et al. (2000) also reported a Calamovilfa longifolia - Stipa comata Herbaceous Vegetation association in the Medicine Lake Sandhills in Montana. The authors considered it to be a minor type. It did not appear to be a widespread community in the Medicine Lake Sandhills, occurring only in small patches on low ridges and in a mosaic pattern on gentle plains of Medicine Lake.

Jones (1998a; 1998b) noted Calamovilfa longifolia - Hesperostipa comata Grassland communities along several rivers and streams in northeastern Wyoming. The author described this community type on sandy soils at an intermediate height above the river channel and noted it was a major community type in the region. Other dominant species included Calamovilfa longifolia, Stipa comata and Psoralea lanceolata.

Faber-Langendoen (2001) and Natureserve (2002) reported a Calamovilfa longifolia Hesperostipa comata Herbaceous Vegetation community from the mid-western United States. The vegetation had an open canopy dominated by Calamovilfa longifolia and Hesperostipa comata. This community was found to occur on stabilized sand dunes as well as in interdunal valleys or draws, colluvial sands and less commonly on silty terraces of intermittent streams. Soils were generally medium to fine sands, formed from either eolian or colluvial processes. The author noted that this community was found in the central and northern Great Plains, ranging from Colorado to Nebraska and north to Wyoming and South Dakota.

\subsubsection{Sporobolus cryptandrus - Calamovilfa longifolia - Oryzopsis hymenoides Association}

Sand dropseed - sand grass - Indian rice grass

Wheatley and Bentz (2002) and Meijer and Karpuk (1999) described a Sporobolus cryptandrus - Calamovilfa longifolia - Koeleria macrantha - Carex obtusata community type from the Central Parkland of Alberta. This community type was found on active dunes and blowouts at Dillberry Lake Provincial Park where it was generally located on south to west-facing aspects and had a sparse vegetative cover. Heterotheca villosa was also reported to be present. In a 1993 survey of the Pakowki Sandhills, a Calamovilfa longifolia - Sporobolus cryptandrus sparsely vegetated active sand dune complex was reported (Komex 1993). Other reported species included Helianthus couplandii, Thermopsis rhombifolia and Heterotheca villosa.

ANHIC currently ranks Sporobolus cryptandrus sparsely vegetated active dune plant communities as S2 (Allen 2002). 
Looman (1980) described a Calamovilfa longifolia vegetation Class from the Canadian prairie provinces. This class was reported to be dominant in well-developed sandhill prairie and could include other characteristic species such as Elymus canadensis, Helianthus couplandii and Sporobolus cryptandrus.

Thorpe and Godwin (1993) described a Carex pennsylvanica - Sporobolus cryptandrus Cyperus schwentzeii - Calamovilfa longifolia community on active sand in the Manito Sandhills of west-central Saskatchewan. This community type was found on sparsely vegetated, active east/west oriented sand dunes that were rapidly drained. The soils were coarse-textured and had little to no organic matter to retain moisture.

The Montana Natural Heritage Program (2002) listed a Sporobolus cryptandrus Shrub Herbaceous Vegetation association as a natural plant community for the state. The community was ranked G2/S2 for Montana, but unfortunately, no description of the community was provided.

The State of Idaho also listed several natural Sporobolus cryptandrus plant communities, including:

> Sporobolus cryptandrus - Poa secunda Medium-tall bunch temperate or subpolar grassland,

> Sporobolus cryptandrus Medium-tall temperate or subpolar grassland with a needle-leaved or microphyllous evergreen shrub layer and

D Heterotheca villosa / Sporobolus cryptandrus Low temperate or subpolar forb vegetation.

Natureserve (2002) listed two Alliances and one Association with Sporobolus cryptandrus as the dominant species. A Sporobolus cryptandrus Herbaceous Alliance was described for the lower Salmon and Snake river canyons of Idaho, Oregon and Washington, the Columbia River in central Washington and the Green and Virgin rivers in Utah (Natureserve 2002). This alliance typically occurred on river terraces, lower slopes of benches and alluvial fans. The elevation ranges from $240-1460 \mathrm{~m}$ and sites are flat to gently sloping (up to $30 \%$ ) and tend to occur on all aspects. The climate in the canyon bottoms was relatively hot and dry and soils were moderately deep and derived from loess and alluvium-colluvium. The soil texture varies from sandy loam to silt loam.

A Sporobolus cryptandrus - Poa secunda Herbaceous Vegetation association was described by Natureserve (2002) for the Columbia Basin and lower Snake River, where it occurred on gentle, lower slopes. It was also found on river terraces in the valleys of the Snake and Clearwater rivers. Sites were typically dominated by Sporobolus cryptandrus, although Poa secunda was common but varied in abundance. Aristida purpurea var. longiseta (= Aristida longiseta) and Hesperostipa comata (= Stipa comata) were also frequently present in low abundance.

A Sporobolus cryptandrus Shrub Herbaceous Alliance was described from Montana, Idaho and New Mexico (Natureserve 2002). In New Mexico, the alliance occurred in the northwestern part of the state on alluvial flats at an elevation of approximately $2140 \mathrm{~m}$. The climate was semi-arid with most of the highly variable annual precipitation falling during the summer as high-intensity storms. Sites are nearly level, soils are calcareous, loamy and generally less than $25 \mathrm{~cm}$ deep. 


\subsubsection{Artemisia cana / Stipa comata Association}

Silver sagebrush / needle and thread

A similar community type has been described in several sources for Alberta. Holcroft Weerstra (2001) describe an Artemisia cana / Stipa comata community that was found to occur along old river terraces, badlands, ravine side slopes and valley walls on a range of parent materials, but occurred most often on sandy glacial drift and alluvium. Adams et al. (1997) also described an Artemisia cana / Calamovilfa longifolia - Stipa comata from CFB-Suffield in southeastern Alberta. This community was typically found on glaciofluvial channel banks, with slopes greater than $15 \%$ and also on glaciofluvial terraces, with superimposed sand dunes. On both parent materials, the soil texture was sand to loamy sand. ANHIC currently lists an Artemisia cana / Stipa comata Shrub Herbaceous community and an Artemisia cana / Stipa comata - Calamovilfa longifolia Shrub Herbaceous community, both of which are currently ranked S2S3.

Coupland (1950) described a Rosa woodsii (Artemesia cana / Elaeagnus commutata) community type for the prairie provinces that was found in undulating to gently rolling areas between stabilized dunes. At these locations, the water table was typically within eight to twelve feet of the soil surface. However, the author did not describe the associated graminoid composition.

Cooper et al. (2001) described an Artemisia cana / Hesperostipa comata Shrub Herbaceous Vegetation association in the Bitter Creek / Frenchman Creek area in Montana. It was considered to be a minor type, due to limited distribution of coarse textured materials. At this location, the community type was found on benches to gently inclined slopes (30\% maximum recorded) in the vicinity of breaklands. This community type was found to occur on a variety of parent materials but was located dominantly on well-drained, often sandy glacial drift and sandy alluvium. The Montana Natural Heritage Program (2002) lists an Artemisia cana / Stipa comata Shrub Herbaceous Vegetation natural plant community for Montana. It is currently ranked S3 for the state.

Faber-Langendoen (2001) described an Artemisia cana / Hesperostipa comata Shrub Herbaceous Vegetation from the Midwestern United States. The author considered this community to be a small patch type, with a narrow geographic distribution, though it may be expected to occur in Saskatchewan and North Dakota. The author considered habitats with the potential to support this type to be relatively abundant, but the type itself to be comparatively uncommon. The community was typically found on well-drained benches and gently inclined landforms in a primarily agricultural landscape and thus puts it at a moderate risk for agriculture conversion. Fortunately this landform also occurred in breakland and badland environments that are less attractive for agriculture, thus reducing the risk of this type being converted to agriculture.

Natureserve (2002) reported an Artemisia cana / Hesperostipa comata Shrub Herbaceous Vegetation community occurring in the northwestern Great Plains. This shrub prairie association generally occurred in small patches (less than 1 hectare). Sites occurred on various parent materials, but mostly well-drained, often sandy, glacial drift and sandy alluvium. Artemisia cana was the dominant shrub with canopy coverages to $50 \%$, but averaged around $25 \%$. 


\subsection{Assignment of a Preliminary Provincial Ranking and Identification of Knowledge Gaps}

The amount (i.e. proposed plant communities with only one plot) and quality of available data used to describe the proposed sand dune and sand plain community types and assign provincial rankings differed from community to community. Furthermore, mapping of community types was not a component of this project. As such, there is no spatial context to provide an estimate of how much area each community type covers. Consequently, some community types were difficult to rank due to deficient or incomplete data and a lack of spatial context. For those communities that are ranked, more information is required to confirm the proposed rank. The recommended provincial ranks for each of the proposed community types is presented below, in association with an explanation of information gaps and recommended strategies to help address these gaps.

\subsubsection{Populus deltoides / Glycyrrhiza lepidota - Juncus balticus Association Western cottonwood / Wild licorice - wire rush}

\section{Preliminary Rank: S2S3}

A review of floristically similar plant communities throughout the Great Plains region revealed that this community type had been documented only in fluvial zones within Alberta, but in a similar sand dune/plain habitat in the Great Sand Hills of Saskatchewan. A somewhat similar community has been reported in numerous states, although restricted primarily to a fluvial-dominated habitat. Populus deltoides is currently ranked S3 by the province and Natureserve ranks a somewhat similar community type as G3G4. A preliminary rank of S2S3 is suggested for Alberta, due to the limited number of reports of this community type in a non-fluvial environment and also due to the current ranking of $P$. deltoides as $\mathrm{S} 3$.

This community was found in localized depressions between sand dunes and in more open sand plain areas. It was observed in a number of locations in the Pakowki Sandhill area and in certain locales, it appeared to be co-dominated by Populus tremuloides. One plot indicated that Populus tremuloides was a co-dominant species, although it shared a high prominence of understorey species that were also found in the Populus deltoides dominated plots. With limited plot data, it was difficult to assign a new community type based solely on one plot. As such it was included with this community type, but may indeed represent a different community type. Additional surveys of the Pakowki and other mixedgrass sandhill areas would be advantageous to identify this potential type. 


\subsubsection{Rosa woodsii / Sporobolus cryptandrus Association Common wild rose / sand dropseed}

\section{Preliminary Rank: S3?}

Despite Rosa woodsii being a widely distributed and often abundant species, little information could be found documenting Rosa woodsii dominated plant community types. A prior survey of the Pakowki Sandhills (Komex 1993) noted its presence and a survey of the Great Sand Dunes mentioned that Rosa woodsii dominated communities could be dominant in leeward areas where sand accumulation was occurring (Epp and Townley-Smith 1980). It was also listed as a community type in two locations for the state of Montana, where it is ranked G5/S5 and is a reported plant community type for Idaho. Unfortunately no floristic or habitat descriptions accompany these records.

Considering that Sporobolus cryptandrus is currently ranked S3 by the province of Alberta and its limited documentation as a mixedgrass or sand hill community in the literature, a preliminary rank of S3? is recommended until additional information regarding this potential community type becomes available.

\subsubsection{Salix amygdaloides - Rosa woodsii / Juncus balticus - Sporobolus cryptandrus Association \\ Peach-leaf willow - common wild rose / wire rush - sand dropseed}

\section{Preliminary Rank: S3?}

Little information could be found documenting Salix amygdaloides dominated plant community types within Alberta. A prior survey of the Pakowki Sandhills (Komex 1993) noted its presence as both a shrub and a tree, in low-lying areas and a survey of CFB Suffield noted a general Salix spp. / Stipa comata association along the floodplains and terraces of the South Saskatchewan River (Jaques 1977). Macdonald (1996) noted that Salix amygdaloides was an infrequent component in these wetter habitat types. Documentation of Salix amygdaloides or Salix amygdaloides / Salix exigua Woodlands along riparian zones exists for a number of Great Plains and Northern Rocky Mountain states. However, the habitat of these communities differs somewhat, in that periodic flooding or soil saturation is considered to be a natural factor in the riparian-type Salix amygdaloides-dominated communities.

Considering that Salix amygdaloides and Sporobolus cryptandrus are currently ranked S3 by the province of Alberta and its limited documentation as a mixedgrass or sandhill community in the literature, a preliminary rank of S3? is recommended until additional information regarding this potential community type becomes available. 


\subsubsection{Elaeagnus commutata / Glycyrrhiza lepidota Association Silverberry / wild licorice}

\section{Preliminary Rank: S2}

A survey of Dillberry Provincial Park reported a meadow Elaeagnus commutata community with Glycyrrhiza lepidota and Juncus balticus and Calamovilfa longifolia. Wheatley and Bentz (2002) suggested a preliminary ranking of S2 for this community type. An Elaeagnus commutata / Symphoricarpos occidentalis - Rosa woodsii / Poa palustris was also described from the Central Parkland occurring near Wainwright on subxeric to submesic dune sites with good internal soil drainage (Fehr 1984), which was ranked SU (Wheatley and Bentz 2002). Currently, ANHIC lists an Elaeagnus commutata / Pascopyrum smithii Shrubland community for the Grassland Natural Region and it is ranked S3 (Allen 2002).

A preliminary rank of S2 is suggested for this community type, as it shares a similar floristic composition as the community type reported for Dillberry Provincial Park. Although it was not found in the immediate vicinity of a lake, it was found in depressional, moisture-receiving locations between sand dunes.

\subsubsection{Elaeagnus commutata / Artemisia ludoviciana - Calamovilfa longifolia Association}

Silverberry / Prairie sagewort - sand grass

\section{Preliminary Rank: S2?}

A survey of Dillberry Provincial Park reported a meadow Elaeagnus commutata community with Glycyrrhiza lepidota and Juncus balticus and Calamovilfa longifolia. Wheatley and Bentz (2002) suggested a preliminary ranking of S2 for this community type. An Elaeagnus commutata / Symphoricarpos occidentalis - Rosa woodsii / Poa palustris community was also described from Central Parkland occurring near Wainwright on subxeric to submesic dune sites with good internal soil drainage (Fehr 1984), which was ranked SU (Wheatley and Bentz 2002). Currently, ANHIC lists an Elaeagnus commutata / Pascopyrum smithii Shrubland community for the Grassland Natural Region and it is ranked S3 (Allen 2002).

A preliminary rank of S2? is suggested for this community type, as there is only one sample plot from the Pakowki Sandhills area and it shares some floristic and environmental characteristics with the community type described above. Further sampling in the Pakowki Sandhills and other sandhill and sand plains areas could help to clarify the status of this community type. 


\subsubsection{Prunus virginiana / Calamovilfa longifolia Association Choke cherry - sand grass}

\section{Preliminary Rank: S4}

A prior survey of the Pakowki Sandhills (Komex 1993) noted dense Prunus virginiana thickets along low dune ridges and slopes, but did not provide a further description of this type. In Montana, a Prunus virginiana Shrubland was found as a small patch community within the Medicine Lake Sandhills (Heidel et al. 2000) although elsewhere in the state it is considered a riparian community. Montana and Natureserve (MNHP 2002; Natureserve 2002) currently rank this community type as G4Q/S4. In Alberta, Prunus virginiana is currently ranked as S5 while Calamovilfa longifolia is ranked S4. As such, a preliminary rank of S4 is recommended for the Prunus virginiana / Calamovilfa longifolia community type.

\subsubsection{Eurotia lanata / Stipa comata - Calamovilfa longifolia Association Winter fat / needle and thread - sand grass}

\section{Preliminary Rank: S4?}

Little information could be found documenting Eurotia lanata dominated plant community types within Alberta or Saskatchewan. A prior survey of the Pakowki Sandhills (Komex 1993) noted its presence in association with Bouteloua gracilis - Stipa comata on relatively level sites. Looman (1980) also noted that is a component of a Stipa comata grassland association. Several descriptions of a Eurotia lanata dominated plant community type exist for the Great Plains and Midwestern regions of the United States. Both Montana (MNHP 2002) and Natureserve (2002) report a Krascheninnikovia lanata / Hesperostipa comata dwarf shrubland association, which is ranked as G3/S3. Currently ANHIC ranks Eurotia lanata and Calamovilfa longifolia as S4, while Stipa comata is ranked S5. Therefore, due to the lack of information regarding this community type in Alberta and the individual rankings for each of the component species, a preliminary rank of $\mathrm{S} 4$ ? is recommended for this community type.

\subsubsection{Salix exigua / Glycyrrhiza lepidota - Juncus balticus Association}

Sandbar willow / wild licorice - wire rush

\section{Preliminary Rank: SU}

No information related to a Salix exigua dominated shrubland could be found from within Canada. Several Salix exigua dominated community types from the Great Plains and Midwestern regions of the United States were found, but in most instances the community types were associated with riparian habitats and experienced seasonally or temporary flooding. ANHIC currently ranks Salix exigua and Juncus balticus as S5 while 
Glycyrrhiza lepidota is ranked as S4. Despite the relative widespread distribution of the dominant species, little is recorded relating this community type to sandhill and sand plain habitats. As such a preliminary rank of SU is recommended while further studies are conducted to clarify the status of this community type.

\subsubsection{Glycyrrhiza lepidota - Calamovilfa longifolia Association \\ Wild licorice - sand grass}

\section{Preliminary Rank: SU}

Very little information exists in either Canada or the United States regarding Glycyrrhiza lepidota dominated community types. A prior survey of the Pakowki Sandhills (Komex 1993) noted Glycyrrhiza lepidota occurring with Calamovilfa longifolia and Artemisia ludoviciana. The Montana Natural Heritage Program (2002) notes a Glycyrrhiza lepidota herbaceous vegetation association as a natural plant community for the state, but provides a ranking of S? Consequently, a preliminary rank of SU is recommended while further studies are conducted to clarify the status of this community type.

\subsubsection{Glycyrrhiza lepidota - Artemisia spp. - Stipa comata Association Wild licorice - sage - needle and thread}

\section{Preliminary Rank: SU}

Very little information exists in either Canada or the United States regarding Glycyrrhiza lepidota dominated community types. A prior survey of the Pakowki Sandhills (Komex 1993) noted Glycyrrhiza lepidota occurring with Calamovilfa longifolia and Artemisia ludoviciana. The Montana Natural Heritage Program (2002) notes a Glycyrrhiza lepidota herbaceous vegetation association as a natural plant community for the state, but provides a ranking of S?. Consequently, a preliminary rank of SU is recommended while further studies are conducted to clarify the status of this community type.

\subsubsection{Rumex venosus Association Wild begonia}

\section{Preliminary Rank: SU}

Very little information exists in either Canada or the United States regarding Rumex venosus dominated community types. No references to any community types could be found in Alberta, although Looman (1980) notes a Rumex venosus Alliance for the Canadian Prairie Provinces, where it tends to occur on highly mobile sand dunes. Epp and Townley-Smith (1980) also noted that Rumex venosus could be prominent on active sand dunes in Saskatchewan, though it is not described as a community type per-se. Consequently, a preliminary rank of SU is recommended while further studies are conducted to clarify the status of this community type. 


\subsubsection{Oryzopsis hymenoides - Sporobolus cryptandrus Association Indian rice grass - sand dropseed Association}

\section{Preliminary Rank: S3}

Very little information exists in Alberta or other prairie provinces regarding Oryzopsis hymenoides - Sporobolus cryptandrus communities. One report exists of a Carex foenea - Calamovilfa longifolia - Elymus Canadensis - Oryzopsis hymenoides community from an active blowout in the Wainwright sand dune area. However, the species present in this dune community are rather different than those found in the Pakowki Sandhills. Several surveys from Saskatchewan report a Psoralea lanceolata - Oryzopsis hymenoides community type in active sand dunes, but not an Achnatherum hymenoides - Sporobolus cryptandrus community type. In the United States, the Oryzopsis hymenoides Psoralidium lanceolata community type has been described for Montana (Heidel et al. 2000) and is ranked G3Q / S? by the Montana Natural Heritage Program (2002). Natureserve (2002) also reports an Achnatherum hymenoides Herbaceous Alliance that is found on active sand dunes throughout the Great Plains and upland plateaus, but alliances are not given conservation ratings.

ANHIC currently ranks Oryzopsis hymenoides as S3S4 and Sporobolus cryptandrus as S3. Therefore, due to the limited area in which this community type was found, limited reports of similar community types and the component species current rankings, a preliminary ranking of $\mathrm{S} 3$ is recommended for this community type.

\subsubsection{Stipa comata - Oryzopsis hymenoides Association \\ Needle and thread - Indian rice grass}

\section{Preliminary Rank: S2S3}

Very little information exists in Alberta or other prairie provinces regarding Stipa comata - Oryzopsis hymenoides communities. One report exists of a Carex foenea-Calamovilfa longifolia - Elymus Canadensis - Oryzopsis hymenoides community from an active blowout in the Wainwright sand dune area. However, the species present in this dune community are rather different than those found in the Pakowki Sandhills. Several surveys in Saskatchewan report Stipa comata dominated communities on both upland prairie and sand dune habitats, but not in association with Oryzopsis hymenoides. Natureserve (2002) reports two potentially related community types. One is an Hesperostipa comata - Achnatherum hymenoides association that is ranked G2 but has not been found outside the Great Divide Basin in Wyoming. The other similar type listed is an Hesperostipa comata Bunch Herbaceous Vegetation Alliance, from the Wyoming Basin, Colorado Plateau, Great Basin and Columbia Plateau. However, this alliance is not rated and it is also noted to not occur on dunes.

ANHIC currently ranks Oryzopsis hymenoides as S3S4 and Stipa comata S5. However, based on its similarities with the similar reported association in Natureserve (2002) and 
the ANHIC ranking for Oryzopsis hymenoides, a preliminary ranking of $\mathrm{S} 2 \mathrm{~S} 3$ is suggested for this community type.

\subsubsection{Artemisia spp. - Stipa comata - Calamovilfa longifolia Association}

Sage - needle and thread - sand grass

\section{Preliminary Rank: S3S4}

Several similar community types have been reported for Alberta and Saskatchewan, although they vary somewhat in species composition and habitat. Smoliak (1984) reports two related community types, one on sandy upland prairie and the other on moist, coulee bottoms in the Manyberries region. Adams et al. (1997) reported a similar community type, though lacking the Artemisia spp. on coarse textured fluvial and glaciofluvial deposits in CFB-Suffield. Hulett et al. (1966) also report a community on partially stabilized dunes with a similar floristic composition.

Natureserve (2002) reports a related association, however this community type places Calamovilfa longifolia as the dominant grass. Furthermore, although Artemisia spp. are noted to be present in certain habitats, they are not considered to be a diagnostic species in the association. This association is ranked as G3. ANHIC ranks Hesperostipa comata as S5 and Calamovilfa longifolia as S4. Based on the documentation of this community type and similar reported types, a preliminary ranking of S3S4 is suggested for the Artemisia spp. - Hesperostipa comata-Calamovilfa longifolia community type.

\subsubsection{Stipa comata - Cyperus schweinitzii - Calamovilfa longifolia Association Needle and thread - sand nut-grass - sand grass}

\section{Preliminary Rank: S2?}

Several similar community types have been reported for Alberta and Saskatchewan, although they vary somewhat in species composition and habitat. Smoliak (1984) reports two related community types, one on sandy upland prairie and the other on moist, coulee bottoms in the Manyberries region. Adams et al. (1997) reported a similar community type, on coarse textured fluvial and glaciofluvial deposits in CFB-Suffield. Hulett et al. (1966) also report a community on partially stabilized dunes with a similar floristic composition.

Natureserve (2002) reports a related association, however this community type places Calamovilfa longifolia as the dominant grass and does not mention the presence of Cyperus schweinitzii. This association is ranked as G3. ANHIC ranks Stipa comata as S5, Cyperus schweinitzii as S2 and Calamovilfa longifolia as S4. Based on the documentation of this community type and similar reported types, a preliminary ranking of S2? is suggested for the Stipa comata - Cyperus schweinitzii - Calamovilfa longifolia community type. 
3.4.16 Sporobolus cryptandrus - Calamovilfa longifolia - Oryzopsis hymenoides Association

Sand dropseed - sand grass - Indian rice grass

\section{Preliminary Rank: S2S3}

Several similar community types have been reported for Alberta and Saskatchewan, although they vary somewhat in species composition and habitat. Wheatley and Bentz (2002) and Meijer and Karpuk (1999) report a related Sporobolus cryptandrus Calamovilfa longifolia - Koeleria macrantha-Carex obtusata community type from the Central Parkland and it was assigned a preliminary rank of S3. Reports of similar community types by Looman (1980) and Thorpe and Godwin (1993) also exist from Saskatchewan. Natureserve (2002) also reports several related Sporobolus cryptandrus dominated Herbaceous Alliances, although the habitat varies somewhat. Unfortunately, the alliances are not provided with a conservation ranking.

Based on previous reported rankings for similar communities (ANHIC S2 for a Sporobolus cryptandrus active dune community) and ANHIC's S3 ranking of Sporobolus cryptandrus, a preliminary ranking of S2S3 is suggested for the Sporobolus cryptandrus - Calamovilfa longifolia - Oryzopsis hymenoides community type.

\subsubsection{Artemisia cana / Stipa comata Association \\ Silver sagebrush / needle and thread}

\section{Preliminary Rank: S2S3}

This community is currently tracked by ANHIC for the Grassland Natural Region and is ranked S2S3. The distribution and area of the Artemisia cana / Stipa comata community in the Pakowki Sandhills was rather limited and thus does not justify altering the ranking based on an increase in reported area. 


\subsection{Conclusion}

The preliminary classification of the Pakowki Sandhills communities based on the analysis of plot data and a comparison with community types described in existing literature, revealed 17 community types. Community types included all classes, except Non-Vascular and Sparse. All proposed community types were described and assigned a suggested preliminary ranking. Cross-reference tables were created to present similar communities described in the literature, rate their similarity with the Pakowki Sandhills communities and explain their relation to the proposed community types. Knowledge gaps were identified and strategies to address these gaps were provided where possible.

Several difficulties were encountered which should be noted. In the literature, community type descriptions vary from a single sentence to extremely detailed descriptions and in most instances, there is insufficient information to adequately describe community structure and assign a conservation rank (i.e. based on distribution and abundance). No quantification of area for community types could be found in the literature, aside from qualitative notes such as 'widely distributed' or 'abundant.' Furthermore, no quantification of the area of community types was required for this project. As such, determining the areal coverage of community types was done through observation and estimation. Mapping of community types at a relatively detailed scale would provide a greater confidence behind the estimation of conservation ranks for this project and provide a means to track impacts on community types in the future.

The information in this report can be used to update the community-tracking list by including new community types. Finally, this report can also be used to decide which community types require further studies and to prioritize these studies. 


\subsection{Literature Cited}

Achuff, P.L. 1994. Natural regions, Subregions and Natural History Themes of Alberta. Prepared for Parks Service, Alberta Environmental Protection, Edmonton, Alberta.

Adams, G.D., G.C.Trottier, W.L. Strong, I.D. MacDonald, S.J. Barry, P.G. Gregoire, G.W. Babish and G. Weiss. 1997. Vegetation Component Report: Canadian Forces Base Suffield National Wildlife Area Wildlife Inventory. Canadian Wildlife Service, Environment Canada, Prairie and Northern Region. Edmonton, Alberta.

Alberta Environmental Protection. 1993. Alberta plants and fungi - master species list and species group checklists. Pub. No.: Ref. 75. Edmonton, Alberta.

Alberta Environmental Protection. 1994. Ecological land survey site description manual. Unpubl. Rep. Edmonton, Alberta. 165pp.

Alberta Environmental Protection 1997. The Grassland Natural Region of Alberta. Natural Resources Service, Recreation and Protected Areas Division, Natural Heritage Protection and Education Branch. Edmonton, Alberta.

Allen, L. 2002. Alberta Natural Heritage Information Centre Preliminary Plant Community Tracking List, Alberta Community Development, Edmonton, Alberta.

Barker, W.T. and W.C. Whitman. 1988. Vegetation of the Northern Great Plains. Rangelands 10(6) 266-272.

Bowers, J.E. The plant ecology of inland dunes in western North America.

Braun-Blanquet, J. 1965. Plant sociology : the study of plant communities. Authorized English translation of Pflanzensoziologie by J. Braun-Blanquet. Translated, rev. and ed. by George D. Fuller and Henry S. Conard.Hafner Publishing Co., Longon, England. 439 pp.

Burgess, R.L. 1965. A study of plant succession in the sandhills of southeastern North Dakota. Proceedings of the North Dakota Academy of Science 19:62-80.

Chadwick, H.W. and P.D. Dalke. 1965. Plant succession on dune sands in Fremont County, Idaho. Ecology 46(6): 765-780.

Cooper, S.V., C. Jean and B.L. Heidel. 1999. Plant associations and Related Botanical Inventory of the Beaverhead Mountains Section, Montana. Unpublished report to the Bureau of Land Management. Montana Natural Heritage Program, Helena. 235 pp.

Cooper, S.V., C. Jean and P. Hendricks. 2001. Biological Survey of a Prairie Landscape in Montana's Glaciated Plains. Report to the Bureau of Land Management, Montana 
Natural Heritage Program, Helena Montana. 24pp. plus appendices.

Corns, I.G.W. 1983. Forest community types of west-central Alberta in relation to selected environmental factors. Canadian Journal of Forest Research 13:995-1010.

Coupland, R.T. 1950. Ecology of mixed prairie in Canada. Ecological Monographs 20:273-315.

Coupland, R.T. 1960. A reconsideration of grassland classification in the northern great plains of North America. Journal of Ecology 49:135-167.

Dowding, E.S. 1929. The vegetation of Alberta: III The sandhill areas of central Alberta, with particular reference to the ecology of Arceuthobium americanum. Journal of Ecology, Vol. 17: 82-105.

Epp, H.T. and L. Townley-Smith. 1980. The great sand hills of Saskatchewan. Saskatchewan Deportment of the Environment, Policy, Planning and Research Branch. Regina, Saskatchewan.

Faber-Langendoen, D. editor. 2001. Plant communities of the Midwest: Classification in an ecological context. Association for Biodiversity Information, Arlington, VA. 61 pp. + appendix (705 pp.).

Fehr, A.W. 1984. Wainwright study area: A biophysical inventory. Natural Areas Program, Alberta Energy and Natural Resources. Natural Areas Technical Report No. 15. Edmonton, Alberta.

Grossman, D.H., K.L. Goodin and C.L. Reuss, editors. 1994. Rare plant communities of the conterminous United States: an initial survey. The Nature Conservancy, Arlington, Virginia, USA.

Grossman, D.H., D. Faber-Langendoen, A.S. Weakley, M. Anderson, P. Bourgeron, R. Crawford, K. Goodin, S. Laandal, K. Metzler, K.D. Patterson, M. Pyne, M. Reid and L. Sneddon. 1998. International classification of ecological communities: terrestrial vegetation of the United States, Volume 1. The National Vegetation Classification System: development, status and applications. The Nature Conservancy, Arlington, Virginia, USA.

Hanson, H.C. and W.C. Whitman. 1938. Grassland types in Western North Dakota. Ecological Monographs 8(1): 58-114.

Heidel, B, S.V. Cooper and C. Jean. 2000. Plant species of special concern and plant associations of Sheridan County, Montana. Report to U.S. Fish and Wildlife Service. Montana Natural Heritage Program, Helena, Montana. 22pp. plus appendices.

Hill, M.O. and H.G. Gauch. 1980. Detrended correspondence analysis: an improved 
ordination technique. Vegetatio 42:47-58.

Holcroft-Weerstra, A.C. 2001. Preliminary Classification of Silver Sagebrush (Artemesia cana) Community Types. Alberta Natural Heritage Information Centre, Edmonton, Alberta. Biota Consultants, Cochrane, Alberta.

Hulett, G.K., R.T. Coupland, R.L. Dix. 1966. The vegetation of dun sand areas within the grassland region of Saskatchewan. Canadian Journal of Botany 44: 1307-1331.

Jaques, D.R. 1977. The vegetation and effects of grazing on the eastern portion of the Suffield Military Reserve, Alberta. pp. D41-D190-. In Stelfox, J.G. ed. Effects of livestock grazing on mixed prairie range and wildlife in PFRA pastures. Suffield Military Reserve. Range-Wildlife Study Committee, Can. Wild. Ser. Rep. Edmonton, Alberta.

Jones, G.P. 1998a. Ecological evaluation of the potential Cheyenne River Research Natural Area within the Thunder Basin National Grassland, Converse County, Wyoming. Prepared for Nebraska, National Forest, and USDA Forest Service. Laramie, Wyoming, USA.

Jones, G.P. 1998b. Ecological evaluation of the potential Antelope Creek Research Natural Area within the Thunder Basin National Grassland, Converse County, Wyoming. Prepared for Nebraska, National Forest, and USDA Forest Service. Laramie, Wyoming, USA.

Kartesz, JT. 1999. A synonymized checklist and atlas with biological attributes for the vascular flora of the United States, Canada and Greenland. First edition. In: Kartesz, JT and CA Meacham. Synthesis of the North American flora [computer program]. Version 1.0. North Carolina Botanical Garden: Chapel Hill, NC.

Komex International Ltd. 1993. Preliminary report on biophysical inventory of the Pakowki Sandhills sensitive area. Land Information Services Division, Alberta Forestry Lands and Wildlife, Edmonton, Alberta.

Looman, J. 1980. The vegetation of the Canadian Prairie Provinces II. The grasslands, Part I. Phytocoenologia 8(2) 153-190.

Macdonald, I.D. 1996. Vascular Plant Flora of Canadian Forces Base Suffield, National Wildlife Area. Canadian Wildlife Service, Environment Canada, Prairie and Northern Region, Edmonton, Alberta.

Marriott, H. J. and D. Faber-Langendoen. 2000. The Black Hills community inventory. Volume 2: Plant community descriptions. The Nature Conservancy, Midwest Conservation Science Center and Association for Biodiversity Information, Minneapolis, MN. 326 pp.

Meijer, M. and E. Karpuk. 1999. Dillberry Lake Provincial Park Biophysical Inventory. Natural Resources Service, Parkland Region, Alberta Environment, Edmonton, Alberta. 
Meclune, B. and M.J. Mefford. 1999. PC-ORD. Multivariate Analysis of Ecological Data, Version 4. MjM Software Design, Gleneden Beach, Oregon, USA.

Montana Natural Heritage Program. 2002. 2002 List of Ecological Communities for Montana. Montana Natural Heritage Program, Montana State Library, Helena, Montana.

Moss, E.H. 1983. Flora of Alberta. $2^{\text {nd }}$ Edition Revised by J.G. Packer. University of Toronto Press, Toronto, Ontario.

Natureserve Explorer: An online encyclopedia of life [web application]. 2001. Version 1.6. Arlington, Virginia, USA: Natureserve. Available:

http://www.natureserve.org/explorer. (Accessed: November 12, 2002 ).

Patriquin, D.L. and D. L. Skinner. 1992. Review and assessment of the vegetation, wildlife and habitat of CFB Suffield, Alberta. D.A. Westworth and Associates, Edmonton, Alberta.

Rust, S.K. 1997. Natural Plant Communities of Idaho: A Working List for the Conservation of Biological Diversity. Conservation Data Centre, Idaho Department of Fish and Game.

Schacht, W.H., J.D. Volesky, D. Bauer, A.J. Smart and E.M. Mousel. 2000. Plant community patterns on upland prairie in the eastern Nebraska sandhills. Prairie Naturalist 32(1):43-58. Jamestown, ND: Northern Prairie Wildlife Research Center Home Page. http://www.npwrc.usgs.ggov/resource/2001/plantcom/plantcom.htm (Version 20AUG2001).

Shetsen, I. 1987. Quaternary geology, southern Alberta. Alberta Research Council, Edmonton. Map.

Smoliak, S. 1985. Flora of the Manyberries research substation. Lethbridge Research Station Contribution, No. 6, Research Branch, Agriculture Canada. Lethbridge, Alberta.

Strong, W.L. 2002. Lodgepole pine / Labrador tea community types of western Canada. Canadian Journal of Botany 80:151-165.

Ter Braak, C.J.F. and I.C. Prentice. 1988. A theory of gradient analysis. Advances in Ecological Research 18:271-313.

Thorpe, J. and R. Godwin. 1993. Vegetation Survey of the Manito San Hills. Applied Plant Ecology Section, Saskatchewan Research Council. SRC Publication No. E-2550-1E-93.

United States Geological Survey. 2002a. Scotts Bluff National Monument USGS-NPS Vegetation Mapping Program Products-Vegetation Description. http:/biology/usgs.gov/npsveg/scbl/index.html

United States Geological Survey. 2002b. Devil's Tower National Monument USGS-NPS 
Vegetation Mapping Program Products-Vegetation Description.

http:/biology/usgs.gov/npsveg/deto/index.html

United States Geological Survey. 2002c. Agate Fossil Beds National Monument USGSNPS Vegetation Mapping Program Products-Vegetation Description.

http:/biology/usgs.gov/npsveg/agfo/index.html

United States Geological Survey. 2002d. Badlands National Park. USGS-NPS Vegetation Mapping Program Products-Vegetation Description.

http:/biology/usgs.gov/npsveg/badl/index.html

Wallis, C. and C. Wershler. 1988. Rare Wildlife and Plant Conservation Studies in Sandhill and Sand Plain Habitats of Southern Alberta. Alberta Forestry, Lands and Wildlife, Alberta Recreation and Parks. Edmonton, Alberta.

Wanek, W.J. and R.L. Burgess. 1965. Floristic composition of the sand prairies of southeastern North Dakota. Proceedings of the North Dakota Academy of Science 19:2640 .

Wheatley, M. and J. Bentz. 2002. A preliminary classification of plant communities in the Central Parkland Natural Subregion of Alberta. Alberta Sustainable Resource Development, Public Lands Division, Resource Data Branch, Edmonton, Alberta.

Whittaker, R.H. 1978. Ordination of Plant Communities. Dr. W. Junk Publishers, The Hague, Boston. 


Appendix 1. Glossary of Scientific and Common Plant Species Names 



\section{Scientific Name}

Acer negundo

Achillea millefolium

Achnatherum hymenoides

Agropyron dasystachyum

Agropyron fragile

Agropyron sibiricum

Agropyron smithii

Agropyron trachycaulum

Agrostis stolonifera

Ambrosia acanthicarpa

Ambrosia psilostachya

Antennaria microphylla

Antennaria parvifolia

Aristida purpurea var. longiseta

Artemisia campestris

Artemisia cana

Artemisia frigida

Artemisia ludoviciana

Artemisia tridentata ssp. wyomingensis

Artemisia spp.

Bouteloua gracilis

Bromus tectorum

Calamovilfa longifolia

Carex emoryi

Carex foenea

Carex lanuginosa

Carex obtusata

Carex pellita

Carex pensylvanica

Cenchrus longispinus

Cerastium arvense

Chamaesyce serpyllifolia

Chenopodium fremontii

Chenopodium pratericola

Chenopodium subglabrum

Cirsium arvense

Cleome serrulata

Cornus sericea

Coryphantha vivipara

Cryptantha fendleri

Cyperus schweinitzii

Descurainia sophia

Echinacea angustifolia

Elaeagnus angustifolia

Elaeagnus commutata

Elymus canadensis

Elymus lanceolatus ssp. lanceolatus

Equisetum arvense

Equisetum hyemale

Erigeron caespitosus

Escobaria vivipara

Euphorbia esula

Eurotia lanata

\section{Common Name}

Manitoba maple

common yarrow

Indian rice grass

northern wheat grass

Siberian wheat grass

Siberian wheat grass

western wheat grass

slender wheat grass

creeping bentgrass

bur ragweed

perennial ragweed

little-leaf pussytoes

small-leave pussytoes

Fendler threeawn

field sagewort

silver sagebrush

pasture sagewort

prairie sagewort

Wyoming big sagebrush

sage

blue grama

cheatgrass

sand grass

Emory's sedge

dryspike sedge

wooly sedge

blunt sedge

wooly sedge

sunloving sedge

mat sandbur

field chickweed

thymeleaf sandmat

Fremont's goosefoot

desert goosefoot

smooth narrow-leaved goosefoot

Canada thistle

bee plant

redosier dogwood

cushion cactus

Fendler's cryptanth

sand nut-grass

flixweed

blacksamson echinacea

Russian olive

silver-berry

Canada wild rye

northern wheat grass

common horsetail

scouring rush

tufted fleabane

cushion cactus

leafy spurge

winter fat 


\section{Scientific Name}

Franseria acanthicarpa

Fraxinus pennsylvanica

Glycyrrhiza lepidota

Grindelia squarrosa

Helianthus annuus

Helianthus couplandii

Helianthus pauciflorus ssp. subrhomboideus

Helianthus petiolaris

Helianthus subrhomboideus

Hesperostipa comata

Heterotheca villosa

Juncus balticus

Kochia scoparia

Koeleria macrantha

Krascheninnikovia lanata

Lactuca pulchella

Lactuca tartarica var. pulchella

Lepidium densiflorum

Leymus canadensis

Liatris punctata

Lygodesmia rostrata

Maianthemum stellata

Melilotus spp.

Mentha arvensis

Oenothera nuttallii

Opuntia macrorhiza

Opuntia polyacantha

Oryzopsis hymenoides

Pascopyrum smithii

Phalaris arundinacaea

Phyla lanceolata

Plantago patagonica

Poa palustris

Poa pratensis

Poa secunda

Populus angustifolia

Populus deltoides

Populus fremontii

Populus tremuloides

Potentilla pensylvanica

Prunus americana

Prunus virginiana

Psoralea lanceolata

Psoralidium lanceolatum

Ribes oxyacanthoides

Rosa acicularis

Rosa woodsii

Rumex venosus

Salix amygdaloides

Salix bebbiana

Salix exigua

Salix interior

Salix lutea

\author{
Common Name \\ bur ragweed \\ green ash \\ wild licorice \\ curlycup gumweed \\ common annual sunflower \\ annual sunflower \\ rhombic-leaved sunflower \\ prairie sunflower \\ rhombic-leaved sunflower \\ needle-and-thread grass \\ golden aster \\ wire rush \\ Mexican-fireweed \\ June grass \\ winter fat \\ common blue lettuce \\ common blue lettuce \\ common peppergrass \\ Canada wild rye \\ dotted blazingstar \\ annual skeleton-weed \\ star-flowered Solomon's seal \\ sweet-clover \\ wild mint \\ white evening primrose \\ twistspine pricklypear \\ prickly pear \\ Indian rice grass \\ western wheat grass \\ reed canarygrass \\ lanceleaf fogfruit \\ wooly plantain \\ fowl bluegrass \\ Kentucky bluegrass \\ Sandberg bluegrass \\ narrow-leaf cottonwood \\ western cottonwood \\ Fremont cottonwood \\ aspen \\ Pennsylvania cinquefoil \\ American plum \\ chokecherry \\ lemon scurfpea \\ lemon scurfpea \\ wild gooseberry \\ prickly rose \\ common wild rose \\ wild begonia / veined dock \\ peachleaf willow \\ Bebb's willow \\ sandbar willow \\ sandbar willow \\ yellow willow
}




\section{Scientific Name}

Salix spp.

Salsola kali

Schizachyrium scoparium

Shinnersoseris rostrata

Smilacina stellata

Solidago canadensis

Solidago missouriensis

Spartina pectinata

Sporobolus cryptandrus

Stipa comata

Symphoricarpos occidentalis

Taraxacum officinale

Thermopsis rhombifolia

Tragopogon dubius

\author{
Common Name \\ willow \\ Russian thistle \\ little bluestem \\ annual skeleton-weed \\ star-flowered Solomon's seal \\ Canada goldenrod \\ Missouri goldenrod \\ prairie cord grass \\ sand dropseed \\ needle-and thread grass \\ buckbrush \\ common dandelion \\ golden bean \\ goat's beard
}




\begin{tabular}{|c|c|}
\hline Common Name & Scientific Name \\
\hline American plum & Prunus americana \\
\hline annual skeleton-weed & Lygodesmia rostrata \\
\hline annual skeleton-weed & Shinnersoseris rostrata \\
\hline annual sunflower & Helianthus couplandii \\
\hline aspen & Populus tremuloides \\
\hline Bebb's willow & Salix bebbiana \\
\hline bee plant & Cleome serrulata \\
\hline blacksamson echinacea & Echinacea angustifolia \\
\hline blue grama & Bouteloua gracilis \\
\hline blunt sedge & Carex obtusata \\
\hline buckbrush & Symphoricarpos occidentalis \\
\hline bur ragweed & Ambrosia acanthicarpa \\
\hline bur ragweed & Franseria acanthicarpa \\
\hline Canada goldenrod & Solidago canadensis \\
\hline Canada thistle & Cirsium arvense \\
\hline Canada wild rye & Elymus canadensis \\
\hline Canada wild rye & Leymus canadensis \\
\hline cheatgrass & Bromus tectorum \\
\hline chokecherry & Prunus virginiana \\
\hline common annual sunflower & Helianthus annuus \\
\hline common blue lettuce & Lactuca pulchella \\
\hline common blue lettuce & Lactuca tartarica var. pulchella \\
\hline common dandelion & Taraxacum officinale \\
\hline common horsetail & Equisetum arvense \\
\hline common peppergrass & Lepidium densiflorum \\
\hline common wild rose & Rosa woodsii \\
\hline common yarrow & Achillea millefolium \\
\hline creeping bentgrass & Agrostis stolonifera \\
\hline curlycup gumweed & Grindelia squarrosa \\
\hline cushion cactus & Coryphantha vivipara \\
\hline cushion cactus & Escobaria vivipara \\
\hline desert goosefoot & Chenopodium pratericola \\
\hline dotted blazingstar & Liatris punctata \\
\hline dryspike sedge & Carex foenea \\
\hline Emory's sedge & Carex emoryi \\
\hline Fendler threeawn & Aristida purpurea var. longiseta \\
\hline Fendler's cryptanth & Cryptantha fendleri \\
\hline field chickweed & Cerastium arvense \\
\hline field sagewort & Artemisia campestris \\
\hline flixweed & Descurainia sophia \\
\hline fowl bluegrass & Poa palustris \\
\hline Fremont cottonwood & Populus fremontii \\
\hline Fremont's goosefoot & Chenopodium fremontii \\
\hline goat's beard & Tragopogon dubius \\
\hline golden aster & Heterotheca villosa \\
\hline golden bean & Thermopsis rhombifolia \\
\hline green ash & Fraxinus pennsylvanica \\
\hline Indian rice grass & Achnatherum hymenoides \\
\hline Indian rice grass & Oryzopsis hymenoides \\
\hline June grass & Koeleria macrantha \\
\hline Kentucky bluegrass & Poa pratensis \\
\hline lanceleaf fogfruit & Phyla lanceolata \\
\hline
\end{tabular}




\section{Scientific Name}

Euphorbia esula

Psoralea lanceolata

Psoralidium lanceolatum

Schizachyrium scoparium

Antennaria microphylla

Acer negundo

Cenchrus longispinus

Kochia scoparia

Solidago missouriensis

Populus angustifolia

Stipa comata

Hesperostipa comata

Agropyron dasystachyum

Elymus lanceolatus ssp. lanceolatus

Artemisia frigida

Salix amygdaloides

Potentilla pensylvanica

Ambrosia psilostachya

Spartina pectinata

Artemisia ludoviciana

Helianthus petiolaris

Opuntia polyacantha

Rosa acicularis

Cornus sericea

Phalaris arundinacaea

Helianthus pauciflorus ssp. subrhomboideus

Helianthus subrhomboideus

Elaeagnus angustifolia

Salsola kali

Artemisia spp.

Sporobolus cryptandrus

Calamovilfa longifolia

Cyperus schweinitzii

Salix exigua

Salix interior

Poa secunda

Equisetum hyemale

Agropyron fragile

Agropyron sibiricum

Artemisia cana

Elaeagnus commutata

Agropyron trachycaulum

Antennaria parvifolia

Chenopodium subglabrum

Maianthemum stellata

Smilacina stellata

Carex pensylvanica

Melilotus spp.

Chamaesyce serpyllifolia

Erigeron caespitosus

Opuntia macrorhiza

Populus deltoides

Agropyron smithii 


\section{Common Name}

western wheat grass

white evening primrose

wild begonia / veined dock

wild gooseberry

wild licorice

wild mint

willow

winter fat

winter fat

wire rush

wooly plantain

wooly sedge

wooly sedge

Wyoming big sagebrush

yellow willow
Scientific Name

Pascopyrum smithii

Oenothera nuttallii

Rumex venosus

Ribes oxyacanthoides

Glycyrrhiza lepidota

Mentha arvensis

Salix spp.

Eurotia lanata

Krascheninnikovia lanata

Juncus balticus

Plantago patagonica

Carex lanuginosa

Carex pellita

Artemisia tridentata ssp. wyomingensis

Salix lutea 


Appendix 2. PC-ORD Output from Detrended Correspondence Analysis of Plot Data 


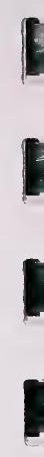

,

,

,

,

,

,

,

,

,

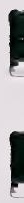

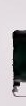

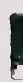

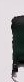




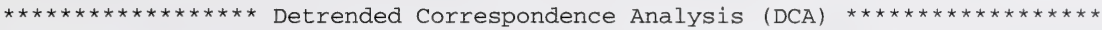

PC-ORD, Version 4.20

25 Nov $2002,13: 01$

SAND DUNE COMMUNITIES

Number of non-zero data items:

497

Downweighting selected. Weights applied to columns, in sequential order:

$\begin{array}{lllllllllll}0.615 & 0.300 & 0.300 & 0.300 & 0.300 & 0.565 & 0.565 & 0.300 & 0.300 & 0.300\end{array}$

$\begin{array}{lllllllllll}1.000 & 0.916 & 0.913 & 0.559 & 1.000 & 1.000 & 0.300 & 0.392 & 0.300 & 0.300\end{array}$

$\begin{array}{llllllllll}0.300 & 0.900 & 0.900 & 0.600 & 1.000 & 0.600 & 0.300 & 1.000 & 1.000 & 1.000\end{array}$

$\begin{array}{llllllllll}0.600 & 1.000 & 1.000 & 1.000 & 1.000 & 1.000 & 0.600 & 1.000 & 0.540 & 1.000\end{array}$

$\begin{array}{lllllllllll}1.000 & 1.000 & 0.300 & 0.663 & 0.700 & 1.000 & 1.000 & 0.300 & 0.800 & 1.000\end{array}$

$\begin{array}{llllllllll}0.600 & 0.300 & 0.426 & 1.000 & 1.000 & 1.000 & 1.000 & 0.300 & 0.900 & 0.833\end{array}$

1.0001 .0001 .0001 .0001 .000

Axes are rescaled

Number of segments: 30

Threshold: 0.00

Total variance ("inertia") in the species data: 6.2854

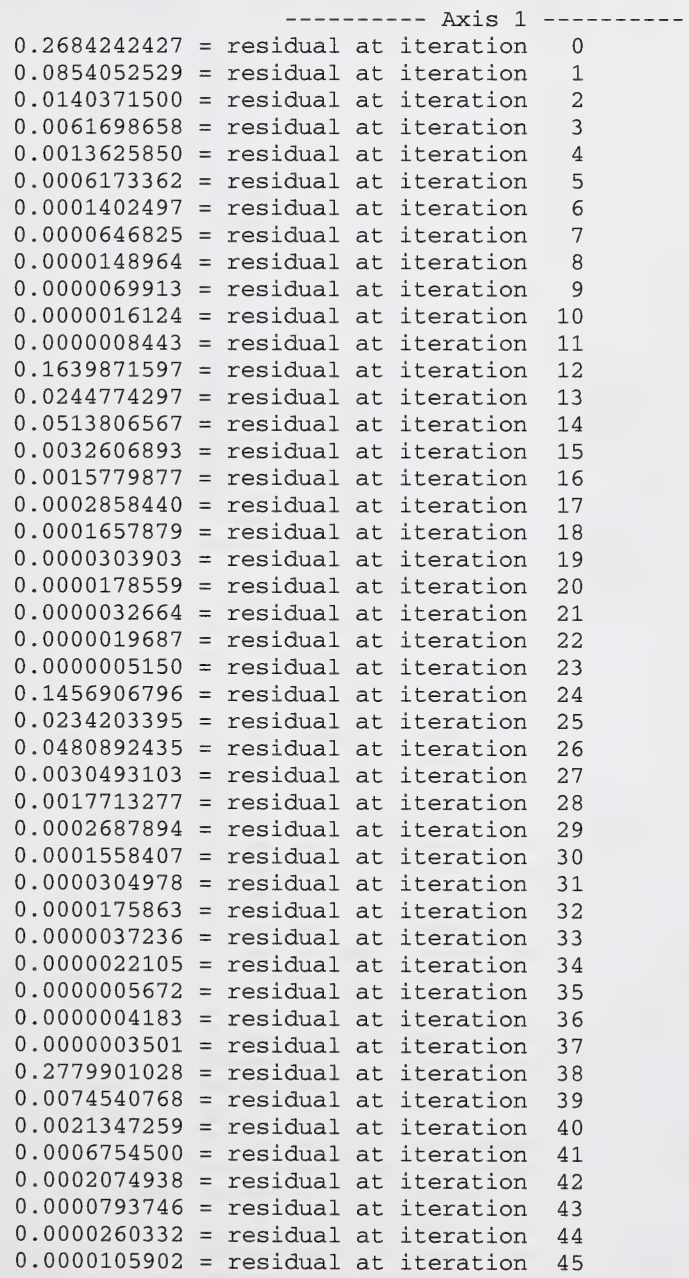


$0.0000036683=$ residual at iteration 46

0.0000015326 = residual at iteration 47

$0.0000005304=$ residual at iteration 48

0.0119315125 = residual at iteration 49

0.0029634687 = residual at iteration 50

0.0000002078 = residual at iteration 60

0.0000211076 = residual at iteration 70

0.0018477291 = residual at iteration 80

0.0139812361 = residual at iteration 90

$0.0000005091=$ residual at iteration 100

0.0000062506 = residual at iteration 110

$0.0000461937=$ residual at iteration 120

$0.0222807173=$ residual at iteration 130

0.1710713655 = residual at iteration 140

$0.0000009847=$ residual at iteration 150

0.0000099311 = residual at iteration 160

$0.0002753049=$ residual at iteration 170

0.0028829316 = residual at iteration 180

0.0192794092 = residual at iteration 190

0.0000006532 = residual at iteration 200

$0.0000043344=$ residual at iteration 210

0.0000274861 = residual at iteration 220

$0.0097351270=$ residual at iteration 230

0.0000010114 = residual at iteration 240

$0.0000127912=$ residual at iteration 250

0.0001706528 = residual at iteration 260

$0.0005976434=$ residual at iteration 270

$0.0020370230=$ residual at iteration 280

0.1597174108 = residual at iteration 290

$0.0000015046=$ residual at iteration 300

$0.0000147340=$ residual at iteration 310

$0.0000765337=$ residual at iteration 320

0.0011597162 = residual at iteration 330

$0.0019860768=$ residual at iteration 340

$0.2260046750=$ residual at iteration 350

0.0954641625 = residual at iteration 360

0.0000008501 = residual at iteration 370

$0.0003140370=$ residual at iteration 380

$0.0263471641=$ residual at iteration 390

0.1734091938 = residual at iteration 400

$0.0000005903=$ residual at iteration 410

0.0000467348 = residual at iteration 420

$0.0000296704=$ residual at iteration 430

$0.0007561108=$ residual at iteration 440

$0.0360311270=$ residual at iteration 450

0.0000008192 = residual at iteration 460

0.0002691617 = residual at iteration 470

0.0133251371 = residual at iteration 480

$0.0000009775=$ residual at iteration 490

0.0000034115 = residual at iteration 500

$0.0019896838=$ residual at iteration 510

$0.0212083757=$ residual at iteration 520

0.0000005526 = residual at iteration 530

$0.0000056518=$ residual at iteration 540

$0.0001769213=$ residual at iteration 550

$0.0478879064=$ residual at iteration 560

$0.0000007582=$ residual at iteration 570

0.0000120228 = residual at iteration 580

$0.0004150117=$ residual at iteration 590

$0.0002466936=$ residual at iteration 600

0.0018837127 = residual at iteration 610

0.2045992166 = residual at iteration 620

$0.0000096100=$ residual at iteration 630

$0.0000455938=$ residual at iteration 640

$0.0055995947=$ residual at iteration 650

0.1489204913 = residual at iteration 660

$0.1107899100=$ residual at iteration 670

$0.0000003468=$ residual at iteration 680

0.0000078266 = residual at iteration 690

$0.0004097962=$ residual at iteration 700

$0.0000001005=$ residual at iteration 710 
$0.0000026737=$ residual at iteration 720 $0.0000636807=$ residual at iteration 730 0.0006091066 = residual at iteration 740 $0.0165218879=$ residual at iteration 750 $0.0000096641=$ residual at iteration 760 $0.0000374746=$ residual at iteration 770 $0.0000017238=$ residual at iteration 780 0.0000040091 = residual at iteration 790 $0.0000317458=$ residual at iteration 800 $0.0000037804=$ residual at iteration 810 $0.0003344046=$ residual at iteration 820 $0.0202178955=$ residual at iteration 830 $0.0000006557=$ residual at iteration 840 $0.0001674164=$ residual at iteration 850 0.0006750197 = residual at iteration 860 $0.0000157669=$ residual at iteration 870 $0.0001539329=$ residual at iteration 880 $0.0045301383=$ residual at iteration 890 0.1807350367 = residual at iteration 900 $0.0000009182=$ residual at iteration 910 $0.0000329651=$ residual at iteration 920 $0.0000177802=$ residual at iteration 930 $0.0006978700=$ residual at iteration 940 0.0364268906 = residual at iteration 950 $0.0009799623=$ residual at iteration 960 $0.0008513225=$ residual at iteration 970 $0.1945322305=$ residual at iteration 980 $0.0000022841=$ residual at iteration 990

0.0534050353 = residual at iteration 999

$0.7860396504=$ eigenvalue

*** BEWARE *** RESIDUAL BIGGER THAN TOLERANCE, WHICH IS 0.0000001000

Length of gradient: Length of segments: Length of segments: Length of gradient:

$$
2.995
$$

Length of gradient: Length of segments: Length of segments: Length of segments: Length of gradient:

$$
\begin{array}{llllllllll}
0.32 & 0.32 & 0.31 & 0.30 & 0.29 & 0.29 & 0.26 & 0.19 & 0.13 & 0.11 \\
0.10 & 0.10 & 0.09 & 0.09 & 0.09 & & & & & \\
3.567 & & & & & & & &
\end{array}
$$$$
4.127
$$$$
\begin{array}{llllllllll}
0.24 & 0.24 & 0.22 & 0.20 & 0.19 & 0.20 & 0.21 & 0.21 & 0.21 & 0.19
\end{array}
$$

$\begin{array}{llllllllll}0.19 & 0.19 & 0.20 & 0.22 & 0.22 & 0.21 & 0.17 & 0.15 & 0.15 & 0.16\end{array}$
0.17

$$
4.129
$$

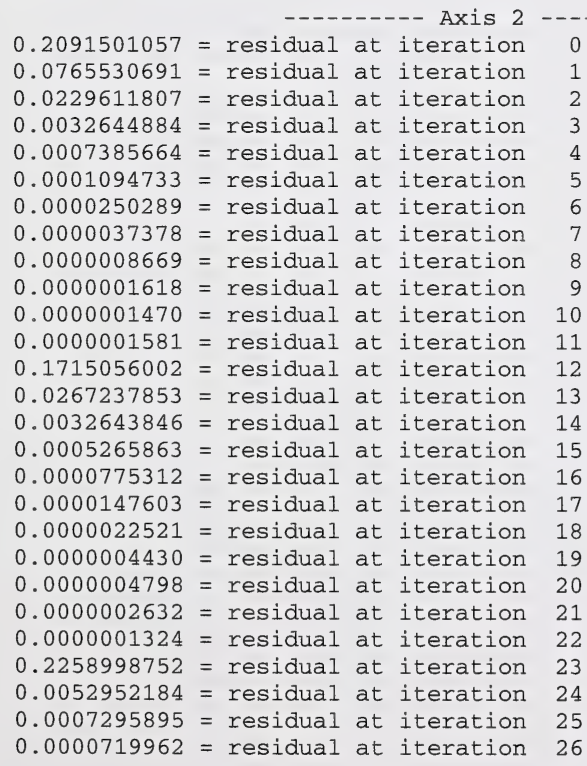


$0.0000153151=$ residual at iteration 27

0.0000024945 = residual at iteration 28

$0.0000005695=$ residual at iteration 29

0.0000002555 = residual at iteration 30

$0.0000001209=$ residual at iteration 31

0.1649405807 = residual at iteration 32

$0.0030498444=$ residual at iteration 33

0.0001900764 = residual at iteration 34

0.0000162592 = residual at iteration 35

$0.0000029595=$ residual at iteration 36

0.0000004602 = residual at iteration 37

$0.0000002008=$ residual at iteration 38

0.1445547491 = residual at iteration 39

0.0134180319 = residual at iteration 40

$0.0013512193=$ residual at iteration 41

$0.0001006165=$ residual at iteration 42

0.0000235098 = residual at iteration 43

$0.0000029193=$ residual at iteration 44

$0.0000007032=$ residual at iteration 45

0.0000002582 = residual at iteration 46

0.1190573350 = residual at iteration 47

$0.0200145710=$ residual at iteration 48

$0.0045830240=$ residual at iteration 49

0.0006001128 = residual at iteration 50

$0.0000569950=$ residual at iteration 60

0.0000140514 = residual at iteration 70

$0.0000016084=$ residual at iteration 80

$0.0000006614=$ residual at iteration 90

0.0000002011 = residual at iteration 100

0.1686325073 = residual at iteration 110

$0.0000001987=$ residual at iteration 120

0.0245861672 = residual at iteration 130

0.0128338402 = residual at iteration 140

$0.0009726097=$ residual at iteration 150

0.1492897719 = residual at iteration 160

0.0230942033 = residual at iteration 170

$0.0347204730=$ residual at iteration 180

0.0006071823 = residual at iteration 190

0.0000031297 = residual at iteration 200

0.0000002212 = residual at iteration 210

$0.0000000478=$ residual at iteration 220

0.6630458236 = eigenvalue

\begin{tabular}{|c|c|c|c|c|c|c|c|c|c|c|c|}
\hline \multicolumn{12}{|c|}{ Length of gradient: } \\
\hline Length of & segments: & 0.27 & 0.28 & 0.29 & 0.29 & 0.28 & 0.27 & 0.25 & 0.23 & 0.17 & 0.08 \\
\hline Length of & segments: & 0.06 & 0.06 & 0.06 & 0.06 & & & & & & \\
\hline Length of & gradient: & & 499 & & & & & & & & \\
\hline Length of & gradient: & & 663 & & & & & & & & \\
\hline Length of & segments: & 0.11 & 0.11 & 0.12 & 0.14 & 0.19 & 0.23 & 0.25 & 0.25 & 0.24 & 0.24 \\
\hline Length of & segments: & 0.24 & 0.24 & 0.25 & 0.25 & 0.25 & 0.24 & 0.24 & 0.23 & 0.22 & 0.16 \\
\hline Length of & segments: & 0.12 & 0.12 & 0.12 & 0.12 & & & & & & \\
\hline Length of & gradient: & & 639 & & & & & & & & \\
\hline
\end{tabular}

$0.1746972799=$ residual at iteration

0.0342275575 = residual at iteration

0.0056336881 = residual at iteration

0.0004523548 = residual at iteration

0.0000723316 = residual at iteration

$0.0000058883=$ residual at iteration

$0.0000009449=$ residual at iteration

$0.0000000834=$ residual at iteration

0.4721443057 = eigenvalue

Length of gradient:

Length of segments:

Length of segments:

Length of gradient:

Length of gradient:
3.666

$\begin{array}{lllllllllll}0.13 & 0.14 & 0.15 & 0.19 & 0.23 & 0.28 & 0.31 & 0.32 & 0.30 & 0.28 \\ 0.24 & 0.22 & 0.20 & 0.18 & 0.15 & 0.11 & 0.08 & 0.08 & 0.08 & \end{array}$

3.973

3.773
0

1

3

4

5

6

7

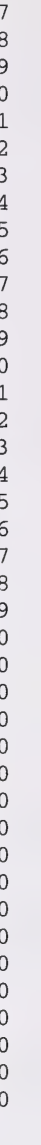


Length of segments:
$\begin{array}{ll}0.17 & 0.17\end{array}$
$0.18 \quad 0.19$
0.20
$\begin{array}{ll}0.21 & 0.21\end{array}$
$\begin{array}{lll}0.22 & 0.23 & 0.24\end{array}$
$0.23 \quad 0.22$
0.20
0.18
0.18
0.18
0.18
0.18
0.19

Length of segments:

3.695

SAND DUNE COMMUNITIES

SPECIES SCORES

\begin{tabular}{|c|c|c|c|c|}
\hline $\mathrm{N}$ & NAME & AX1 & $\mathrm{AX} 2$ & AX3 \\
\hline 1 & POPUDEL1 & -37 & 282 & 387 \\
\hline 2 & POPUTRE2 & -128 & 282 & 356 \\
\hline 3 & POPUDEL2 & 35 & 295 & 443 \\
\hline 4 & SALIAMY3 & 112 & 280 & 387 \\
\hline 5 & ELEACOM4 & -70 & 149 & -9 \\
\hline 6 & SALIAMY 4 & 71 & 330 & 103 \\
\hline 7 & PRUNVIR4 & 428 & -14 & 177 \\
\hline 8 & SALIAMY 5 & 77 & 239 & 101 \\
\hline 9 & ARTECAN5 & -67 & 87 & 395 \\
\hline 10 & RIBEOXY5 & 14 & 262 & 26 \\
\hline 11 & PRUNVIR5 & 424 & -15 & 180 \\
\hline 12 & ELAECOM5 & -27 & 91 & -43 \\
\hline 13 & SALIEXI5 & -24 & 175 & -13 \\
\hline 14 & EUROLAN5 & 206 & 112 & 282 \\
\hline 15 & ROSAWOO5 & 116 & 308 & 38 \\
\hline 16 & SOLIMIS6 & 104 & 197 & 201 \\
\hline 17 & PLANPAT6 & -425 & 49 & 370 \\
\hline 18 & ARTECAM & 228 & 94 & 306 \\
\hline 19 & ERIGCAE6 & -67 & 87 & 395 \\
\hline 20 & POTEPEN6 & -67 & 87 & 395 \\
\hline 21 & ANTEPAR6 & -67 & 87 & 395 \\
\hline 22 & LEPIDEN6 & -46 & 70 & 380 \\
\hline 23 & ACHIMIL6 & -99 & 96 & 353 \\
\hline 24 & CLEOSER6 & 66 & 184 & 391 \\
\hline 25 & CORYMIS6 & -136 & 111 & 338 \\
\hline 26 & SALSKAL 6 & 398 & 1 & 270 \\
\hline 27 & HELISUB6 & 35 & 295 & 443 \\
\hline 28 & EQUIFLU6 & 380 & 3 & 364 \\
\hline 29 & ARTELUD6 & 26 & 137 & 187 \\
\hline 30 & THERRHO6 & 134 & 347 & 210 \\
\hline 31 & TARAOFF6 & 110 & 289 & 406 \\
\hline 32 & TRAGDUB6 & -54 & 230 & 300 \\
\hline 33 & CRYPFEN6 & 281 & 40 & 261 \\
\hline 34 & CHENSUB6 & 211 & 136 & 250 \\
\hline 35 & CHENPRA 6 & 165 & 161 & 329 \\
\hline 36 & DESCSOP6 & -20 & 192 & 378 \\
\hline 37 & SMILSTE6 & -69 & 265 & 388 \\
\hline 38 & ARTEFRI6 & 117 & 127 & 319 \\
\hline 39 & SOLICAN6 & -47 & 249 & 251 \\
\hline 40 & CHENFRE6 & 226 & 197 & 310 \\
\hline 41 & LYGOROS6 & 361 & 37 & 60 \\
\hline 42 & OPUNPOL6 & 191 & 78 & 290 \\
\hline 43 & OENONUT6 & -192 & 233 & 93 \\
\hline 44 & RUMEVEN6 & 405 & 496 & 182 \\
\hline 45 & FRANACA6 & 402 & 490 & 216 \\
\hline 46 & CHRYVIL6 & 197 & 168 & 254 \\
\hline 47 & GLYCLEP6 & 30 & 213 & 189 \\
\hline 48 & ANTIMIC6 & 77 & 239 & 101 \\
\hline 49 & LATUPUL 6 & 15 & 265 & 16 \\
\hline 50 & HEL IANN6 & 46 & 245 & 199 \\
\hline 51 & CERAARV6 & 96 & 89 & 205 \\
\hline 52 & BOUTGRA7 & 12 & 280 & 387 \\
\hline 53 & CYPESCH7 & 175 & 110 & 290 \\
\hline 54 & JUNCBAL 7 & 17 & 249 & 227 \\
\hline 55 & STIPCOM7 & 135 & 96 & 334 \\
\hline 56 & KOELMAC 7 & 112 & 131 & 234 \\
\hline 57 & AGRODAS7 & 235 & 57 & 76 \\
\hline 58 & AGROSMI7 & 370 & 37 & 313 \\
\hline 59 & AGROSIB7 & 60 & 278 & 87 \\
\hline 60 & CARELAN7 & 81 & 292 & 31 \\
\hline 61 & POAPRA7 & -55 & 241 & 291 \\
\hline 62 & ORYZHYM7 & 351 & 350 & 196 \\
\hline
\end{tabular}

RANKED 1

$E I G=0.786$

7 PRUNVIR4

11 PRUNVIR5

44 RUMEVEN6

45 FRANACA6

26 SALSKAL6

28 EQUIFLU6

58 AGROSMI7

41 LYGOROS6

62 ORYZHYM7

50 HELIANN6

63 ELYMCAN7

51 CERAARV6

33 CRYPFEN6

65 CALALON7

64 SPORCRY7

57 AGRODAS7

18 ARTECAM

40 CHENFRE6

49 LATUPUL6

34 CHENSUB6

14 EUROLAN5

46 CHRYVIL6

42 OPUNPOL6

53 CYPESCH7

35 CHENPRA6

55 STIPCOM7

30 THERRHO 6

38 ARTEFRI6

15 ROSAWOO5

4 SALIAMY 3

52 BOUTGRA7

56 KOELMAC7

31 TARAOFF 6

16 SOLIMIS6

60 CARELAN7

8 SALIAMY 5

48 ANTIMIC6

6 SALIAMY 4

24 CLEOSER6

59 AGROSIB7

3 POPUDEL2

27 HELISUB6

47 GLYCLEP6

29 ARTELUD6

54 JUNCBAL7

10 RIBEOXY5

36 DESCSOP6

13 SALIEXI5

12 ELAECOM5

1 POPUDEL1

22 LEPIDEN6

39 SOLICAN6

32 TRAGDUB6

61 POAPRA7

21 ANTEPAR6

19 ERIGCAE6

9 ARTECAN5

20 POTEPEN6

37 SMILSTE6

5 ELEACOM4

23 ACHIMIL6

2 POPUTRE2
1 RANKED 2

$E I G=0.663$

44 RUMEVEN6

45 FRANACA6

63 ELYMCAN7

62 ORYZHYM7

30 THERRHO6

6 SALIAMY 4

15 ROSAWOO5

27 HELISUB6

3 POPUDEL2

60 CARELAN7

64 SPORCRY7

31 TARAOFF6

1 POPUDEL1

2 POPUTRE2

4 SALIAMY3

52 BOUTGRA7

59 AGROSIB7

37 SMILSTE6

49 LATUPUL6

10 RIBEOXY 5

39 SOLICAN6

54 JUNCBAL7

50 HELIANN6

61 POAPRA 7

8 SALIAMY 5

48 ANTIMIC6

43 OENONUT6

32 TRAGDUB6

47 GLYCLEP6

16 SOLIMIS6

40 CHENFRE6

36 DESCSOP6

24 CLEOSER6

13 SALIEXI5

46 CHRYVIL6

35 CHENPRA 6

5 ELEACOM4

29 ARTELUD6

34 CHENSUB6

56 KOELMAC 7

38 ARTEFRI6

14 EUROLAN5

25 CORYMIS 6

53 CYPESCH7

23 ACHIMIL6

55 STIPCOM7

18 ARTECAM

12 ELAECOM 5

51 CERAARV6

9 ARTECAN5

19 ERIGCAE6

20 POTEPEN6

21 ANTEPAR6

42 OPUNPOL 6

65 CALALON7

22 LEPIDEN6

57 AGRODAS7

17 PLANPAT6

33 CRYPFEN6

58 AGROSMI7

41 LYGOROS6

28 EQUIFLU6

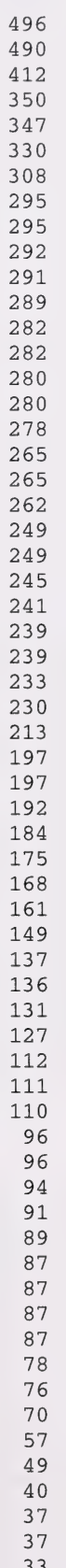

496

12

47

308

95

92

291

82

280

265

65

249

41

239

233

197

84

168

61

137

136

27

112

110 
$\begin{array}{llrrr}63 & \text { ELYMCAN7 } & 305 & 412 & 128 \\ 64 & \text { SPORCRY7 } & 236 & 291 & 73 \\ 65 & \text { CALALON7 } & 246 & 76 & 93\end{array}$
25 CORYMIS6 -136

43 OENONUT6 -192

17 PLANPAT6 -425
26 SALSKAL6

7 PRUNVIR4 -14

11 PRUNVIR5 -15

SAND DUNE COMMUNITIES

SAMPLE SCORES - WHICH ARE WEIGHTED MEAN SPECIES SCORES

\begin{tabular}{|c|c|c|c|c|c|c|c|c|c|c|}
\hline $\mathrm{N}$ & NAME & AX1 & $\mathrm{AX} 2$ & $\mathrm{AX} 3$ & \multicolumn{3}{|c|}{$\begin{array}{r}\text { RANKED } 1 \\
\text { EIG }=0.786\end{array}$} & \multicolumn{3}{|c|}{$\begin{array}{r}\text { RANKED } 2 \\
\text { EIG }=0.663\end{array}$} \\
\hline 1 & 1 & 257 & 248 & 141 & & 10 & 412 & 30 & & 463 \\
\hline 2 & 2 & 19 & 225 & 175 & 14 & 14 & 412 & 6 & 6 & 461 \\
\hline 3 & 3 & 239 & 252 & 87 & 6 & 6 & 390 & 29 & 29 & 314 \\
\hline 4 & 4 & 206 & 219 & 104 & 13 & 13 & 371 & 31 & 31 & 310 \\
\hline 5 & 5 & 140 & 293 & 55 & 30 & 30 & 367 & 21 & 21 & 305 \\
\hline 6 & 6 & 390 & 461 & 183 & 34 & 34 & 362 & 35 & 35 & 303 \\
\hline 7 & 7 & 42 & 215 & 195 & 21 & 21 & 324 & 28 & 28 & 303 \\
\hline 8 & 8 & 235 & 140 & 252 & 37 & 37 & 307 & 26 & 26 & 300 \\
\hline 9 & 9 & 0 & 254 & 278 & 35 & 35 & 282 & 37 & 37 & 297 \\
\hline 10 & 10 & 412 & 15 & 178 & 29 & 29 & 263 & 5 & 5 & 293 \\
\hline 11 & 11 & 33 & 168 & 132 & 1 & 1 & 257 & 27 & 27 & 279 \\
\hline 12 & 12 & 46 & 257 & 369 & 25 & 25 & 250 & 25 & 25 & 265 \\
\hline 13 & 13 & 371 & 72 & 170 & 3 & 3 & 239 & 12 & 12 & 257 \\
\hline 14 & 14 & 412 & 0 & 177 & 8 & 8 & 235 & 9 & 9 & 254 \\
\hline 15 & 15 & 230 & 196 & 186 & 15 & 15 & 230 & 17 & 17 & 253 \\
\hline 16 & 16 & 119 & 244 & 101 & 31 & 31 & 214 & 3 & 3 & 252 \\
\hline 17 & 17 & 13 & 253 & 307 & 4 & 4 & 206 & 1 & 1 & 248 \\
\hline 18 & 18 & 9 & 119 & 0 & 23 & 23 & 182 & 16 & 16 & 244 \\
\hline 19 & 19 & 46 & 176 & 218 & 33 & 33 & 176 & 2 & 2 & 225 \\
\hline 20 & 20 & 92 & 156 & 261 & 38 & 38 & 169 & 4 & 4 & 219 \\
\hline 21 & 21 & 324 & 305 & 185 & 40 & 40 & 163 & 7 & 7 & 215 \\
\hline 22 & 22 & 136 & 128 & 291 & 26 & 26 & 150 & 15 & 15 & 196 \\
\hline 23 & 23 & 182 & 146 & 105 & 5 & 5 & 140 & 19 & 19 & 176 \\
\hline 24 & 24 & 110 & 116 & 316 & 39 & 39 & 140 & 11 & 11 & 168 \\
\hline 25 & 25 & 250 & 265 & 197 & 22 & 22 & 136 & 36 & 36 & 157 \\
\hline 26 & 26 & 150 & 300 & 60 & 28 & 28 & 130 & 20 & 20 & 156 \\
\hline 27 & 27 & 99 & 279 & 133 & 16 & 16 & 119 & 23 & 23 & 146 \\
\hline 28 & 28 & 130 & 303 & 119 & 36 & 36 & 115 & 8 & 8 & 140 \\
\hline 29 & 29 & 263 & 314 & 125 & 24 & 24 & 110 & 22 & 22 & 128 \\
\hline 30 & 30 & 367 & 463 & 169 & 32 & 32 & 99 & 18 & 18 & 119 \\
\hline 31 & 31 & 214 & 310 & 135 & 27 & 27 & 99 & 24 & 24 & 116 \\
\hline 32 & 32 & 99 & 95 & 63 & 20 & 20 & 92 & 38 & 38 & 114 \\
\hline 33 & 33 & 176 & 102 & 259 & 19 & 19 & 46 & 40 & 40 & 107 \\
\hline 34 & 34 & 362 & 21 & 181 & 12 & 12 & 46 & 33 & 33 & 102 \\
\hline 35 & 35 & 282 & 303 & 131 & 7 & 7 & 42 & 39 & 39 & 101 \\
\hline 36 & 36 & 115 & 157 & 181 & 11 & 11 & 33 & 32 & 32 & 95 \\
\hline 37 & 37 & 307 & 297 & 156 & 2 & 2 & 19 & 13 & 13 & 72 \\
\hline 38 & 38 & 169 & 114 & 276 & 17 & 17 & 13 & 34 & 34 & 21 \\
\hline 39 & 39 & 140 & 101 & 284 & 18 & 18 & 9 & 10 & 10 & 15 \\
\hline 40 & 40 & 163 & 107 & 273 & 9 & 9 & 0 & 14 & 14 & 0 \\
\hline
\end{tabular}

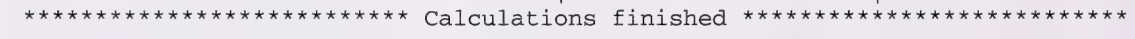




Appendix 3. Species Code Descriptions 



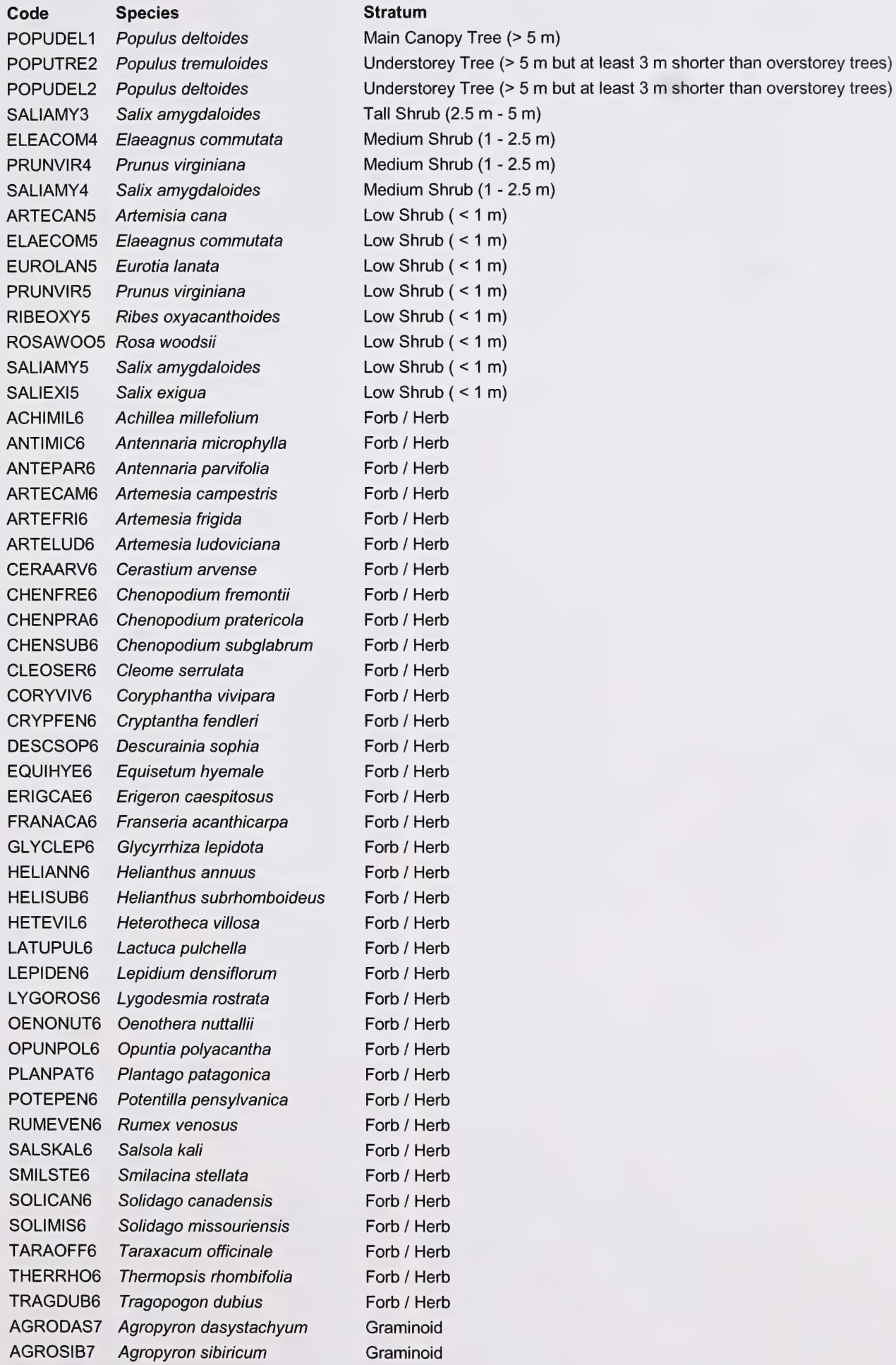

\section{Code Species}

POPUDEL1 Populus deltoides

POPUTRE2 Populus tremuloides

POPUDEL2 Populus deltoides

SALIAMY3 Salix amygdaloides

ELEACOM4 Elaeagnus commutata

PRUNVIR4 Prunus virginiana

SALIAMY4 Salix amygdaloides

ARTECAN5 Artemisia cana

ELAECOM5 Elaeagnus commutata

EUROLAN5 Eurotia lanata

PRUNVIR5 Prunus virginiana

RIBEOXY5 Ribes oxyacanthoides

ROSAWOO5 Rosa woodsii

SALIAMY5 Salix amygdaloides

SALIEXI5 Salix exigua

ACHIMIL6 Achillea millefolium

ANTIMIC6 Antennaria microphylla

ANTEPAR6 Antennaria parvifolia

ARTECAM6 Artemesia campestris

ARTEFRI6 Artemesia frigida

ARTELUD6 Artemesia ludoviciana

CERAARV6 Cerastium arvense

CHENFRE6 Chenopodium fremontii

CHENPRA6 Chenopodium pratericola

CHENSUB6 Chenopodium subglabrum

CLEOSER6 Cleome serrulata

CORYVIV6 Coryphantha vivipara

CRYPFEN6 Cryptantha fendleri

DESCSOP6 Descurainia sophia

EQUIHYE6 Equisetum hyemale

ERIGCAE6 Erigeron caespitosus

FRANACA6 Franseria acanthicarpa

GLYCLEP6 Glycyrrhiza lepidota

HELIANN6 Helianthus annuus

HELISUB6 Helianthus subrhomboideus

HETEVIL6 Heterotheca villosa

LATUPUL6 Lactuca pulchella

LEPIDEN6 Lepidium densiflorum

LYGOROS6 Lygodesmia rostrata

OENONUT6 Oenothera nuttallii

OPUNPOL6 Opuntia polyacantha

PLANPAT6 Plantago patagonica

POTEPEN6 Potentilla pensylvanica

RUMEVEN6 Rumex venosus

SALSKAL6 Salsola kali

SMILSTE6 Smilacina stellata

SOLICAN6 Solidago canadensis

SOLIMIS6 Solidago missouriensis

TARAOFF6 Taraxacum officinale

THERRHO6 Thermopsis rhombifolia

TRAGDUB6 Tragopogon dubius

AGRODAS7 Agropyron dasystachyum

AGROSIB7 Agropyron sibiricum

Stratum

Main Canopy Tree (> $5 \mathrm{~m}$ )

Understorey Tree (> $5 \mathrm{~m}$ but at least $3 \mathrm{~m}$ shorter than overstorey trees)

Understorey Tree (> $5 \mathrm{~m}$ but at least $3 \mathrm{~m}$ shorter than overstorey trees)

Tall Shrub (2.5 m - $5 \mathrm{~m})$

Medium Shrub (1 $-2.5 \mathrm{~m})$

Medium Shrub (1 - $2.5 \mathrm{~m})$

Medium Shrub (1 - $2.5 \mathrm{~m})$

Low Shrub $(<1 \mathrm{~m})$

Low Shrub $(<1 \mathrm{~m})$

Low Shrub $(<1 \mathrm{~m})$

Low Shrub $(<1 \mathrm{~m})$

Low Shrub $(<1 \mathrm{~m})$

Low Shrub $(<1 \mathrm{~m})$

Low Shrub $(<1 \mathrm{~m})$

Low Shrub $(<1 \mathrm{~m})$

Forb / Herb

Forb / Herb

Forb / Herb

Forb / Herb

Forb / Herb

Forb / Herb

Forb / Herb

Forb / Herb

Forb / Herb

Forb / Herb

Forb / Herb

Forb / Herb

Forb / Herb

Forb / Herb

Forb / Herb

Forb / Herb

Forb / Herb

Forb / Herb

Forb / Herb

Forb / Herb

Forb / Herb

Forb / Herb

Forb / Herb

Forb / Herb

Forb / Herb

Forb / Herb

Forb / Herb

Forb / Herb

Forb / Herb

Forb / Herb

Forb / Herb

Forb / Herb

Forb / Herb

Forb / Herb

Forb / Herb

Forb / Herb

Graminoid

Graminoid 


$\begin{array}{lll}\text { Code } & \text { Species } & \text { Stratum } \\ \text { AGROSMI7 } & \text { Agropyron smithii } & \text { Graminoid } \\ \text { BOUTGRA7 } & \text { Bouteloua gracilis } & \text { Graminoid } \\ \text { CALALON7 } & \text { Calamovilfa longifolia } & \text { Graminoid } \\ \text { CARELAN7 } & \text { Carex lanuginosa } & \text { Graminoid } \\ \text { CYPESCH7 } & \text { Cyperus schweinitzii } & \text { Graminoid } \\ \text { ELYMCAN7 } & \text { Elymus canadensis } & \text { Graminoid } \\ \text { JUNCBAL7 } & \text { Juncus balticus } & \text { Graminoid } \\ \text { KOELMAC7 } & \text { Koeleria macrantha } & \text { Graminoid } \\ \text { ORYZHYM7 } & \text { Oryzopsis hymenoides } & \text { Graminoid } \\ \text { POAPRA7 } & \text { Poa pratensis } & \text { Graminoid } \\ \text { SPORCRY7 } & \text { Sporobolus cryptandrus } & \text { Graminoid } \\ \text { STIPCOM7 } & \text { Stipa comata } & \text { Graminoid }\end{array}$




Appendix 4. Correlation Table with Literature From Within Alberta 


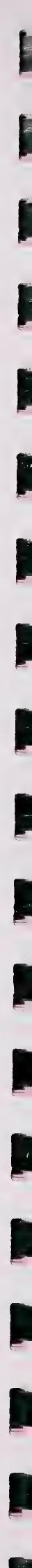




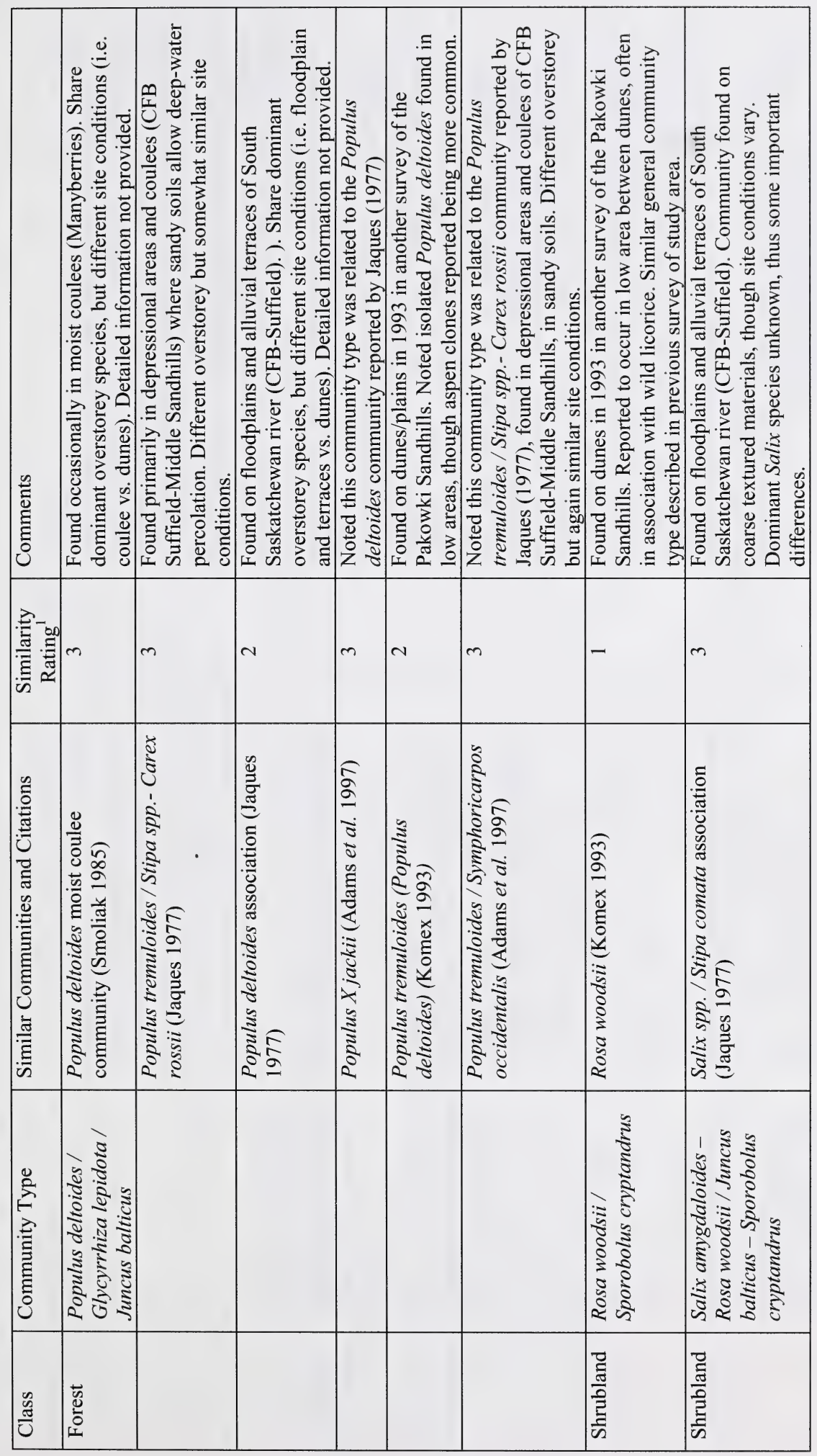

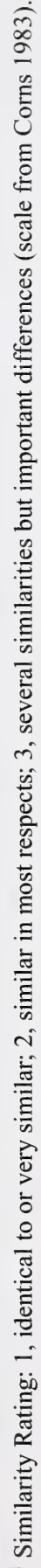




\begin{tabular}{|c|c|c|c|c|c|c|}
\hline 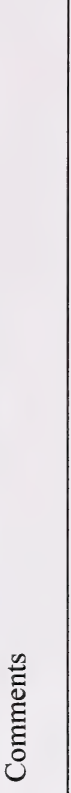 & 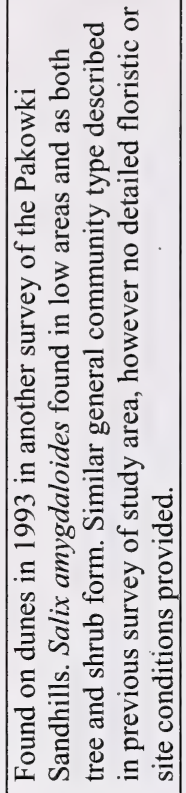 & 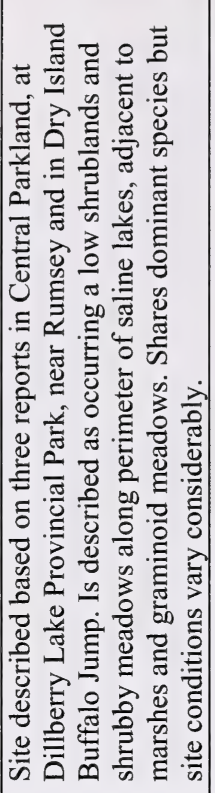 & 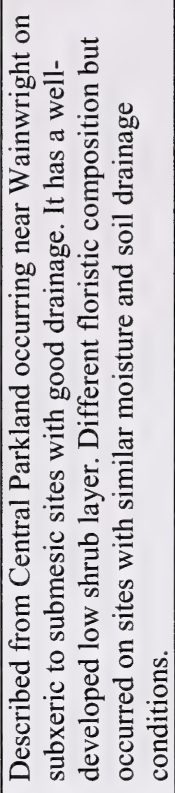 & 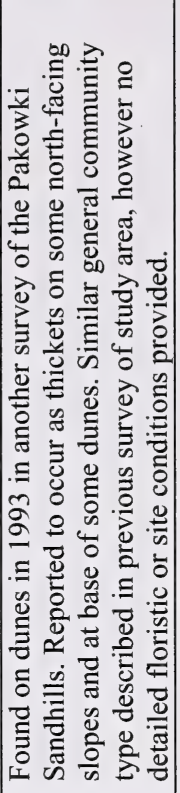 & 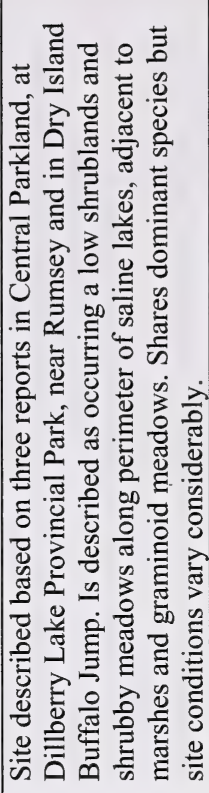 & 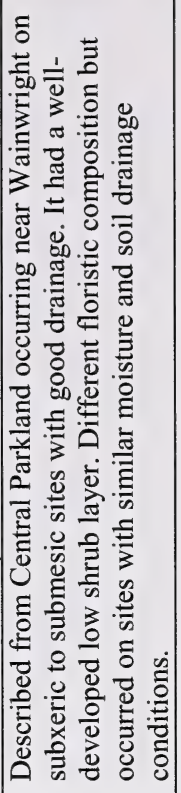 \\
\hline 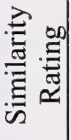 & - & $m$ & $m$ & - & $m$ & $m$ \\
\hline 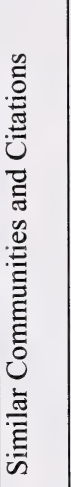 & 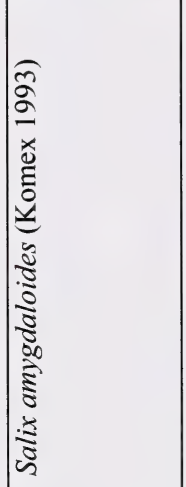 & 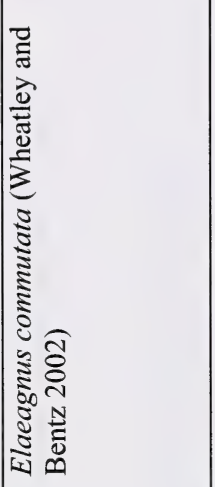 & 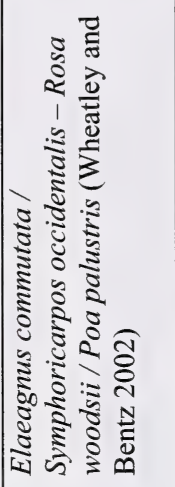 & 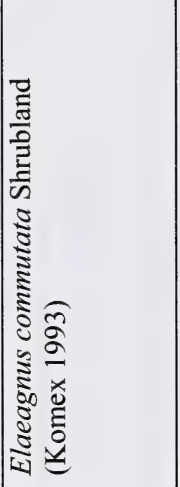 & 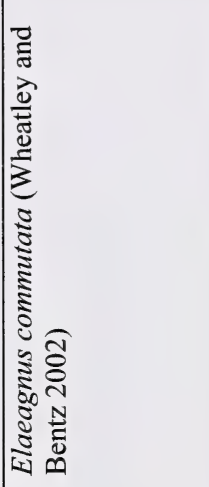 & 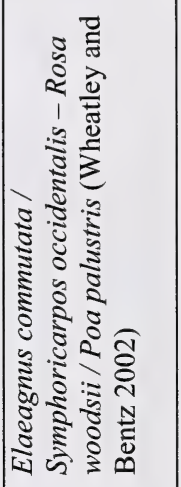 \\
\hline 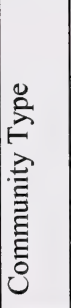 & 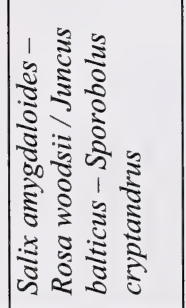 & 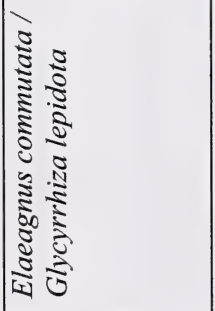 & & & 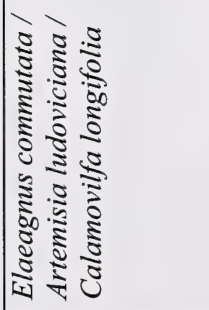 & \\
\hline $\begin{array}{l}\text { Uै } \\
\frac{\tilde{\sigma}}{U}\end{array}$ & 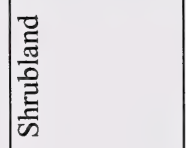 & 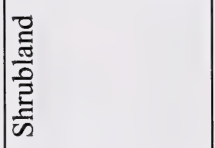 & & & 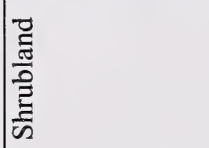 & \\
\hline
\end{tabular}




\begin{tabular}{|c|c|c|c|c|c|c|c|c|c|}
\hline $\begin{array}{l}\text { है } \\
\text { है } \\
\text { हूँ }\end{array}$ & 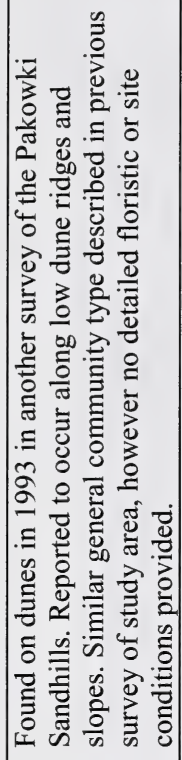 & 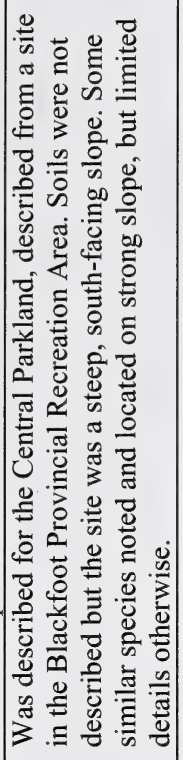 & 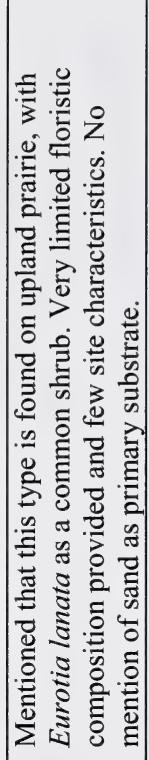 & & 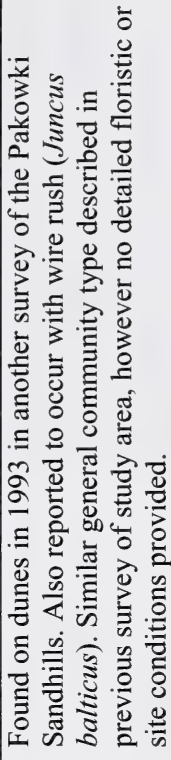 & 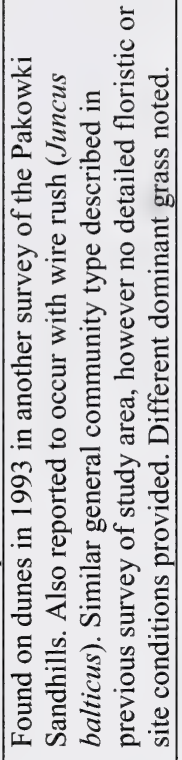 & & 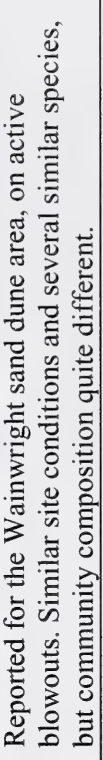 & 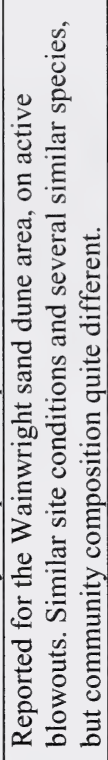 \\
\hline 苞 & - & $m$ & $m$ & & - & $\sim$ & . & $m$ & $m$ \\
\hline 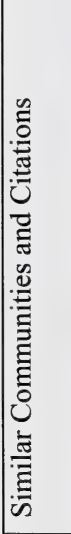 & 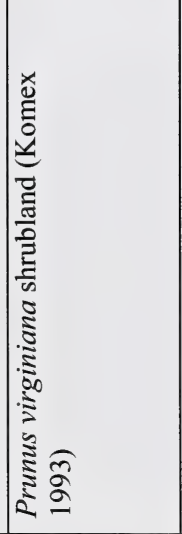 & 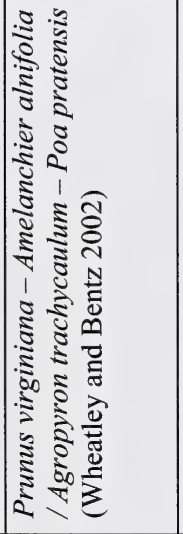 & 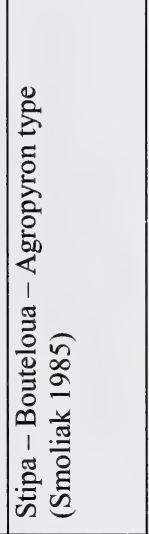 & 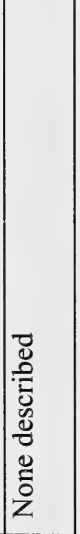 & 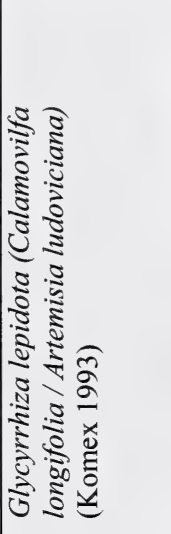 & 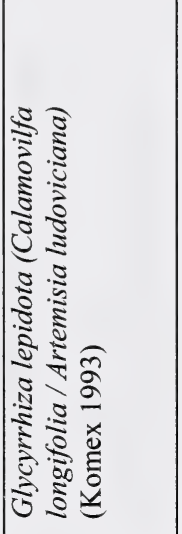 & 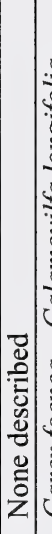 & 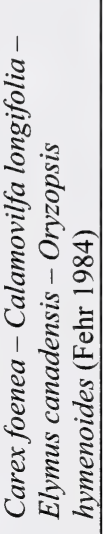 & 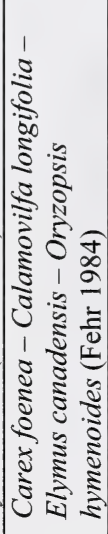 \\
\hline 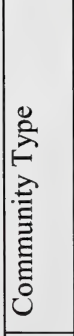 & 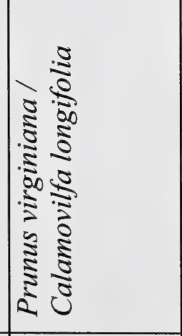 & & 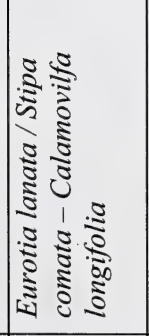 & 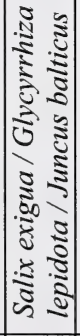 & 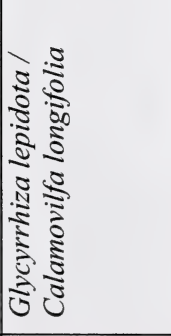 & 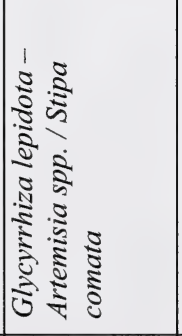 & 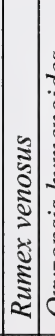 & 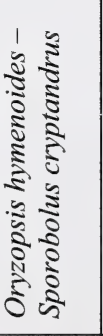 & 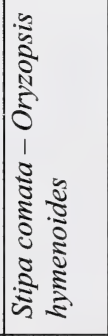 \\
\hline $\begin{array}{l}\frac{w}{\tilde{U}} \\
\frac{\tilde{U}}{2}\end{array}$ & 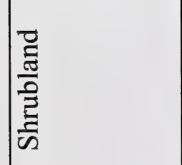 & & 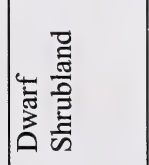 & 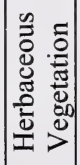 & & 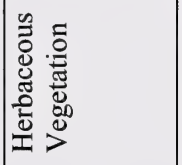 & & & \\
\hline
\end{tabular}




\begin{tabular}{|c|c|c|c|c|c|c|}
\hline 节 & 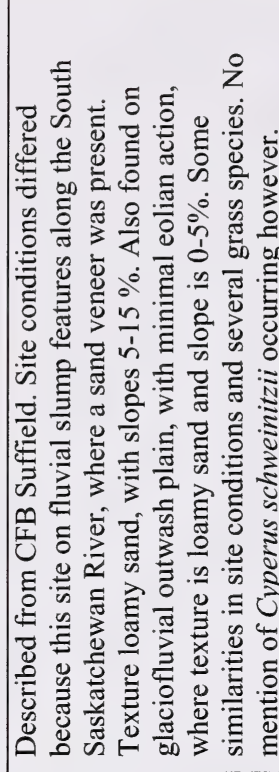 & 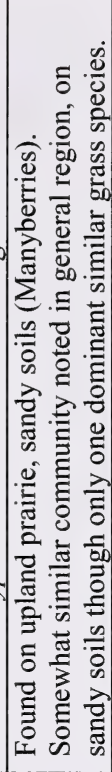 & 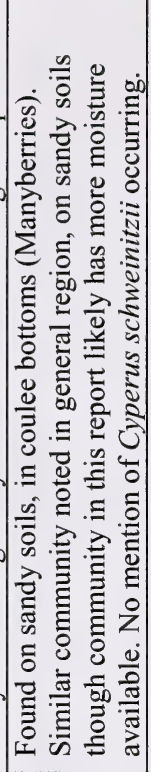 & 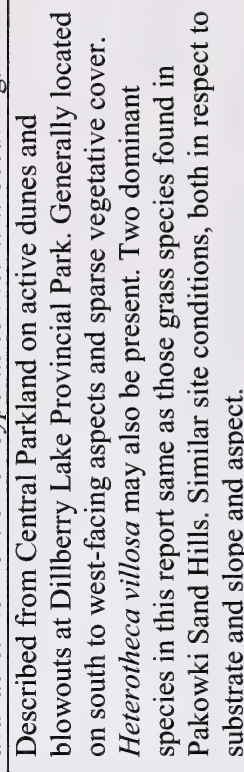 & 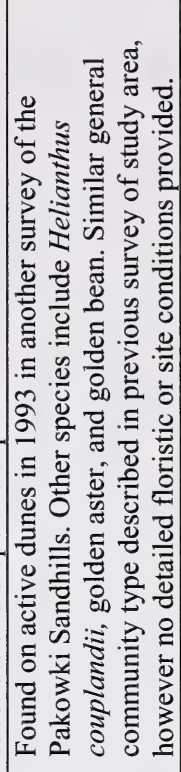 & 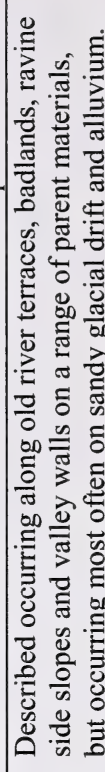 \\
\hline 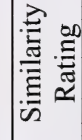 & N & $N$ & $\mathrm{~N}$ & $N$ & - & $N$ \\
\hline 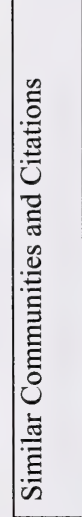 & 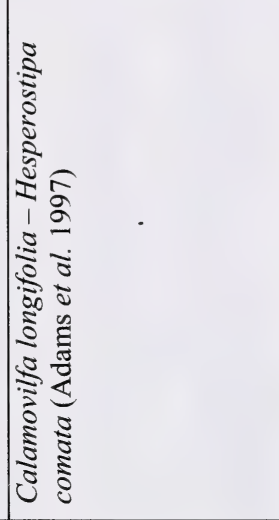 & 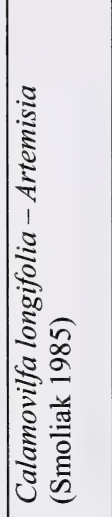 & 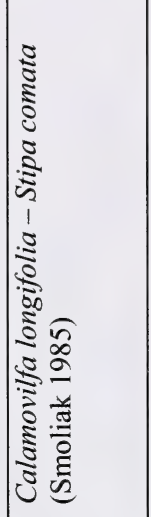 & 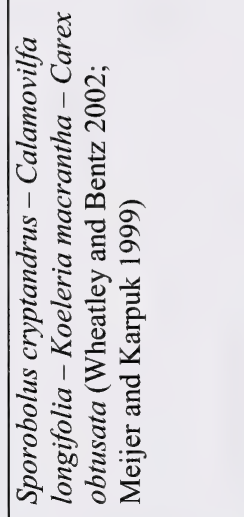 & 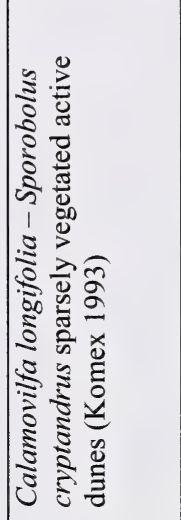 & 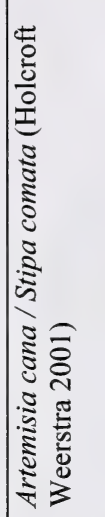 \\
\hline 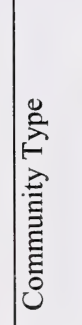 & 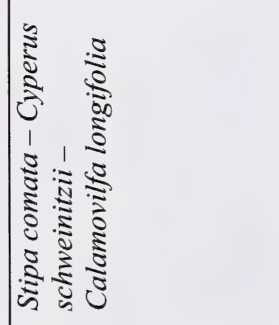 & & & 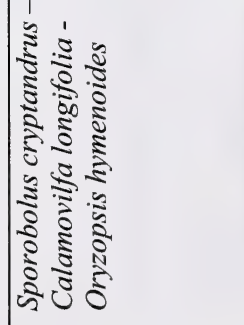 & & 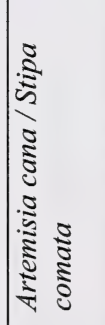 \\
\hline 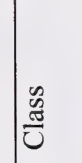 & 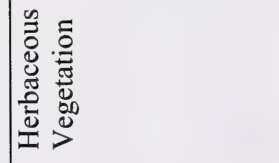 & & & & & 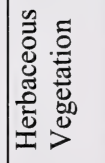 \\
\hline
\end{tabular}




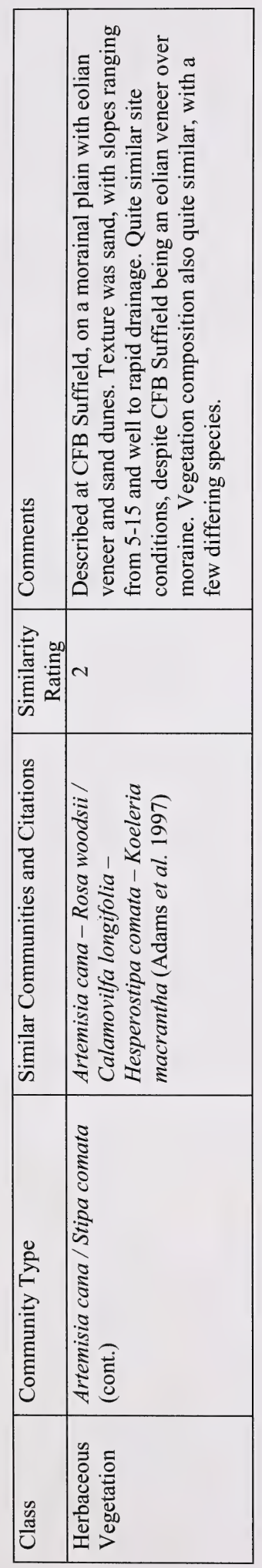





Appendix 5. Correlation Table with Literature From Outside Alberta 


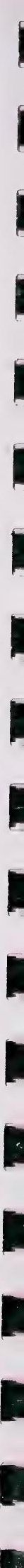




\begin{tabular}{|c|c|c|c|c|}
\hline & 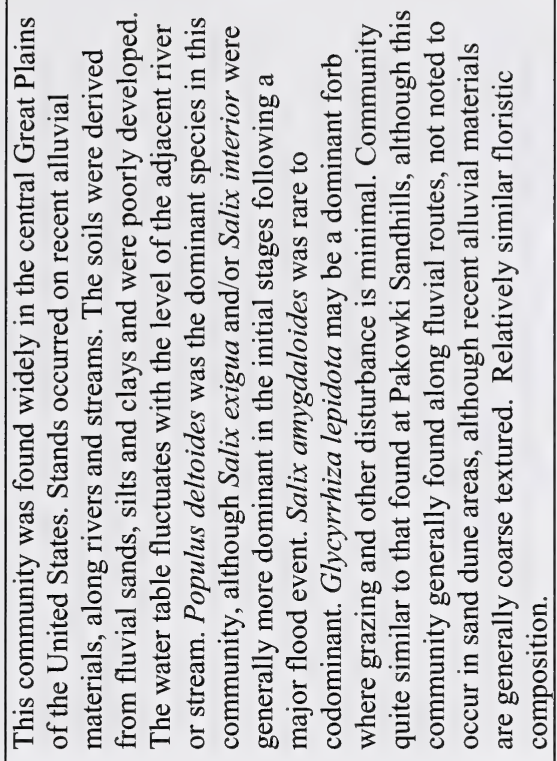 & 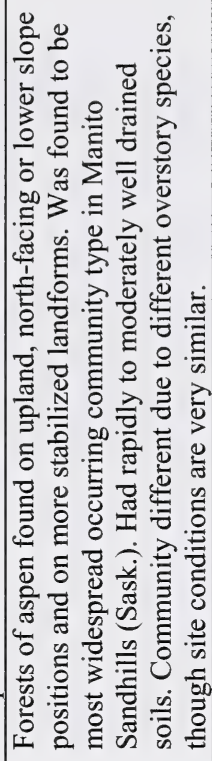 & 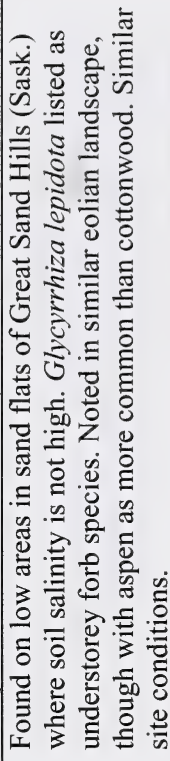 & 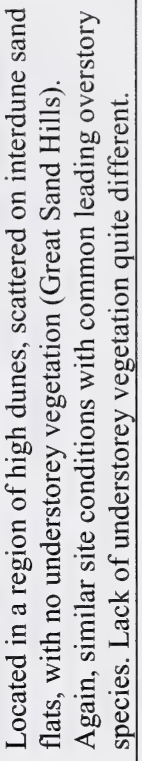 \\
\hline 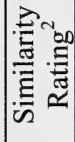 & & $m$ & $N$ & $\sim$ \\
\hline 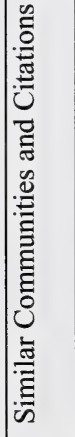 & 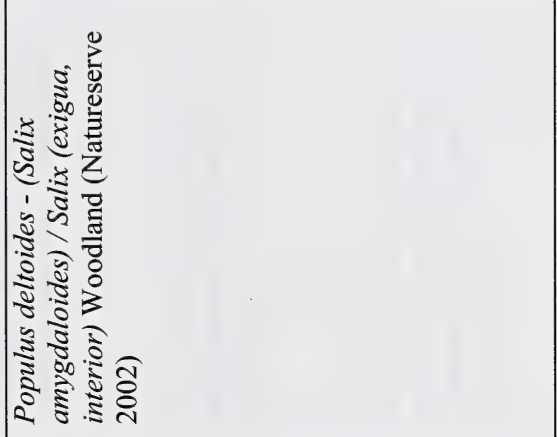 & 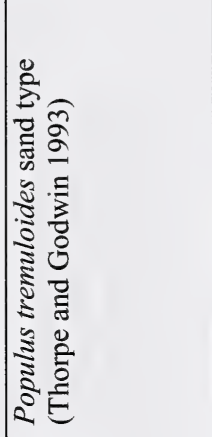 & 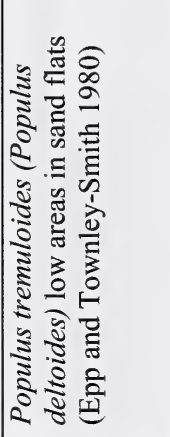 & 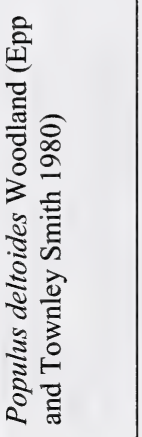 \\
\hline 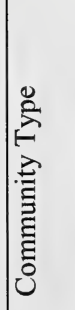 & 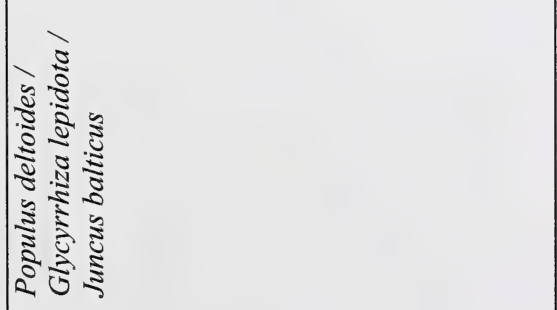 & & & \\
\hline U & 递 & & & \\
\hline
\end{tabular}




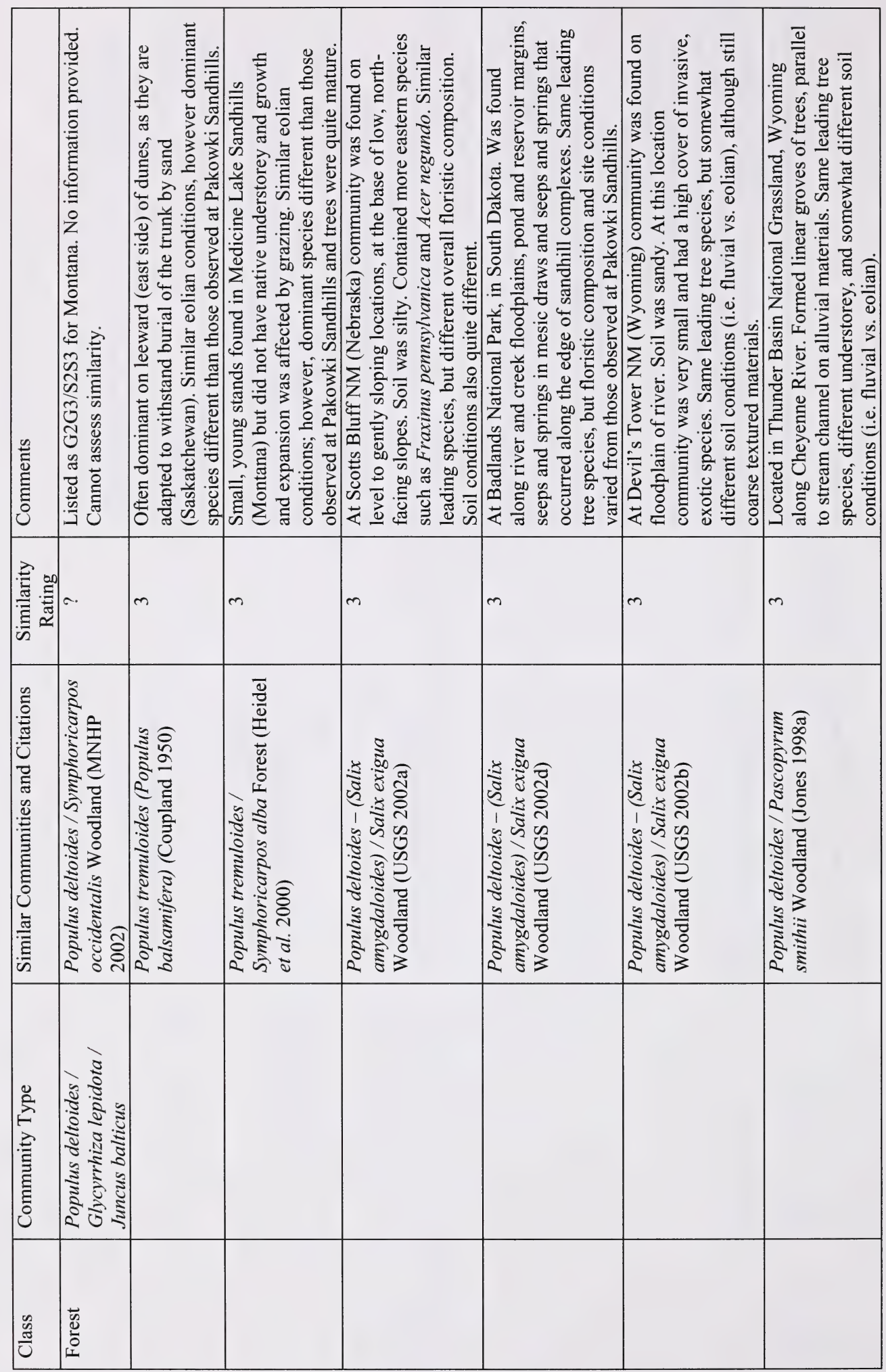




\begin{tabular}{|c|c|c|c|c|}
\hline 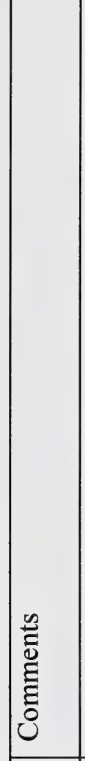 & 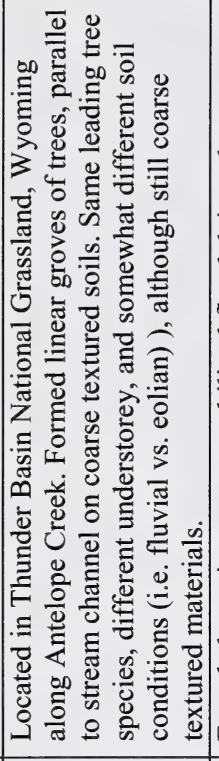 & 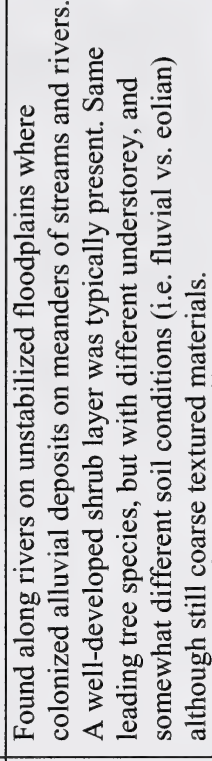 & 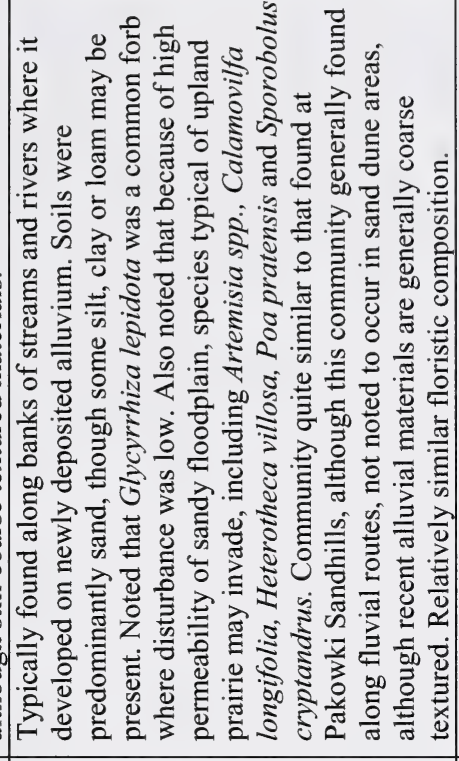 & 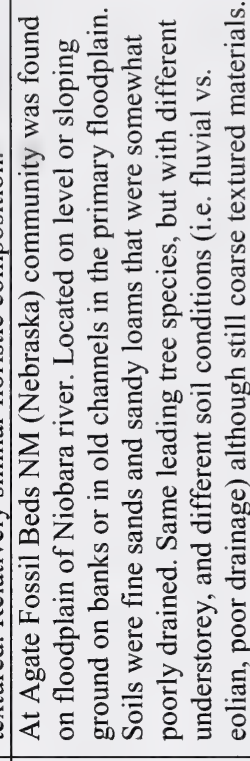 \\
\hline 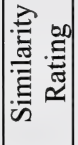 & & $m$ & $\sim$ & $m$ \\
\hline 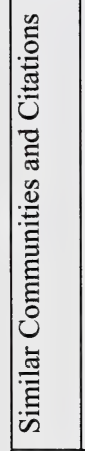 & 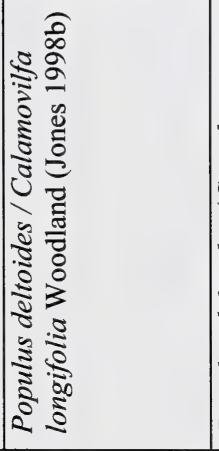 & 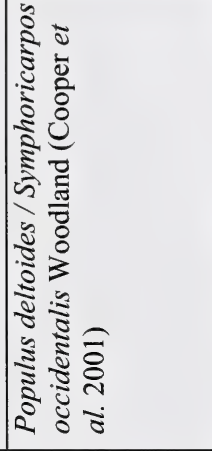 & 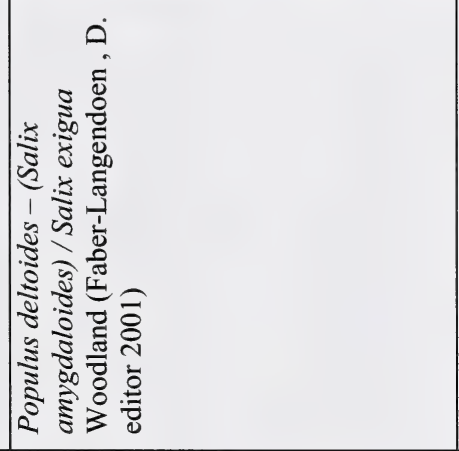 & 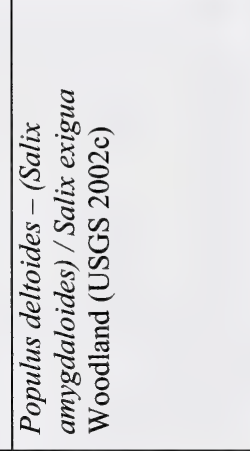 \\
\hline 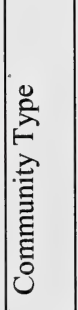 & 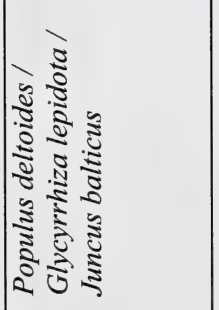 & & & \\
\hline 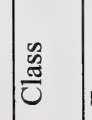 & ర్ & & & \\
\hline
\end{tabular}




\begin{tabular}{|c|c|c|c|c|c|c|}
\hline 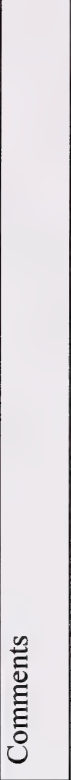 & 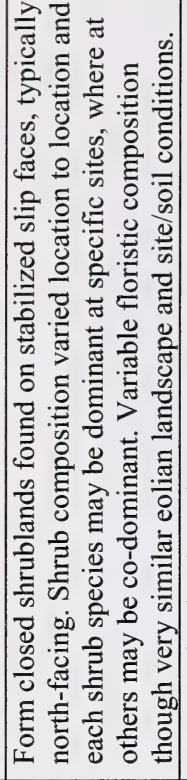 & 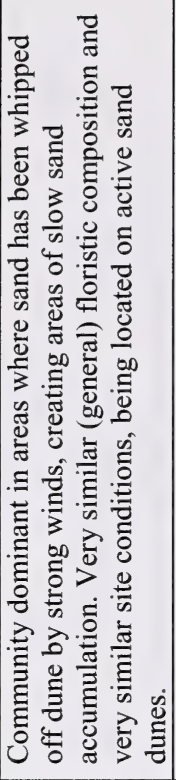 & 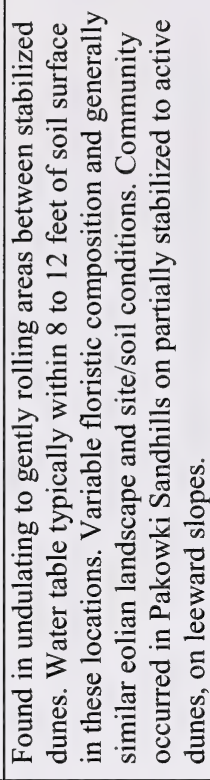 & 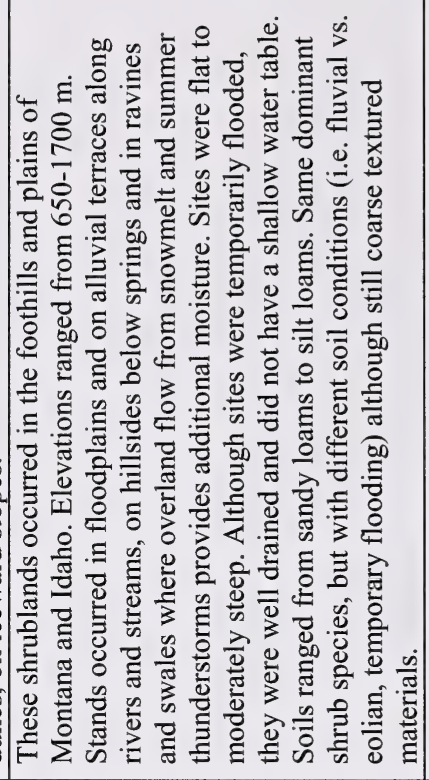 & 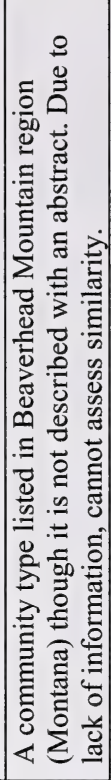 & 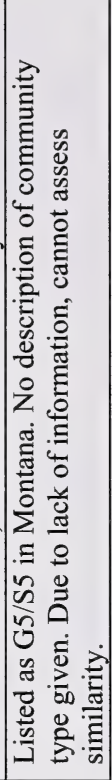 \\
\hline 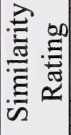 & N & - & $\sim$ & $m$ & r. & ๙. \\
\hline 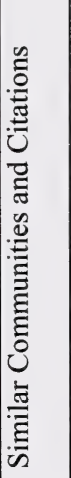 & 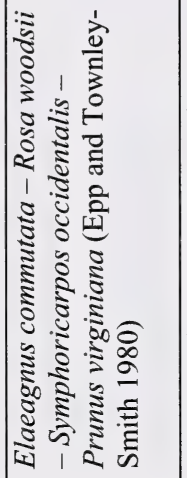 & 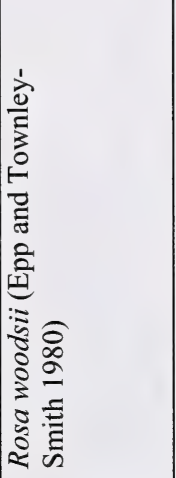 & 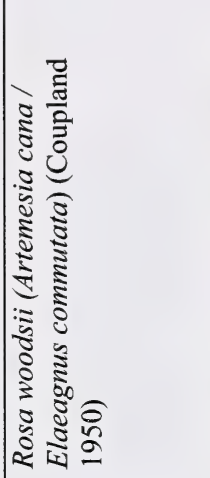 & 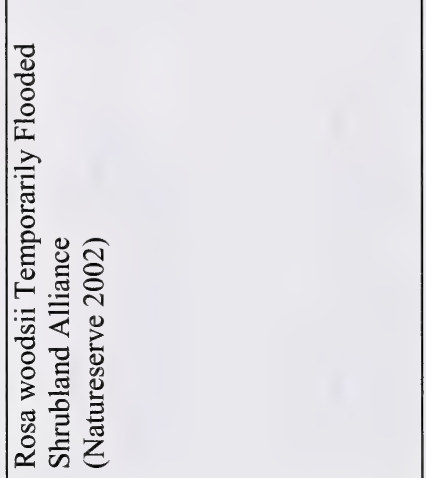 & 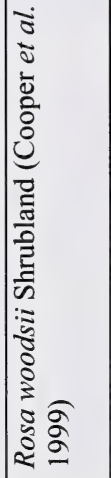 & 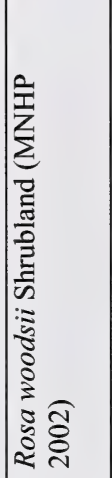 \\
\hline 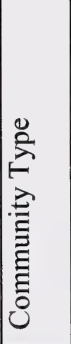 & 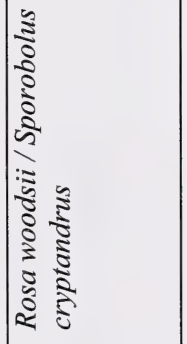 & & & & & \\
\hline & 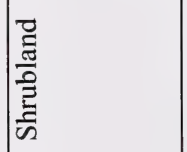 & & & & & \\
\hline
\end{tabular}




\begin{tabular}{|c|c|c|c|c|c|}
\hline 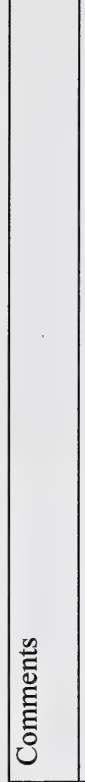 & 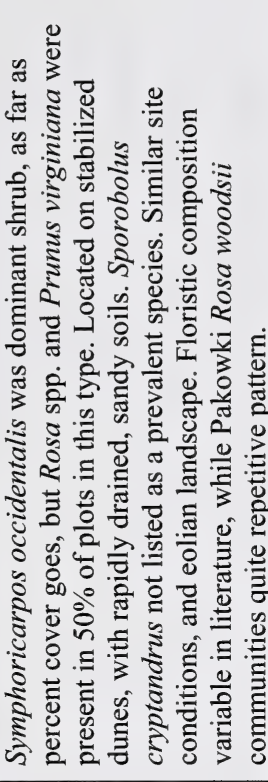 & 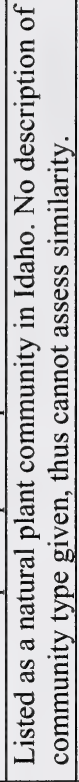 & 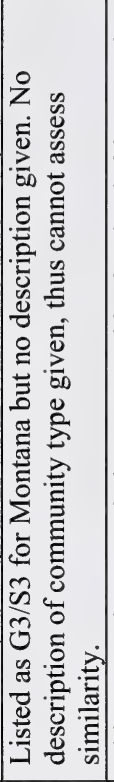 & 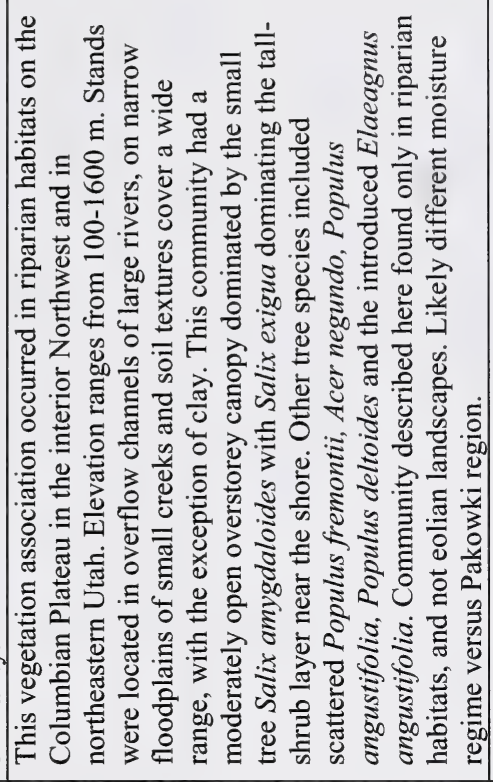 & 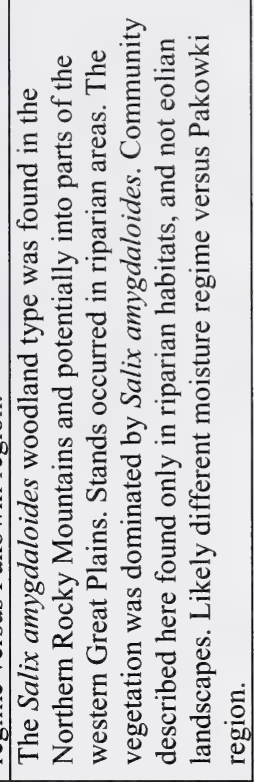 \\
\hline 跣 & $m$ & a. & $\sigma$. & $m$ & $m$ \\
\hline 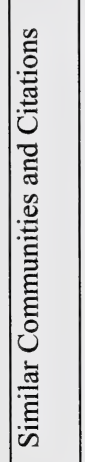 & 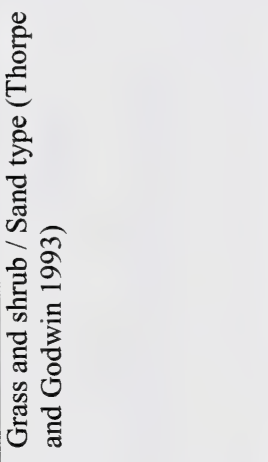 & 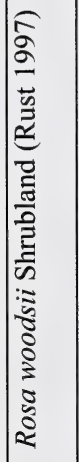 & 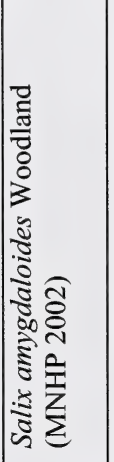 & 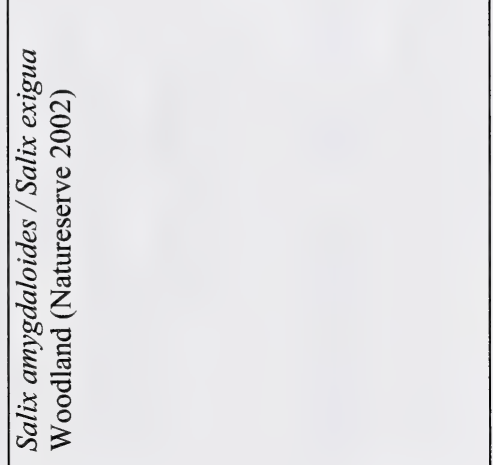 & 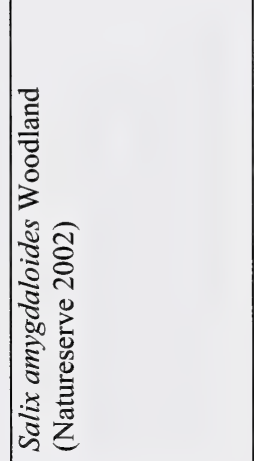 \\
\hline 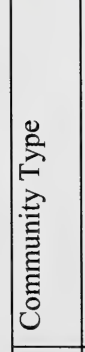 & 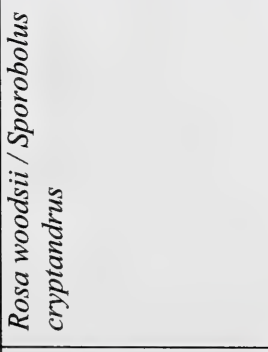 & & 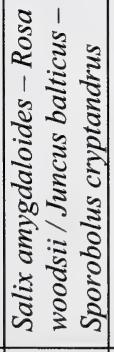 & & \\
\hline 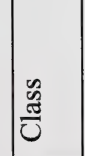 & 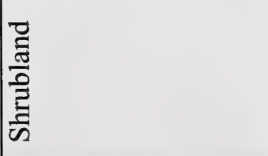 & & 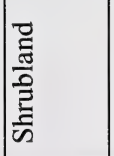 & & \\
\hline
\end{tabular}




\begin{tabular}{|c|c|c|c|c|c|c|}
\hline 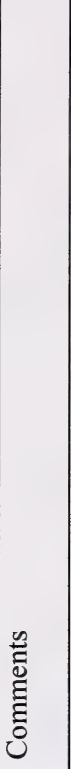 & 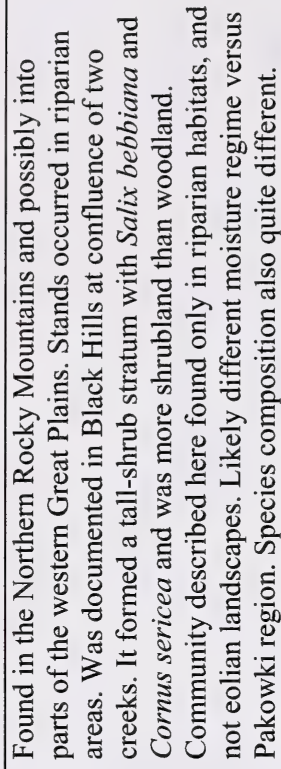 & 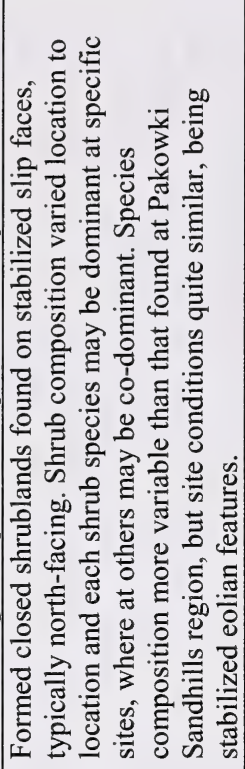 & 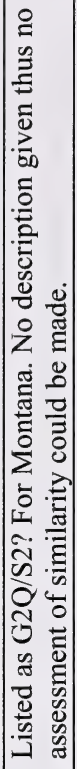 & 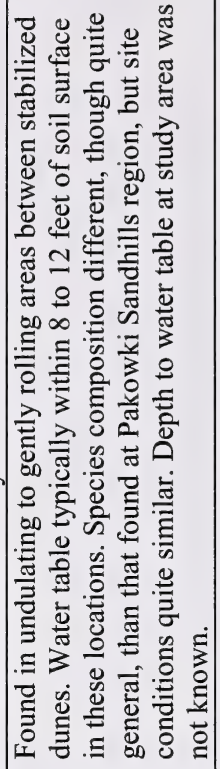 & 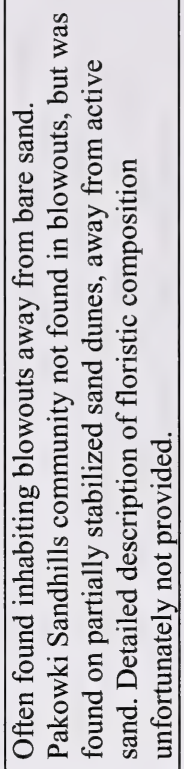 & 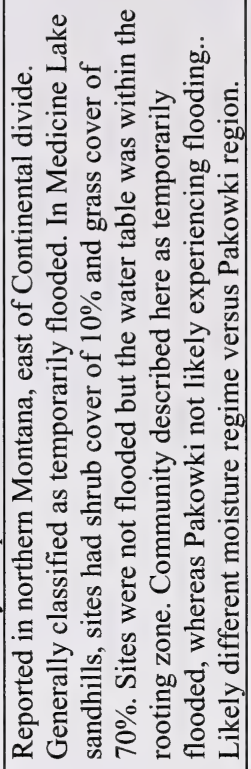 \\
\hline 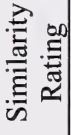 & m & N & r. & $\sim$ & $\sim$ & $\sim$ \\
\hline 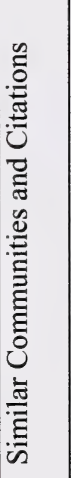 & 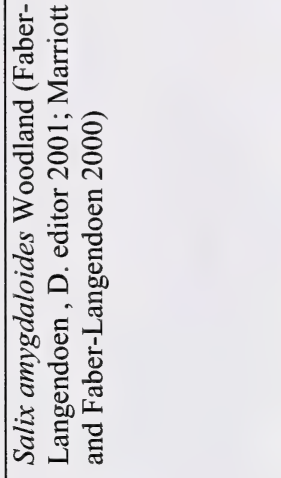 & 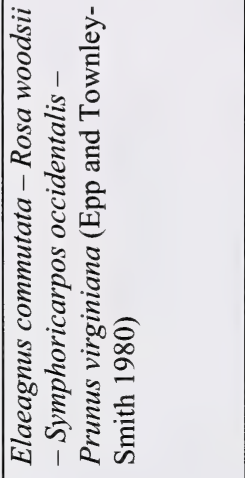 & 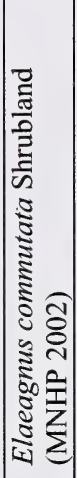 & 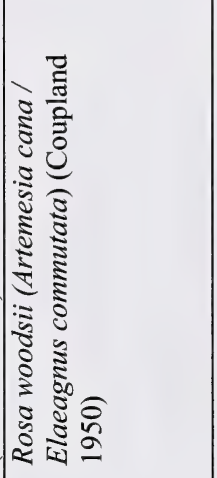 & 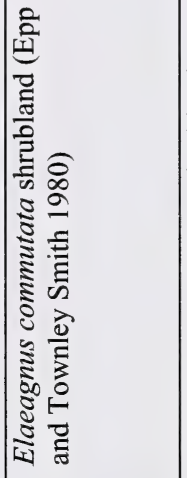 & 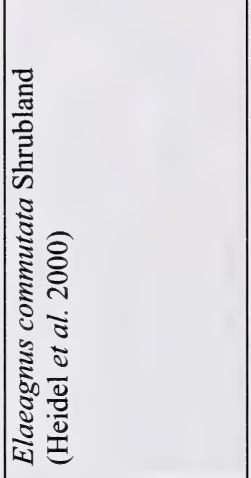 \\
\hline 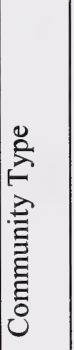 & 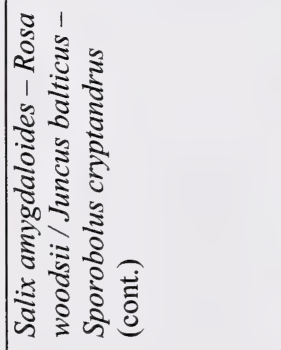 & 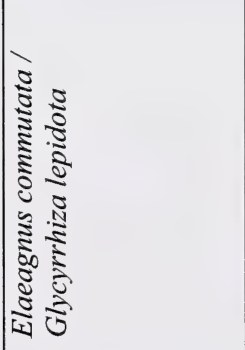 & & & & \\
\hline $\begin{array}{l}\text { के } \\
\text { Uू }\end{array}$ & 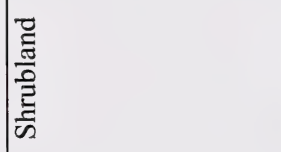 & 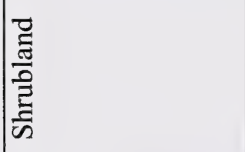 & & & & \\
\hline
\end{tabular}




\begin{tabular}{|c|c|c|c|c|}
\hline ن & 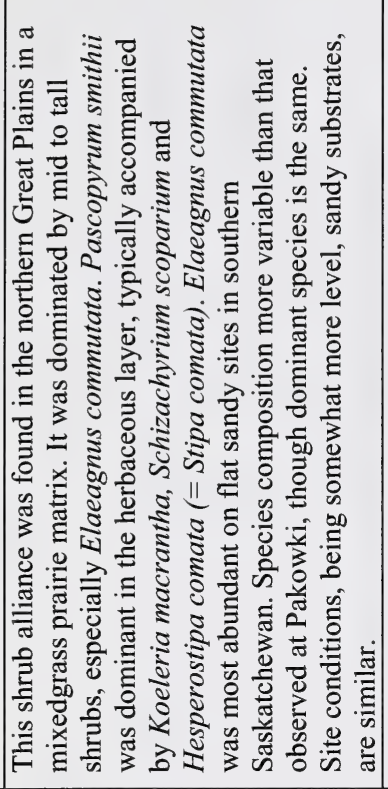 & 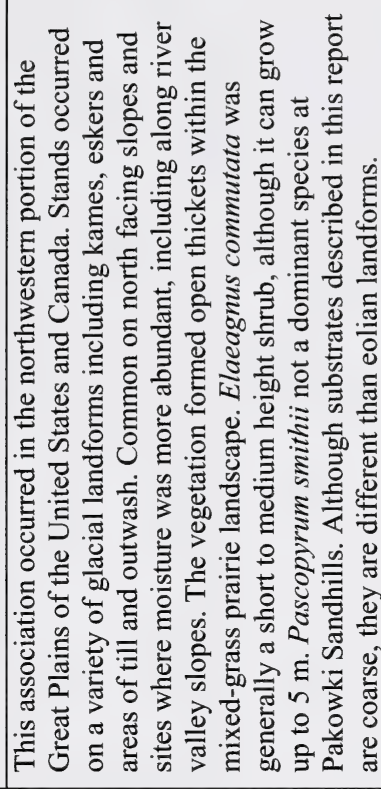 & 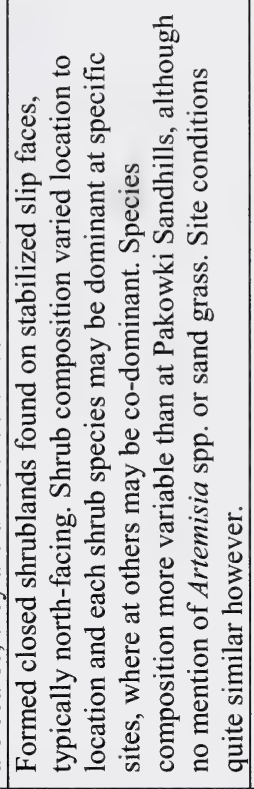 & 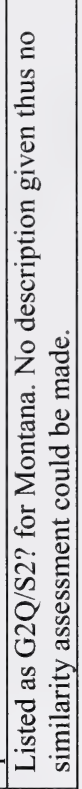 \\
\hline 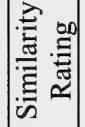 & N & $m$ & N & a. \\
\hline 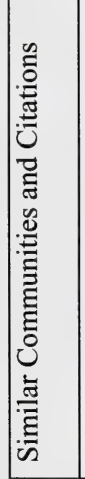 & 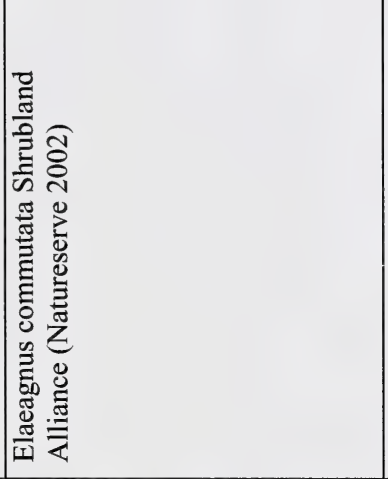 & 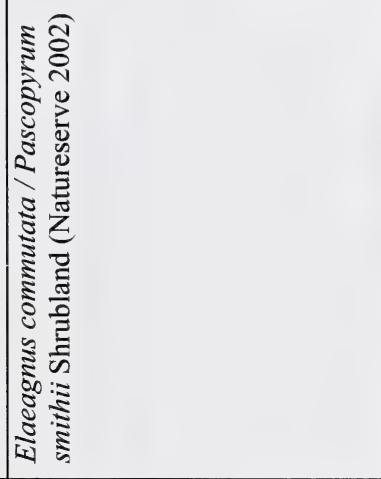 & 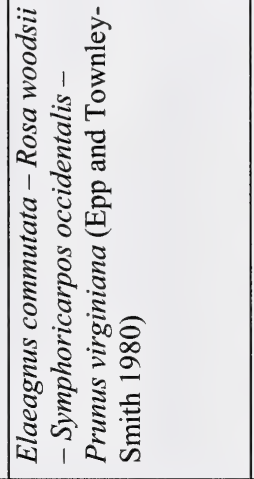 & 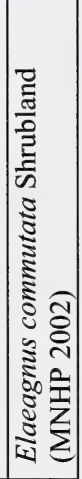 \\
\hline 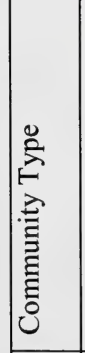 & 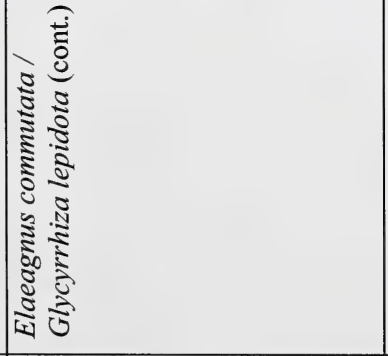 & & 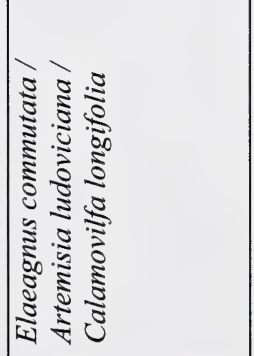 & \\
\hline $\begin{array}{l}\frac{\mathscr{E}}{0} \\
\frac{\pi}{U}\end{array}$ & 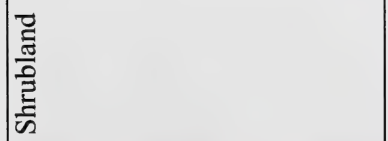 & & 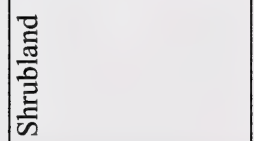 & \\
\hline
\end{tabular}




\begin{tabular}{|c|c|c|c|c|}
\hline 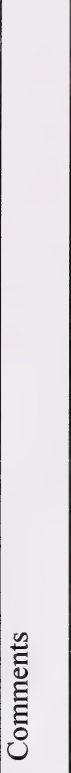 & 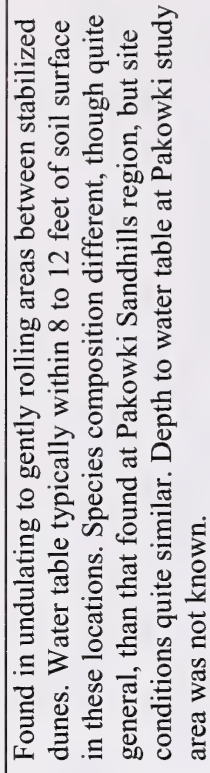 & 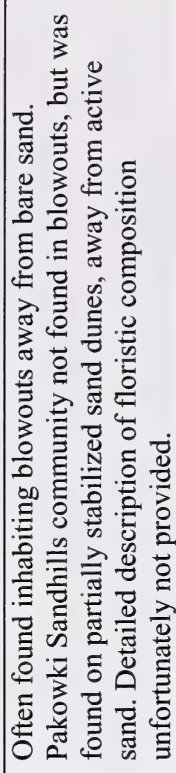 & 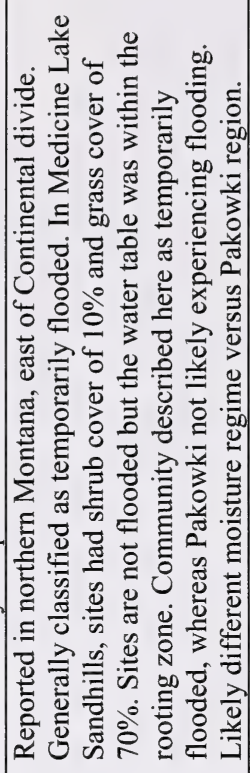 & 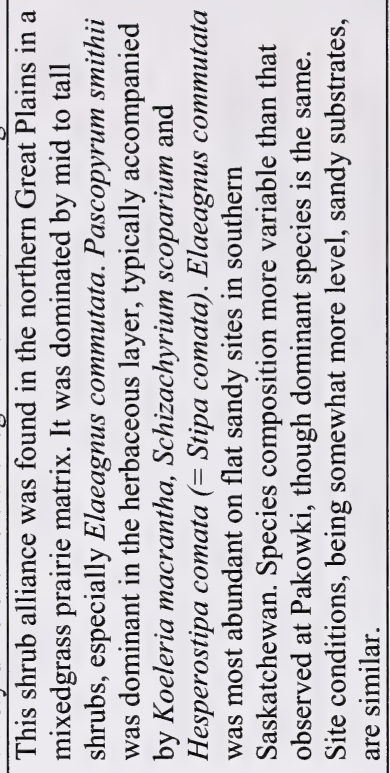 \\
\hline 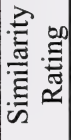 & v & N & N & $N$ \\
\hline 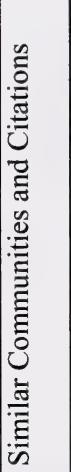 & 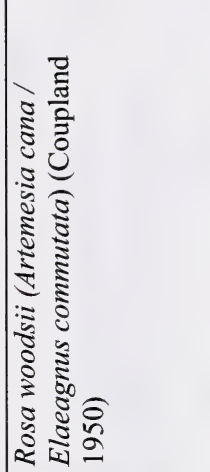 & 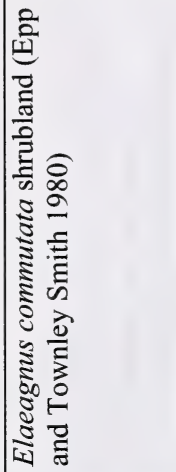 & 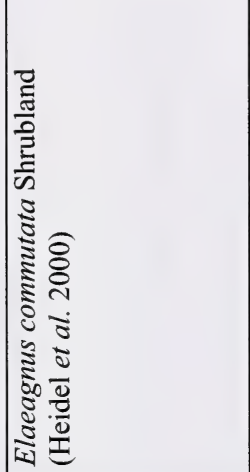 & 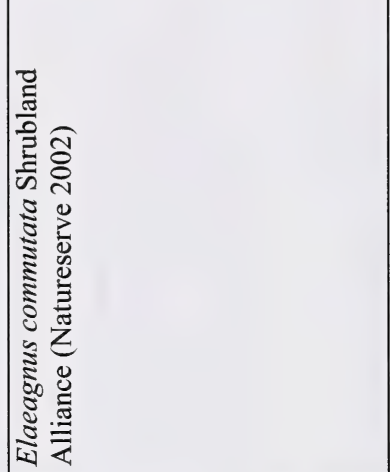 \\
\hline 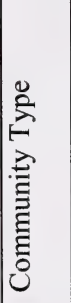 & 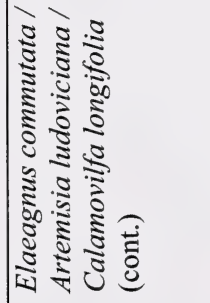 & & & \\
\hline $\begin{array}{l}\frac{v}{0} \\
\frac{\tilde{\sigma}}{U}\end{array}$ & 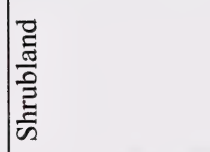 & & & \\
\hline
\end{tabular}




\begin{tabular}{|c|c|c|c|c|}
\hline 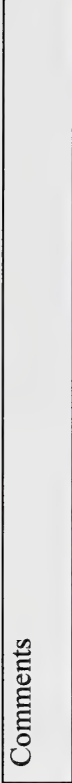 & 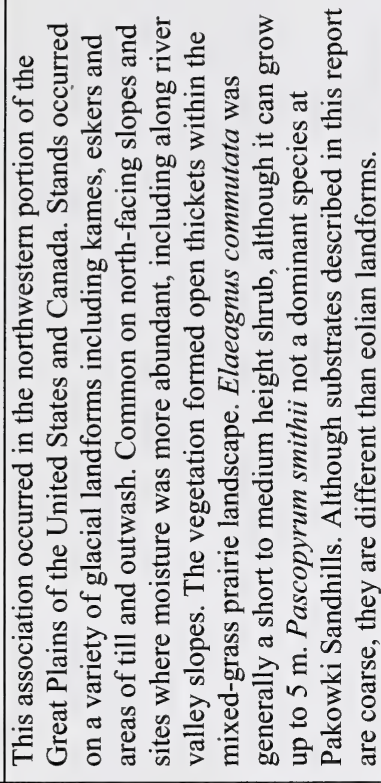 & 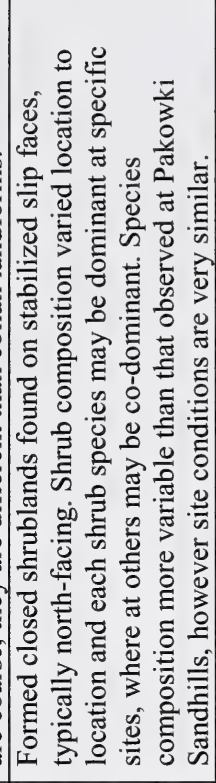 & 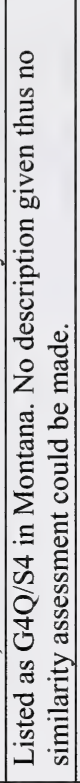 & 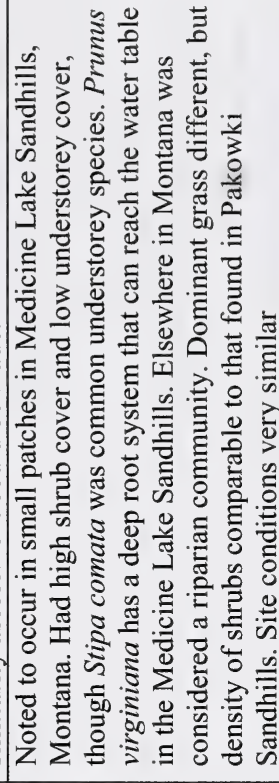 \\
\hline 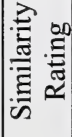 & $m$ & $N$ & $\approx$ & $N$ \\
\hline 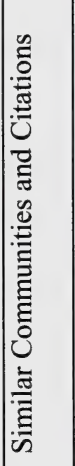 & 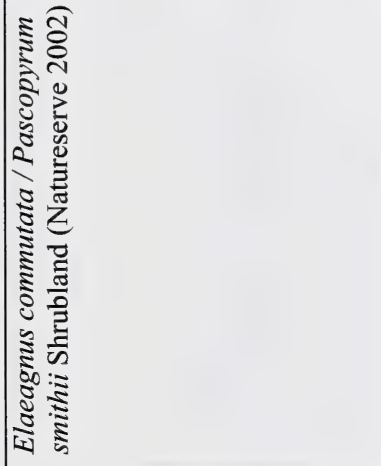 & 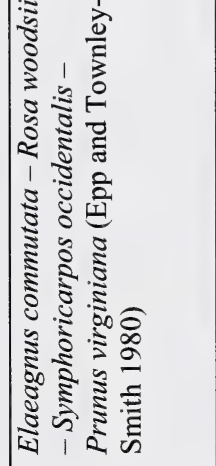 & 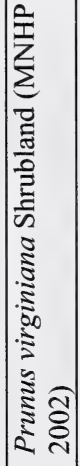 & 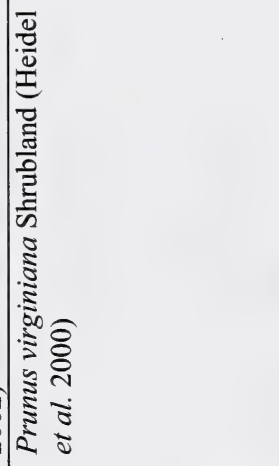 \\
\hline 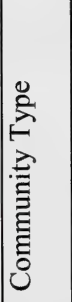 & 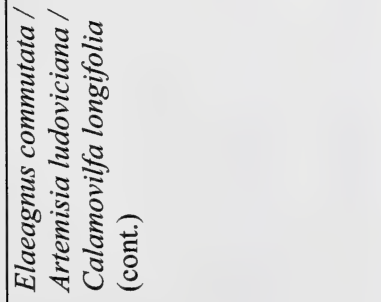 & 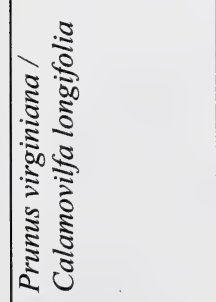 & & \\
\hline 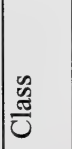 & 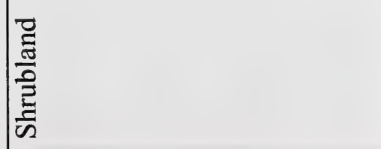 & 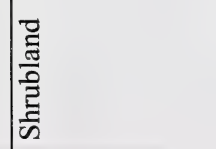 & & \\
\hline
\end{tabular}

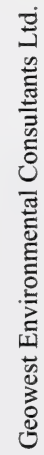




\begin{tabular}{|c|c|c|c|c|}
\hline 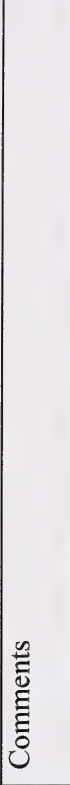 & 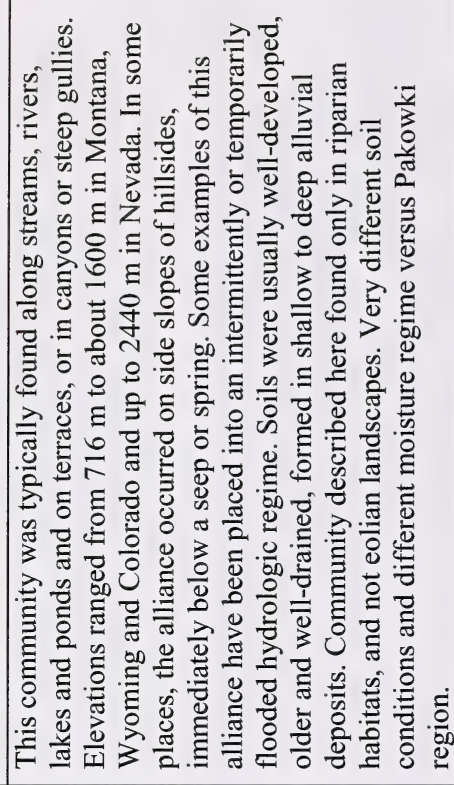 & 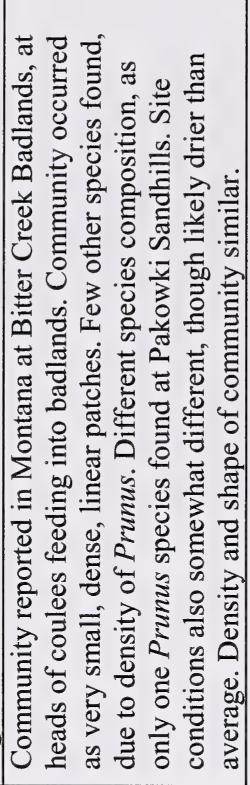 & 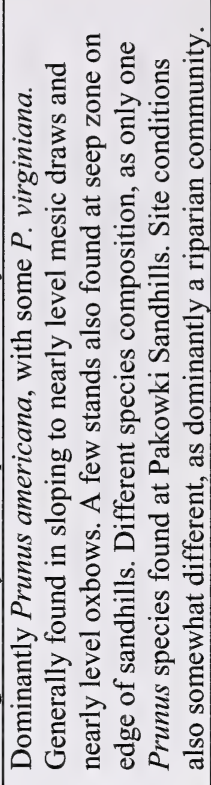 & 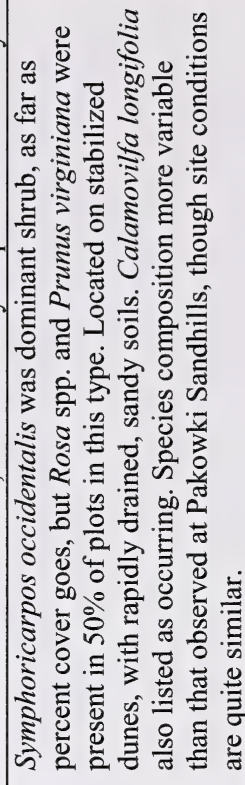 \\
\hline 寄 & $m$ & $m$ & $m$ & $m$ \\
\hline 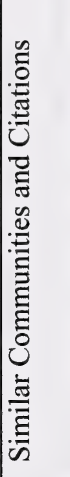 & 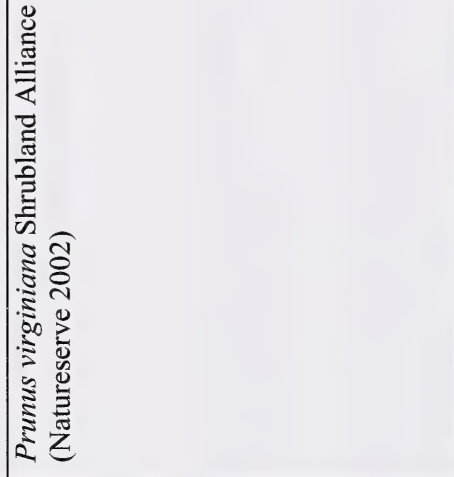 & 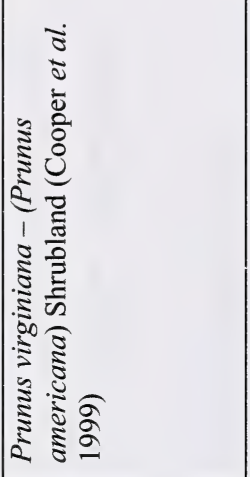 & 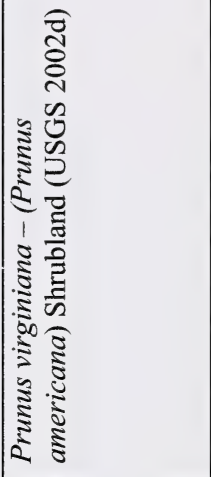 & 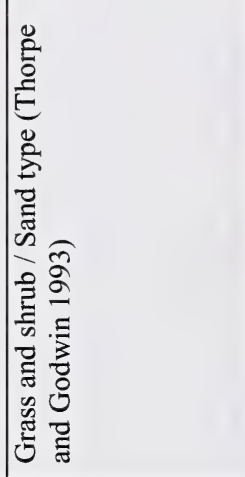 \\
\hline 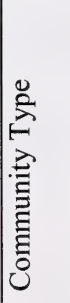 & 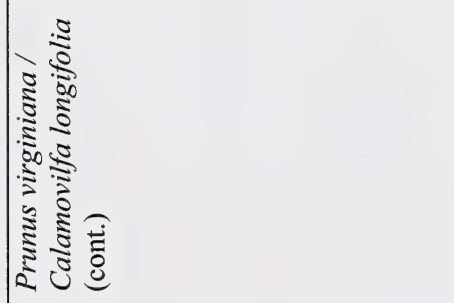 & & & \\
\hline 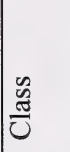 & 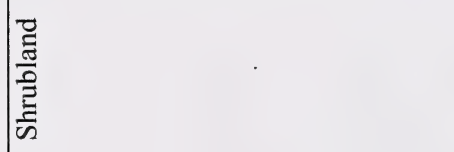 & & & \\
\hline
\end{tabular}




\begin{tabular}{|c|c|c|c|c|c|c|}
\hline 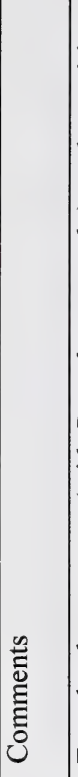 & 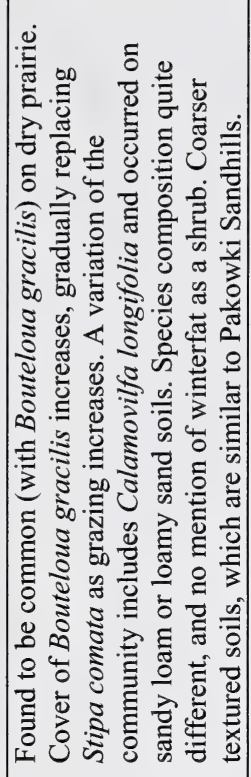 & 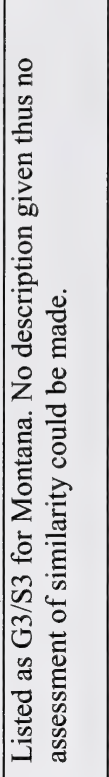 & 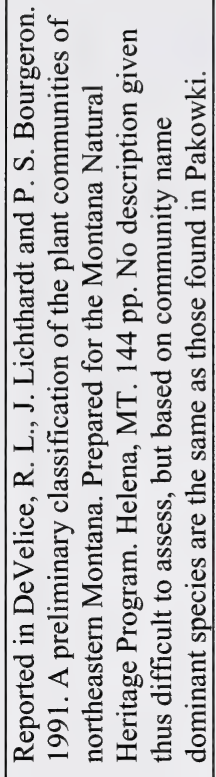 & 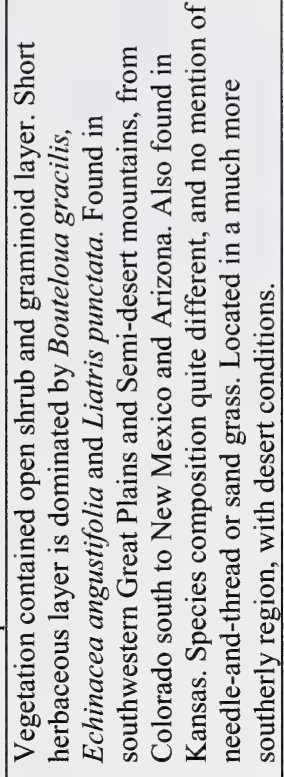 & 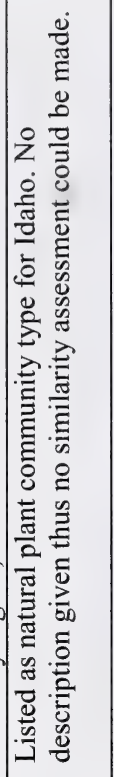 & 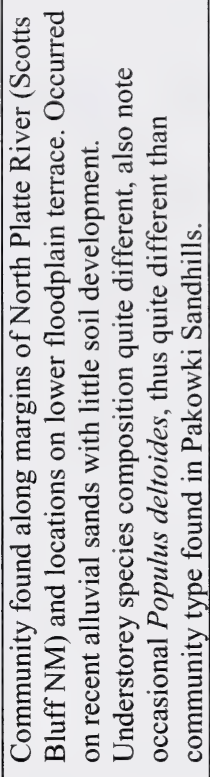 \\
\hline 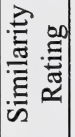 & m & a. & $\sim$ & $m$ & a. & $m$ \\
\hline 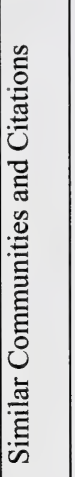 & 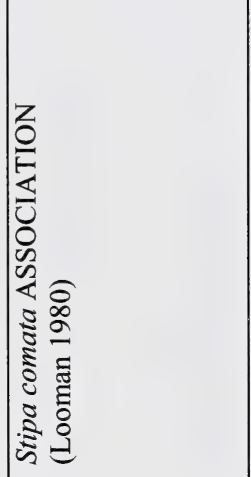 & 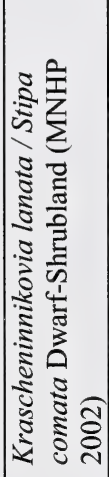 & 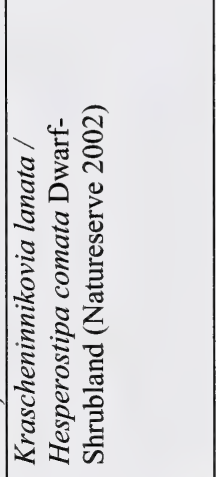 & 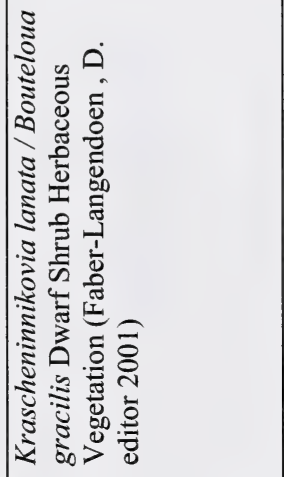 & 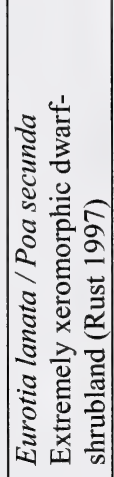 & 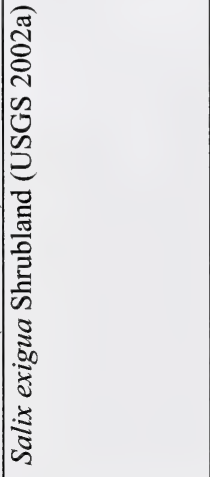 \\
\hline 离 & 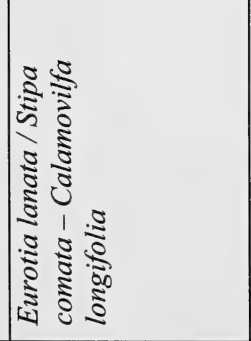 & & & & & 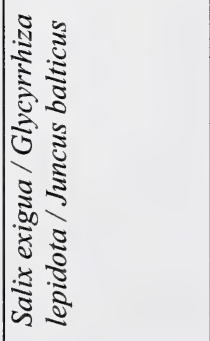 \\
\hline $\begin{array}{l}\frac{w}{\sigma} \\
\frac{\sigma}{0}\end{array}$ & 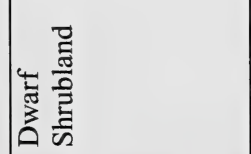 & & & & & 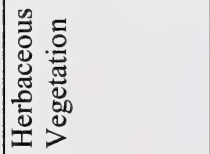 \\
\hline
\end{tabular}




\begin{tabular}{|c|c|c|c|c|}
\hline 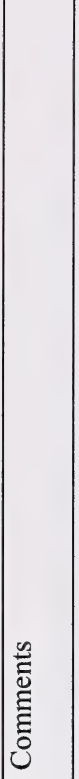 & 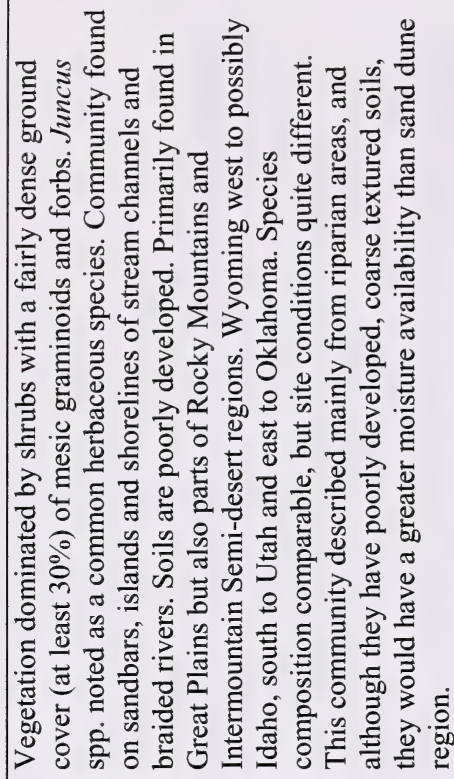 & 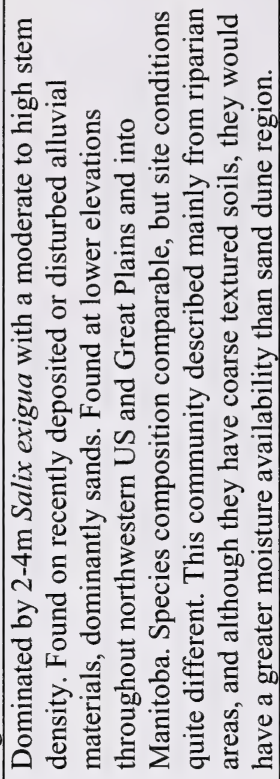 & 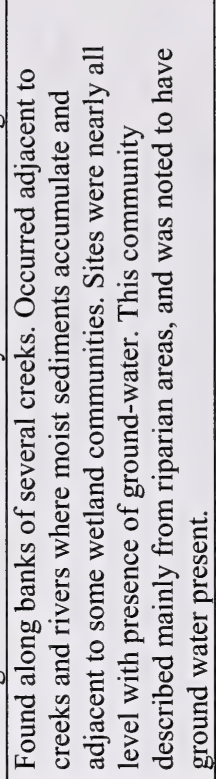 & 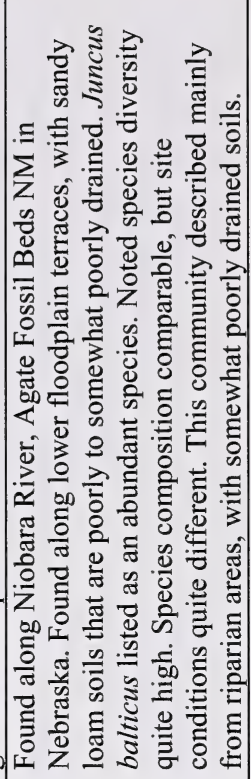 \\
\hline 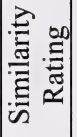 & $m$ & $m$ & $m$ & $N$ \\
\hline 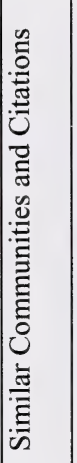 & 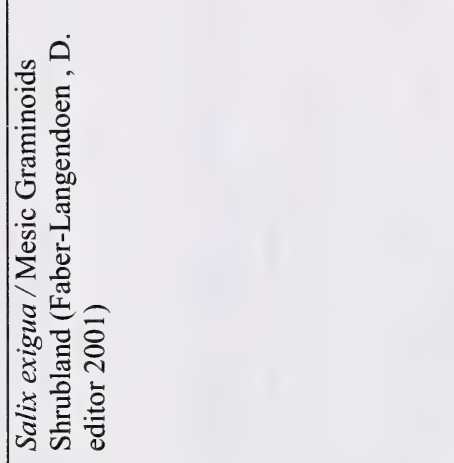 & 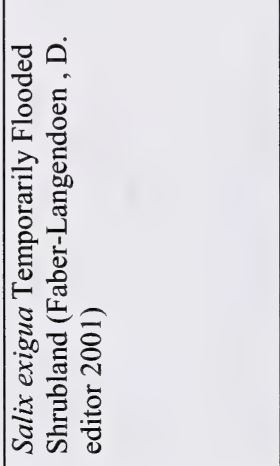 & 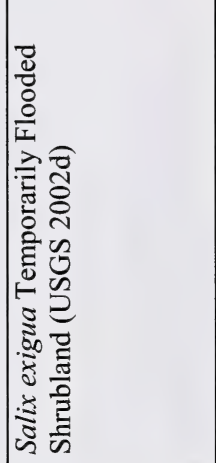 & 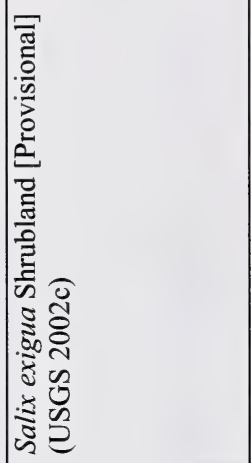 \\
\hline 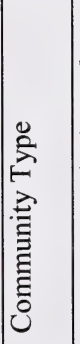 & 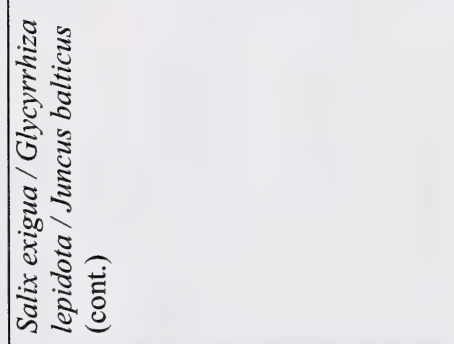 & & & \\
\hline 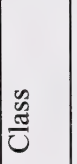 & 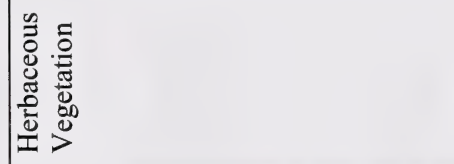 & & & \\
\hline
\end{tabular}




\begin{tabular}{|c|c|c|c|c|c|c|c|c|c|}
\hline 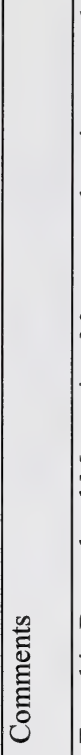 & 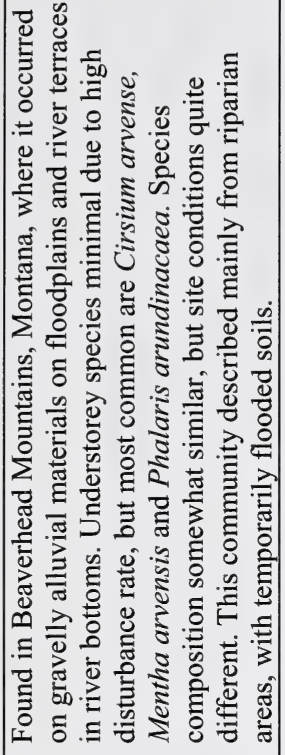 & 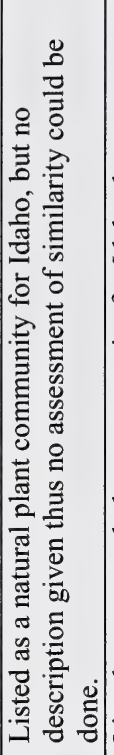 & 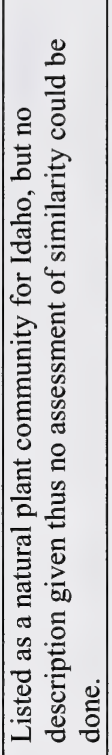 & 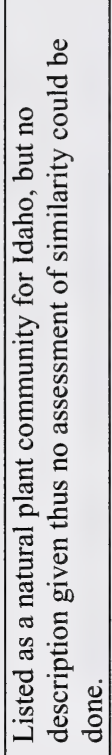 & 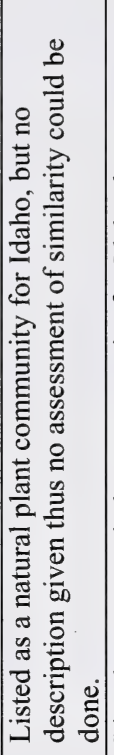 & 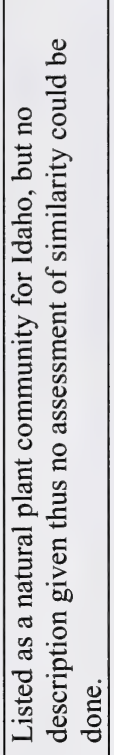 & 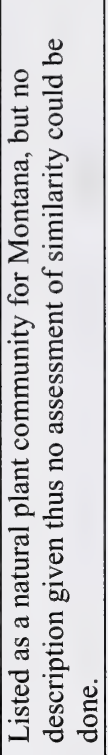 & 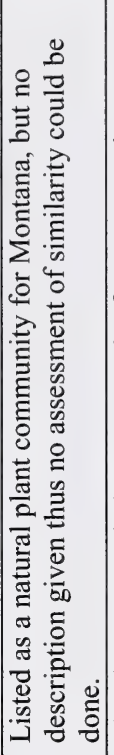 & 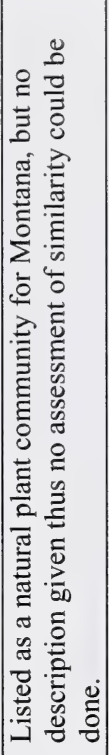 \\
\hline 窝 & $m$ & a. & a. & a. & a. & a. & a. & a. & r. \\
\hline 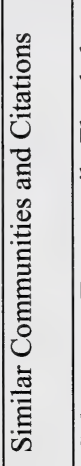 & 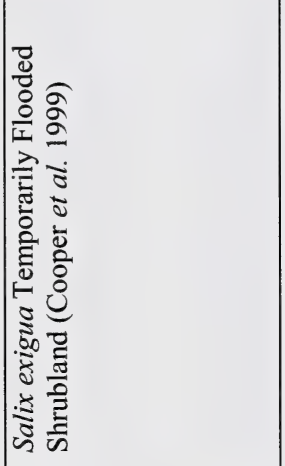 & 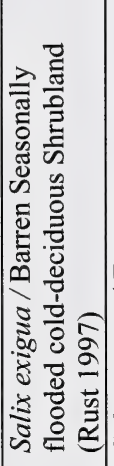 & 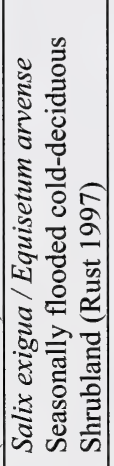 & 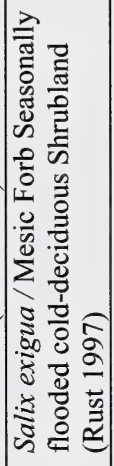 & 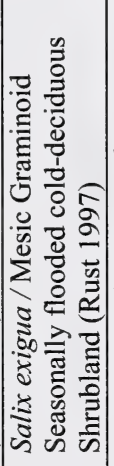 & 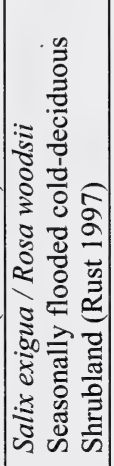 & 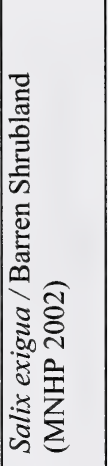 & 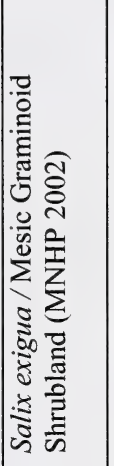 & 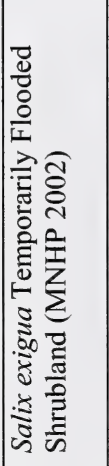 \\
\hline 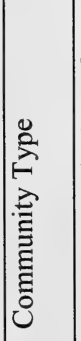 & 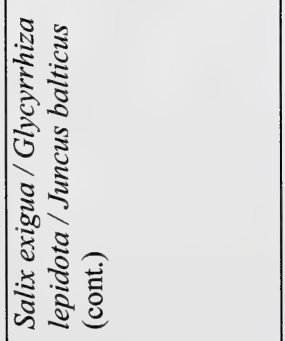 & & & & & & & & \\
\hline $\begin{array}{l}\frac{w}{U} \\
\frac{\tilde{U}}{U}\end{array}$ & 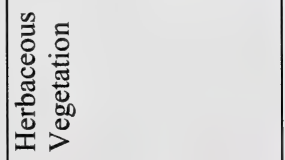 & & & & & & & & \\
\hline
\end{tabular}




\begin{tabular}{|c|c|c|c|c|c|c|c|c|c|}
\hline 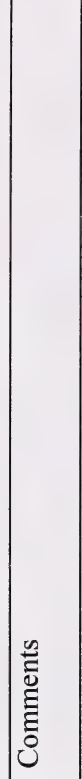 & 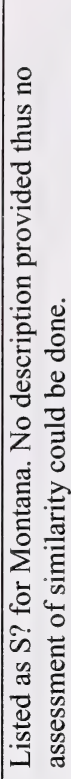 & 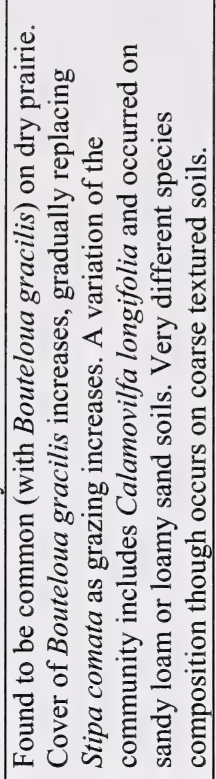 & 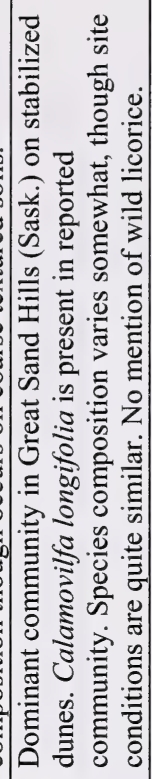 & 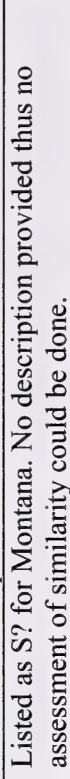 & 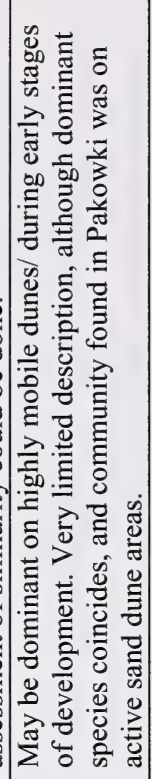 & 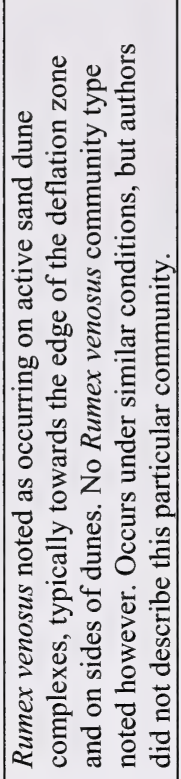 & 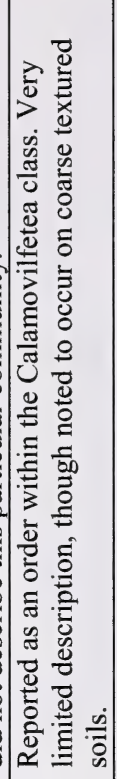 & 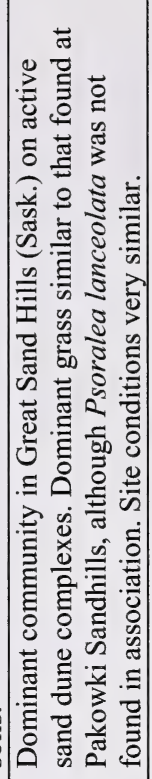 & 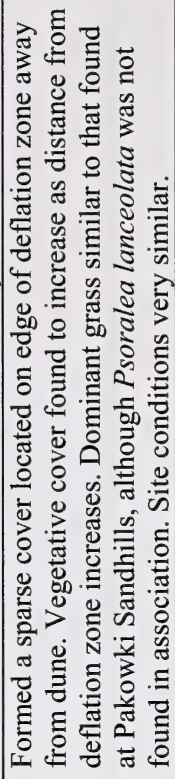 \\
\hline 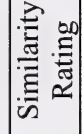 & & $m$ & $N$ & a. & - & $m$ & $m$ & $N$ & $N$ \\
\hline 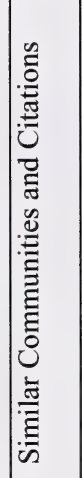 & 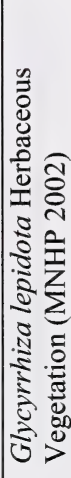 & 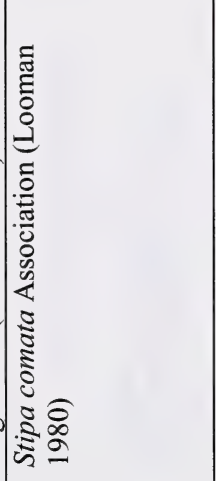 & 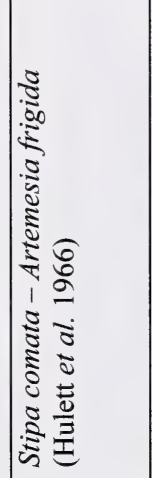 & 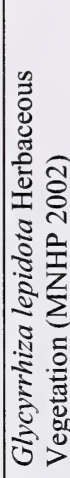 & 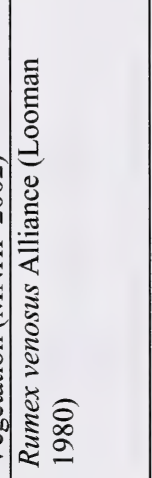 & 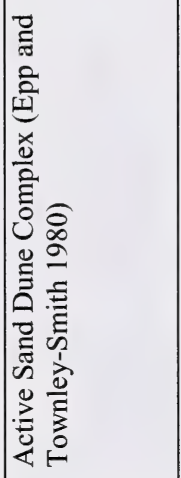 & 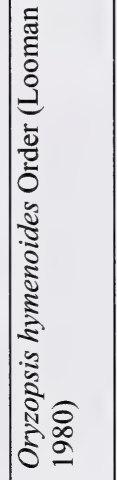 & 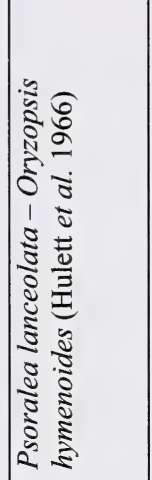 & 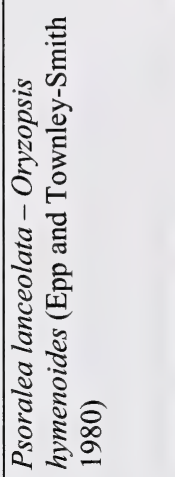 \\
\hline 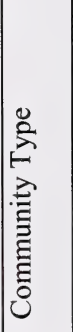 & 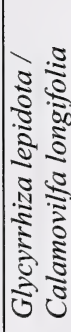 & 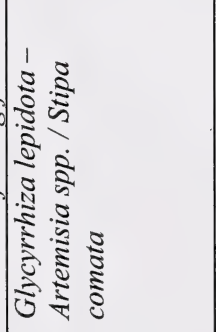 & & & 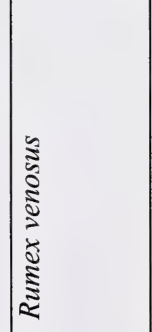 & & 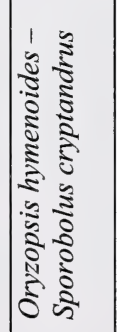 & & \\
\hline $\begin{array}{l}\frac{\tilde{y}}{U} \\
\frac{\sigma}{U}\end{array}$ & 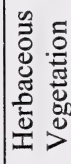 & 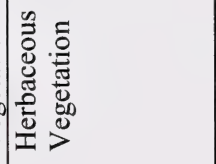 & & & 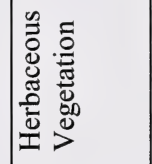 & & 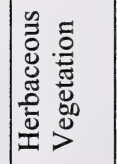 & & \\
\hline
\end{tabular}




\begin{tabular}{|c|c|c|c|c|c|c|c|}
\hline 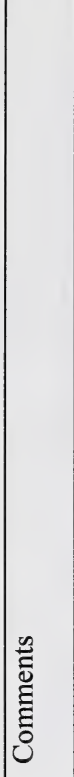 & 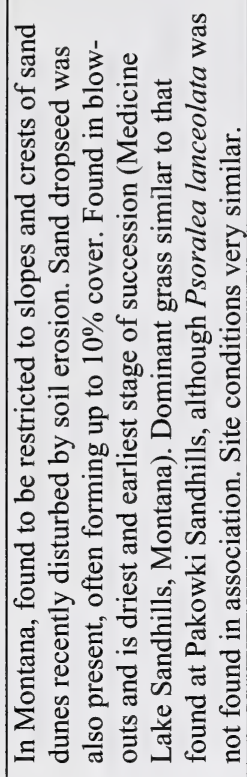 & 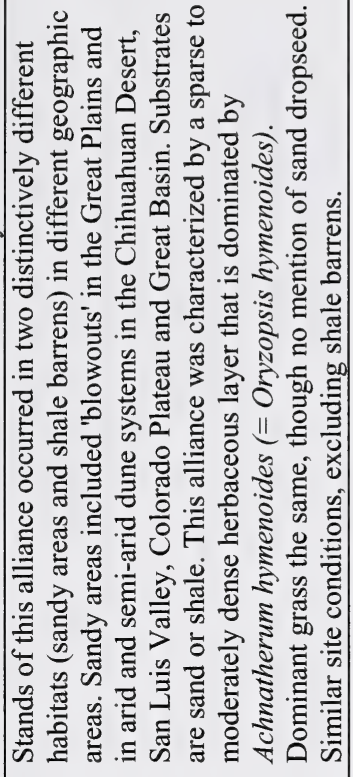 & 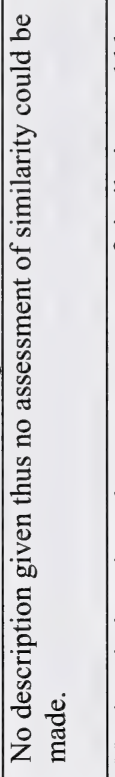 & 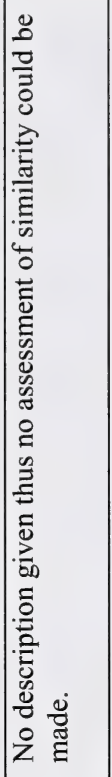 & 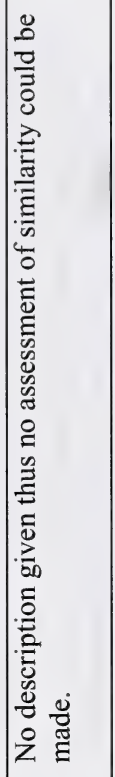 & 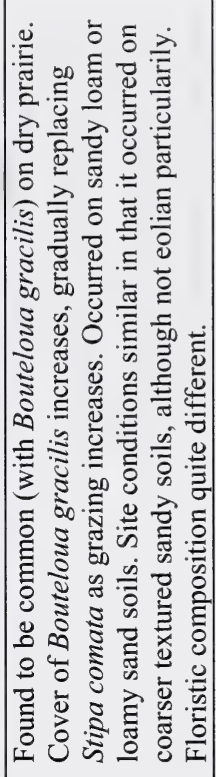 & 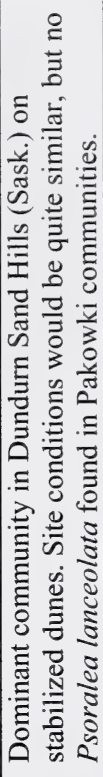 \\
\hline 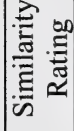 & $v$ & $\sim$ & $\curvearrowright$. & ح. & ค. & $m$ & $m$ \\
\hline 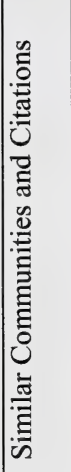 & 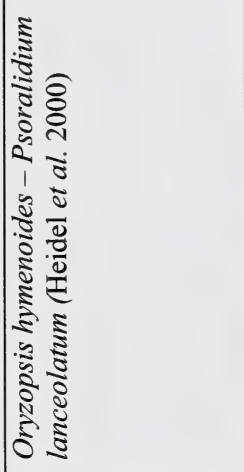 & 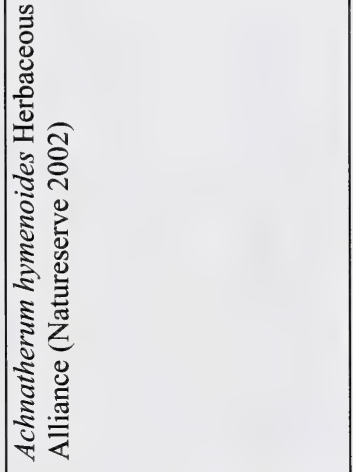 & 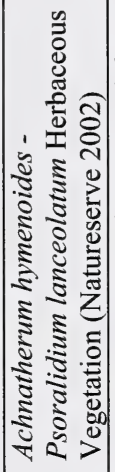 & 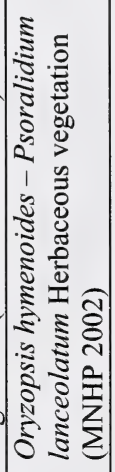 & 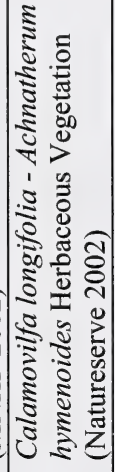 & 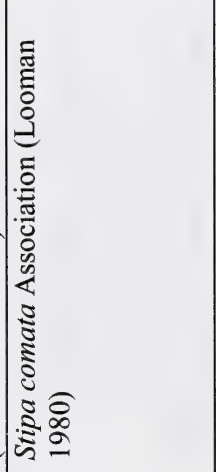 & 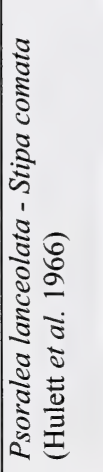 \\
\hline 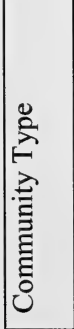 & 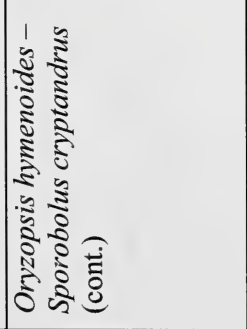 & & & & & 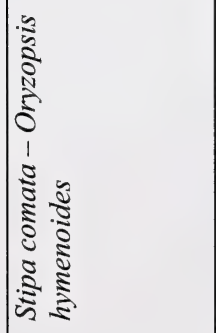 & \\
\hline $\begin{array}{l}\frac{\tilde{U}}{0} \\
\frac{\tilde{\sigma}}{0}\end{array}$ & 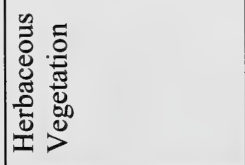 & & & & & 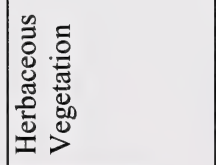 & \\
\hline
\end{tabular}




\begin{tabular}{|c|c|c|c|c|}
\hline 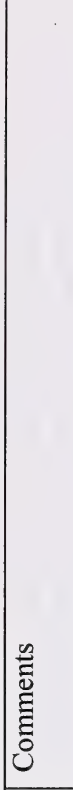 & 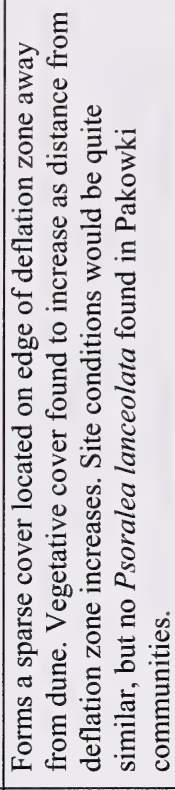 & 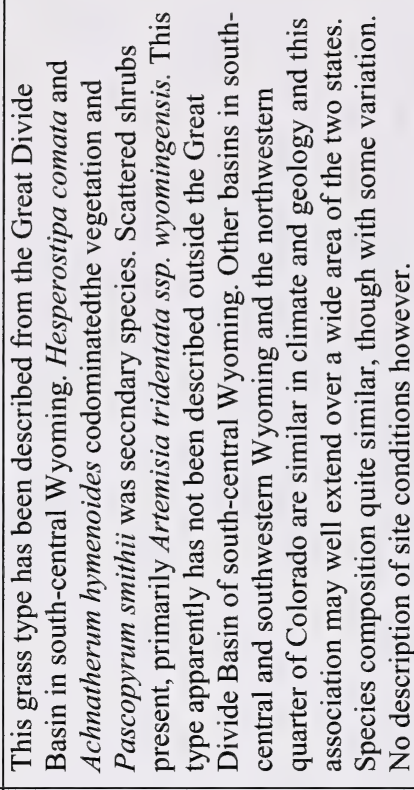 & 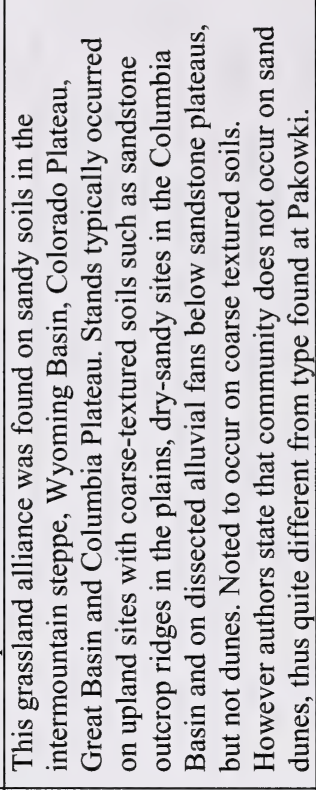 & 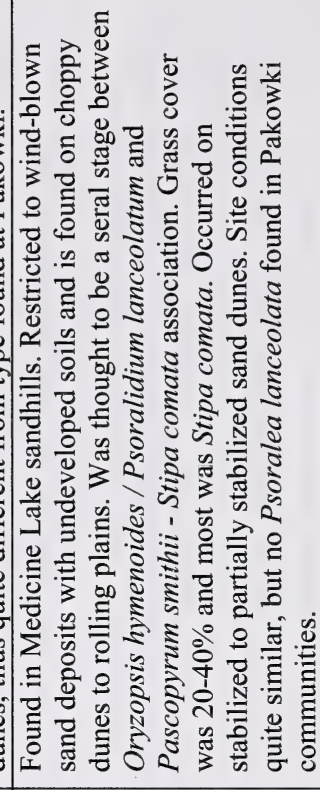 \\
\hline 㯊 & & N & $m$ & $\sim$ \\
\hline 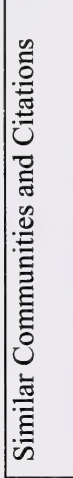 & 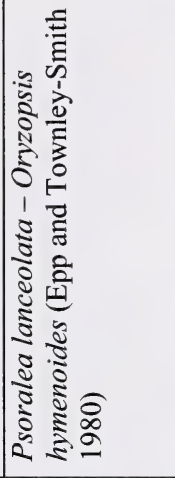 & 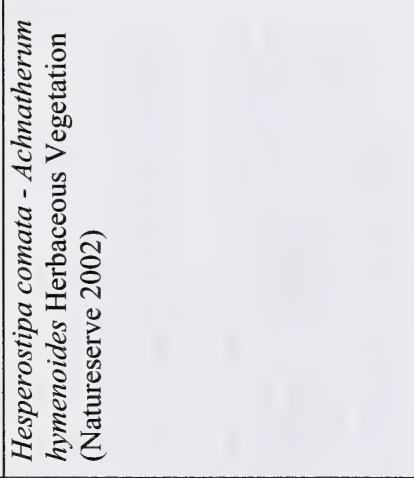 & 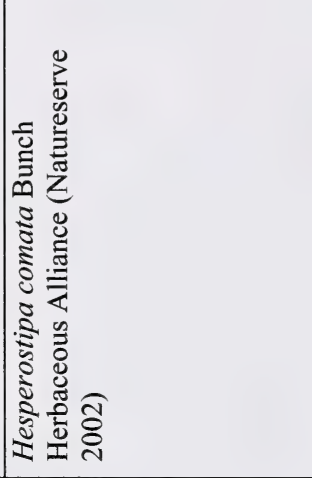 & 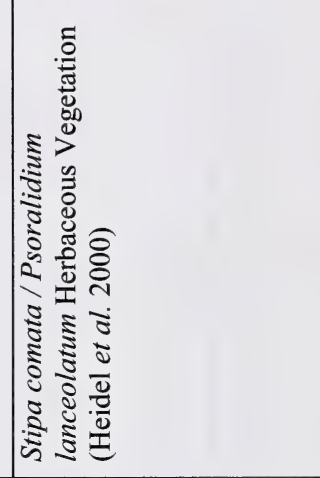 \\
\hline 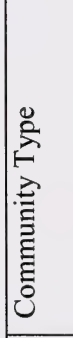 & 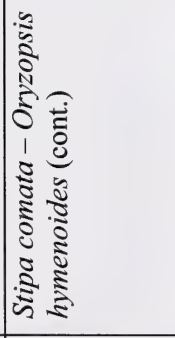 & & & \\
\hline & 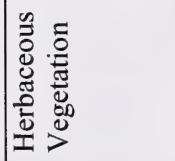 & & & \\
\hline
\end{tabular}




\begin{tabular}{|c|c|c|c|c|c|c|c|}
\hline & 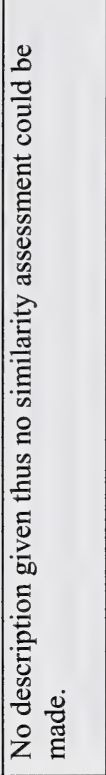 & 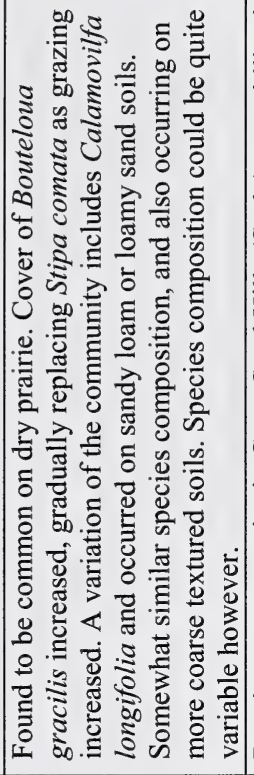 & 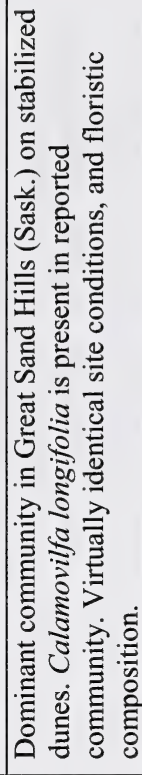 & 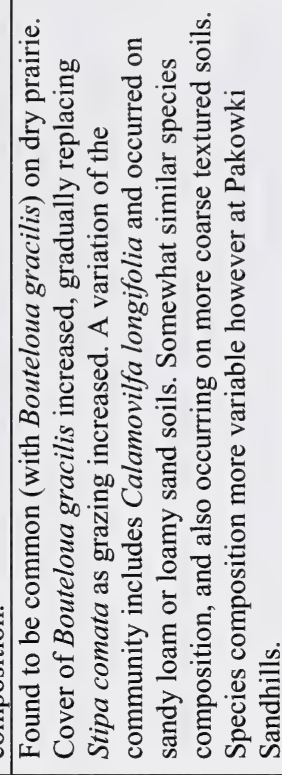 & 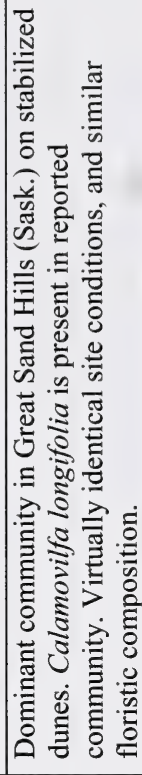 & 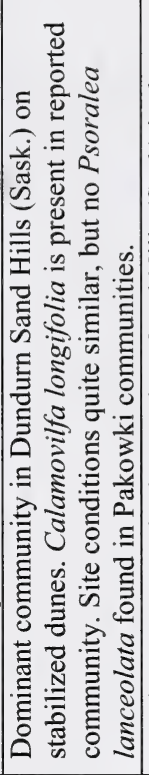 & 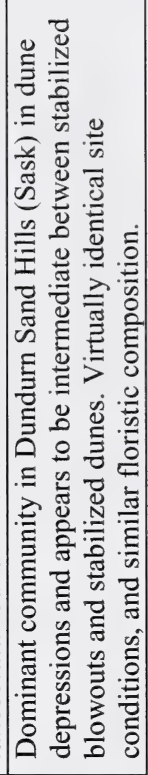 \\
\hline 部 & & $m$ & - & $\sim$ & $\sim$ & $m$ & - \\
\hline 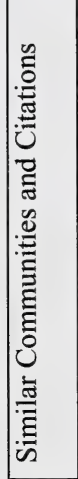 & 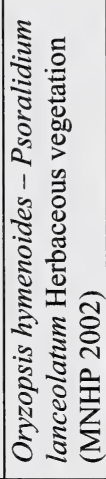 & 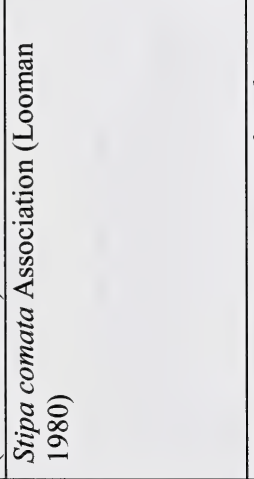 & 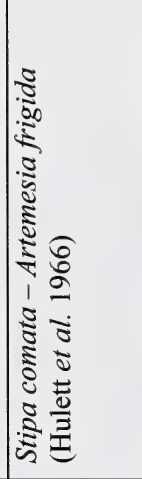 & 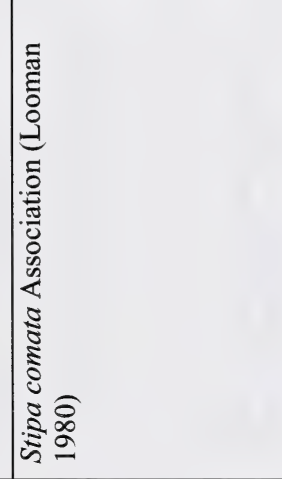 & 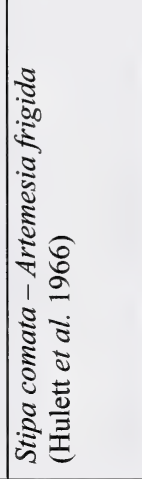 & 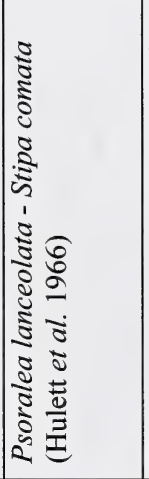 & 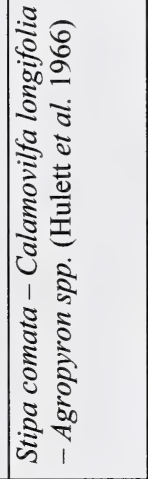 \\
\hline 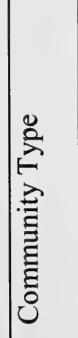 & 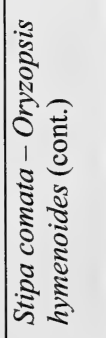 & 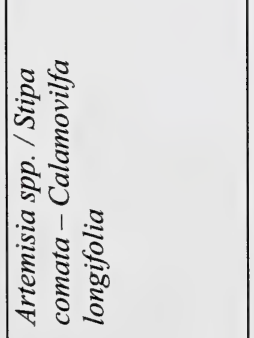 & & 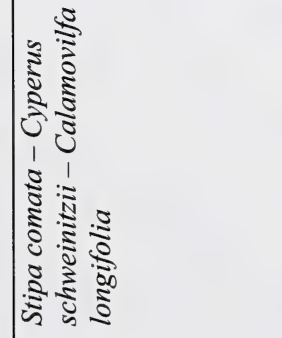 & & & \\
\hline $\begin{array}{l}\text { 离 } \\
\text { dut }\end{array}$ & 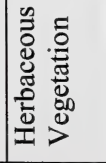 & 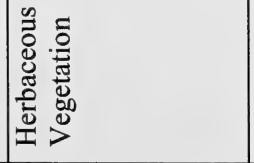 & 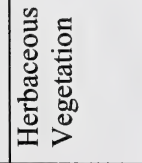 & 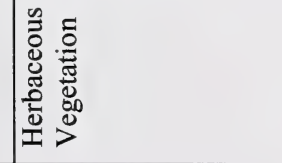 & & & \\
\hline
\end{tabular}




\begin{tabular}{|c|c|c|c|c|}
\hline & 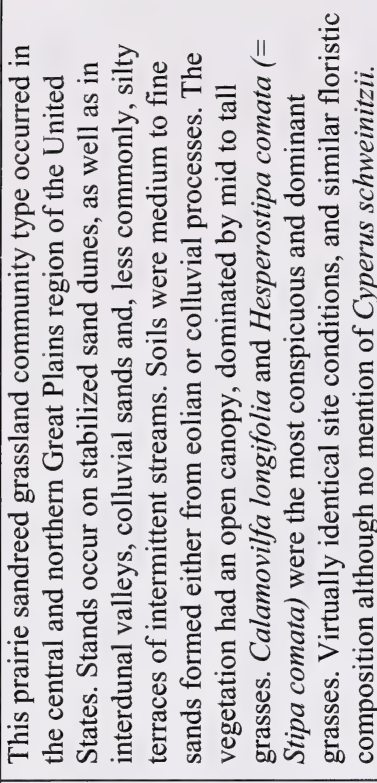 & 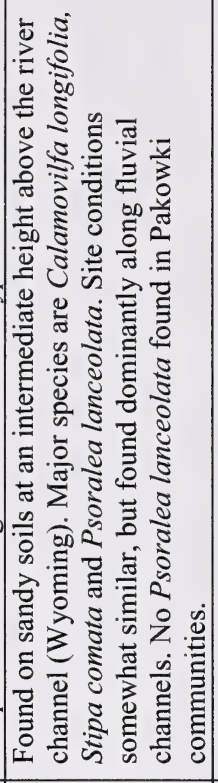 & 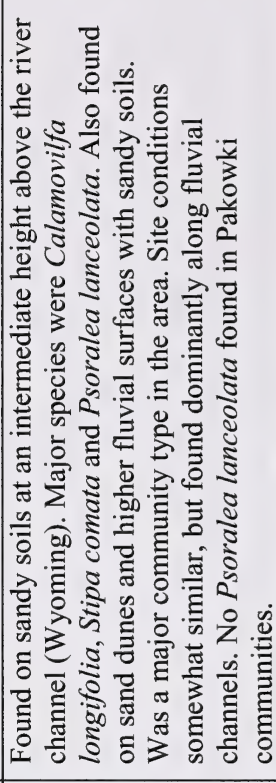 & 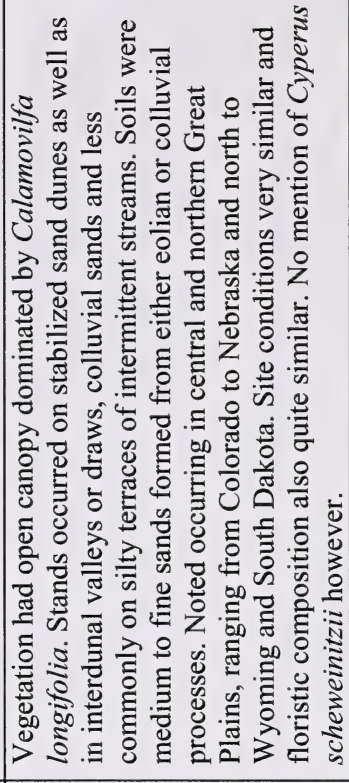 \\
\hline 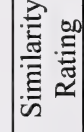 & & $m$ & $m$ & $\sim$ \\
\hline 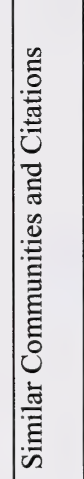 & 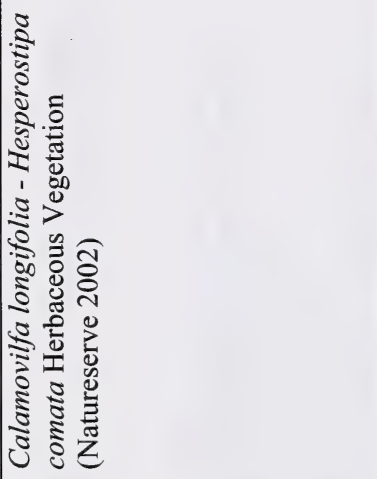 & 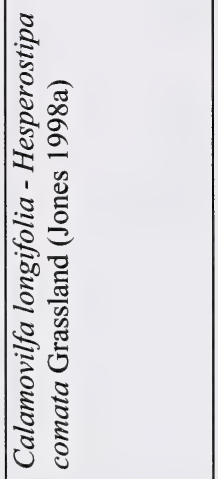 & 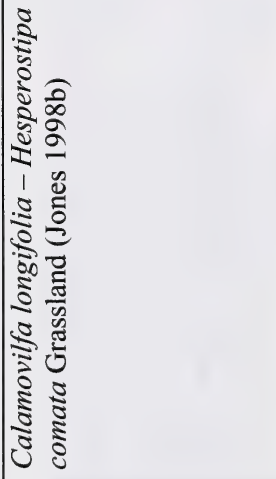 & 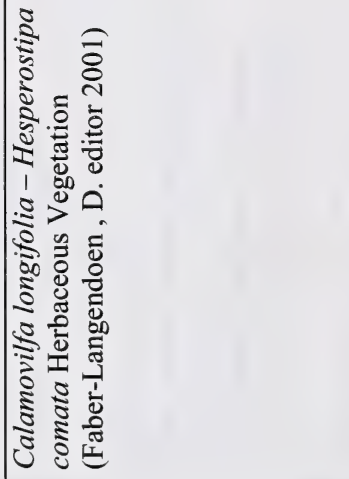 \\
\hline 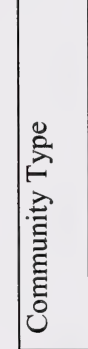 & 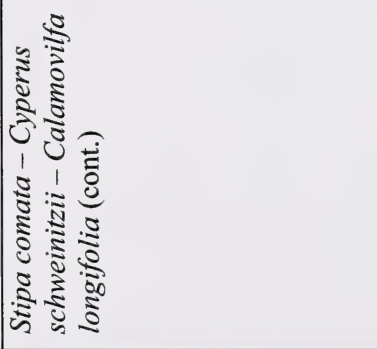 & & & \\
\hline 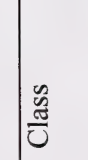 & 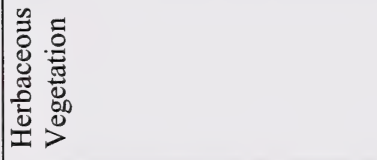 & & & \\
\hline
\end{tabular}




\begin{tabular}{|c|c|c|c|c|c|c|c|}
\hline 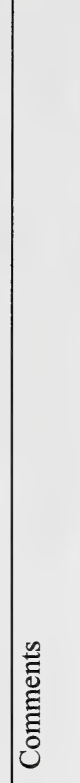 & 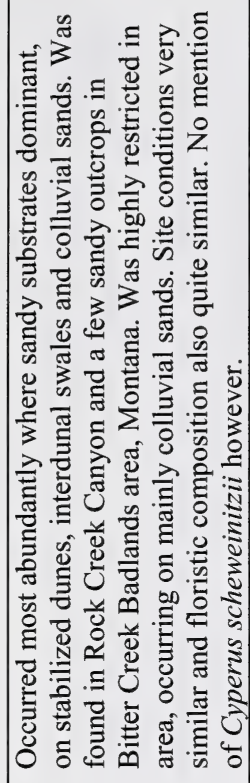 & 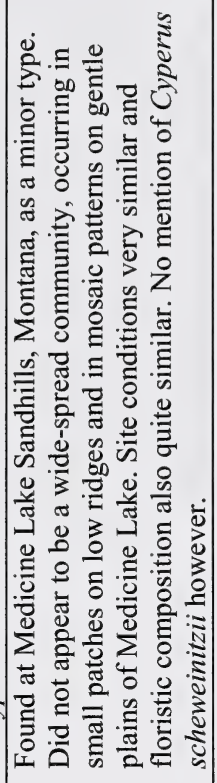 & 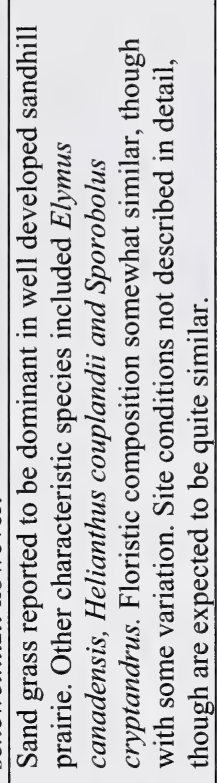 & 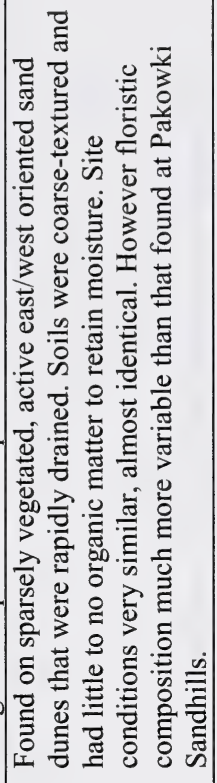 & 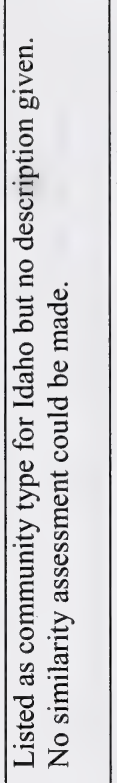 & 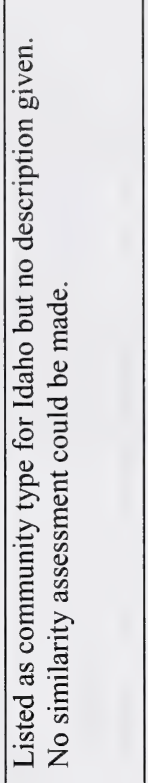 & 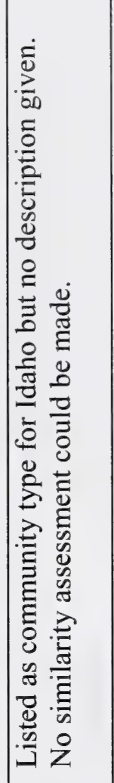 \\
\hline 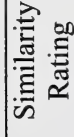 & I & N & N & $m$ & a. & a. & a. \\
\hline 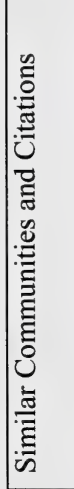 & 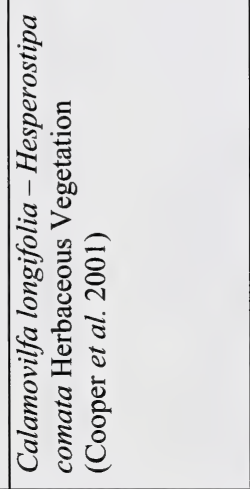 & 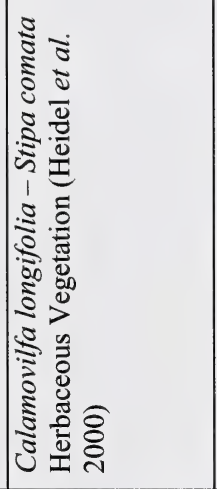 & 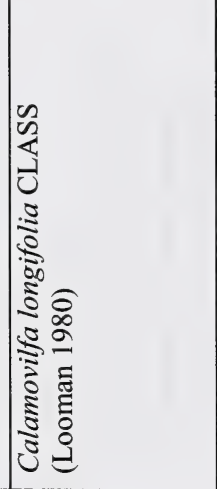 & 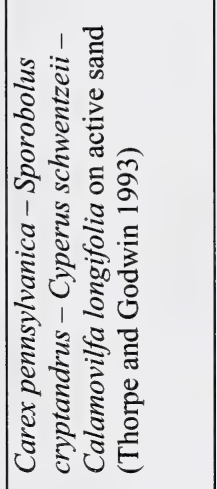 & 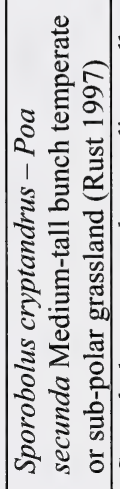 & 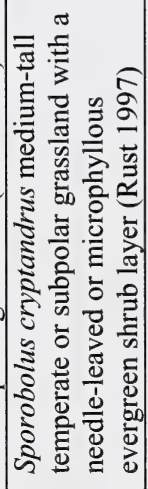 & 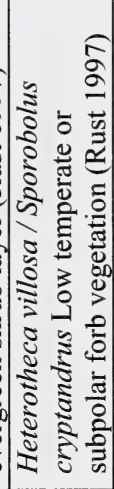 \\
\hline 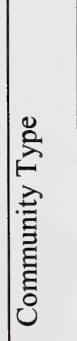 & 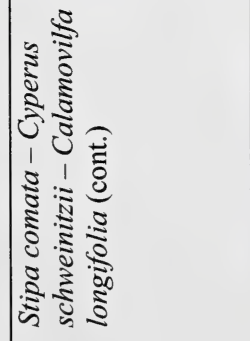 & & 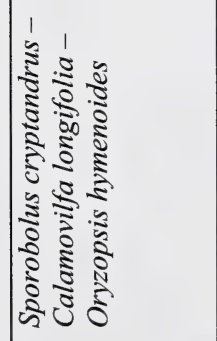 & & & & \\
\hline $\begin{array}{l}\frac{\tilde{a}}{0} \\
\frac{\sigma}{U}\end{array}$ & 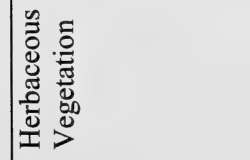 & & 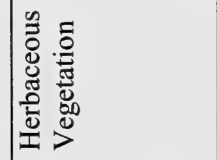 & & & & \\
\hline
\end{tabular}




\begin{tabular}{|c|c|c|c|c|}
\hline $\begin{array}{l}\stackrel{0}{\Xi} \\
\stackrel{\Xi}{\Xi} \\
0 \\
0\end{array}$ & 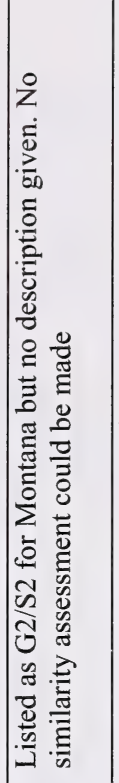 & 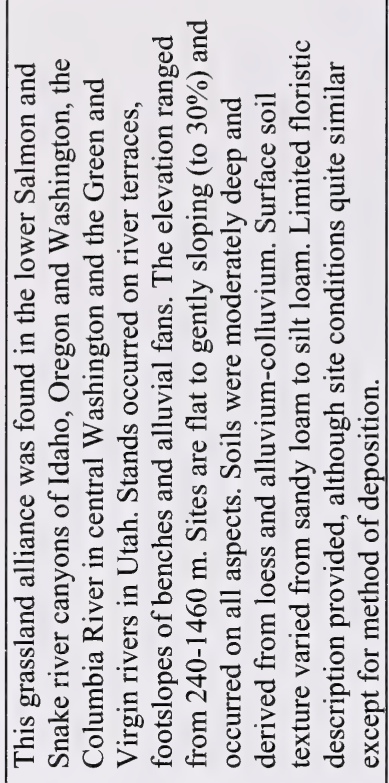 & 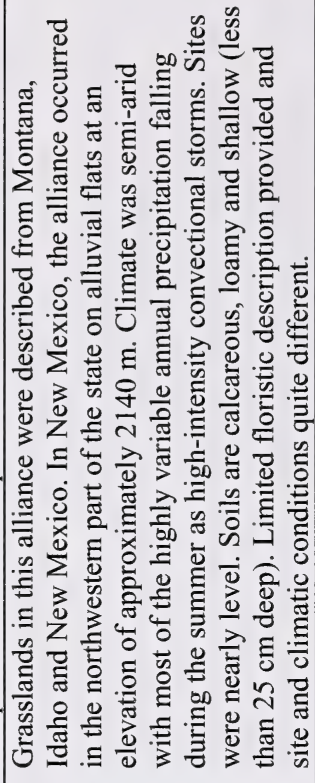 & 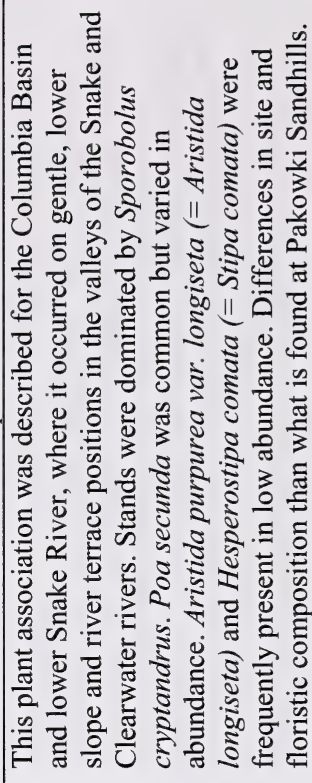 \\
\hline 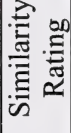 & $\sigma$ & $m$ & $m$ & $m$ \\
\hline 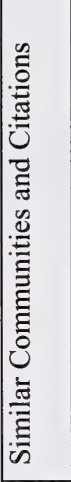 & 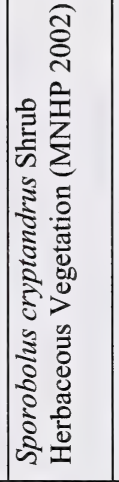 & 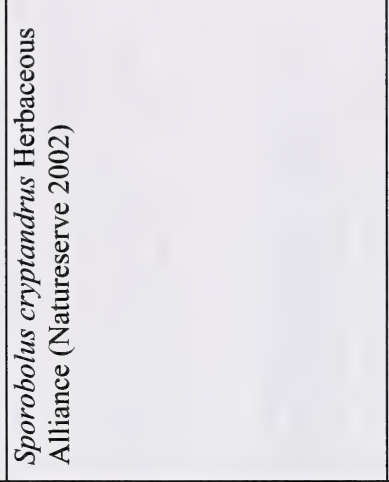 & 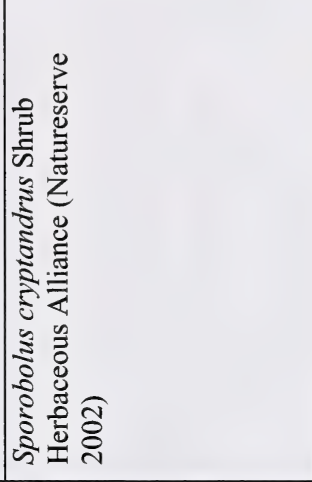 & 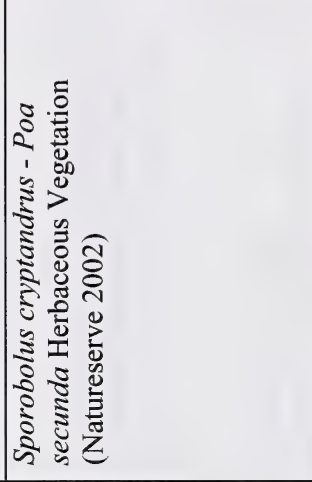 \\
\hline 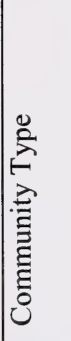 & 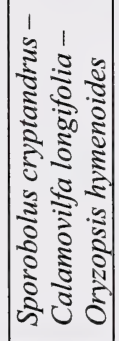 & & & 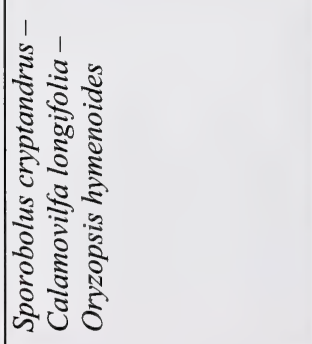 \\
\hline $\begin{array}{l}\text { ov } \\
\frac{\tilde{\sigma}}{0}\end{array}$ & 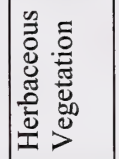 & & & 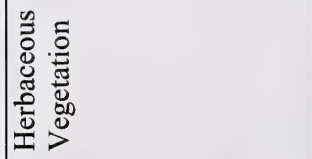 \\
\hline
\end{tabular}




\begin{tabular}{|c|c|c|c|c|}
\hline & 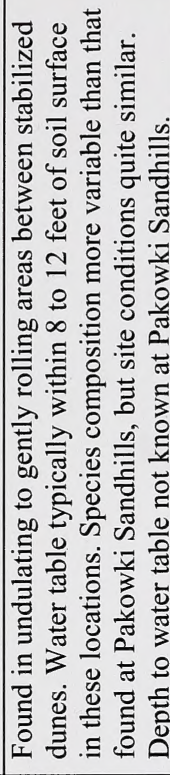 & 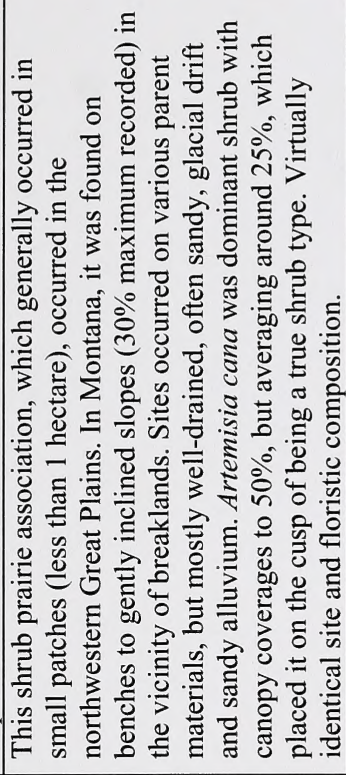 & 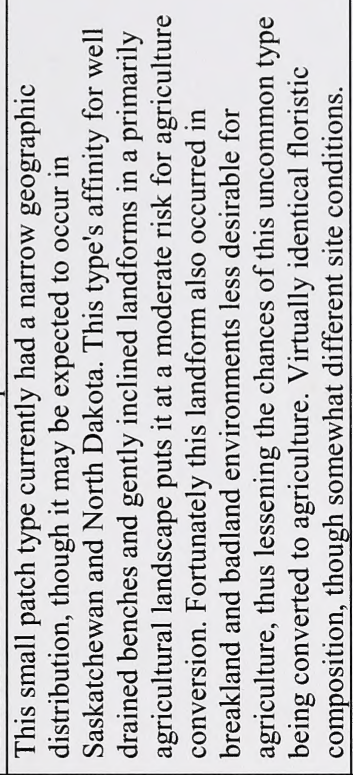 & 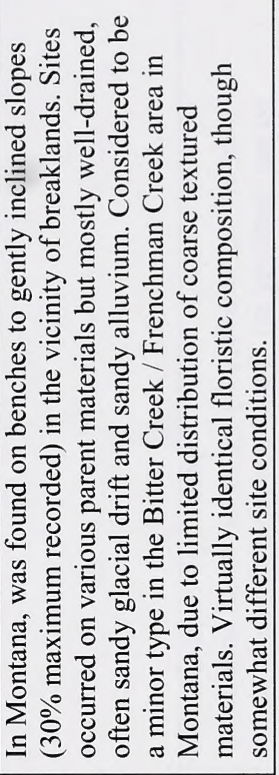 \\
\hline 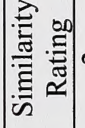 & & - & - & - \\
\hline 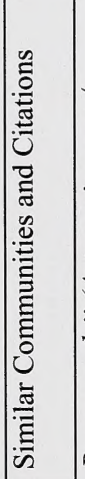 & 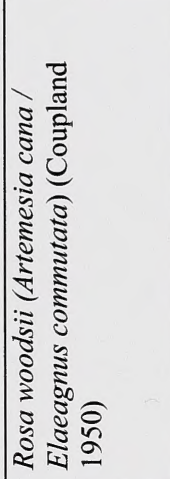 & 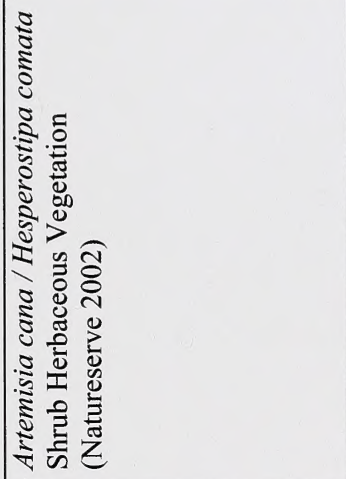 & 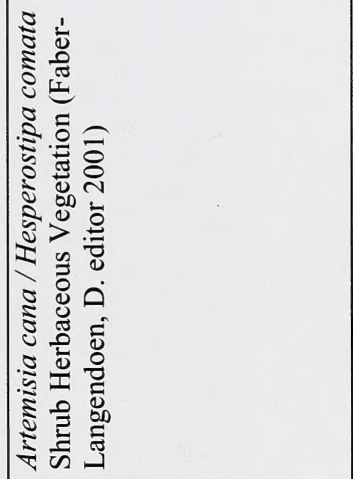 & 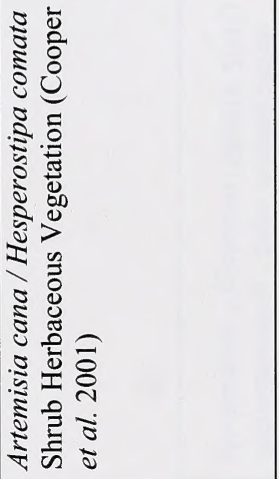 \\
\hline 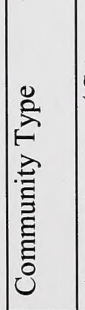 & 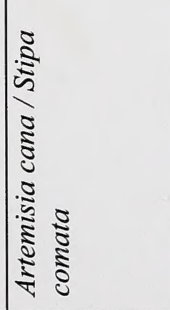 & & & \\
\hline $\begin{array}{l}\frac{b}{0} \\
\frac{\pi}{U}\end{array}$ & 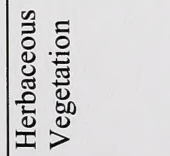 & & & \\
\hline
\end{tabular}




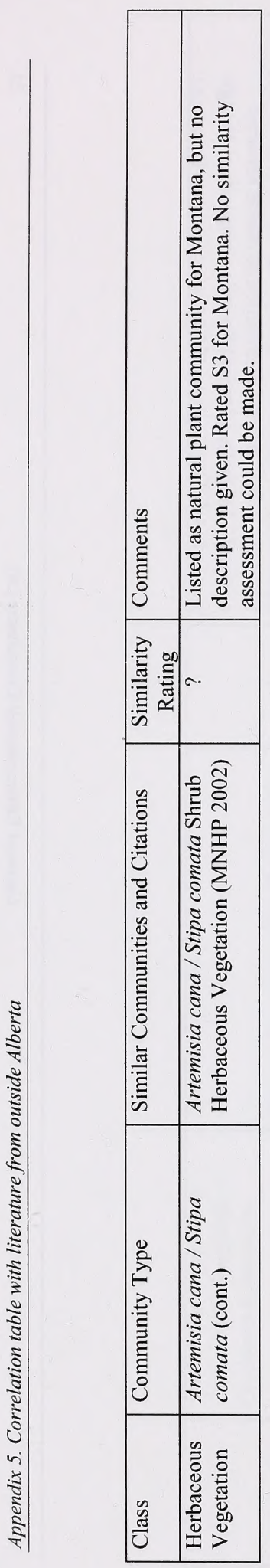


National Library of Canada
Bibliotheque nationale du Canada

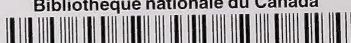

33286527363804 